\title{
Topologias enumeravelmente compactas em grupos abelianos de não torção via ultrafiltros seletivos
}

\author{
Ana Carolina Boero
}

TESE APRESENTADA

$\mathrm{AO}$

INSTITUTO DE MATEMÁTICA E ESTATÍSTICA

DA

UNIVERSIDADE DE SÃO PAULO

PARA

OBTENÇÃO DO TÍTULO DE DOUTOR

EM

CIÊNCIAS

Área de Concentração: Matemática

Orientador: Prof. Dr. Artur Hideyuki Tomita

Durante a elaboração deste trabalho, a autora recebeu apoio financeiro da CAPES e do CNPq.

- São Paulo, 11 de março de 2011 - 



\title{
Topologias enumeravelmente compactas em grupos abelianos de não torção via ultrafiltros seletivos
}

\author{
Este exemplar corresponde à redação final \\ da tese devidamente corrigida e defendida por \\ Ana Carolina Boero e aprovada pela comissão \\ julgadora.
}

São Paulo, 11 de março de 2011.

\section{Banca examinadora:}

Prof. Dr. Artur Hideyuki Tomita (orientador)

IME-USP

Profa. Dra. Ofelia Teresa Alas

IME-USP

Prof. Dr. Plamen Emilov Kochloukov

UNICAMP

Prof. Dr. Jorge Tulio Mujica Ascui

UNICAMP

Profa. Dra. Irene Castro Pereira

UFPA 

Aos meus pais e irmão. 

Agradeço ao meu orientador, Prof. Dr. Artur Hideyuki Tomita, pelo incentivo constante, por sua paciência com minhas faltas e pelos valiosos ensinamentos que me dispensou. Dito de outro modo: Artur, muito obrigada por acreditar em mim, por dedicar tanto tempo a este projeto e por me estimular a ter diversas experiências acadêmicas ao longo do doutorado. Obrigada por escutar minhas idéias e por me dar liberdade de pensamento e escrita. Obrigada por compreender meus momentos de angústia e euforia e, mais do que isso, obrigada por estar presente quando eu mais precisei. Principalmente, obrigada por partilhar sua experiência e seu conhecimento com generosidade.

Agradeço à banca examinadora pelos comentários e sugestões. Particularmente, agradeço aos especialistas de outras áreas que se dispuseram a participar da comissão julgadora desta tese de doutorado, enriquecendo-a com seus apontamentos e observações.

Agradeço ao Instituto de Matemática e Estatística da Universidade de São Paulo pelas diversas oportunidades de me desenvolver profissionalmente. Tendo recebido (gratuitamente) tanto, não posso fazer outra coisa senão exercer a minha profissão com ética e excelência, em prol da sociedade brasileira. Agradeço também à CAPES e ao CNPq pelo apoio financeiro.

Agradeço aos meus amigos e colegas que, direta ou indiretamente, contribuíram com este trabalho. Em particular, agradeço ao Armando pelas dicas de $\mathrm{AT}_{\mathrm{EX}}$ e ao Marquinhos, por ter me apresentado o Geogebra.

Agradeço ao meu namorado, companheiro e parceiro de todas as horas por partilhar a vida comigo; por me permitir amar e ser amada, guiando-me pelos caminhos em que se encontra a 
viii

felicidade.

Agradeço à minha família pelos exemplos de conduta e superação que me inspiram desde a mais tenra idade. Em especial, agradeço aos meus pais e irmão pelo apoio emocional, moral e financeiro, pelo lar que construímos e pelo amor que nos une.

Sobretudo, agradeço a Deus por todas as bênçãos derramadas sobre minha vida. 
Assumindo a existência de $\mathfrak{c}$ ultrafiltros seletivos dois a dois incomparáveis (segundo a ordem de Rudin-Keisler) provamos que o grupo abeliano livre de cardinalidade $\mathfrak{c}$ admite uma topologia de grupo enumeravelmente compacta com uma seqüência não trivial convergente. Sob as mesmas hipóteses, mostramos que um grupo topológico abeliano quase livre de torção $(G,+, \tau)$ com $|G|=|\tau|=\mathfrak{c}$ admite uma topologia independente de $\tau$ que o torna um grupo topológico e caracterizamos algebricamente os grupos abelianos de não torção que têm cardinalidade $\mathfrak{c}$ e que admitem uma topologia de grupo enumeravelmente compacta (sem seqüências não triviais convergentes). Provamos, ainda, que o grupo abeliano livre de cardinalidade $\mathfrak{c}$ admite uma topologia de grupo que torna seu quadrado enumeravelmente compacto e construímos um semigrupo de Wallace cujo quadrado é, também, enumeravelmente compacto. Por fim, assumindo a existência de $2^{\mathfrak{c}}$ ultrafiltros seletivos, garantimos que se um grupo abeliano de não torção e cardinalidade $\mathfrak{c}$ admite uma topologia de grupo enumeravelmente compacta, então o mesmo admite $2^{\mathfrak{c}}$ topologias de grupo enumeravelmente compactas (duas a duas não homeomorfas).

Palavras-chave: compacidade enumerável, grupos topológicos, ultrafiltros seletivos 



\begin{abstract}
Assuming the existence of $\mathfrak{c}$ pairwise incomparable selective ultrafilters (according to the Rudin-Keisler ordering) we prove that the free abelian group of cardinality $\mathfrak{c}$ admits a countably compact group topology that contains a non-trivial convergent sequence. Under the same hypothesis, we show that an abelian almost torsion-free topological group $(G,+, \tau)$ with $|G|=|\tau|=\mathfrak{c}$ admits a group topology independent of $\tau$ and we algebraically characterize the nontorsion abelian groups of cardinality $\mathfrak{c}$ which admit a countably compact group topology (without non-trivial convergent sequences). We also prove that the free abelian group of cardinality $\mathfrak{c}$ admits a group topology that makes its square countably compact and we construct a Wallace's semigroup whose square is countably compact. Finally, assuming the existence of $2^{\mathfrak{c}}$ selective ultrafilters, we ensure that if a non-torsion abelian group of cardinality $\mathfrak{c}$ admits a countably compact group topology, then it admits $2^{\mathfrak{c}}$ (pairwise non-homeomorphic) countably compact group topologies.
\end{abstract}

Keywords: countable compactness, topological groups, selective ultrafilters 

1 Definições e resultados preliminares $\quad 5$

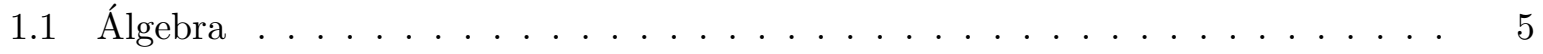

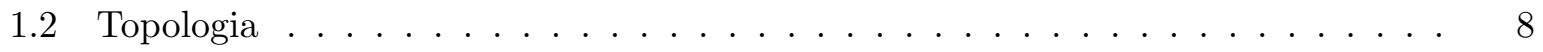

1.3 Grupos topológicos . . . . . . . . . . . . . . . . . . 10

1.4 Teoria dos conjuntos . . . . . . . . . . . . . . . . . . 11

1.5 Ultrafiltros seletivos e $p$-limites . . . . . . . . . . . . . . . . 12

2 Uma topologia enumeravelmente compacta com uma seqüência não trivial convergente no grupo abeliano livre de cardinalidade c que o torna um grupo $\begin{array}{ll}\text { topológico } & 15\end{array}$

2.1 Uma escolha adequada de seqüências . . . . . . . . . . . . . . . . 16

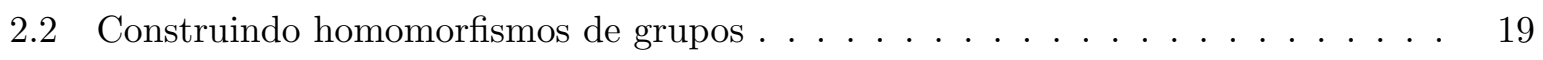

2.3 Explicitando a topologia . . . . . . . . . . . . . . . 27

3 Uma topologia de grupo enumeravelmente compacta sem seqüências não triviais convergentes na reta real $\quad \mathbf{2 9}$

3.1 Uma escolha adequada de seqüências . . . . . . . . . . . . . . . . . . . 29

3.2 Construindo homomorfismos de grupos . . . . . . . . . . . . . . . . . . 32

3.3 Explicitando a topologia . . . . . . . . . . . . . . . . . . 45 
4 Grupos abelianos quase livres de torção que têm cardinalidade c: topologias de grupo enumeravelmente compactas sem seqüências não triviais convergentes e topologias independentes $\quad 49$

4.1 Uma escolha adequada de seqüências . . . . . . . . . . . . . . . . . . . 50

4.2 Construindo homomorfismos de grupos . . . . . . . . . . . . . . . 55

4.3 Explicitando a topologia . . . . . . . . . . . . . . . . . . . 69

4.4 Topologias independentes . . . . . . . . . . . . . . . . . . . 71

5 Caracterização algébrica dos grupos abelianos de não torção que têm cardinalidade $\mathfrak{c}$ e que admitem uma topologia de grupo enumeravelmente $\begin{array}{ll}\text { compacta } & \mathbf{7 5}\end{array}$

5.1 Selecionando candidatos a pontos de acumulação . . . . . . . . . . . . . 76

5.2 Uma escolha adequada de seqüências . . . . . . . . . . . . . . . . . . 81

5.3 Construindo homomorfismos de grupos . . . . . . . . . . . . . . . . . . . . 88

5.4 Explicitando a topologia . . . . . . . . . . . . . . . . . . . . . . 104

5.5 Caracterização algébrica dos grupos abelianos de não torção que têm cardinalidade $\mathfrak{c}$ e que admitem uma topologia de grupo enumeravelmente compacta . . . . . . . 106

5.6 Aumentando o peso do grupo . . . . . . . . . . . . . . . . . . . . . . 107

6 Sobre o número de topologias de grupo enumeravelmente compactas que um

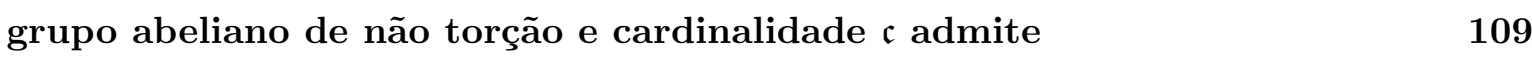

6.1 O invariante topológico $\mathcal{F} \ldots \ldots \ldots \ldots$. . . . . . . . . . . . 109

6.2 Recordando a notação adotada no capítulo $5 \ldots \ldots$. . . . . . . . . . 111

6.3 A construção de uma nova topologia . . . . . . . . . . . . . . . . . . . . 113

7 Construindo uma topologia de grupo no grupo abeliano livre de cardinalidade $\begin{array}{ll}\mathfrak{c} \text { que torna seu quadrado enumeravelmente compacto } & 117\end{array}$

7.1 Uma escolha adequada de seqüências . . . . . . . . . . . . . . . . . . . 118

7.2 Construindo homomorfismos de grupos . . . . . . . . . . . . . . . . . 127

7.3 Explicitando a topologia . . . . . . . . . . . . . . . . . . . . 145

7.4 Sobre o problema de Wallace . . . . . . . . . . . . . . . . . 146

8 Considerações finais $\quad 149$

8.1 Resultados obtidos . . . . . . . . . . . . . . . . . . . . . . . 149

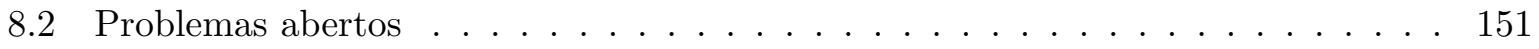


A Ultrafiltros seletivos

B Ultrafiltros seletivos e o axioma de Martin

Referências Bibliográficas

Índice Remissivo 

Em 1944, Halmos [23] mostrou que $(\mathbb{R},+)$ admite uma topologia que o torna um grupo topológico compacto e perguntou que outros grupos abelianos podem ser munidos de uma tal topologia. ${ }^{1}$ Esta questão contribuiu significativamente para o desenvolvimento da teoria dos grupos abelianos, particularmente através da introdução dos grupos algebricamente compactos por Kaplansky [27]. No fim da década de 50, Harrison [24] e Hulanicki [25] resolveram o problema proposto por Halmos. Eles mostraram que um grupo abeliano $G$ admite uma topologia que o torna um grupo compacto se, e somente se, $G$ é isomorfo a um produto de cópias de $\mathbb{R}$, de inteiros $p$-ádicos, de $p$-grupos quase cíclicos e de grupos cíclicos finitos onde, para cada número primo $p$, o número de cópias de $\mathbb{Z}_{p}(\infty)$ não excede o número de cópias de $\mathbb{R}$.

O teorema de Ivanovskij-Kuz'minov afirma que todo grupo topológico compacto é um espaço diádico (isto é, a imagem por uma aplicação contínua de um cubo de Cantor generalizado) e, portanto, todo grupo compacto infinito possui uma seqüência não trivial convergente. A questão da existência de grupos topológicos enumeravelmente compactos sem seqüências não triviais convergentes é bastante conhecida e não possui solução definitiva até o presente momento.

O primeiro exemplo de grupo enumeravelmente compacto (infinito) sem seqüências não triviais convergentes foi obtido por Hajnal e Juhász [22] em 1976, sob a hipótese do contínuo (CH). Quatro anos mais tarde, van Douwen [18] exibiu um outro exemplo com o auxílio do axioma de Martin (MA). Apenas recentemente, García-Ferreira, Tomita e Watson [20] mostraram que a existência de um grupo enumeravelmente compacto sem seqüências não triviais convergentes

\footnotetext{
${ }^{1}$ Neste trabalho, todas as topologias consideradas serão supostas $T_{1}$ - o que implica de Tychonoff, no caso de uma topologia de grupo.
} 
não depende de qualquer forma do axioma de Martin.

Nesta tese, apresentamos algumas construções de topologias enumeravelmente compactas em grupos abelianos de não torção que dependem apenas da existência de ultrafiltros seletivos. A existência de ultrafiltros seletivos é independente de ZFC. ${ }^{2}$ No apêndice B reproduzimos a prova de Blass [5] de que o axioma de Martin para ordens parciais enumeráveis ( $\mathrm{MA}_{\text {countable }}$ ) implica a existência de $2^{\mathfrak{c}}$ ultrafiltros seletivos. Todavia, a existência de $2^{\mathfrak{c}}$ ultrafiltros seletivos é compatível com a falha total de MA. ${ }^{3}$

Antes de descrevermos os resultados obtidos neste trabalho, gostaríamos de justificar nossa escolha de trabalhar com grupos abelianos de não torção. As técnicas que consideramos baseiamse na demonstração do teorema de Kertész-Szele, o qual afirma que todo grupo abeliano infinito admite uma topologia de Tychonoff não discreta que o torna um grupo topológico. Além disso, Dikranjan e Shakhmatov [14] provaram que nenhum grupo livre com mais de um elemento admite uma topologia de grupo enumeravelmente compacta e, em [15], os mesmos autores demonstraram que o grupo de permutações de um conjunto infinito $X$ também não admite uma tal topologia.

Sob condições mais fracas que a hipótese do contínuo generalizada (GCH), Castro-Pereira e Tomita [11] mostraram que um grupo abeliano de torção admite uma topologia de grupo enumeravelmente compacta se, e somente se, o mesmo admite uma topologia de grupo pseudocompacta. Assumindo a existência de um ultrafiltro seletivo, eles provaram que se $G$ é um grupo abeliano de torção tal que $|G|=\mathfrak{c}$, então $G$ admite uma topologia de grupo enumeravelmente compacta se, e somente se, $G$ é isomorfo a uma soma direta finita de grupos da forma $\bigoplus_{i \leq l_{p}} \mathbb{Z}_{p^{t_{p, i}}}^{\left(\theta_{p, i}\right)}$ onde $p$ é um número primo, $t_{p, 0}<t_{p, 1}<\ldots<t_{p, l_{p}}$ são números naturais e cada $\theta_{p, i}$ é um cardinal tal que se $i \leq l_{p}$ é o maior inteiros para o qual $\theta_{p, i}$ é infinito, então $\theta_{p, i}=\mathfrak{c}$.

Com respeito à sua estrutura, esta tese é composta por oito capítulos e dois apêndices. O apêncice A apresenta algumas caracterizações da seletividade, além de propriedades relacionadas a este conceito. O apêndice $\mathrm{B}$, por sua vez, traz uma prova de que $\mathrm{MA}_{\text {countable implica a existência }}$ de $2^{\mathfrak{c}}$ ultrafiltros seletivos.

O primeiro capítulo tem por objetivo listar os principais conceitos e resultados que serão utilizados ao longo do texto, bem como fixar algumas notações e terminologias que serão empregadas adiante.

\footnotetext{
${ }^{2}$ Existem $2^{\mathfrak{c}}$ ultrafiltros seletivos sob $\mathrm{CH}$ ou MA. Por outro lado, Shelah mostrou via forcing que existem modelos de ZFC sem p-pontos (e, em particular, sem ultrafiltros seletivos). Kunen [28] mostrou que não existem ultrafiltros seletivos no modelo dos reais aleatórios.

${ }^{3}$ No sentido de Baumgartner [2].
} 
No capítulo 2, assumimos a existência de $\mathfrak{c}$ ultrafiltros seletivos dois a dois incomparáveis (segundo a ordem de Rudin-Keisler) para construir uma topologia enumeravelmente compacta com uma seqüência não trivial convergente no grupo abeliano livre de cardinalidade $\mathfrak{c}$ que o torna um grupo topológico. ${ }^{4}$ García-Ferreira e Tomita obtiveram o mesmo resultado assumindo o axioma de Martin para ordens parciais $\sigma$-centradas $\left(\mathrm{MA}_{\sigma-\text { centered }}\right)$.

O capítulo 3 mostra que a reta real admite uma topologia de grupo enumeravelmente compacta sem seqüências não triviais convergentes. Embora este seja um resultado original - já que assumimos apenas a existência de ultrafiltros seletivos para obtê-lo — o capítulo em questão foi colocado neste trabalho com o único intuito de facilitar a compreensão do leitor, uma vez que versões mais gerais dos resultados nele apresentados encontram-se nos capítulos 4 e 5 .

O capítulo 4 generaliza as construções apresentadas no capítulo $3 .^{5}$ Explicitamente, mostramos que é possível munir um grupo abeliano quase livre de torção de cardinalidade $\mathfrak{c}$ de uma topologia que o torna um grupo topológico enumeravelmente compacto sem seqüências não triviais convergentes. Em 2002, Tkachenko e Yaschenko [37] usaram MA para mostrar que se $(G,+, \tau)$ é um grupo topológico abeliano quase livre de torção tal que $|G|=|\tau|=\mathfrak{c}$, então existe $\sigma$ uma topologia em $G$ independente de $\tau$ (isto é, tal que $\tau \cap \sigma$ é a topologia cofinita em $G)$ que torna $(G,+)$ um grupo topológico. Este resultado também decorre da nossa construção, o que mostra que não é necessária qualquer forma do axioma de Martin para obtê-lo.

Sob MA, Dikranjan e Tkachenko [17] caracterizaram algebricamente os grupos abelianos de não torção que têm cardinalidade $\mathfrak{c}$ e que admitem uma topologia de grupo enumeravelmente compacta. A saber, eles mostraram que um tal grupo $G$ admite uma tal topologia se, e somente se, $|G / T(G)|=\mathfrak{c}$ e, para quaisquer $n, d \in \mathbb{N}$ tais que $d \mid n$, o conjunto $d G[n]$ é finito ou tem cardinalidade c. No capítulo 5 , mostramos que esta caracterização algébrica dos grupos abelianos de não torção que têm cardinalidade $\mathfrak{c}$ e que admitem uma topologia de grupo enumeravelmente compacta permanece válida se assumirmos apenas a existência de $\mathfrak{c}$ ultrafiltros seletivos dois a dois incomparáveis.

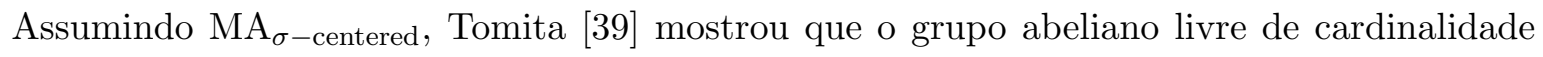
$\mathfrak{c}$ admite $\mathfrak{c}^{+}$topologias enumeravelmente compactas (duas a duas não homeomorfas) que o tornam um grupo topológico. No capítulo 6, provamos que a existência de $2^{\mathfrak{c}}$ ultrafiltros seletivos é suficiente para garantir que se um grupo abeliano de não torção e cardinalidade $\mathfrak{c}$

\footnotetext{
${ }^{4}$ Uma vez que dois grupos abelianos livres não enumeráveis de mesma cardinalidade são isomorfos, escreveremos "o grupo abeliano livre de cardinalidade $\kappa$ " em vez de "um grupo abeliano livre de cardinalidade $\kappa$ " onde $\kappa>\omega$ é um cardinal.

${ }^{5}$ Continuamos, aqui, assumindo a existência de $\mathfrak{c}$ ultrafiltros seletivos dois a dois incomparáveis.
} 
admite uma topologia de grupo enumeravelmente compacta, então o mesmo admite $2^{\mathfrak{c}}$ topologias enumeravelmente compactas (duas a duas não homeomorfas) que o tornam um grupo topológico. Isto melhora o resultado de [39] em duas direções: em primeiro lugar, estamos assumindo uma hipótese mais fraca que o axioma de Martin para ordens parciais $\sigma$-centradas; além disso, ampliamos a classe dos grupos para os quais temos o resultado.

Em 1998, Tomita [38] provou que o grupo abeliano livre de cardinalidade $\mathfrak{c}$ não admite uma topologia de grupo que torna sua $\omega$-ésima potência enumeravelmente compacta. No capítulo 7, assumimos a existência de $\mathfrak{c}$ ultrafiltros seletivos dois a dois incomparáveis e mostramos que existe uma topologia de grupo no grupo abeliano livre de cardinalidade $\mathfrak{c}$ que torna seu quadrado enumeravelmente compacto. Isto responde uma questão proposta por MadariagaGarcia e Tomita em [30] e por Tkachenko em [36].

Em 1952, Numakura [31] mostrou que todo semigrupo cancelativo (à direita e à esquerda) compacto é um grupo topológico. Em 1955, Wallace [45] perguntou se todo semigrupo cancelativo enumeravelmente compacto é um grupo topológico e esta questão permanece aberta em ZFC. Um contraexemplo para a pergunta de Wallace é conhecido como semigrupo de Wallace. Robbie e Svetlichny [32] responderam negativamente a pergunta de Wallace usando CH em 1996. Em 2007, Madariaga-Garcia e Tomita [30] construíram um semigrupo de Wallace assumindo a existência de $\mathfrak{c}$ ultrafiltros seletivos dois a dois incomparáveis. Nestas mesmas condições, mostramos que existe um semigrupo topológico cancelativo que não é um grupo topológico e cujo quadrado é enumeravelmente compacto. Isto responde a questão 4 proposta por Grant em [21].

Destacamos que os resultados apresentados no capítulo 2 encontram-se submetidos a uma revista com arbitragem (vide [6]), bem como os resultados apresentados nos capítulos 5 e 6 (vide [7]). Além disso, os resultados apresentados no capítulo 4 foram aceitos para publicação no Houston Journal of Mathematics (vide [8]) e uma versão mais fraca dos resultados apresentados no capítulo 7 foi publicada no Fundamenta Mathematicae (vide [9]).

Finalmente, o capítulo 8 traz algumas considerações finais a respeito deste trabalho. 
CAPÍTULO 1

\section{Definições e resultados preliminares}

Não temos o intuito de desenvolver neste capítulo toda a teoria necessária para a compreensão desta tese; seu propósito é fixar as nomenclaturas e notações que serão empregadas adiante, bem como enunciar alguns resultados que serão utilizados ao longo do texto. Nossas referências básicas são [33], [19], [1], e [29].

\section{1 Álgebra}

Seja $(G,+)$ um grupo abeliano. Denotaremos por $0_{G}$ o seu elemento neutro e por $-x$ o inverso de $x \in G$. Observamos desde já que alguns abusos de notação serão cometidos ao longo deste trabalho. Por exemplo, quando não houver risco à interpretação, representaremos o grupo $(G,+)$ e seu elemento neutro $0_{G}$ por $G$ e 0 , respectivamente.

Dado $X \subset G$, denotaremos por $\langle X\rangle$ o subgrupo de $G$ gerado por $X$. Se $X=\{x\}$, o grupo cíclico gerado por $x$ será denotado por $\langle x\rangle$. A ordem de um subgrupo $H$ de $G$ será representada por $|H|$. Se $|\langle x\rangle| \geq \omega$, diremos que $x$ tem ordem infinita. Caso contrário, a ordem de $x$ será dada por o $(x)=|\langle x\rangle|$. Observamos que se $m, n \in \mathbb{N} \backslash\{0\}$ e o $(x)=n$, então o $(m x)=n / \operatorname{mdc}(m, n)$.

Proposição 1.1.1. Sejam $F$ e $G$ grupos abelianos, $H$ um subgrupo de $G, f: H \rightarrow F$ um homomorfismo de grupos, $x \in G$ e $y \in F$. É possivel estender $f$ a um homomorfismo de grupos 
$\tilde{f}:\langle H \cup\{x\}\rangle \rightarrow F$ satisfazendo $\tilde{f}(x)=y$ se, e somente se, uma das seguintes condições é satisfeita:

(i) $n x \notin H$, para todo $n \in \mathbb{N} \backslash\{0\}$;

(ii) $\{n \in \mathbb{N} \backslash\{0\}: n x \in H\} \neq \emptyset$ e $f(m x)=m y$, onde $m=\min \{n \in \mathbb{N} \backslash\{0\}: n x \in H\}$.

Demonstração. Ver lema 1.1 .5 de [1].

Dizemos que $S \subset G$ é independente se $0 \notin S$ e se, para quaisquer $s_{1}, \ldots, s_{n} \in S$ dois a dois distintos e quaisquer $m_{1}, \ldots, m_{n} \in \mathbb{Z}$ tais que $m_{1} s_{1}+\ldots+m_{n} s_{n}=0$, tivermos que $m_{1} s_{1}=\ldots=m_{n} s_{n}=0$.

Corolário 1.1.2. Sejam $S$ um subconjunto independente de $G$ e $H$ um grupo. Suponhamos que para cada $x \in S$ exista $\phi(x) \in H$ tal que se $x$ tem ordem finita, então o $(\phi(x)) \mid \mathrm{o}(x)$. É possível estender $\phi$ a um homomorfismo de grupos de $\langle S\rangle$ em $H$.

O subgrupo de torção de $G$ é denotado por $T(G)$ e é constituído de todos os elementos de $G$ que têm ordem finita. Dizemos que $G$ é de não torção (ou que $G$ é não periódico) se $G \neq T(G$ ). Caso contrário, $G$ é dito de torção. Se existe $n \in \mathbb{N}$ tal que o $(x) \leq n$ para todo $x \in G$, dizemos que $G$ é de torção limitada. Por fim, $G$ é dito quase livre de torção se $|G[n]|<\omega$ para todo $n \in \mathbb{N} \backslash\{0\}$, onde $G[n]=\{x \in G: n x=0\}$.

Teorema 1.1.3. (Prüfer-Baer) Todo grupo abeliano de torção limitada é isomorfo a uma soma direta de grupos cíclicos cujas ordens são finitas e limitadas.

Demonstração. Ver 4.3.5 de [33].

Se $X$ é um conjunto, escreveremos $G^{X}$ para denotar o produto $\prod_{x \in X} G_{x}$ onde $G_{x}=G$, para cada $x \in X$. Escreveremos, ainda, $G^{(X)}$ para representar a soma direta $\bigoplus_{x \in X} G_{x}$ onde $G_{x}=G$, para cada $x \in X$. Se $J \in G^{X}$, o suporte de $J$ é o conjunto supp $J=\{x \in X: J(x) \neq 0\}$.

Dizemos que $G$ é abeliano livre se existe $X$ um subconjunto não vazio de $G$ tal que cada função definida em $X$ a valores em um grupo abeliano $H$ se estende a um único homomorfismo de grupos de $G$ em $H{ }^{1}$

Proposição 1.1.4. (i) Se um subconjunto $X$ de $G$ testemunha que $G$ é um grupo abeliano livre, então $G$ é isomorfo à soma direta dos subgrupos cíclicos infinitos $\langle x\rangle$, onde $x \in X$.

\footnotetext{
${ }^{1}$ Neste caso, dizemos que $X$ testemunha que $G$ é um grupo abeliano livre.
} 
(ii) O conjunto $X$ testemunha que $\mathbb{Z}^{(X)}$ é um grupo abeliano livre.

Demonstração. Ver 2.3.8 de [33].

Corolário 1.1.5. Seja $X$ um conjunto de cardinalidade $\kappa>\omega$. Se $G$ é um grupo abeliano livre de cardinalidade $\kappa$, então $G$ é isomorfo a $\mathbb{Z}^{(X)}$.

Demonstração. Como $|G|=\kappa>\omega$, existe $Y$ um subconjunto de $G$ de cardinalidade $\kappa$ que testemunha que $G$ é um grupo abeliano livre. Além disso, $X$ testemunha que $\mathbb{Z}^{(X)}$ é um grupo abeliano livre. Uma vez que $|X|=|Y|$, o argumento apresentado na proposição 2.1.4 de [33] nos permite concluir que $G$ é isomorfo a $\mathbb{Z}^{(X)}$.

Dizemos que $G$ é divisível se, para cada $g \in G$ e cada $n \in \mathbb{N} \backslash\{0\}$, a equação $n x=g$ tem uma solução $x \in G$.

Proposição 1.1.6. (Baer) Sejam $H$ um subgrupo do grupo abeliano $G, D$ um grupo (abeliano) divisivel e $f: H \rightarrow D$ um homomorfismo de grupos. Existe $\tilde{f}: G \rightarrow D$ um homomorfismo de grupos tal que $\tilde{f} \uparrow_{H}=f$.

Demonstração. Ver 4.1.2 de [33].

Teorema 1.1.7. Um grupo abeliano é divisível se, e somente se, é isomorfo a uma soma direta de cópias de $\mathbb{Q}$ e de grupos quase-cíclicos. ${ }^{2}$

Demonstração. Ver 4.1.5 de [33].

Teorema 1.1.8. Todo grupo abeliano é isomorfo a um subgrupo de um grupo divisível.

Demonstração. Ver 4.1.6 de [33].

A circunferência unitária centrada na origem do plano complexo munida da multiplicação usual de números complexos é um exemplo de grupo abeliano divisível, o qual será denotado por $\mathbb{T}$.

Se $w \in \mathbb{T}$ e $n \in \mathbb{N} \backslash\{0\}$, então $z \in \mathbb{T}$ é uma raiz $n$-ésima de $w$ se $z^{n}=w$. No caso particular em que $w=1$, um tal $z$ é denominado raiz $n$-ésima da unidade. É fácil ver que as raízes $n$-ésimas da unidade são precisamente os números complexos da forma

$$
e^{\frac{2 \pi i k}{n}}=\cos \left(\frac{2 \pi k}{n}\right)+i \operatorname{sen}\left(\frac{2 \pi k}{n}\right)
$$

\footnotetext{
${ }^{2}$ Dado $p$ um número primo, o subgrupo de $\mathbb{Q} / \mathbb{Z}$ gerado pelo conjunto $\left\{1 / p^{n}+\mathbb{Z}: n \in \omega\right\}$ é denominado $p$-grupo quase-cíclico e é denotado por $\mathbb{Z}\left(p^{\infty}\right)$.
} 
onde $k \in\{0,1, \ldots, n-1\}$. Também não é difícil notar que para cada $w \in \mathbb{T}$, existem $n$ raízes $n$-ésimas de $w$ e que o conjunto das raízes $n$-ésimas de $w$ é obtido através de uma translação do conjunto das raízes $n$-ésimas da unidade por uma raíz $n$-ésima de $w .^{3}$

\subsection{Topologia}

Seja $(X, \tau)$ um espaço topológico. ${ }^{4}$ Para cada $x \in X$, denotaremos por $\tau_{x}$ a família de todas as vizinhanças abertas de $x$ em $X$.

Dizemos que $X$ é enumeravelmente compacto se todo recobrimento aberto enumerável de $X$ admite um sub-recobrimento finito.

Proposição 1.2.1. Todo subconjunto fechado de um espaço enumeravelmente compacto é enumeravelmente compacto.

Demonstração. Ver 3.10 .4 de [19].

Proposição 1.2.2. A imagem de um espaço enumeravelmente compacto por uma aplicação contínua é enumeravelmente compacta.

Demonstração. Ver 3.10 .5 de [19].

Dizemos que $X$ é pseudocompacto se toda função contínua a valores reais definida em $X$ é limitada.

Proposição 1.2.3. Todo espaço topológico enumeravelmente compacto é pseudocompacto.

Demonstração. Ver 3.10 .20 de [19].

Dado $A \subset X$, denotaremos por $\bar{A}$ o fecho de $A$ em $X$. Dizemos que $x \in X$ é um ponto de acumulação de $A$ se $(U \backslash\{x\}) \cap A \neq \emptyset$, para cada $U \in \tau_{x}$.

Proposição 1.2.4. Um espaço topológico X é enumeravelmente compacto se, e somente se, todo subconjunto infinito de $X$ tem um ponto de acumulação.

Demonstração. Ver 3.10 .3 de [19].

\footnotetext{
${ }^{3}$ Portanto, assim como as raízes $n$-ésimas da unidade, as raízes $n$-ésimas de $w$ se encontram simetricamente distribuídas em $\mathbb{T}$.

${ }^{4}$ Quando não houver risco à interpretação, representaremos o espaço topológico $(X, \tau)$ por $X$, simplesmente.
} 
O caráter de um ponto $x$ no espaço topológico $X$ é dado por

$$
\chi(x, X)=\min \{|\mathcal{B}(x)|: \mathcal{B}(x) \text { é um sistema fundamental de vizinhanças para } x \text { em } X\}
$$

e o caráter de $X$ é dado por

$$
\chi(X)=\sup \{\chi(x, X): x \in X\}+\omega .
$$

O peso de $X$, por sua vez, é dado por

$$
w(X)=\min \{|\mathcal{B}|: \mathcal{B} \text { é uma base de abertos para } X\}+\omega .
$$

Observamos que $|\tau| \leq 2^{\omega(X)}$.

Teorema 1.2.5. (Kronecker) Se $\zeta \in \mathbb{R} \backslash \mathbb{Q}$, então $\{(x+\mathbb{Z}, \zeta x+\mathbb{Z}): x \in \mathbb{R}\}$ é um subconjunto denso do toro $\mathbb{T}^{2}$.

Demonstração. Ver 4.13 na seção 4 do capítulo 1 de [10].

Sejam $(M, \delta)$ um espaço métrico e $A$ um subconjunto de $M$. Se $A \neq \emptyset$, o diâmetro de $A$ é definido por $\delta(A)=\sup \{\delta(x, y): x, y \in A\}$. Se $A=\emptyset$, então $\delta(A)=0$. Dado $\epsilon>0$, dizemos que $A \subset M$ é $\epsilon$-denso se, para cada $y \in M$, existe $a \in A$ tal que $\delta(y, a)<\epsilon$.

Corolário 1.2.6. Sejam $\epsilon>0$ e $\zeta \in \mathbb{R} \backslash \mathbb{Q}$. Existe $l=l(\zeta, \epsilon)>0$ tal que se $I$ é um intervalo aberto de $\mathbb{R}$ de comprimento maior que l, então $\{(x+\mathbb{Z}, \zeta x+\mathbb{Z}): x \in I\}$ é $\epsilon$-denso no toro $\mathbb{T}^{2}$.

Demonstração. Seja $\mathcal{C}$ uma cobertura finita do toro $\mathbb{T}^{2}$ por bolas abertas de raio $\epsilon / 2$. Como $\zeta \in \mathbb{R} \backslash \mathbb{Q}$, do teorema 1.2.5 decorre que $\{(x+\mathbb{Z}, \zeta x+\mathbb{Z}): x \in \mathbb{R}\}$ é denso em $\mathbb{T}^{2}$. Logo, para cada $U \in \mathcal{C}$ existe $x_{U} \in \mathbb{R}$ tal que $\left(x_{U}+\mathbb{Z}, \zeta x_{U}+\mathbb{Z}\right) \in U$. Temos que $\left\{\left(x_{U}+\mathbb{Z}, \zeta x_{U}+\mathbb{Z}\right): U \in \mathcal{C}\right\}$ é um subconjunto $\epsilon$-denso do toro $\mathbb{T}^{2}$. Portanto, se $J$ é um intervalo aberto de $\mathbb{R}$ que contém $\left\{x_{U} \in \mathbb{R}: U \in \mathcal{C}\right\}$, então o conjunto $\{(x+\mathbb{Z}, \zeta x+\mathbb{Z}): x \in J\}$ é $\epsilon$-denso em $\mathbb{T}^{2}$. Seja $l$ o comprimento de $J$. Observamos que se $I$ é um intervalo aberto de $\mathbb{R}$ de comprimento maior que $l$, então existe $a \in \mathbb{R}$ tal que $J+a \subset I$. Como as translações em $\mathbb{T}^{2}$ são isometrias, concluímos que $\{(x+\mathbb{Z}, \zeta x+\mathbb{Z}): x \in I\}$ é $\epsilon$-denso em $\mathbb{T}^{2}$. 


\subsection{Grupos topológicos}

Um semigrupo é um conjunto munido de uma operação binária associativa. Dizemos que um semigrupo $(S, \cdot)$ é um semigrupo topológico se existe uma topologia no conjunto $S$ que torna a operação $: S \times S \rightarrow S$ contínua, onde consideramos $S \times S$ munido da topologia produto.

Um grupo topológico é uma tripla $(G, \cdot, \tau)$, onde $(G, \cdot)$ é um grupo e $\tau$ é uma topologia em $G$ que torna as aplicações

$$
\begin{aligned}
G \times G & \rightarrow G \\
(x, y) & \mapsto x \cdot y
\end{aligned}
$$

$\mathrm{e}$

$$
\begin{aligned}
G & \rightarrow G \\
x & \mapsto x^{-1}
\end{aligned}
$$

contínuas. ${ }^{5}$

É evidente que todo grupo topológico é, em particular, um semigrupo topológico. Observamos, ainda, que todo subgrupo (no sentido algébrico) de um grupo topológico é um grupo topológico, se o considerarmos munido da topologia de subespaço.

Exemplo 1.3.1. O grupo $\mathbb{T}$ munido da topologia de subespaço induzida por $\mathbb{R}^{2}$ é um grupo topológico.

Exemplo 1.3.2. O grupo $\mathbb{R} / \mathbb{Z}$ munido da topologia quociente induzida pela projeção de $\mathbb{R}$ em $\mathbb{R} / \mathbb{Z}$ é um grupo topológico.

Sejam $G$ e $H$ grupos topológicos. Uma aplicação $f: G \rightarrow H$ é dita um isomorfismo de grupos topológicos se $f$ é um isomorfismo de grupos entre os grupos (no sentido algébrico) $G$ e $H$ e se $f$ é um homeomorfismo entre os espaços topológicos $G$ e $H$. Dizemos que dois grupos topológicos são isomorfos se existe um isomorfismo de grupos topológicos entre eles.

Proposição 1.3.3. Os grupos topológicos $\mathbb{T} e \mathbb{R} / \mathbb{Z}$ são isomorfos.

Demonstração. A aplicação

$$
\begin{aligned}
\varphi: \mathbb{R} / \mathbb{Z} & \rightarrow \mathbb{T} \\
x+\mathbb{Z} & \mapsto e^{2 \pi i x}
\end{aligned}
$$

está bem definida, uma vez que se $x, y \in \mathbb{R}$ são tais que $x+\mathbb{Z}=y+\mathbb{Z}$, então existe $n \in \mathbb{Z}$ tal

\footnotetext{
${ }^{5}$ Novamente, estamos considerando $G \times G$ munido da topologia produto.
} 
que $x=y+n$ e, portanto,

$$
\varphi(x+\mathbb{Z})=\varphi((y+n)+\mathbb{Z})=e^{2 \pi i(y+n)}=e^{2 \pi i y} e^{2 \pi i n}=e^{2 \pi i y}=\varphi(y+\mathbb{Z}) .
$$

Também não é difícil mostrar que $\varphi$ é um homomorfismo de grupos. Com efeito,

$$
\varphi((x+\mathbb{Z})+(y+\mathbb{Z}))=\varphi((x+y)+\mathbb{Z})=e^{2 \pi i(x+y)}=e^{2 \pi i x} e^{2 \pi i y}=\varphi(x+\mathbb{Z}) \varphi(y+\mathbb{Z})
$$

quaisquer que sejam $x, y \in \mathbb{R}$. Por fim, a prova de que $\varphi$ é um homeomorfismo pode ser encontrada em [19] (ver Exemplo 2.4.11).

No que segue, identificaremos os grupos topológicos $\mathbb{T}$ e $\mathbb{R} / \mathbb{Z}$ e denotaremos por $\delta$ a métrica em $\mathbb{R} / \mathbb{Z}$ dada por

$$
\delta(x+\mathbb{Z}, y+\mathbb{Z})=\min \{|x-y+n|: n \in \mathbb{Z}\}
$$

quaisquer que sejam $x, y \in \mathbb{R}$.

Proposição 1.3.4. Seja $\left\{G_{\alpha}: \lambda \in \Lambda\right\}$ uma família de grupos topológicos. O conjunto $\prod_{\lambda \in \Lambda} G_{\lambda}$ com a operação usual de produto de grupos e munido da topologia produto é um grupo topológico.

Demonstração. Ver 1.2.7 de [1].

\subsection{Teoria dos conjuntos}

Sejam $\alpha$ e $\beta$ ordinais e $f: \alpha \rightarrow \beta$. Dizemos que $f$ é uma aplicação cofinal se $f[\alpha]$ é ilimitado em $\beta$. A cofinalidade de um ordinal $\beta$ é denotada por $\operatorname{cf}(\beta)$ e é definida como sendo o menor ordinal $\alpha$ tal que existe uma aplicação cofinal $f: \alpha \rightarrow \beta$.

Um cardinal $\kappa$ é dito regular se $\operatorname{cf}(\kappa)=\kappa$. Caso contrário, $\kappa$ é dito singular.

Lema 1.4.1. (König) Se $\kappa$ é um cardinal infinito e $\operatorname{cf}(\kappa) \leq \lambda$, então $\kappa^{\lambda}>\kappa$.

Demonstração. Ver 10.40 de [29].

Temos que $\mathfrak{c}^{\omega}=\mathfrak{c}$. Do lema de König segue que $\operatorname{cf}(\mathfrak{c})>\omega$.

Uma família $\mathcal{A}$ de conjuntos é dita um $\Delta$-sistema se existe um conjunto $r$ - denominado raiz do $\Delta$-sistema - tal que $a \cap b=r$, quaisquer que sejam $a$ e $b$ elementos distintos de $\mathcal{A}$. 
Proposição 1.4.2. Seja $\kappa$ um cardinal infinito. Suponha que $\theta>\kappa$ seja um cardinal regular tal que $\forall \alpha<\theta\left(\left|\alpha^{<\kappa}\right|<\theta\right)$. Assuma que $|\mathcal{A}| \geq \theta$ e que $\forall x \in \mathcal{A}(|x|<\kappa)$. Existe $\mathcal{B} \subset \mathcal{A}$ tal que $|\mathcal{B}|=\theta$ e $\mathcal{B}$ constitui um $\Delta$-sistema.

Demonstração. Ver 1.6 de [29].

\subsection{Ultrafiltros seletivos e $p$-limites}

Um filtro sobre $\omega$ é uma família não vazia $\mathfrak{F}$ de subconjuntos de $\omega$ com as seguintes propriedades:

(1) $\emptyset \notin \mathfrak{F}$

(2) Se $A, B \in \mathfrak{F}$, então $A \cap B \in \mathfrak{F}$;

(3) Se $A \in \mathfrak{F}$ e $B \subset \omega$ é tal que $A \subset B$, então $B \in \mathfrak{F}$.

Uma base para um filtro sobre $\omega$ é uma família não vazia $\mathfrak{B}$ de subconjuntos de $\omega$ com as seguintes propriedades:

(1) $\emptyset \notin \mathfrak{B}$;

(2) Se $A, B \in \mathfrak{B}$, então existe $C \in \mathfrak{B}$ tal que $A \cap B \supset C$.

Se $\mathfrak{B}$ é uma base para um filtro sobre $\omega$, então $\{X \subset \omega: \exists B \in \mathfrak{B}$ tal que $X \supset B\}$ é um filtro sobre $\omega$, denominado filtro gerado por $\mathfrak{B}$.

Um ultrafiltro sobre $\omega$ é um filtro sobre $\omega$ maximal com respeito à inclusão. Dizemos que um ultrafiltro $u$ sobre $\omega$ é principal se existe $n \in \omega$ tal que $u=\{A \subset \omega: n \in A\}$; caso contrário, $u$ é denominado livre.

Proposição 1.5.1. Um filtro $\mathfrak{F}$ sobre $\omega$ é um ultrafiltro se, e somente se, para cada $X \subset \omega$, $X \in \mathfrak{F}$ ou $\omega \backslash X \in \mathfrak{F}$.

Demonstração. Ver lema 7.4 de [26].

Denotaremos por $\beta \omega$ a compactificação de Stone-Čech do espaço discreto $\omega$, a qual será identificada com o conjunto de todos os ultrafiltros sobre $\omega$ munido da topologia que tem $\{V(A): A \subset \omega\}$ como base de abertos, onde $V(A)=\{u: u$ é um ultrafiltro sobre $\omega$ e $A \in u\}$. 
Nesta identificação, o espaço discreto $\omega$ corresponde à família dos ultrafiltros principais sobre $\omega$. Representaremos por $\omega^{*}$ a família dos ultrafiltros livres sobre $\omega$ (isto é, $\omega^{*}=\beta \omega \backslash \omega$ ).

Dada $f: \omega \rightarrow \omega$, a função $\beta f: \beta \omega \rightarrow \beta \omega$ definida por

$$
\beta f(p)=\left\{A \subset \omega: f^{-1}(A) \in p\right\}
$$

é denominada a extensão de Stone- $\check{C} e c h$ de $f$.

Definição 1.5.2. Dados $p, q \in \omega^{*}$, dizemos que $p \leq_{R K} q$ se existe uma função $f: \omega \rightarrow \omega$ tal que $\beta f(q)=p$, onde $\beta f$ denota a extensão de Stone-Čech de $f$. A pré-ordem $\leq_{R K}$ em $\omega^{*}$ é chamada de ordem de Rudin-Keisler. ${ }^{6}$

Definição 1.5.3. Um ultrafiltro livre p sobre $\omega$ é dito seletivo se para cada partição $\left\{P_{n}: n \in \omega\right\}$ de $\omega$, existe $m \in \omega$ tal que $P_{m} \in p$ ou existe $B \in p$ tal que $\left|B \cap P_{n}\right| \leq 1$, qualquer que seja $n \in \omega .^{7}$

O próximo lema será fortemente utilizado ao longo deste trabalho. Sua demonstração encontra-se no apêndice A.

Lema 1.5.4. [40] Seja $\left\{p_{j}: j \in \omega\right\}$ uma família de ultrafiltros seletivos dois a dois incomparáveis (segundo a ordem de Rudin-Keisler). Para cada $j \in \omega$, seja $\left\{a_{k}^{j}: k \in \omega\right\} \in p_{j}$ uma seqüência estritamente crescente de números naturais tal que $k<a_{k}^{j}$, qualquer que seja $k \in \omega$. Existe $\left\{I_{j}: j \in \omega\right\}$ uma familia de subconjuntos de $\omega$ dois a dois disjuntos tal que:

(i) $\left\{a_{k}^{j}: k \in I_{j}\right\} \in p_{j}$, para cada $j \in \omega$;

(ii) $\left\{\left[k, a_{k}^{j}\right]: j \in \omega, k \in I_{j}\right\}$ é uma familia de intervalos de $\omega$ dois a dois disjuntos.

A seguinte definição foi introduzida em [3] e está bastante ligada à noção de compacidade enumerável.

Definição 1.5.5. Sejam $p \in \omega^{*} e\left\{x_{n}: n \in \omega\right\}$ uma seqüência em um espaço topológico $X$. Dizemos que $x \in X$ é $p$-limite de $\left\{x_{n}: n \in \omega\right\}$ se, para cada vizinhança $U$ de $x$, o conjunto $\left\{n \in \omega: x_{n} \in U\right\}$ é um elemento de $p$. Neste caso, escrevemos $x=p-\lim \left\{x_{n}: n \in \omega\right\}$.

Proposição 1.5.6. Se $p \in \omega^{*} e\left\{X_{i}: i \in I\right\}$ é uma família de espaços topológicos, então $\left(y_{i}\right)_{i \in I} \in \prod_{i \in I} X_{i}$ é p-limite da seqüência $\left\{\left(x_{i}^{n}\right)_{i \in I}: n \in \omega\right\} \subset \prod_{i \in I} X_{i}$ se, e somente se, $y_{i}=p-\lim \left\{x_{i}^{n}: n \in \omega\right\}$, para todo $i \in I$.

\footnotetext{
${ }^{6}$ Por "pré-ordem", entendemos uma relação reflexiva e transitiva.

${ }^{7}$ Dizer que $p \in \omega^{*}$ é seletivo é o mesmo que dizer que, para cada partição $\left\{P_{n}: n \in \omega\right\}$ de $\omega$, existe $m \in \omega$ tal que $P_{m} \in p$ ou existe $B \in p$ tal que $\left|B \cap P_{n}\right|=1$, qualquer que seja $n \in \omega$.
} 
Demonstração. Suponhamos que $\left(y_{i}\right)_{i \in I}=p-\lim \left\{\left(x_{i}^{n}\right)_{i \in I}: n \in \omega\right\}$ e tomemos $i \in I$. Se $\Omega_{i}$ é uma vizinhança de $y_{i}$ em $X_{i}$, então $\left\{n \in \omega: x_{i}^{n} \in \Omega_{i}\right\}=\left\{n \in \omega:\left(x_{i}^{n}\right)_{i \in I} \in \Omega\right\} \in p$, onde $\Omega=\prod_{j \in I} \Omega_{j}$ e $\Omega_{j}=X_{j}$, para todo $j \in I \backslash\{i\}$. Portanto, $y_{i}=p-\lim \left\{x_{i}^{n}: n \in \omega\right\}$.

Reciprocamente, suponhamos que $y_{i}=p-\lim \left\{x_{i}^{n}: n \in \omega\right\}$, para todo $i \in I$. Sejam $\Omega=\prod_{i \in I} \Omega_{i}$ um aberto básico de $\prod_{i \in I} X_{i}$ tal que $\left(y_{i}\right)_{i \in I} \in \Omega$ e $F=\left\{i \in I: \Omega_{i} \neq X_{i}\right\}$. Observamos que $F$ é um subconjunto finito de $I$ e que, portanto, $\left\{n \in \omega:\left(x_{i}^{n}\right)_{i \in I} \in \Omega\right\}=$ $\cap_{i \in F}\left\{n \in \omega: x_{i}^{n} \in \Omega_{i}\right\} \in p$. Logo, $\left(y_{i}\right)_{i \in I}=p-\lim \left\{\left(x_{i}^{n}\right)_{i \in I}: n \in \omega\right\}$.

Proposição 1.5.7. Sejam G um grupo topológico e $p \in \omega^{*}$.

(i) Se $\left\{x_{n}: n \in \omega\right\}$ e $\left\{y_{n}: n \in \omega\right\}$ são seqüências em $G$ e $x, y \in G$ são tais que $x=p-\lim \left\{x_{n}: n \in \omega\right\}$ e $y=p-\lim \left\{y_{n}: n \in \omega\right\}$, então $x+y=p-\lim \left\{x_{n}+y_{n}: n \in \omega\right\} ;$

(ii) Se $\left\{x_{n}: n \in \omega\right\}$ é uma seqüência em $G$ e $x \in G$ são tais que $x=p-\lim \left\{x_{n}: n \in \omega\right\}$, então $-x=p-\lim \left\{-x_{n}: n \in \omega\right\}$.

Demonstração. (i) Seja $V$ uma vizinhança aberta de $x+y$ em $G$. Uma vez que $+: G \times G \rightarrow G$ é contínua, existem $V_{x}$ e $V_{y}$ subconjuntos abertos de $G$ tais que $x \in V_{x}, y \in V_{y}$ e $V_{x}+V_{y} \subset V$. Como $x=p-\lim \left\{x_{n}: n \in \omega\right\}$ e $y=p-\lim \left\{y_{n}: n \in \omega\right\}$, os conjuntos $A_{x}=\left\{n \in \omega: x_{n} \in V_{x}\right\}$ e $A_{y}=\left\{n \in \omega: y_{n} \in V_{y}\right\}$ são elementos de $p$. Temos que $\left\{n \in \omega: x_{n}+y_{n} \in V\right\} \supset A_{x} \cap A_{y}$ e, portanto, $\left\{n \in \omega: x_{n}+y_{n} \in V\right\} \in p$. Logo, $x+y=p-\lim \left\{x_{n}+y_{n}: n \in \omega\right\}$.

(ii) Análoga à de (i), uma vez que a aplicação que associa cada elemento $x$ de $G$ ao seu oposto $-x$ é contínua.

Proposição 1.5.8. Se $p \in \omega^{*}$ e $X$ é um espaço topológico compacto, então toda seqüência em $X$ admite p-limite.

Demonstração. Seja $\left\{x_{n}: n \in \omega\right\}$ uma seqüência em $X$. Como $p$ é um filtro sobre $\omega$, a família $\left\{\left\{x_{n}: n \in A\right\}: A \in p\right\}$ tem a propriedade da intersecção finita. Da compacidade de $X$ decorre que $\bigcap_{A \in p} \overline{\left\{x_{n}: n \in A\right\}} \neq \emptyset$. Afirmamos que se $x \in \bigcap_{A \in p} \overline{\left\{x_{n}: n \in A\right\}}$, então $x=p-\lim \left\{x_{n}: n \in \omega\right\}$. Com efeito, seja $U$ uma vizinhança de $x$ em $X$. O conjunto $\left\{n \in \omega: x_{n} \in U\right\} \in p$, uma vez que $p$ é um ultrafiltro sobre $\omega$ e $x \in \overline{\left\{x_{n}: n \in A\right\}}$ para todo $A \in p$. 
CAPÍTULO 2

\section{Uma topologia enumeravelmente compacta com uma seqüência não trivial convergente no grupo abeliano livre de cardinalidade $\mathfrak{c}$ que o torna um grupo topológico}

Neste capítulo, assumiremos a existência de $\mathfrak{c}$ ultrafiltros seletivos dois a dois incomparáveis (segundo a ordem de Rudin-Keisler) para mostrar que o grupo abeliano livre de cardinalidade $\mathfrak{c}$ admite uma topologia enumeravelmente compacta com uma seqüência não trivial convergente que o torna um grupo topológico. ${ }^{1}$

Para cada $J \in \mathbb{Z}^{(\mathfrak{c})} \backslash\{0\}$ construiremos um homomorfismo de grupos $\phi_{J}: \mathbb{Z}^{(\mathfrak{c})} \rightarrow \mathbb{T}$ de modo que o produto diagonal da família $\left\{\phi_{J}: J \in \mathbb{Z}^{(\mathfrak{c})} \backslash\{0\}\right\}$ seja injetor e que a imagem de $\mathbb{Z}^{(\mathfrak{c})}$ por esta aplicação tenha as propriedades desejadas quando munida da topologia de subespaço induzida por $\mathbb{T}^{\mathbb{Z}^{(\mathfrak{c})} \backslash\{0\}}$.

As aplicações $\phi_{J}$ serão estabelecidas de forma independente, em dois estágios: começaremos definindo $\phi_{J}$ em um subgrupo enumerável de $\mathbb{Z}^{(\mathfrak{c})}$ e, em seguida, estenderemos este homomorfismo parcial a $\mathbb{Z}^{(\mathfrak{c})}$. A primeira etapa será feita por indução onde, em cada passo, aproximaremos os valores de $\phi_{J}$ por arcos abertos não vazios de $\mathbb{T}$. Esta aproximação por arcos exige que as seqüências consideradas apresentem uma certa liberdade para terem atribuídos a si pontos de acumulação pré-determinados, o que justifica a próxima seção.

\footnotetext{
${ }^{1}$ Sejam $X$ um conjunto e $f: \omega \rightarrow X$. Dizemos que $f$ é trivial se existe $n_{0} \in \omega$ tal que $f(n)=f\left(n_{0}\right)$ para todo $n \geq n_{0}$.
} 


\subsection{Uma escolha adequada de seqüências}

Se $J \in \mathbb{Z}^{(\mathfrak{c})}$, então

$$
J=\sum_{\mu \in \operatorname{supp} J} J(\mu) \cdot \chi_{\mu}
$$

onde $\chi_{\mu}: \mathfrak{c} \rightarrow \mathbb{Z}$ é tal que

$$
\chi_{\mu}(\xi)=\left\{\begin{array}{lll}
1 & \text { se } & \xi=\mu \\
0 & \text { se } & \xi \neq \mu
\end{array}\right.
$$

Definimos

$$
|J|=\max \{|J(\mu)|: \mu \in \operatorname{supp} J\}
$$

e

$$
\|J\|=\sum_{\mu \in \operatorname{supp} J}|J(\mu)| .
$$

Denotaremos por $\mathcal{F}$ o conjunto de todas as funções $f: \omega \rightarrow \mathbb{Z}^{(\mathfrak{c})}$ que satisfazem uma das seguintes condições:

(1) $\|f(n)\|>n$, para todo $n \in \omega$;

(2) (a) $\operatorname{supp} f(n) \cap \omega=\emptyset$, para todo $n \in \omega$;

(b) $\operatorname{supp} f(n) \backslash \cup_{m<n} \operatorname{supp} f(m) \neq \emptyset$, para todo $n \in \omega$.

Definição 2.1.1. Sejam $f \in \mathcal{F}$ e $i \in\{1,2\}$. Dizemos que $f$ é do tipo $i$ se $f$ satisfaz a condição (i) descrita acima.

Proposição 2.1.2. Se $f: \omega \rightarrow \mathbb{Z}^{(\mathfrak{c})}$, então existe $j: \omega \rightarrow \omega$ estritamente crescente satisfazendo uma das seguintes condições:

(i) $f \circ j$ é do tipo 1;

(ii) $f \circ j=f_{1}+f_{2}$, onde $f_{1}, f_{2}: \omega \rightarrow \mathbb{Z}^{(\mathfrak{c})}$, $f_{2}$ é do tipo 2 ou constante e existe $m$ um número natural tal que, para cada $n \in \omega, f_{1}(n)$ é uma combinação linear com coeficientes pertencentes $a\{-1,1\}$ de $m$ elementos de $\left\{\chi_{n}: n \in \omega\right\} .^{2}$

Demonstração. Há dois casos a serem considerados:

\footnotetext{
${ }^{2}$ Se $m=0$, então $f_{1}(n)=0$, para todo $n \in \omega$.
} 
Caso 1: $\{\|f(n)\|: n \in \omega\}$ é ilimitado.

Por indução, tomemos $\left\{n_{k}: k \in \omega\right\}$ uma seqüência estritamente crescente de números naturais tal que $\left\|f\left(n_{k}\right)\right\|>k$, para todo $k \in \omega$. Temos que

$$
\begin{aligned}
j: \omega & \rightarrow \omega \\
k & \mapsto n_{k}
\end{aligned}
$$

é uma função estritamente crescente tal que $f \circ j$ é do tipo 1.

Caso 2: $\{\|f(n)\|: n \in \omega\}$ é limitado.

Sejam $\tilde{f}_{1}, \tilde{f}_{2}: \omega \rightarrow \mathbb{Z}^{(\mathfrak{c})}$ dadas por

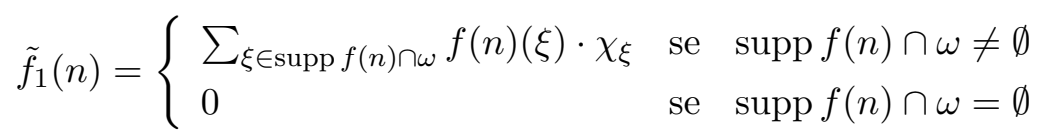

$\mathrm{e}$

$$
\tilde{f}_{2}(n)=\left\{\begin{array}{lll}
\sum_{\xi \in \operatorname{supp} f(n) \backslash \omega} f(n)(\xi) \cdot \chi_{\xi} & \text { se } & \operatorname{supp} f(n) \backslash \omega \neq \emptyset \\
0 & \text { se } & \operatorname{supp} f(n) \backslash \omega=\emptyset .
\end{array}\right.
$$

Observamos que $f(n)=\tilde{f}_{1}(n)+\tilde{f}_{2}(n)$, para todo $n \in \omega$.

Se $\cup_{n \in \omega} \operatorname{supp} \tilde{f}_{2}(n)$ for infinito, então existe $j_{1}: \omega \rightarrow \omega$ estritamente crescente tal que $\tilde{f}_{2} \circ j_{1}$ é do tipo 2. Se $\cup_{n \in \omega} \operatorname{supp} \tilde{f}_{2}(n)$ for finito, então existe $j_{1}: \omega \rightarrow \omega$ estritamente crescente tal que $\tilde{f}_{2} \circ j_{1}$ é constante. ${ }^{3}$

Temos que $\left\{\left\|\tilde{f}_{1} \circ j_{1}(n)\right\|: n \in \omega\right\}$ é limitado e, portanto, existem $m \in \omega$ e $j_{2}: \omega \rightarrow \omega$ estritamente crescente tais que $\left\|\tilde{f}_{1} \circ j_{1} \circ j_{2}(n)\right\|=m$, qualquer que seja $n \in \omega$. Sejam $j=j_{1} \circ j_{2}, f_{1}=\tilde{f}_{1} \circ j$ e $f_{2}=\tilde{f}_{2} \circ j$. Note que $f_{1}, f_{2}: \omega \rightarrow \mathbb{Z}^{(\mathfrak{c})}, f \circ j=f_{1}+f_{2}$ e $f_{2}$ é do tipo 2 ou constante.

Se $m=0$, então $f_{1}(n)=0$, para todo $n \in \omega$. Se $m>0$, então $\operatorname{supp} f_{1}(n) \neq \emptyset$, qualquer que seja $n \in \omega$. Para cada $n \in \omega$, consideremos $\left\{\xi_{0}^{n}, \ldots, \xi_{k_{n}}^{n}\right\}$ uma indexação de $\operatorname{supp} f_{1}(n)$

\footnotetext{
${ }^{3}$ De fato, temos que $\cup_{n \in \omega} \operatorname{supp} \tilde{f}_{2}(n)=\cup_{n \in \omega}(\operatorname{supp} f(n) \backslash \omega)$ e, portanto, $\left(\cup_{n \in \omega} \operatorname{supp} \tilde{f}_{2}(n)\right) \cap \omega=\emptyset$. Suponhamos que $\cup_{n \in \omega} \operatorname{supp} \tilde{f}_{2}(n)$ seja infinito. Façamos $n_{0}=0$ e suponhamos definidos $n_{0}, n_{1}, \ldots, n_{k}$ números naturais. Tomemos $n_{k+1}>n_{k}$ tal que $\operatorname{supp} \tilde{f}_{2}\left(n_{k+1}\right) \backslash \cup_{l<k+1} \operatorname{supp} \tilde{f}_{2}\left(n_{l}\right) \neq \emptyset$. Temos que $j_{1}: \omega \rightarrow \omega$ dada por $j(k)=n_{k}$ é estritamente crescente e $\tilde{f}_{2} \circ j_{1}$ é do tipo 2. Suponhamos, agora, que $\cup_{n \in \omega} \operatorname{supp} \tilde{f}_{2}(n)$ seja finito. Como $\{|| f(n) \|: n \in \omega\}$ é limitado, $\left\{\left|\tilde{f}_{2}(n)\right|: n \in \omega\right\}$ também o é. Logo, $\left\{\tilde{f}_{2}(n): n \in \omega\right\}$ é um conjunto finito e, portanto, existe $j_{1}: \omega \rightarrow \omega$ estritamente crescente tal que $\tilde{f}_{2} \circ j_{1}$ é constante.
} 
tal que $\xi_{0}^{n}<\xi_{1}^{n}<\ldots<\xi_{k_{n}}^{n}$. Temos que

$$
f_{1}(n)=\sum_{i=0}^{k_{n}} \operatorname{sgn}\left(f_{1}(n)\left(\xi_{i}^{n}\right)\right) \cdot(\underbrace{\chi_{\xi_{i}^{n}}+\ldots+\chi_{i}^{n}}_{\left|f_{1}(n)\left(\xi_{i}^{n}\right)\right| \text { vezes }})
$$

onde sgn : $\mathbb{Z} \rightarrow\{-1,1\}$ é dada por

$$
\operatorname{sgn}(a)=\left\{\begin{array}{rll}
1 & \text { se } & a \geq 0 \\
-1 & \text { se } & a<0 .
\end{array}\right.
$$

Como

$$
\sum_{i=0}^{k_{n}}\left|f_{1}(n)\left(\xi_{i}^{n}\right)\right|=\left\|f_{1}(n)\right\|=m
$$

temos que $f_{1}(n)$ se escreve como combinação linear de $m$ elementos de $\left\{\chi_{n}: n \in \omega\right\}$ com coeficientes pertencentes a $\{-1,1\}$.

Proposição 2.1.3. Existe $\left\{f_{\xi}: \omega \leq \xi<\mathfrak{c}\right\}$ uma indexação de $\mathcal{F}$ tal que $\cup_{n \in \omega} \operatorname{supp} f_{\xi}(n) \subset \xi$, para todo $\xi \in[\omega, \mathfrak{c}[$.

Demonstração. Observamos, primeiramente, que $|\mathcal{F}|=\mathfrak{c}^{\omega}=\mathfrak{c}$. Portanto, podemos considerar $\left\{\tilde{f}_{\xi}: \omega \leq \xi<\mathfrak{c}\right\}$ uma indexação de $\mathcal{F}$ tal que, para cada $f \in \mathcal{F}$, o conjunto

$$
A_{f}=\left\{\xi \in \left[\omega, \mathfrak{c}\left[: f=\tilde{f}_{\xi}\right\}\right.\right.
$$

tenha cardinalidade $\boldsymbol{c}^{4}$

Fixemos $f \in \mathcal{F}$. Como $\cup_{n \in \omega} \operatorname{supp} f(n) \subset \mathfrak{c}, \operatorname{cf}(\mathfrak{c})>\omega$ e $\left|\cup_{n \in \omega} \operatorname{supp} f(n)\right| \leq \omega$, existe $\alpha<\mathfrak{c}$ tal que $\cup_{n \in \omega} \operatorname{supp} f(n) \subset \alpha$. Seja $\xi \in A_{f}$. Se $\xi<\alpha$, façamos $f_{\xi}=g$, onde $g(n)=(n+1) \cdot \chi_{0}$, para todo $n \in \omega$. Temos que $g$ é do tipo 1 e, portanto, $g \in \mathcal{F}$. Se $\xi \geq \alpha$, façamos $f_{\xi}=\tilde{f}_{\xi}$.

Vê-se facilmente $\left\{f_{\xi}: \omega \leq \xi<\mathfrak{c}\right\}$ é uma indexação de $\mathcal{F}$ tal que $\cup_{n \in \omega} \operatorname{supp} f_{\xi}(n) \subset \xi$, para todo $\xi \in[\omega, \mathfrak{c}[$.

\footnotetext{
${ }^{4}$ Seja $\phi: \mathfrak{c} \times \mathfrak{c} \rightarrow \mathfrak{c} \backslash \omega$ uma bijeção. Escrevamos $\mathfrak{c} \backslash \omega=\cup_{\xi<\mathfrak{c}} \phi(\{\xi\} \times \mathfrak{c})$. Para cada $\xi<\mathfrak{c}$, consideremos $\left\{\tilde{f}_{\mu}: \mu \in \phi(\{\xi\} \times \mathfrak{c})\right\}$ uma indexação de $\mathcal{F}$. Temos que $\left\{\tilde{f}_{\mu}: \mu \in \cup_{\xi<\mathfrak{c}} \phi(\{\xi\} \times \mathfrak{c})\right\}=\left\{\tilde{f}_{\mu}: \omega \leq \mu<\mathfrak{c}\right\}$ tem a propriedade desejada.
} 


\subsection{Construindo homomorfismos de grupos}

Sejam $\left\{p_{\xi}: \omega \leq \xi<\mathfrak{c}\right\}$ uma família de ultrafiltros seletivos dois a dois incomparáveis segundo a ordem de Rudin-Keisler e $\left\{f_{\xi}: \omega \leq \xi<\mathfrak{c}\right\}$ uma indexação de $\mathcal{F}$ tal que $\cup_{n \in \omega} \operatorname{supp} f_{\xi}(n) \subset \xi$, qualquer que seja $\xi \in\left[\omega, \mathfrak{c}\left[\right.\right.$. Para cada $J \in \mathbb{Z}^{(\mathfrak{c})} \backslash\{0\}$, construiremos $\phi_{J}: \mathbb{Z}^{(\mathfrak{c})} \rightarrow \mathbb{T}$ um homomorfismo de grupos tal que:

- $\phi_{J}(J) \neq 0+\mathbb{Z}$

- $\phi_{J}\left(\chi_{\xi}\right)=p_{\xi}-\lim \left\{\phi_{J}\left(f_{\xi}(n)\right): n \in \omega\right\}$, para todo $\xi \in[\omega, \mathfrak{c}[$

- $\phi_{J}\left(\chi_{n}\right) \rightarrow 0+\mathbb{Z}$.

A fim de conciliar a primeira condição com as demais, começaremos definindo $\phi_{J}$ em $\mathbb{Z}^{(E)}$, onde $E \in[\mathfrak{c}]^{\omega}$ é dado pela proposição abaixo.

Proposição 2.2.1. Seja $J \in \mathbb{Z}^{(\mathfrak{c})} \backslash\{0\}$. Existe $E \in[\mathfrak{c}]^{\omega}$ tal que:

(i) $\operatorname{supp} J \subset E$;

(ii) $|E \backslash \omega|=\omega$;

(iii) $\cup_{n \in \omega} \operatorname{supp} f_{\xi}(n) \subset E$, qualquer que seja $\xi \in E \backslash \omega$.

Demonstração. Para cada $n \in \omega$, façamos $E(n)=\omega$. Se $\xi \in[\omega, \mathfrak{c}[$, definimos indutivamente

$$
E(\xi)=\{\xi\} \cup \bigcup_{\mu \in \cup_{n \in \omega} \operatorname{supp} f_{\xi}(n)} E(\mu)
$$

e façamos

$$
E=\bigcup_{\zeta \in \operatorname{supp} J \cup[\omega, \omega+\omega[} E(\zeta) .
$$

Temos que supp $J \cup[\omega, \omega+\omega[\subset E$, uma vez que $\zeta \in E(\zeta) \subset E$, qualquer que seja $\zeta \in$ $\operatorname{supp} J \cup\left[\omega, \omega+\omega\left[\right.\right.$. Além disso, um argumento indutivo garante que $E(\xi) \in[\mathfrak{c}]^{\omega}$ para todo $\xi<\mathfrak{c}$ e, portanto, $E \in[\mathfrak{c}]^{\omega}$. Por fim, se $\xi \in E \backslash \omega$, então existe $\zeta \in \operatorname{supp} J \cup[\omega, \omega+\omega[$ tal que $\xi \in E(\zeta)$. Um outro argumento indutivo garante que se $\alpha \in E(\beta)$, então $E(\alpha) \subset E(\beta)$, quaisquer que sejam $\alpha, \beta<\mathfrak{c}{ }^{5} \operatorname{Logo}, \cup_{n \in \omega} \operatorname{supp} f_{\xi}(n) \subset E(\xi) \subset E(\zeta) \subset E$.

\footnotetext{
${ }^{5}$ Temos que $E(n)=\omega$, para todo $n \in \omega$. Logo, se $n \in \omega$ e $m \in E(n)$, então $E(m)=E(n)$. Fixemos $\beta \in[\omega, \mathfrak{c}[$ e suponhamos que, para todo $\gamma<\beta$, valha a seguinte propriedade: se $\delta \in E(\gamma)$, então $E(\delta) \subset E(\gamma)$. Tomemos $\alpha \in E(\beta)$. Se $\alpha=\beta$, então $E(\alpha)=E(\beta)$. Suponhamos, portanto, $\alpha \neq \beta$. Temos que $\alpha \in E(\mu) \subset E(\beta)$, para algum $\mu \in \cup_{n \in \omega} \operatorname{supp} f_{\beta}(n)$. Como $\cup_{n \in \omega} \operatorname{supp} f_{\beta}(n) \subset \beta$, segue que $\mu<\beta$. Por hipótese de indução, $E(\alpha) \subset E(\mu)$. Logo, $E(\alpha) \subset E(\beta)$.
} 
Lema 2.2.2. Sejam $J \in \mathbb{Z}^{(\mathfrak{c})} \backslash\{0\}$ e $E \in[\mathfrak{c}]^{\omega}$ satisfazendo as condições (i), (ii) e (iii) $d a$ proposição 2.2.1. Existe $\left\{E_{k}: k \in \omega\right\}$ uma familia de subconjuntos finitos de $E,\left\{b_{k}: k \in \omega\right\}$ uma seqüência estritamente crescente de números naturais, $\left\{r_{k}: k \in \omega\right\}$ uma seqüência de números reais positivos e $i: \omega \rightarrow E \backslash \omega$ tais que:

(i) $\operatorname{supp} J \subset E_{0}$;

(ii) $E=\cup_{k \in \omega} E_{k}$;

(iii) $i(k) \in E_{k}$, para todo $k \in \omega$;

(iv) $E_{k+1} \supset E_{k} \cup \bigcup\left\{\operatorname{supp} f_{i(m)}\left(b_{m}\right): m \leq k\right\}$, para todo $k \in \omega$;

(v) $\left\{b_{k}: k \in i^{-1}(\{\xi\})\right\} \in p_{\xi}$, para todo $\xi \in E \backslash \omega$;

(vi) Se $f_{i(k)}$ é do tipo 1, então $\left\|f_{i(k)}\left(b_{k}\right)\right\| \cdot r_{k}>4$;

(vii) Se $f_{i(k)}$ é do tipo 2, então $\operatorname{supp} f_{i(k)}\left(b_{k}\right) \backslash E_{k} \neq \emptyset$;

(viii) $r_{0}=\frac{1}{4 \cdot\|J\|}$;

(ix) $r_{k+1}=\frac{r_{k}}{2 \cdot\left\|f_{i(k)}\left(b_{k}\right)\right\|}$, para todo $k \in \omega$.

Demonstração. Consideremos $\left\{a_{n}: n \in \omega\right\}$ uma enumeração de $E$ tal que $a_{0} \notin \omega$. Sejam $F_{0}=\operatorname{supp} J \cup\left\{a_{0}\right\}$ e $F_{n+1}=F_{n} \cup \bigcup\left\{\operatorname{supp} f_{\xi}(m): m \leq n, \xi \in F_{n} \backslash \omega\right\} \cup\left\{a_{n+1}\right\}$, para cada $n \in \omega$. Temos que $\left\{F_{n}: n \in \omega\right\}$ é uma família de subconjuntos finitos de $E$ tal que:

(1) $\operatorname{supp} J \subset F_{0}$;

(2) $E=\cup_{n \in \omega} F_{n}$;

(3) $F_{n+1} \supset F_{n} \cup \bigcup\left\{\operatorname{supp} f_{\xi}(m): m \leq n, \xi \in F_{n} \backslash \omega\right\}$.

Fixemos $\xi \in E \backslash \omega$ e $n \in \omega$. Se $f_{\xi}$ é do tipo 1, definimos

$$
A_{n}^{\xi}=\left\{k \in \omega:\left\|f_{\xi}(k)\right\|>2^{n+4} \cdot Y_{n-1}\right\}
$$

onde

$$
Y_{m}=\|J\| \cdot \prod_{\substack{l \leq m \\ \zeta \in \bar{F}_{m} \backslash \omega}}\left\|f_{\zeta}(l)\right\|
$$


para todo $m \in \omega \mathrm{e}$

$$
Y_{-1}=\|J\|
$$

Se $f_{\xi}$ é do tipo 2 , definimos

$$
A_{n}^{\xi}=\left\{k \in \omega: \operatorname{supp} f_{\xi}(k) \backslash F_{n} \neq \emptyset\right\}
$$

Afirmamos que $A_{n}^{\xi}$ é um subconjunto cofinito de $\omega$, quaisquer que sejam $n \in \omega$ e $\xi \in E \backslash \omega$. De fato, sejam $\xi \in E \backslash \omega$ e $n \in \omega$. Se $f_{\xi}$ é do tipo 1 , então $A_{n}^{\xi} \supset\left\{k \in \omega: k \geq 2^{n+4} \cdot Y_{n-1}\right\}$ e, portanto, $A_{n}^{\xi}$ é um subconjunto cofinito de $\omega$. Suponhamos que $f_{\xi}$ seja do tipo 2 . Se $A_{n}^{\xi}$ não fosse um subconjunto cofinito de $\omega$, então $\omega \backslash A_{n}^{\xi}$ seria um conjunto infinito. Como $\omega \backslash A_{n}^{\xi}=\left\{k \in \omega: \operatorname{supp} f_{\xi}(k) \subset F_{n}\right\}$, teríamos que $\cup_{k \in \omega \backslash A_{n}^{\xi}} \operatorname{supp} f_{\xi}(k) \subset F_{n}$, o que é absurdo, uma vez que $f_{\xi}$ é do tipo 2 e $F_{n}$ é finito.

Logo, para cada $\xi \in E \backslash \omega$, temos que $\left\{A_{n}^{\xi}: n \in \omega\right\} \subset p_{\xi}$, uma vez que $p_{\xi}$ é um ultrafiltro livre sobre $\omega$. Da seletividade de $p_{\xi}$ decorre que existe uma seqüência $\left\{a_{n}^{\xi}: n \in \omega\right\} \in p_{\xi}$ tal que $a_{n}^{\xi} \in A_{n}^{\xi}$ e $n<a_{n}^{\xi}$, para cada $n \in \omega$. Com efeito, fixemos $\xi \in E \backslash \omega$. Para cada $n \in \omega$, seja $B_{n}^{\xi}=A_{n}^{\xi} \backslash\{m \in \omega: m \leq n\}$. Como $A_{n}^{\xi} \in p_{\xi}$ e $p_{\xi}$ é um ultrafiltro livre sobre $\omega$, temos que $B_{n}^{\xi} \in p_{\xi}$. Do fato de $p_{\xi}$ ser seletivo decorre que existe $B \in p_{\xi}$ tal que $B \subset^{*} B_{n}^{\xi}$, para todo $n \in \omega$. Tomemos $\left\{c_{n}: n \in \omega\right\}$ uma seqüência estritamente crescente de números naturais tal que $B \backslash\left\{m \in \omega: m<c_{n}\right\} \subset B_{n}^{\xi}$ e $\left.] c_{n}, c_{n+1}\right] \cap B \neq \emptyset$. Para cada $n \in \omega$, consideremos $\left.\left.D_{n}=\right] c_{n}, c_{n+1}\right] \cap B$. Temos que $\left\{D_{n}: n \in \omega\right\} \cup\left\{\omega \backslash \cup_{n \in \omega} D_{n}\right\}$ é uma partição de $\omega$ tal que $\omega \backslash \cup_{n \in \omega} D_{n} \notin p_{\xi}$ e $D_{n} \notin p_{\xi}$, para todo $n \in \omega$. Da seletividade de $p_{\xi}$ segue que existe $\left\{a_{n}^{\xi}: n \in \omega\right\} \in p_{\xi}$ tal que $a_{n}^{\xi} \in D_{n}$, para todo $n \in \omega$. Em particular, $a_{n} \in B_{n}^{\xi}$, o que implica que $a_{n}^{\xi} \in A_{n}^{\xi}$ e $n<a_{n}^{\xi}$, para todo $n \in \omega$.

Do lema 1.5.4 segue que existe $\left\{I_{\xi}: \xi \in E \backslash \omega\right\}$ uma família de subconjuntos de $\omega$ dois a dois disjuntos tal que:

(a) $\left\{a_{n}^{\xi}: n \in I_{\xi}\right\} \in p_{\xi}$, para cada $\xi \in E \backslash \omega$;

(b) $\left\{\left[n, a_{n}^{\xi}\right]: n \in I_{\xi}, \xi \in E \backslash \omega\right\}$ é uma família de intervalos de $\omega$ dois a dois disjuntos.

Para cada $\xi \in E \backslash \omega$, seja

(c) $N_{\xi}=\min \left\{n \in \omega: \xi \in F_{n}\right\}$.

Podemos supor, sem perda de generalidade, que 
(d) $N_{\xi}<n$, para todo $n \in I_{\xi} \cdot 6$

Consideremos $\left\{n_{k}: k \in \omega\right\}$ uma enumeração estritamente crescente de $\dot{\cup}_{\xi \in E \backslash \omega} I_{\xi} \cdot{ }^{7}$ Seja

$$
\begin{aligned}
i: \omega & \rightarrow E \backslash \omega \\
k & \mapsto i(k)
\end{aligned}
$$

onde $i(k)$ é o único elemento de $E \backslash \omega$ tal que $n_{k} \in I_{i(k)}$.

Para cada $k \in \omega$, façamos $b_{k}=a_{n_{k}}^{i(k)}, E_{k}=F_{n_{k}}$ e definamos $r_{k}$ segundo os ítens (viii) e (ix) do enunciado deste lema. Resta mostrar que as condições (i)-(vii) estão satisfeitas.

(i) Como $0 \leq n_{0}$, temos que $E_{0}=F_{n_{0}} \supset F_{0} \supset \operatorname{supp} J$.

(ii) $\cup_{k \in \omega} E_{k}=\cup_{k \in \omega} F_{n_{k}}=E$.

(iii) Seja $k \in \omega$. Temos que $n_{k} \in I_{i(k)}$. De (d) segue que $N_{i(k)}<n_{k}$. De (c) e (3) vem que $i(k) \in F_{N_{i(k)}} \subset F_{n_{k}}=E_{k}$.

(iv) De (3) vem que $E_{k} \subset E_{k+1}$, pois $\left\{n_{k}: k \in \omega\right\}$ é uma enumeração estritamente crescente de $\dot{U}_{\xi \in E \backslash \omega} I_{\xi}$. Resta mostrar que supp $f_{i(m)}\left(b_{m}\right) \subset E_{k+1}$, para cada $m \leq k$. De (3) decorre que

$$
E_{k+1}=F_{n_{k+1}} \supset \bigcup\left\{\operatorname{supp} f_{\xi}(m): m \leq n_{k+1}-1, \xi \in F_{n_{k+1}-1} \backslash \omega\right\} .
$$

Por (iii), $i(m) \in E_{m} \subset E_{k}=F_{n_{k}} \subset F_{n_{k+1}-1}$, para todo $m \leq k$. Além disso, $i(m) \notin \omega$. Por (b), $b_{m}=a_{n_{m}}^{i(m)} \leq n_{k+1}-1$, para todo $m \leq k$. Logo, $\operatorname{supp} f_{i(m)}\left(b_{m}\right) \subset E_{k+1}$.

(v) Seja $\xi \in E \backslash \omega$. Temos que $\left\{b_{k}: k \in i^{-1}(\{\xi\})\right\}=\left\{a_{n_{k}}^{i(k)}: k \in i^{-1}(\{\xi\})\right\}=\left\{a_{n}^{\xi}: n \in I_{\xi}\right\} \in p_{\xi}$, por (a).

(vi) Suponhamos que $f_{i(k)}$ seja do tipo 1. Temos que $b_{k}=a_{n_{k}}^{i(k)} \in A_{n_{k}}^{i(k)}$. Da definição de $A_{n_{k}}^{i(k)}$ vem que

$$
\left\|f_{i(k)}\left(b_{k}\right)\right\|>2^{n_{k}+4} \cdot Y_{n_{k}-1}
$$

onde

$$
Y_{n_{k}-1}=\|J\| \cdot \prod_{\substack{l \leq n_{k}-1 \\ \zeta \in \bar{F}_{n_{k}-1} \backslash \omega}}\left\|f_{\zeta}(l)\right\| .
$$

\footnotetext{
${ }^{6}$ As condições (a) e (b) permanecem válidas se substituirmos $I_{\xi}$ por $I_{\xi} \backslash\left\{0,1, \ldots, N_{\xi}\right\}$.

${ }^{7}$ Logo, $k \leq n_{k}$, para cada $k \in \omega$.
} 
Observamos que se $m<k$, então $n_{m} \leq n_{k-1}$ e, portanto, $i(m) \in E_{m}=F_{n_{m}} \subset F_{n_{k-1}} \subset$ $F_{n_{k}-1}$. Note que $i(m) \notin \omega$. Como $b_{m}=a_{n_{m}}^{i(m)}$ e $\left[n_{m}, a_{n_{m}}^{i(m)}\right] \cap\left[n_{k}, a_{n_{k}}^{i(k)}\right]=\emptyset$, temos que $b_{m}<n_{k}$ e, portanto, $b_{m} \leq n_{k}-1$. Uma vez que

$$
r_{k}=\frac{1}{2^{k+2} \cdot\|J\| \cdot \prod_{m<k}\left\|f_{i(m)}\left(b_{m}\right)\right\|}
$$

concluímos que

$$
\left\|f_{i(k)}\left(b_{k}\right)\right\| \cdot r_{k}>4
$$

(vii) Suponhamos que $f_{i(k)}$ seja do tipo 2. Temos que $b_{k}=a_{n_{k}}^{i(k)} \in A_{n_{k}}^{i(k)}$. Da definição de $A_{n_{k}}^{i(k)}$ vem que

$$
\operatorname{supp} f_{i(k)}\left(b_{k}\right) \backslash E_{k}=\operatorname{supp} f_{i(k)}\left(b_{k}\right) \backslash F_{n_{k}} \neq \emptyset \text {. }
$$

Recordamos que $\mathcal{B}$ denota o conjunto de todos os arcos abertos não vazios de $\mathbb{T}$.

Lema 2.2.3. Sejam $J \in \mathbb{Z}^{(\mathfrak{c})} \backslash\{0\}$ e $E \in[\mathfrak{c}]^{\omega}$ satisfazendo as condições (i), (ii) e (iii) $d a$ proposição 2.2.1. Existe $\phi_{J} \uparrow_{\mathbb{Z}^{(E)}}: \mathbb{Z}^{(E)} \rightarrow \mathbb{T}$ um homomorfismo de grupos com as seguintes propriedades:

(i) $\phi_{J} \uparrow_{\mathbb{Z}^{(E)}}(J) \neq 0+\mathbb{Z}$;

(ii) $\phi_{J} \Upsilon_{\mathbb{Z}^{(E)}}\left(\chi_{\xi}\right)=p_{\xi}-\lim \left\{\phi_{J} \Upsilon_{\mathbb{Z}^{(E)}}\left(f_{\xi}(n)\right): n \in \omega\right\}$, para cada $\xi \in E \backslash \omega$;

(iii) $\phi_{J} \Upsilon_{\mathbb{Z}^{(E)}}\left(\chi_{n}\right) \rightarrow 0+\mathbb{Z}^{8}$

Demonstração. Consideremos $\left\{E_{k}: k \in \omega\right\},\left\{b_{k}: k \in \omega\right\},\left\{r_{k}: k \in \omega\right\}$ e $i: \omega \rightarrow E \backslash \omega$ de acordo com o lema 2.2.2. A fim de exibirmos um homomorfismo de grupos $\phi_{J} \Upsilon_{\mathbb{Z}^{(E)}}$ satisfazendo as condições (i), (ii) e (iii) deste lema, construiremos indutivamente aplicações auxiliares $\psi_{k}: E \rightarrow \mathcal{B}$, para cada $k \in \omega$.

Para cada $\xi \in E_{0}$, tomemos $y_{\xi} \in \mathbb{R}$ tal que

$$
\sum_{\xi \in \operatorname{supp}} J(\xi) \cdot y_{\xi}=\frac{1}{2}
$$

e definamos $\psi_{0}(\xi)$ como sendo o arco aberto de $\mathbb{T}$ centrado em $y_{\xi}+\mathbb{Z}$ com diâmetro $r_{0}$. Temos

${ }^{8}$ Estamos considerando $\mathbb{Z}^{(E)} \subset \mathbb{Z}^{(\mathfrak{c})}$. 
que

$$
\delta\left(\sum_{\xi \in \operatorname{supp} J} J(\xi) \cdot \psi_{0}(\xi)\right) \leq \sum_{\xi \in \operatorname{supp} J}|J(\xi)| \cdot \delta\left(\psi_{0}(\xi)\right)=\|J\| \cdot r_{0}=\frac{1}{4}
$$

Logo,

$$
0+\mathbb{Z} \notin \sum_{\xi \in \operatorname{supp} J} J(\xi) \cdot \psi_{0}(\xi)
$$

uma vez que

$$
\frac{1}{2}+\mathbb{Z} \in \sum_{\xi \in \operatorname{supp} J} J(\xi) \cdot \psi_{0}(\xi)
$$

Por fim, se $\xi \in E \backslash E_{0}$, façamos $\psi_{0}(\xi)=\mathbb{T}$.

Seja $k \in \omega$ e suponhamos definida $\psi_{k}: E \rightarrow \mathcal{B}$. Vamos construir $\psi_{k+1}: E \rightarrow \mathcal{B}$ com as seguintes propriedades:

(1) $\overline{\psi_{k+1}(\xi)} \subset \psi_{k}(\xi)$, para todo $\xi \in E$;

(2) Se $\xi \in E \backslash E_{k+1}$, então $\psi_{k+1}(\xi)=\mathbb{T}$;

Se $\xi \in E_{k+1}$, então $\delta\left(\psi_{k+1}(\xi)\right)=r_{k+1}$;

(3) $\psi_{k}(i(k)) \cap \sum_{\mu \in \operatorname{supp} f_{i(k)}\left(b_{k}\right)} f_{i(k)}\left(b_{k}\right)(\mu) \cdot \psi_{k+1}(\mu) \neq \emptyset$;

(4) Se $\xi \in\left(E_{k+1} \backslash E_{k}\right) \cap \omega$, então $\delta(x, 0+\mathbb{Z})<r_{k}$, para todo $x \in \psi_{k+1}(\xi)$.

Se $\xi \in\left(E_{k+1} \backslash E_{k}\right) \cap \omega$, seja $\tilde{\psi}_{k}(\xi)$ o arco aberto de $\mathbb{T}$ centrado em $0+\mathbb{Z}$ com diâmetro $r_{k}$. Se $\xi \in\left(E_{k+1} \backslash E_{k}\right) \backslash \omega$, seja $\tilde{\psi}_{k}(\xi)$ um arco aberto de $\mathbb{T}$ com diâmetro $r_{k}$. Se $\xi \in E_{k}$, tomemos $\tilde{\psi}_{k}(\xi)=\psi_{k}(\xi)$.

Se $\xi \in E \backslash E_{k+1}$, façamos $\psi_{k+1}(\xi)=\mathbb{T}$. Se $\xi \in E_{k+1} \backslash \operatorname{supp} f_{i(k)}\left(b_{k}\right)$, seja $\psi_{k+1}(\xi)$ o arco aberto de $\mathbb{T}$ centrado no ponto médio de $\tilde{\psi}_{k}(\xi)$ com diâmetro $r_{k+1}$. Vamos, agora, definir $\psi_{k+1}(\xi)$ para $\xi \in \operatorname{supp} f_{i(k)}\left(b_{k}\right)$.

Caso 1: $f_{i(k)}$ é do tipo 1.

Temos que

$$
\sum_{\mu \in \operatorname{supp} f_{i(k)}\left(b_{k}\right)} f_{i(k)}\left(b_{k}\right)(\mu) \cdot \tilde{\tilde{\psi}}_{k}(\mu)=\mathbb{T}
$$

onde $\tilde{\tilde{\psi}}_{k}(\mu)$ é o arco aberto de $\mathbb{T}$ centrado no ponto médio de $\tilde{\psi}_{k}(\mu)$ com diâmetro

$$
\delta\left(\tilde{\tilde{\psi}}_{k}(\mu)\right)=\frac{r_{k}}{4} .
$$


Portanto, para cada $\mu \in \operatorname{supp} f_{i(k)}\left(b_{k}\right)$, existe $x_{\mu}^{k} \in \tilde{\tilde{\psi}}_{k}(\mu)$ tal que

$$
\sum_{\mu \in \operatorname{supp} f_{i(k)}\left(b_{k}\right)} f_{i(k)}\left(b_{k}\right)(\mu) \cdot x_{\mu}^{k} \in \psi_{k}(i(k))
$$

Consideremos $\psi_{k+1}(\mu)$ o arco aberto de $\mathbb{T}$ centrado em $x_{\mu}^{k}$ com diâmetro $r_{k+1}$.

As condições $(1),(2),(3)$ e (4) estão verificadas. De fato, se $\xi \in E_{k}$, então $\tilde{\tilde{\psi}}_{k}(\xi)$ é o arco aberto de $\mathbb{T}$ centrado no ponto médio de $\psi_{k}(\xi)$ com diâmetro $\frac{r_{k}}{4}$. Como $x_{\xi}^{k} \in \tilde{\psi}_{k}(\xi)$ e $r_{k+1} \leq \frac{r_{k}}{2}$, temos que $\overline{\psi_{k+1}(\xi)} \subset \psi_{k}(\xi)$. Se $\xi \in\left(E_{k+1} \backslash E_{k}\right) \cap \omega$, então $\tilde{\tilde{\psi}}_{k}(\xi)$ é o arco aberto de $\mathbb{T}$ centrado em $0+\mathbb{Z}$ com diâmetro $\frac{r_{k}}{4}$. Como $x_{\xi}^{k} \in \tilde{\tilde{\psi}}_{k}(\xi)$ e $r_{k+1} \leq \frac{r_{k}}{2}$, temos que $\delta(x, 0+\mathbb{Z})<r_{k}$, para todo $x \in \psi_{k+1}(\xi)$.

Caso 2: $f_{i(k)}$ é do tipo 2 .

Tomemos $\alpha \in \operatorname{supp} f_{i(k)}\left(b_{k}\right) \backslash E_{k}$. Para cada $\xi \in \operatorname{supp} f_{i(k)}\left(b_{k}\right) \backslash\{\alpha\}$, denotemos por $z_{\xi}$ o ponto médio de $\tilde{\psi}_{k}(\xi)$. Como $\psi_{k}(\alpha)=\mathbb{T}$, existe $z_{\alpha} \in \psi_{k}(\alpha)$ tal que

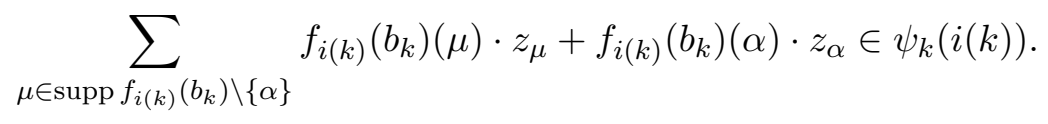

Assim, para cada $\xi \in \operatorname{supp} f_{i(k)}\left(b_{k}\right)$, definimos $\psi_{k+1}(\xi)$ como sendo o arco aberto de $\mathbb{T}$ centrado em $z_{\xi}$ com diâmetro $r_{k+1}$.

As condições (1), (2), (3) e (4) estão verificadas, uma vez que supp $f_{i(k)}\left(b_{k}\right) \cap \omega=\emptyset$.

Como $\mathbb{T}$ é um espaço métrico completo e $\left(r_{k}\right)_{k \in \omega}$ é uma seqüência de números reais positivos que converge para 0 , concluímos que se $\xi \in E$, então $\cap_{k \in \omega} \psi_{k}(\xi)=\cap_{k \in \omega} \overline{\psi_{k}(\xi)}$ é um conjunto unitário. Denotaremos por $\phi\left(\chi_{\xi}\right)$ o único elemento de $\cap_{k \in \omega} \psi_{k}(\xi)$. Uma vez que $\left\{\chi_{\xi}: \xi \in E\right\}$ é um subconjunto independente e gerador de $\mathbb{Z}^{(E)}$ é possível estender $\phi$ a um homomorfismo de $\operatorname{grupos} \phi_{J} \uparrow_{\mathbb{Z}^{(E)}}: \mathbb{Z}^{(E)} \rightarrow \mathbb{T}$.

Temos que

$$
\phi_{J} \Upsilon_{\mathbb{Z}^{(E)}}(J)=\sum_{\mu \in \operatorname{supp} J} J(\mu) \cdot \phi_{J} \Upsilon_{\mathbb{Z}^{(E)}}\left(\chi_{\mu}\right) \in \sum_{\mu \in \operatorname{supp} J} J(\mu) \cdot \psi_{0}(\mu)
$$

e, portanto, $\phi_{J} \uparrow_{\mathbb{Z}^{(E)}}(J) \neq 0+\mathbb{Z}$. Logo, (i) está verificada. 
Fixemos $\xi \in E \backslash \omega$. Para cada $k \in i^{-1}(\{\xi\})$, temos que

$$
\phi_{J} \uparrow_{\mathbb{Z}^{(E)}}\left(f_{i(k)}\left(b_{k}\right)\right) \in \sum_{\mu \in \operatorname{supp} f_{i(k)}\left(b_{k}\right)} f_{i(k)}\left(b_{k}\right)(\mu) \cdot \psi_{k+1}(\mu)
$$

e

$$
\phi_{J}\left\lceil_{\mathbb{Z}^{(E)}}\left(\chi_{i(k)}\right) \in \psi_{k}(i(k)) .\right.
$$

De (3) segue que

$$
\begin{aligned}
\delta\left(\phi_{J} \Upsilon_{\mathbb{Z}^{(E)}}\left(f_{i(k)}\left(b_{k}\right)\right), \phi_{J}\left\lceil_{\mathbb{Z}^{(E)}}\left(\chi_{i(k)}\right)\right)\right. & \leq d_{1}+d_{2} \\
& <2 r_{k}
\end{aligned}
$$

onde

$$
d_{1}=\delta\left(\sum_{\mu \in \operatorname{supp} f_{i(k)}\left(b_{k}\right)} f_{i(k)}\left(b_{k}\right)(\mu) \cdot \psi_{k+1}(\mu)\right)
$$

$\mathrm{e}$

$$
d_{2}=\delta\left(\psi_{k}(i(k))\right)
$$

Como $r_{k} \rightarrow 0$, a seqüência $\left\{\phi_{J} 「_{\mathbb{Z}^{(E)}}\left(f_{\xi}\left(b_{k}\right)\right): k \in i^{-1}(\xi)\right\}$ converge para $\phi_{J} 「_{\mathbb{Z}^{(E)}}\left(\chi_{\xi}\right)$. Da propriedade (v) do lema 2.2.2 segue que

$$
\phi_{J} \uparrow_{\mathbb{Z}^{(E)}}\left(\chi_{\xi}\right)=p_{\xi}-\lim \left\{\phi_{J} \Upsilon_{\mathbb{Z}^{(E)}}\left(f_{\xi}(n)\right): n \in \omega\right\} .
$$

Logo, (ii) está verificada.

Resta mostrar que a seqüência $\left\{\phi_{J} \Upsilon_{\mathbb{Z}^{(E)}}\left(\chi_{n}\right): n \in \omega\right\}$ converge para $0+\mathbb{Z}$. Para tanto, basta observar que fixado $k \in \omega$, o conjunto $\left\{n \in \omega: \delta\left(\phi_{J} \uparrow_{\mathbb{Z}^{(E)}}\left(\chi_{n}\right), 0+\mathbb{Z}\right) \geq r_{k}\right\}$ é finito (uma vez que o mesmo está contido em $E_{k}$ ). Logo, (iii) está verificada.

Estamos prontos para estender $\phi_{J} \uparrow_{\mathbb{Z}^{(E)}}$ a um homomorfismo de grupos $\phi_{J}: \mathbb{Z}^{(\mathfrak{c})} \rightarrow \mathbb{T}$. Nossa única preocupação será garantir que $\phi_{J}\left(\chi_{\xi}\right)=p_{\xi}-\lim \left\{\phi_{J}\left(f_{\xi}(n)\right): n \in \omega\right\}$ para cada $\xi \in \mathfrak{c} \backslash E$, uma vez que supp $J \subset E$ e $\omega \subset E$.

Lema 2.2.4. Para cada $J \in \mathbb{Z}^{(\mathfrak{c})} \backslash\{0\}$, existe $\phi_{J}: \mathbb{Z}^{(\mathfrak{c})} \rightarrow \mathbb{T}$ um homomorfismo de grupos tal que:

(i) $\phi_{J}(J) \neq 0+\mathbb{Z}$;

(ii) $\phi_{J}\left(\chi_{\xi}\right)=p_{\xi}-\lim \left\{\phi_{J}\left(f_{\xi}(n)\right): n \in \omega\right\}$, para cada $\xi \in[\omega, \mathfrak{c}[$;

(iii) $\phi_{J}\left(\chi_{n}\right) \rightarrow 0+\mathbb{Z}$. 
Demonstração. Faremos a construção de $\phi_{J}$ por indução. De acordo com a proposição 2.2.1, existe $E \in[\mathfrak{c}]^{\omega}$ tal que $\operatorname{supp} J \subset E,|E \backslash \omega|=\omega$ e $\cup_{n \in \omega} \operatorname{supp} f_{\xi}(n) \subset E$, qualquer que seja $\xi \in E \backslash \omega$. Segundo o lema 2.2.3, é possível construir $\phi_{J} \uparrow_{\mathbb{Z}^{(E)}}: \mathbb{Z}^{(E)} \rightarrow \mathbb{T}$ um homomorfismo de grupos com as seguintes propriedades:

(1) $\phi_{J} \Upsilon_{\mathbb{Z}^{(E)}}(J) \neq 0+\mathbb{Z}$

(2) $\phi_{J} \Upsilon_{\mathbb{Z}^{(E)}}\left(\chi_{\xi}\right)=p_{\xi}-\lim \left\{\phi_{J} \Upsilon_{\mathbb{Z}^{(E)}}\left(f_{\xi}(n)\right): n \in \omega\right\}$, para cada $\xi \in E \backslash \omega ;$

(3) $\phi_{J} \uparrow_{\mathbb{Z}^{(E)}}\left(\chi_{n}\right) \rightarrow 0+\mathbb{Z}$.

Consideremos $\left\{\alpha_{\xi}: \xi<\mathfrak{c}\right\}$ uma enumeração estritamente crescente de $\mathfrak{c} \backslash E$. Definamos

$$
\tilde{\phi}_{J}\left(\chi_{\alpha_{0}}\right)=p_{\alpha_{0}}-\lim \left\{\phi_{J} \nwarrow_{\mathbb{Z}^{(E)}}\left(f_{\alpha_{0}}(n)\right): n \in \omega\right\}
$$

e façamos

$$
\tilde{\phi}_{J}(\tilde{J})=\phi_{J} \uparrow_{\mathbb{Z}^{(E)}}(\tilde{J})
$$

para todo $\tilde{J} \in \mathbb{Z}^{(E)} .^{9} \quad$ Note que a seqüência $\left\{\phi_{J} \Upsilon_{\mathbb{Z}^{(E)}}\left(f_{\alpha_{0}}(n)\right): n \in \omega\right\}$ admite $p_{\alpha_{0}}{ }^{-}$ limite em $\mathbb{T}$, uma vez que $\mathbb{T}$ é compacto. Estendamos $\tilde{\phi}_{J}$ a um homomorfismo de grupos $\phi_{J} \Upsilon_{\mathbb{Z}^{\left(E \cup\left\{\alpha_{0}\right\}\right)}}: \mathbb{Z}^{\left(E \cup\left\{\alpha_{0}\right\}\right)} \rightarrow \mathbb{T}$.

Repetindo indutivamente esta construção, obteremos $\phi_{J}: \mathbb{Z}^{(\mathfrak{c})} \rightarrow \mathbb{T}$ um homomorfismo de grupos satisfazendo (i), (ii) e (iii).

\subsection{Explicitando a topologia}

A próxima proposição mostra que o produto diagonal da família $\left\{\phi_{J}: J \in \mathbb{Z}^{(\mathfrak{c})} \backslash\{0\}\right\}$ é um monomorfismo de grupos.

Proposição 2.3.1. Suponhamos que, para cada $J \in \mathbb{Z}^{(\mathfrak{c})} \backslash\{0\}$, exista um homomorfismo de grupos $\phi_{J}: \mathbb{Z}^{(\mathfrak{c})} \rightarrow \mathbb{T}$ tal que $\phi_{J}(J) \neq 0+\mathbb{Z}$. A aplicação

$$
\begin{aligned}
\Phi: \mathbb{Z}^{(\mathfrak{c})} & \rightarrow \mathbb{T}^{\mathbb{Z}^{(\mathfrak{c})} \backslash\{0\}} \\
\tilde{J} & \mapsto \Phi(\tilde{J})
\end{aligned}
$$

dada por

$$
\Phi(\tilde{J})(J)=\phi_{J}(\tilde{J}), \text { para cada } J \in \mathbb{Z}^{(\mathfrak{c})} \backslash\{0\}
$$

\footnotetext{
${ }^{9}$ Estamos considerando $\mathbb{Z}^{(E)} \subset \mathbb{Z}^{\left(E \cup\left\{\alpha_{0}\right\}\right)} \subset \mathbb{Z}^{(\mathfrak{c})}$.
} 
é denominada produto diagonal da família $\left\{\phi_{J}: J \in \mathbb{Z}^{(\mathfrak{c})} \backslash\{0\}\right\}$ e é um monomorfismo de grupos.

Demonstração. Sejam $G, H \in \mathbb{Z}^{(\mathfrak{c})}$. Temos que $\Phi(G+H)(J)=\phi_{J}(G+H)=\phi_{J}(G)+\phi_{J}(H)=$ $\Phi(G)(J)+\Phi(H)(J)=[\Phi(G)+\Phi(H)](J)$, para todo $J \in \mathbb{Z}^{(\mathfrak{c})} \backslash\{0\}$. Logo, $\Phi$ é um homomorfismo de grupos. A fim de provar que $\Phi$ é injetora, basta mostrar que se $H \in \mathbb{Z}^{(\mathfrak{c})}$ é tal que $\Phi(H)=0$, então $H=0$. Suponhamos, portanto, que $H \in \mathbb{Z}^{(\mathfrak{c})}$ seja tal que $\Phi(H)=0$, ou seja, que $\Phi(H)(J)=0+\mathbb{Z}$ para todo $J \in \mathbb{Z}^{(\mathfrak{c})} \backslash\{0\}$. Então, $\phi_{J}(H)=0+\mathbb{Z}$, para todo $J \in \mathbb{Z}^{(\mathfrak{c})} \backslash\{0\}$. Como $\phi_{J}(J) \neq 0+\mathbb{Z}$ para cada $J \in \mathbb{Z}^{(\mathfrak{c})} \backslash\{0\}$, temos que $H \notin \mathbb{Z}^{(\mathfrak{c})} \backslash\{0\}$. Logo, $H=0$.

Teorema 2.3.2. O grupo abeliano livre de cardinalidade $\mathfrak{c}$ admite uma topologia enumeravelmente compacta com uma seqüência não trivial convergente que o torna um grupo topológico.

Demonstração. O grupo abeliano livre $\Phi\left[\mathbb{Z}^{(\mathfrak{c})}\right]$ munido da topologia de subespaço induzida por $\mathbb{T}^{\mathbb{Z}^{(\mathfrak{c})} \backslash\{0\}}$ é um grupo topológico. Não é difícil ver que $\Phi\left(\chi_{n}\right) \rightarrow 0$, uma vez que $\phi_{J}\left(\chi_{n}\right) \rightarrow 0+\mathbb{Z}$, para todo $J \in \mathbb{Z}^{(\mathfrak{c})} \backslash\{0\}$.

Consideremos $f: \omega \rightarrow \Phi\left[\mathbb{Z}^{(\mathfrak{c})}\right]$. Da proposição 2.1.2 segue que existe $j: \omega \rightarrow \omega$ estritamente crescente tal que $\Phi^{-1} \circ f \circ j$ é do tipo 1 ou $\Phi^{-1} \circ f \circ j=f_{1}+f_{2}$, onde $f_{2}$ é do tipo 2 ou constante e existe $m>0$ um número natural tal que, para cada $n \in \omega, f_{1}(n)$ é uma combinação linear com coeficientes pertencentes a $\{-1,0,1\}$ de $m$ elementos de $\left\{\chi_{n}: n \in \omega\right\}$. No primeiro caso temos que $\Phi^{-1} \circ f \circ j=f_{\xi}$ para algum $\xi \in\left[\omega, \mathfrak{c}\left[\mathrm{e}\right.\right.$, portanto, $\Phi\left(\chi_{\xi}\right)=p_{\xi}-\lim \left\{\Phi \circ f_{\xi}(n): n \in \omega\right\}$. Suponhamos agora que o segundo caso ocorra. Do fato de a seqüência $\left\{\Phi\left(\chi_{n}\right): n \in \omega\right\}$ ser convergente decorre que existe $\tilde{j}: \omega \rightarrow \omega$ estritamente crescente tal que $\Phi \circ f_{1} \circ \tilde{j}$ tem $p$-limite em $\Phi\left[\mathbb{Z}^{(\mathfrak{c})}\right]$, qualquer que seja $p \in \omega^{*}$. Uma vez que $f_{2}$ é do tipo 2 ou constante, temos que $f_{2} \circ \tilde{j}$ também o é e, portanto, $\Phi \circ f_{2} \circ \tilde{j}$ admite $p$-limite em $\Phi\left[\mathbb{Z}^{(\mathfrak{c})}\right]$ para algum $p \in \omega^{*}$. Como $f \circ j \circ \tilde{j}=\Phi \circ f_{1} \circ \tilde{j}+\Phi \circ f_{2} \circ \tilde{j}$, concluímos que $f \circ j \circ \tilde{j}$ tem $p$-limite em $\Phi\left[\mathbb{Z}^{(\mathfrak{c})}\right]$ e, portanto, $\Phi\left[\mathbb{Z}^{(\mathfrak{c})}\right]$ é enumeravelmente compacto. 
CAPÍTULO 3

\section{Uma topologia de grupo enumeravelmente compacta sem seqüências não triviais convergentes na reta real}

Neste capítulo, assumiremos a existência de $\mathfrak{c}$ ultrafiltros seletivos dois a dois incomparáveis (segundo a ordem de Rudin-Keisler) para mostrar que o grupo aditivo da reta real admite uma topologia enumeravelmente compacta sem seqüências não triviais convergentes que o torna um grupo topológico.

Como queremos aproveitar as idéias desenvolvidas no capítulo 2, começaremos identificando $\mathbb{R}$ com $\mathbb{Q}^{(\mathfrak{c})}$. Para cada $J \in \mathbb{Q}^{(\mathfrak{c})} \backslash\{0\}$, construiremos $\phi_{J}: \mathbb{Q}^{(\mathfrak{c})} \rightarrow \mathbb{T}$ um homomorfismo de grupos de modo que o produto diagonal da família $\left\{\phi_{J}: J \in \mathbb{Q}^{(\mathfrak{c})} \backslash\{0\}\right\}$ seja injetor e que a imagem de $\mathbb{Q}^{(\mathfrak{c})}$ por esta aplicação tenha as propriedades desejadas quando munida da topologia de subespaço induzida por $\mathbb{T}^{\mathbb{Q}^{(c)} \backslash\{0\}}$.

A principal diferença entre os capítulos 2 e 3 está, justamente, na construção dos homomorfismos $\phi_{J}$. Como os elementos de $\mathbb{T}$ têm mais de uma raiz $n$-ésima para cada $n>1$ número natural, precisaremos definir uma aplicação auxiliar adicional no lema 3.2.6 a fim de garantir que cada $\phi_{J}$ esteja bem-definido e seja um homomorfismo de grupos.

\subsection{Uma escolha adequada de seqüências}

Proposição 3.1.1. O grupo $(\mathbb{R},+)$ é isomorfo ao grupo $\left(\mathbb{Q}^{(\mathfrak{c})},+\right)$. 
Demonstração. Seja $B=\left\{x_{\xi}: \xi<\mathfrak{c}\right\}$ uma base ordenada de $\mathbb{R}$ como $\mathbb{Q}$-espaço vetorial. Para cada $x \in \mathbb{R} \backslash\{0\}$ existe um único $F_{x} \in[\mathfrak{c}]^{<\omega}$ não vazio e um único subconjunto $\left\{a_{\xi}: \xi \in F_{x}\right\}$ de $\mathbb{Q} \backslash\{0\}$ verificando

$$
x=\sum_{\xi \in F_{x}} a_{\xi} x_{\xi}
$$

Façamos $F_{0}=\emptyset$. A aplicação

$$
\begin{aligned}
\varphi: \mathbb{R} & \rightarrow \mathbb{Q}^{(\mathfrak{c})} \\
x & \mapsto f_{x}
\end{aligned}
$$

onde

$$
f_{x}(\xi)=\left\{\begin{array}{ccl}
a_{\xi} & \text { se } & \xi \in F_{x} \\
0 & \text { se } & \xi \in \mathfrak{c} \backslash F_{x}
\end{array}\right.
$$

é um isomorfismo de grupos. De fato, não há dúvidas de que $\varphi$ é bijetora. Sejam $x, y \in \mathbb{R}$. Se $x=0$ ou $y=0$, então $\varphi(x+y)=\varphi(x)+\varphi(y)$, uma vez que $\varphi(0)=f_{0}=0$. Suponhamos, portanto, $0 \neq x=\sum_{\xi \in F_{x}} a_{\xi} x_{\xi}$ e $0 \neq y=\sum_{\xi \in F_{y}} b_{\xi} x_{\xi}$. Temos que

$$
x+y=\sum_{\xi \in F_{x} \backslash F_{y}} a_{\xi} x_{\xi}+\sum_{\xi \in F_{y} \backslash F_{x}} b_{\xi} x_{\xi}+\sum_{\xi \in\left(F_{x} \cap F_{y}\right) \backslash I}\left(a_{\xi}+b_{\xi}\right) x_{\xi}
$$

onde

$$
I=\left\{\xi \in F_{x} \cap F_{y}: a_{\xi}+b_{\xi}=0\right\} .
$$

Logo, $f_{x+y}=f_{x}+f_{y}$, o que implica $\varphi(x+y)=\varphi(x)+\varphi(y)$.

Se $J \in \mathbb{Q}^{(\mathfrak{c})}$, então

$$
J=\sum_{\mu \in \operatorname{supp} J} J(\mu) \cdot \chi_{\mu}
$$

onde $\chi_{\mu}: \mathfrak{c} \rightarrow \mathbb{Q}$ é tal que

$$
\chi_{\mu}(\xi)=\left\{\begin{array}{lll}
1 & \text { se } & \xi=\mu \\
0 & \text { se } & \xi \neq \mu .
\end{array}\right.
$$

Dado $\mu \in \operatorname{supp} J$, podemos escrever

$$
J(\mu)=\frac{p(J, \mu)}{q(J, \mu)}
$$

onde $p(J, \mu), q(J, \mu) \in \mathbb{Z}, q(J, \mu)>0$ e $\operatorname{mdc}(p(J, \mu), q(J, \mu))=1$. Seja

$$
d(J)=\operatorname{mmc}\{q(J, \mu): \mu \in \operatorname{supp} J\}
$$


e, para cada $\mu \in \operatorname{supp} J$, seja

$$
a(J, \mu)=p(J, \mu) \cdot \frac{d(J)}{q(J, \mu)} .
$$

Por fim, sejam

$$
\begin{aligned}
|q(J)| & =\max \{q(J, \mu): \mu \in \operatorname{supp} J\} \\
|p(J)| & =\max \{|p(J, \mu)|: \mu \in \operatorname{supp} J\} \\
|a(J)| & =\max \{|a(J, \mu)|: \mu \in \operatorname{supp} J\}
\end{aligned}
$$

e

$$
\|a(J)\|=\sum_{\mu \in \operatorname{supp} J}|a(J, \mu)|
$$

Denotaremos por $\mathcal{F}$ o conjunto de todas as funções $f: \omega \rightarrow \mathbb{Q}^{(\mathfrak{c})}$ que satisfazem uma das seguintes condições:

(1) $\operatorname{supp} f(n) \backslash \cup_{m<n} \operatorname{supp} f(m) \neq \emptyset$, para todo $n \in \omega$;

(2) $|q(f(n))|>n$, para todo $n \in \omega$;

(3) $\{|q(f(n))|: n \in \omega\}$ é limitado e $|p(f(n))|>n$, para todo $n \in \omega$.

Definição 3.1.2. Sejam $f \in \mathcal{F}$ e $i \in\{1,2,3\}$. Dizemos que $f$ é do tipo $i$ se $f$ satisfaz a condição (i) descrita acima.

A próxima proposição mostra que é possível recuperar uma subseqüência de cada seqüência não trivial em $\mathbb{Q}^{(\mathfrak{c})}$ através de $\mathcal{F}$.

Proposição 3.1.3. Se $f: \omega \rightarrow \mathbb{Q}^{(\mathfrak{c})}$, então existe $j: \omega \rightarrow \omega$ estritamente crescente tal que $f \circ j$ é constante ou é de um dos tipos acima mencionados.

Demonstração. Há quatro casos a serem considerados.

Caso 1: $\cup_{n \in \omega} \operatorname{supp} f(n)$ é infinito.

Seja $n_{0}=0$. Fixemos $k \in \omega$ e suponhamos definidos $n_{0}, n_{1}, \ldots, n_{k}$. Uma vez que $\cup_{n \in \omega} \operatorname{supp} f(n)$ é infinito, existe um número natural $n_{k+1}>n_{k}$ tal que supp $f\left(n_{k+1}\right) \backslash$ $\cup_{l<k+1} \operatorname{supp} f\left(n_{l}\right) \neq \emptyset$. Temos que

$$
\begin{aligned}
j: \omega & \rightarrow \omega \\
k & \mapsto n_{k}
\end{aligned}
$$


é uma função estritamente crescente tal que $f \circ j$ é do tipo 1 .

Caso 2: $\cup_{n \in \omega} \operatorname{supp} f(n)$ é finito e $\{|q(f(n))|: n \in \omega\}$ é ilimitado.

Por indução, tomemos $\left\{n_{k}: k \in \omega\right\}$ uma seqüência estritamente crescente de números naturais tal que $\left|q\left(f\left(n_{k}\right)\right)\right|>k$, para todo $k \in \omega$. Temos que

$$
\begin{aligned}
j: \omega & \rightarrow \omega \\
k & \mapsto n_{k}
\end{aligned}
$$

é uma função estritamente crescente tal que $f \circ j$ é do tipo 2 .

Caso 3: $\cup_{n \in \omega} \operatorname{supp} f(n)$ é finito, $\{|q(f(n))|: n \in \omega\}$ é limitado e $\{|p(f(n))|: n \in \omega\}$ é ilimitado. Por indução, tomemos $\left\{n_{k}: k \in \omega\right\}$ uma seqüência estritamente crescente de números naturais tal que $\left|p\left(f\left(n_{k}\right)\right)\right|>k$, para todo $k \in \omega$. Temos que

$$
\begin{aligned}
j: \omega & \rightarrow \omega \\
k & \mapsto n_{k}
\end{aligned}
$$

é uma função estritamente crescente tal que $f \circ j$ é do tipo 3 .

Caso 4: $\cup_{n \in \omega} \operatorname{supp} f(n)$ é finito, $\{|p(f(n))|: n \in \omega\}$ e $\{|q(f(n))|: n \in \omega\}$ são limitados. Temos que $\{f(n): n \in \omega\}$ é um conjunto finito e, portanto, $f$ possui uma subseqüência constante.

Proposição 3.1.4. Existe $\left\{f_{\xi}: 0<\xi<\mathfrak{c}\right\}$ uma indexação de $\mathcal{F}$ tal que $\cup_{n \in \omega} \operatorname{supp} f_{\xi}(n) \subset \xi$, para todo $\xi \in] 0, \mathfrak{c}[$.

Demonstração. Análoga à da proposição 2.1.3.

\subsection{Construindo homomorfismos de grupos}

Sejam $\left\{p_{\xi}: 0<\xi<\mathfrak{c}\right\}$ uma família de ultrafiltros seletivos dois a dois incomparáveis segundo a ordem de Rudin-Keisler e $\left\{f_{\xi}: 0<\xi<\mathfrak{c}\right\}$ uma indexação de $\mathcal{F}$ tal que $\cup_{n \in \omega} \operatorname{supp} f_{\xi}(n) \subset \xi$, qualquer que seja $\xi \in] 0, \mathfrak{c}[$.

Proposição 3.2.1. Seja $J \in \mathbb{Q}^{(\mathfrak{c})} \backslash\{0\}$. Existe $E \in[\mathfrak{c}]^{\omega}$ tal que supp $J \subset E$ e $\cup_{n \in \omega} \operatorname{supp} f_{\xi}(n) \subset$ E, para cada $\xi \in E \backslash\{0\}$. 
Demonstração. Análoga à da proposição 2.2.1.

Lema 3.2.2. Sejam $J \in \mathbb{Q}^{(\mathfrak{c})} \backslash\{0\}$ e $E \in[\mathfrak{c}]^{\omega}$ tais que $\operatorname{supp} J \subset E$ e $\cup_{n \in \omega} \operatorname{supp} f_{\xi}(n) \subset E$, para cada $\xi \in E \backslash\{0\}$. Existe $\left\{E_{k}: k \in \omega\right\}$ uma familia de subconjuntos finitos de $E,\left\{b_{k}: k \in \omega\right\}$ uma seqüência estritamente crescente de números naturais, $\left\{r_{k}: k \in \omega\right\}$ uma seqüência de números reais positivos e $i: \omega \rightarrow E \backslash\{0\}$ tais que:

(i) $\operatorname{supp} J \subset E_{0}$;

(ii) $E=\cup_{k \in \omega} E_{k}$;

(iii) $i(k) \in E_{k}$, para todo $k \in \omega$;

(iv) $E_{k+1} \supset E_{k} \cup \bigcup\left\{\operatorname{supp} f_{i(m)}\left(b_{m}\right): m \leq k\right\}$, para todo $k \in \omega$;

(v) $\left\{b_{k}: k \in i^{-1}(\{\xi\})\right\} \in p_{\xi}$, para todo $\xi \in E \backslash\{0\}$;

(vi) Se $f_{i(k)}$ é do tipo 1, então $\operatorname{supp} f_{i(k)}\left(b_{k}\right) \backslash E_{k} \neq \emptyset$;

(vii) Se $f_{i(k)}$ é do tipo 2, então $\left|q\left(f_{i(k)}\left(b_{k}\right)\right)\right| \cdot r_{k}>d(J) \cdot \prod_{m<k} d\left(f_{i(m)}\left(b_{m}\right)\right)$;

(viii) Se $f_{i(k)}$ é do tipo 3, então $\left|a\left(f_{i(k)}\left(b_{k}\right)\right)\right| \cdot r_{k}>4 \cdot d\left(f_{i(k)}\left(b_{k}\right)\right)$;

(ix) $r_{0}=\frac{1}{4 \cdot\|a(J)\|}$;

(x) $r_{k+1}=\frac{r_{k}}{2 \cdot\left\|a\left(f_{i(k)}\left(b_{k}\right)\right)\right\|}$, para todo $k \in \omega$.

Demonstração. Consideremos $\left\{a_{n}: n \in \omega\right\}$ uma enumeração de $E$. Sejam $F_{0}=\operatorname{supp} J \cup\left\{a_{0}\right\}$ e $F_{n+1}=F_{n} \cup \bigcup\left\{\operatorname{supp} f_{\xi}(m): m \leq n, \xi \in F_{n} \backslash\{0\}\right\} \cup\left\{a_{n+1}\right\}$, para cada $n \in \omega$. Temos que $\left\{F_{n}: n \in \omega\right\}$ é uma família de subconjuntos finitos de $E$ tal que:

(1) $\operatorname{supp} J \subset F_{0}$;

(2) $E=\cup_{n \in \omega} F_{n}$;

(3) $F_{n+1} \supset F_{n} \cup \bigcup\left\{\operatorname{supp} f_{\xi}(m): m \leq n, \xi \in F_{n} \backslash\{0\}\right\}$.

Fixemos $\xi \in E \backslash\{0\}$ e $n \in \omega$. Se $f_{\xi}$ é do tipo 1, definimos

$$
A_{n}^{\xi}=\left\{k \in \omega: \operatorname{supp} f_{\xi}(k) \backslash F_{n} \neq \emptyset\right\}
$$


Se $f_{\xi}$ é do tipo 2 , definimos

$$
A_{n}^{\xi}=\left\{k \in \omega:\left|q\left(f_{\xi}(k)\right)\right|>2^{n+2} \cdot X_{n-1}\right\}
$$

onde

$$
X_{m}=\|a(J)\| \cdot d(J) \cdot \prod_{\substack{l \leq m \\ \zeta \in F_{m} \backslash\{0\}}} d\left(f_{\zeta}(l)\right) \cdot \prod_{\substack{l \leq m \\ \zeta \in F_{m} \backslash\{0\}}}\left\|a\left(f_{\zeta}(l)\right)\right\|
$$

para todo $m \in \omega \mathrm{e}$

$$
X_{-1}=\|a(J)\| \cdot d(J) .
$$

Se $f_{\xi}$ é do tipo 3, então $\left|a\left(f_{\xi}(n)\right)\right|>n$ para cada $n \in \omega$ e $\left\{\left|q\left(f_{\xi}(n)\right)\right|: n \in \omega\right\}$ é limitado. Tomemos $M_{\xi} \in \omega$ tal que $\left|q\left(f_{\xi}(n)\right)\right| \leq M_{\xi}$, para todo $n \in \omega$. Definimos

$$
A_{n}^{\xi}=\left\{k \in \omega:\left|a\left(f_{\xi}(k)\right)\right|>2^{n+4} \cdot M_{\xi} ! \cdot Y_{n-1}\right\}
$$

onde

$$
Y_{m}=\|a(J)\| \cdot \prod_{\substack{l \leq m \\ \zeta \in F_{m} \backslash\{0\}}}\left\|a\left(f_{\zeta}(l)\right)\right\|
$$

para todo $m \in \omega$ e

$$
Y_{-1}=\|a(J)\|
$$

Observamos que $A_{n}^{\xi}$ é um subconjunto cofinito de $\omega$, quaisquer que sejam $n \in \omega$ e $\xi \in E \backslash\{0\}$. Logo, para cada $\xi \in E \backslash\{0\}$, temos que $\left\{A_{n}^{\xi}: n \in \omega\right\} \subset p_{\xi}$, uma vez que $p_{\xi}$ é um ultrafiltro livre sobre $\omega$. Da seletividade de $p_{\xi}$ decorre que existe uma seqüência $\left\{a_{n}^{\xi}: n \in \omega\right\} \in p_{\xi}$ tal que $a_{n}^{\xi} \in A_{n}^{\xi}$ e $n<a_{n}^{\xi}$, para cada $n \in \omega$.

Do lema 1.5.4 segue que existe $\left\{I_{\xi}: \xi \in E \backslash\{0\}\right\}$ uma família de subconjuntos de $\omega$ dois a dois disjuntos tal que:

(a) $\left\{a_{n}^{\xi}: n \in I_{\xi}\right\} \in p_{\xi}$, para cada $\xi \in E \backslash\{0\}$;

(b) $\left\{\left[n, a_{n}^{\xi}\right]: n \in I_{\xi}, \xi \in E \backslash\{0\}\right\}$ é uma família de intervalos de $\omega$ dois a dois disjuntos.

Para cada $\xi \in E \backslash\{0\}$, seja

(c) $N_{\xi}=\min \left\{n \in \omega: \xi \in F_{n}\right\}$.

Podemos supor, sem perda de generalidade, que 
(d) $N_{\xi}<n$, para todo $n \in I_{\xi}$.

Consideremos $\left\{n_{k}: k \in \omega\right\}$ uma enumeração estritamente crescente de $\dot{\cup}_{\xi \in E \backslash\{0\}} I_{\xi}$. Seja

$$
\begin{aligned}
i: \omega & \rightarrow E \backslash\{0\} \\
k & \mapsto i(k)
\end{aligned}
$$

onde $i(k)$ é o único elemento de $E \backslash\{0\}$ tal que $n_{k} \in I_{i(k)}$.

Para cada $k \in \omega$, façamos $b_{k}=a_{n_{k}}^{i(k)}, E_{k}=F_{n_{k}}$ e definamos $r_{k}$ segundo os ítens (ix) e (x) do enunciado deste lema. Resta mostrar que as condições (i)-(viii) estão satisfeitas.

(i) Como $0 \leq n_{0}$, temos que $E_{0}=F_{n_{0}} \supset F_{0} \supset \operatorname{supp} J$.

(ii) $\cup_{k \in \omega} E_{k}=\cup_{k \in \omega} F_{n_{k}}=E$.

(iii) Seja $k \in \omega$. Temos que $n_{k} \in I_{i(k)}$. De (d) segue que $N_{i(k)}<n_{k}$. De (c) e (3) vem que $i(k) \in F_{N_{i(k)}} \subset F_{n_{k}}=E_{k}$.

(iv) De (3) vem que $E_{k} \subset E_{k+1}$, pois $\left\{n_{k}: k \in \omega\right\}$ é uma enumeração estritamente crescente de $\cup_{\xi \in E \backslash\{0\}} I_{\xi}$. Resta mostrar que supp $f_{i(m)}\left(b_{m}\right) \subset E_{k+1}$, para cada $m \leq k$. De (3) decorre que

$$
E_{k+1}=F_{n_{k+1}} \supset \bigcup\left\{\operatorname{supp} f_{\xi}(m): m \leq n_{k+1}-1, \xi \in F_{n_{k+1}-1} \backslash\{0\}\right\} .
$$

Por (iii), $i(m) \in E_{m} \subset E_{k}=F_{n_{k}} \subset F_{n_{k+1}-1}$, para todo $m \leq k$. Além disso, $i(m) \neq 0$. Por (b), $b_{m}=a_{n_{m}}^{i(m)} \leq n_{k+1}-1$, para todo $m \leq k$. Logo, $\operatorname{supp} f_{i(m)}\left(b_{m}\right) \subset E_{k+1}$.

(v) Seja $\xi \in E \backslash\{0\}$. Temos que $\left\{b_{k}: k \in i^{-1}(\{\xi\})\right\}=\left\{a_{n_{k}}^{i(k)}: k \in i^{-1}(\{\xi\})\right\}=\left\{a_{n}^{\xi}: n \in I_{\xi}\right\} \in$ $p_{\xi}$, por (a).

(vi) Suponhamos que $f_{i(k)}$ seja do tipo 1. Temos que $b_{k}=a_{n_{k}}^{i(k)} \in A_{n_{k}}^{i(k)}$. Da definição de $A_{n_{k}}^{i(k)}$ vem que

$$
\operatorname{supp} f_{i(k)}\left(b_{k}\right) \backslash E_{k}=\operatorname{supp} f_{i(k)}\left(b_{k}\right) \backslash F_{n_{k}} \neq \emptyset \text {. }
$$

(vii) Suponhamos que $f_{i(k)}$ seja do tipo 2. Temos que $b_{k}=a_{n_{k}}^{i(k)} \in A_{n_{k}}^{i(k)}$. Da definição de $A_{n_{k}}^{i(k)}$ vem que

$$
\left|q\left(f_{i(k)}\left(b_{k}\right)\right)\right|>2^{n_{k}+2} \cdot X_{n_{k}-1}
$$


onde

$$
X_{n_{k}-1}=\|a(J)\| \cdot d(J) \cdot \prod_{\substack{m \leq n_{k}-1 \\ \xi \in F_{n_{k}-1} \backslash\{0\}}} d\left(f_{\xi}(m)\right) \cdot \prod_{\substack{m \leq n_{k}-1 \\ \xi \in F_{n_{k}-1} \backslash\{0\}}}\left\|a\left(f_{\xi}(m)\right)\right\| .
$$

Observamos que se $m<k$, então $n_{m} \leq n_{k-1}$ e, portanto, $i(m) \in E_{m}=F_{n_{m}} \subset F_{n_{k-1}} \subset$ $F_{n_{k}-1}$. Note que $i(m) \neq 0$. Como $b_{m}=a_{n_{m}}^{i(m)}$ e $\left[n_{m}, a_{n_{m}}^{i(m)}\right] \cap\left[n_{k}, a_{n_{k}}^{i(k)}\right]=\emptyset$, temos que $b_{m}<n_{k}$ e, portanto, $b_{m} \leq n_{k}-1$. Uma vez que

$$
r_{k}=\frac{1}{2^{k+2} \cdot\|a(J)\| \cdot \prod_{m<k}\left\|a\left(f_{i(m)}\left(b_{m}\right)\right)\right\|}
$$

concluímos que

$$
\left|q\left(f_{i(k)}\left(b_{k}\right)\right)\right| \cdot r_{k}>d(J) \cdot \prod_{m<k} d\left(f_{i(m)}\left(b_{m}\right)\right) .
$$

(viii) Suponhamos que $f_{i(k)}$ seja do tipo 3. Um argumento análogo ao apresentado em (vii) nos permite concluir que

$$
\left|a\left(f_{i(k)}\left(b_{k}\right)\right)\right| \cdot r_{k}>4 \cdot M_{i(k)} ! \geq 4 \cdot d\left(f_{i(k)}\left(b_{k}\right)\right) .
$$

Os próximos três lemas compreendem a parte técnica referente aos tipos 1, 2 e 3 .

Lema 3.2.3. Sejam $I \in[\mathfrak{c}]^{\mathfrak{c}}, c \in \mathbb{N} \backslash\{0\}, \epsilon>0, A \in \mathcal{B}, F \in[I]^{<\omega}, J \in \mathbb{Q}^{(I)}, \mu \in \operatorname{supp} J \backslash F e$ $\psi: F \rightarrow \mathcal{B} \backslash\{\mathbb{T}\} \operatorname{com} \delta(\psi(\xi)) \geq \epsilon / c$, para todo $\xi \in F$. Seja $\tilde{F}=F \cup \operatorname{supp} J$. Existe $\tilde{\psi}: \tilde{F} \rightarrow \mathcal{B}$ satisfazendo as seguintes condições:

(i) $d(J) \cdot \bar{\psi}(\xi) \subset \psi(\xi)$, para todo $\xi \in F$;

(ii) $\delta(\tilde{\psi}(\xi))=\frac{\epsilon}{2 \cdot\|a(J)\| \cdot c \cdot d(J)}$, para todo $\xi \in \tilde{F}$;

(iii) $\delta\left(\sum_{\xi \in \operatorname{supp} J} a(J, \xi) \cdot c \cdot \tilde{\psi}(\xi)\right)<\epsilon$;

(iv) $A \cap \sum_{\xi \in \operatorname{supp} J} a(J, \xi) \cdot c \cdot \tilde{\psi}(\xi) \neq \emptyset$.

Demonstração. Se $\xi \in \tilde{F} \backslash \operatorname{supp} J \subset F$, fixemos uma raiz $d(J)$-ésima do ponto médio de $\psi(\xi)$ e definamos $\tilde{\psi}(\xi)$ como sendo o arco aberto de $\mathbb{T}$ centrado na raiz em questão com diâmetro

$$
\delta(\tilde{\psi}(\xi))=\frac{\epsilon}{2 \cdot\|a(J)\| \cdot c \cdot d(J)} .
$$


Se $\xi \in \operatorname{supp} J \backslash\{\mu\}$, fixemos $z_{\xi} \in \mathbb{T}$ da seguinte maneira: se $\xi \in F$, então $z_{\xi}$ é uma raiz $d(J)$-ésima do ponto médio de $\psi(\xi)$; se $\xi \notin F$, então $z_{\xi}$ é um elemento arbitrário de $\mathbb{T}$. Seja

$$
x=\sum_{\xi \in \operatorname{supp} J \backslash\{\mu\}} a(J, \xi) \cdot c \cdot z_{\xi} .
$$

Tomemos $z_{\mu} \in \mathbb{T}$ tal que

$$
x+a(J, \mu) \cdot c \cdot z_{\mu} \in A .
$$

Para cada $\xi \in \operatorname{supp} J$, definimos $\tilde{\psi}(\xi)$ como sendo o arco aberto de $\mathbb{T}$ centrado em $z_{\xi}$ com diâmetro

$$
\delta(\tilde{\psi}(\xi))=\frac{\epsilon}{2 \cdot\|a(J)\| \cdot c \cdot d(J)} .
$$

As condições (i), (ii), (iii) e (iv) estão verificadas. De fato, é evidente que (i) e (ii) estão satisfeitas, uma vez que para cada $\xi \in F, z_{\xi}$ é uma raiz $d(J)$-ésima do ponto médio de $\psi(\xi)$. A condição (iii) é uma conseqüência de (ii). Por fim, a validade de (iv) decorre da escolha de $z_{\mu}$.

Lema 3.2.4. Sejam $I \in[\mathfrak{c}]^{\mathfrak{c}}, c \in \mathbb{N} \backslash\{0\}, \epsilon>0, A \in \mathcal{B} \operatorname{com} \delta(A) \geq \epsilon, F \in[I]^{<\omega}, J \in \mathbb{Q}^{(I)}$, $\mu \in F \cap \operatorname{supp} J \operatorname{com} q(J, \mu) \cdot \epsilon>c$ e $\psi: F \rightarrow \mathcal{B} \backslash\{\mathbb{T}\} \operatorname{com} \delta(\psi(\xi)) \geq \epsilon / c$, para todo $\xi \in F$. Seja $\tilde{F}=F \cup \operatorname{supp} J$. Existe $\tilde{\psi}: \tilde{F} \rightarrow \mathcal{B}$ satisfazendo as seguintes condições:

(i) $d(J) \cdot \bar{\psi}(\xi) \subset \psi(\xi)$, para todo $\xi \in F$;

(ii) $\delta(\tilde{\psi}(\xi))=\frac{\epsilon}{2 \cdot\|a(J)\| \cdot c \cdot d(J)}$, para todo $\xi \in \tilde{F}$;

(iii) $\delta\left(\sum_{\xi \in \operatorname{supp} J} a(J, \xi) \cdot c \cdot \tilde{\psi}(\xi)\right)<\epsilon$;

(iv) $A \cap \sum_{\xi \in \operatorname{supp} J} a(J, \xi) \cdot c \cdot \tilde{\psi}(\xi) \neq \emptyset$.

Demonstração. Se $\xi \in \tilde{F} \backslash \operatorname{supp} J \subset F$, fixemos uma raiz $d(J)$-ésima do ponto médio de $\psi(\xi)$ e definamos $\tilde{\psi}(\xi)$ como sendo o arco aberto de $\mathbb{T}$ centrado na raiz em questão com diâmetro

$$
\delta(\tilde{\psi}(\xi))=\frac{\epsilon}{2 \cdot\|a(J)\| \cdot c \cdot d(J)}
$$

Se $\xi \in \operatorname{supp} J \backslash\{\mu\}$, fixemos $z_{\xi} \in \mathbb{T}$ da seguinte maneira: se $\xi \in F$, então $z_{\xi}$ é uma raiz $d(J)$-ésima do ponto médio de $\psi(\xi)$; se $\xi \notin F$, então $z_{\xi}$ é um elemento arbitrário de $\mathbb{T}$. Seja

$$
x=\sum_{\xi \in \operatorname{supp} J \backslash\{\mu\}} a(J, \xi) \cdot c \cdot z_{\xi} .
$$


Denotemos por $x_{\mu}$ o ponto médio de $\psi(\mu)$. Existem $d(J)$ raízes $d(J)$-ésimas de $x_{\mu}$. Se multiplicarmos cada uma delas por $a(J, \mu) \cdot c$ teremos

$$
\frac{q(J, \mu)}{\operatorname{mdc}(c, q(J, \mu))}
$$

novos elementos, uma vez que $p(J, \mu)$ e $q(J, \mu)$ são primos entre si. De

$$
\frac{q(J, \mu)}{\operatorname{mdc}(c, q(J, \mu))} \geq \frac{q(J, \mu)}{c}>\frac{1}{\epsilon}
$$

segue que a distância entre dois desses novos elementos consecutivos é menor que $\epsilon$. Como $\delta(A-x)=\delta(A) \geq \epsilon$, existe $z_{\mu} \in \mathbb{T}$ tal que $d(J) \cdot z_{\mu}=x_{\mu}$ e

$$
x+a(J, \mu) \cdot c \cdot z_{\mu} \in A .
$$

Para cada $\xi \in \operatorname{supp} J$, definimos $\tilde{\psi}(\xi)$ como sendo o arco aberto de $\mathbb{T}$ centrado em $z_{\xi}$ com diâmetro

$$
\delta(\tilde{\psi}(\xi))=\frac{\epsilon}{2 \cdot\|a(J)\| \cdot c \cdot d(J)} .
$$

As condições (i), (ii), (iii) e (iv) estão verificadas. De fato, é evidente que (i) e (ii) estão satisfeitas, uma vez que para cada $\xi \in F, z_{\xi}$ é uma raiz $d(J)$-ésima do ponto médio de $\psi(\xi)$. A condição (iii) é uma conseqüência de (ii). Por fim, a validade de (iv) decorre da escolha de $z_{\mu}$.

Lema 3.2.5. Sejam $I \in[\mathfrak{c}]^{\mathfrak{c}}, c \in \mathbb{N} \backslash\{0\}, \epsilon>0, A \in \mathcal{B}, F \in[I]^{<\omega}, J \in \mathbb{Q}^{(I)}, \mu \in F \cap \operatorname{supp} J$ com $|a(J, \mu)| \cdot \epsilon>4 \cdot d(J)$ e $\psi: F \rightarrow \mathcal{B} \backslash\{\mathbb{T}\} \operatorname{com} \delta(\psi(\xi)) \geq \epsilon / c$, para todo $\xi \in F$. Seja $\tilde{F}=F \cup \operatorname{supp} J$. Existe $\tilde{\psi}: \tilde{F} \rightarrow \mathcal{B}$ satisfazendo as seguintes condições:

(i) $d(J) \cdot \bar{\psi}(\xi) \subset \psi(\xi)$, para todo $\xi \in F$;

(ii) $\delta(\tilde{\psi}(\xi))=\frac{\epsilon}{2 \cdot\|a(J)\| \cdot c \cdot d(J)}$, para todo $\xi \in \tilde{F}$;

(iii) $\delta\left(\sum_{\xi \in \operatorname{supp} J} a(J, \xi) \cdot c \cdot \tilde{\psi}(\xi)\right)<\epsilon$;

(iv) $A \cap \sum_{\xi \in \operatorname{supp} J} a(J, \xi) \cdot c \cdot \tilde{\psi}(\xi) \neq \emptyset$.

Demonstração. Se $\xi \in \tilde{F} \backslash \operatorname{supp} J \subset F$, fixemos uma raiz $d(J)$-ésima do ponto médio de $\psi(\xi)$ e 
definamos $\tilde{\psi}(\xi)$ como sendo o arco aberto de $\mathbb{T}$ centrado na raiz em questão com diâmetro

$$
\delta(\tilde{\psi}(\xi))=\frac{\epsilon}{2 \cdot\|a(J)\| \cdot c \cdot d(J)}
$$

Se $\xi \in \operatorname{supp} J \backslash\{\mu\}$, fixemos $z_{\xi} \in \mathbb{T}$ da seguinte maneira: se $\xi \in F$, então $z_{\xi}$ é uma raiz $d(J)$-ésima do ponto médio de $\psi(\xi)$; se $\xi \notin F$, então $z_{\xi}$ é um elemento arbitrário de $\mathbb{T}$. Seja

$$
x=\sum_{\xi \in \operatorname{supp} J \backslash\{\mu\}} a(J, \xi) \cdot c \cdot z_{\xi} .
$$

Denotemos por $x_{\mu}$ o ponto médio de $\psi(\mu)$. Fixemos uma raiz $d(J)$-ésima de $x_{\mu}$ e, centrado nela, consideremos $\tilde{A}$ o arco aberto de $\mathbb{T}$ com diâmetro

$$
\delta(\tilde{A})=\frac{\epsilon}{4 \cdot c \cdot d(J)}
$$

Se multiplicarmos $\tilde{A}$ por $a(J, \mu) \cdot c$ obteremos $\mathbb{T}$, uma vez que $|a(J, \mu)| \cdot \epsilon>4 \cdot d(J)$. Portanto, existe $z_{\mu} \in \tilde{A}$ tal que

$$
x+a(J, \mu) \cdot c \cdot z_{\mu} \in A .
$$

Para cada $\xi \in \operatorname{supp} J$, definimos $\tilde{\psi}(\xi)$ como sendo o arco aberto de $\mathbb{T}$ centrado em $z_{\xi}$ com diâmetro

$$
\delta(\tilde{\psi}(\xi))=\frac{\epsilon}{2 \cdot\|a(J)\| \cdot c \cdot d(J)} .
$$

As condições (i), (ii), (iii) e (iv) estão verificadas. De fato, temos que (i) e (ii) estão satisfeitas, já que $\tilde{A}$ é suficientemente pequeno para que $d(J) \cdot \tilde{A}$ esteja contido em $\psi(\xi)$ e $a(J, \mu) \cdot c$ é suficientemente grande para que $a(J, \mu) \cdot c \cdot \tilde{A}$ seja igual a $\mathbb{T}$. A condição (iii) é uma conseqüência de (ii). Por fim, a validade de (iv) decorre da escolha de $z_{\mu}$.

Lema 3.2.6. Sejam $J \in \mathbb{Q}^{(\mathfrak{c})} \backslash\{0\}$ e $E \in[\mathfrak{c}]^{\omega}$ tais que $\operatorname{supp} J \subset E$ e $\cup_{n \in \omega} \operatorname{supp} f_{\xi}(n) \subset E$, para cada $\xi \in E \backslash\{0\}$. Existe $\phi_{J} \Gamma_{\mathbb{Q}^{(E)}}: \mathbb{Q}^{(E)} \rightarrow \mathbb{T}$ um homomorfismo de grupos com as seguintes propriedades:

(i) $\phi_{J} \uparrow_{\mathbb{Q}^{(E)}}(J) \neq 0+\mathbb{Z}$;

(ii) $\phi_{J} \Upsilon_{\mathbb{Q}^{(E)}}\left(\chi_{\xi}\right)=p_{\xi}-\lim \left\{\phi_{J} \Upsilon_{\mathbb{Q}^{(E)}}\left(f_{\xi}(n)\right): n \in \omega\right\}$, para cada $\xi \in E \backslash\{0\} .{ }^{1}$

\footnotetext{
${ }^{1}$ Estamos considerando $\mathbb{Q}^{(E)} \subset \mathbb{Q}^{(\mathfrak{c})}$.
} 
Demonstração. Consideremos $\left\{E_{n}: n \in \omega\right\},\left\{b_{n}: n \in \omega\right\},\left\{r_{n}: n \in \omega\right\}$ e $i: \omega \rightarrow E \backslash\{0\}$ de acordo com o lema 3.2.2. A fim de exibirmos um homomorfismo de grupos $\phi_{J} \uparrow_{\mathbb{Q}^{(E)}}$ satisfazendo as condições (i) e (ii) deste lema, construiremos indutivamente aplicações auxiliares $\psi_{n}: E \rightarrow \mathcal{B}$ e $\psi_{n}^{*}: E_{n} \rightarrow \mathcal{B}$, para cada $n \in \omega$.

Para cada $\xi \in E_{0}$, tomemos $y_{\xi} \in \mathbb{R}$ tal que

$$
\sum_{\xi \in \operatorname{supp} J} J(\xi) \cdot y_{\xi}=\frac{1}{2}
$$

e façamos

$$
x_{\xi}=\frac{1}{d(J)} \cdot y_{\xi}+\mathbb{Z}
$$

Temos que

$$
\sum_{\xi \in \operatorname{supp}} a(J, \xi) \cdot x_{\xi}=\frac{1}{2}+\mathbb{Z}
$$

Seja $\psi_{0}^{*}(\xi)$ o arco aberto de $\mathbb{T}$ centrado em $x_{\xi}$ com diâmetro

$$
\delta\left(\psi_{0}^{*}(\xi)\right)=\frac{r_{0}}{d(J)}
$$

Seja, também, $\psi_{0}(\xi)=d(J) \cdot \psi_{0}^{*}(\xi)$. Claramente, $\delta\left(\psi_{0}(\xi)\right)=r_{0}$. Uma vez que

$$
\frac{1}{2}+\mathbb{Z} \in \sum_{\xi \in \operatorname{supp} J} a(J, \xi) \cdot \psi_{0}^{*}(\xi)
$$

e

$$
\delta\left(\sum_{\xi \in \operatorname{supp} J} a(J, \xi) \cdot \psi_{0}^{*}(\xi)\right) \leq \sum_{\xi \in \operatorname{supp} J}|a(J, \xi)| \cdot \delta\left(\psi_{0}^{*}(\xi)\right) \leq \frac{1}{4}
$$

concluímos que

$$
0+\mathbb{Z} \notin \sum_{\xi \in \operatorname{supp} J} a(J, \xi) \cdot \psi_{0}^{*}(\xi)
$$

Por fim, se $\xi \in E \backslash E_{0}$, façamos $\psi_{0}(\xi)=\mathbb{T}$.

Seja $n \in \omega$. Suponhamos definidas $\psi_{n}: E \rightarrow \mathcal{B}$ e $\psi_{n}^{*}: E_{n} \rightarrow \mathcal{B}$. Vamos construir $\psi_{n+1}: E \rightarrow \mathcal{B}$ e $\psi_{n+1}^{*}: E_{n+1} \rightarrow \mathcal{B}$ com as seguintes propriedades:

(1) Se $\xi \in E \backslash E_{n+1}$, então $\psi_{n+1}(\xi)=\mathbb{T}$;

(2) Se $\xi \in E_{n+1} \backslash E_{n}$, então $\psi_{n+1}^{*}(\xi)$ é tal que: 
(a) $d(J) \cdot \prod_{m<n+1} d\left(f_{i(m)}\left(b_{m}\right)\right) \cdot \overline{\psi_{n+1}^{*}(\xi)} \subset \psi_{n}(\xi)$;

(b) $\delta\left(\psi_{n+1}^{*}(\xi)\right)=\frac{r_{n+1}}{d(J) \cdot \prod_{m<n+1} d\left(f_{i(m)}\left(b_{m}\right)\right)}$.

Neste caso, façamos

$$
\psi_{n+1}(\xi)=d(J) \cdot \prod_{m<n+1} d\left(f_{i(m)}\left(b_{m}\right)\right) \cdot \psi_{n+1}^{*}(\xi) .
$$

(3) Se $\xi \in E_{n}$, então $\psi_{n+1}^{*}(\xi)$ é tal que:

(a) $d\left(f_{i(n)}\left(b_{n}\right)\right) \cdot \overline{\psi_{n+1}^{*}(\xi)} \subset \psi_{n}^{*}(\xi)$;

(b) $\delta\left(\psi_{n+1}^{*}(\xi)\right)=\frac{r_{n+1}}{d(J) \cdot \prod_{m<n+1} d\left(f_{i(m)}\left(b_{m}\right)\right)}$.

Neste caso, façamos

$$
\psi_{n+1}(\xi)=d(J) \cdot \prod_{m<n+1} d\left(f_{i(m)}\left(b_{m}\right)\right) \cdot \psi_{n+1}^{*}(\xi) .
$$

(4) $\psi_{n}(i(n)) \cap \sum_{\mu \in \operatorname{supp} f_{i(n)}\left(b_{n}\right)} a\left(f_{i(n)}\left(b_{n}\right), \mu\right) \cdot d(J) \cdot \prod_{m<n} d\left(f_{i(m)}\left(b_{m}\right)\right) \cdot \psi_{n+1}^{*}(\mu) \neq \emptyset$.

Se $\xi \in E \backslash E_{n+1}$, façamos $\psi_{n+1}(\xi)=\mathbb{T}$. Se $\xi \in\left(E_{n+1} \backslash E_{n}\right) \backslash \operatorname{supp} f_{i(n)}\left(b_{n}\right)$, então $\psi_{n}(\xi)=\mathbb{T}$. Definimos $\psi_{n+1}^{*}(\xi)$ como sendo um elemento de $\mathcal{B}$ com diâmetro

$$
\delta\left(\psi_{n+1}^{*}(\xi)\right)=\frac{r_{n+1}}{d(J) \cdot \prod_{m<n+1} d\left(f_{i(m)}\left(b_{m}\right)\right)}
$$

e façamos

$$
\psi_{n+1}(\xi)=d(J) \cdot \prod_{m<n+1} d\left(f_{i(m)}\left(b_{m}\right)\right) \cdot \psi_{n+1}^{*}(\xi) .
$$

Se $\xi \in E_{n} \backslash \operatorname{supp} f_{i(n)}\left(b_{n}\right)$, fixemos uma raiz $d\left(f_{i(n)}\left(b_{n}\right)\right)$-ésima do ponto médio de $\psi_{n}^{*}(\xi)$. Definimos $\psi_{n+1}^{*}(\xi)$ como sendo o arco aberto de $\mathbb{T}$ centrado na raiz em questão com diâmetro

$$
\delta\left(\psi_{n+1}^{*}(\xi)\right)=\frac{r_{n+1}}{d(J) \cdot \prod_{m<n+1} d\left(f_{i(m)}\left(b_{m}\right)\right)}
$$

e façamos

$$
\psi_{n+1}(\xi)=d(J) \cdot \prod_{m<n+1} d\left(f_{i(m)}\left(b_{m}\right)\right) \cdot \psi_{n+1}^{*}(\xi)
$$


Vamos, agora, definir $\psi_{n+1}^{*}(\xi)$ e $\psi_{n+1}(\xi)$ para $\xi \in \operatorname{supp} f_{i(n)}\left(b_{n}\right)$.

Caso 1: $f_{i(n)}$ é do tipo 1 .

Tomemos $\alpha \in \operatorname{supp} f_{i(n)}\left(b_{n}\right) \backslash E_{n}$. Aplicando o lema 3.2 .3 para $\mathfrak{c}, d(J) \cdot \prod_{m<n} d\left(f_{i(m)}\left(b_{m}\right)\right)$, $r_{n}, \psi_{n}(i(n)), E_{n} \cap \operatorname{supp} f_{i(n)}\left(b_{n}\right), f_{i(n)}\left(b_{n}\right), \alpha$ e $\psi_{n}^{*}$, obtemos $\tilde{\psi}: \operatorname{supp} f_{i(n)}\left(b_{n}\right) \rightarrow \mathbb{T}$ satisfazendo as condições (i), (ii), (iii) e (iv) do lema 3.2.3. Façamos $\psi_{n+1}^{*}(\xi)=\tilde{\psi}(\xi)$, para todo $\xi \in \operatorname{supp} f_{i(n)}\left(b_{n}\right)$. As condições $(1),(2),(3)$ e (4) estão satisfeitas.

Caso 2: $f_{i(n)}$ é do tipo 2 .

Tomemos $\alpha \in \operatorname{supp} f_{i(n)}\left(b_{n}\right)$ tal que $q\left(f_{i(n)}\left(b_{n}\right), \alpha\right) \cdot r_{n}>d(J) \cdot \prod_{m<n} d\left(f_{i(m)}\left(b_{m}\right)\right)$. Podemos supor que $\alpha \in E_{n}$ pois, do contrário, os argumentos utilizados no caso 1 podem ser repetidos aqui. Aplicando o lema 3.2 .4 para $\mathfrak{c}, d(J) \cdot \prod_{m<n} d\left(f_{i(m)}\left(b_{m}\right)\right), r_{n}, \psi_{n}(i(n))$, $E_{n} \cap \operatorname{supp} f_{i(n)}\left(b_{n}\right), f_{i(n)}\left(b_{n}\right), \alpha$ e $\psi_{n}^{*}$, obtemos $\tilde{\psi}: \operatorname{supp} f_{i(n)}\left(b_{n}\right) \rightarrow \mathbb{T}$ satisfazendo as condições (i), (ii), (iii) e (iv) do lema 3.2.4. Façamos $\psi_{n+1}^{*}(\xi)=\tilde{\psi}(\xi)$, para todo $\xi \in \operatorname{supp} f_{i(n)}\left(b_{n}\right)$. As condições (1), (2), (3) e (4) estão satisfeitas.

Caso 3: $f_{i(n)}$ é do tipo 3 .

Tomemos $\alpha \in \operatorname{supp} f_{i(n)}\left(b_{n}\right)$ tal que $\left|a\left(f_{i(n)}\left(b_{n}\right), \alpha\right)\right| \cdot r_{n}>4 \cdot d\left(f_{i(n)}\left(b_{n}\right)\right)$. Novamente, podemos supor que $\alpha \in E_{n}$. Aplicando o lema 3.2 .5 para $\mathfrak{c}, d(J) \cdot \prod_{m<n} d\left(f_{i(m)}\left(b_{m}\right)\right), r_{n}$, $\psi_{n}(i(n)), E_{n} \cap \operatorname{supp} f_{i(n)}\left(b_{n}\right), f_{i(n)}\left(b_{n}\right), \alpha$ e $\psi_{n}^{*}$, obtemos $\tilde{\psi}: \operatorname{supp} f_{i(n)}\left(b_{n}\right) \rightarrow \mathbb{T}$ satisfazendo as condições (i), (ii), (iii) e (iv) do lema 3.2.5. Façamos $\psi_{n+1}^{*}(\xi)=\tilde{\psi}(\xi)$, para todo $\xi \in \operatorname{supp} f_{i(n)}\left(b_{n}\right)$. As condições $(1),(2),(3)$ e (4) estão satisfeitas.

Por indução, obtemos aplicações $\psi_{n}: E \rightarrow \mathcal{B}$ e $\psi_{n}^{*}: E_{n} \rightarrow \mathcal{B}$ para cada $n \in \omega$, satisfazendo as seguintes condições:

- $\overline{\psi_{n+1}(\xi)} \subset \psi_{n}(\xi)$, para todo $\xi \in E$;

- $\delta\left(\psi_{n}(\xi)\right)=r_{n}$, se $\xi \in E_{n}$ e $\psi_{n}(\xi)=\mathbb{T}$, se $\xi \in E \backslash E_{n}$;

- $\psi_{n}(\xi)=d(J) \cdot \prod_{m<n} d\left(f_{i(m)}\left(b_{m}\right)\right) \cdot \psi_{n}^{*}(\xi)$, se $\xi \in E_{n}$.

Como $\mathbb{T}$ é um espaço métrico completo e $\left(r_{n}\right)_{n \in \omega}$ é uma seqüência de números reais positivos que converge para 0 , concluímos que se $\xi \in E$, então $\cap_{n \in \omega} \psi_{n}(\xi)=\cap_{n \in \omega} \overline{\psi_{n}(\xi)}$ é um conjunto unitário. Denotaremos por $\phi\left(\chi_{\xi}\right)$ o único elemento de $\cap_{n \in \omega} \psi_{n}(\xi)$.

Para cada $\xi \in E$, sejam $N_{\xi}=\min \left\{n \in \omega: \xi \in E_{n}\right\}$ e $n \geq N_{\xi}$. Temos que $\psi_{n}(\xi) \neq \mathbb{T}$ e, portanto, existe um único elemento de $\psi_{n}^{*}(\xi)$ que multiplicado por $d(J) \cdot \prod_{m<n} d\left(f_{i(m)}\left(b_{m}\right)\right)$ 
é igual a $\phi\left(\chi_{\xi}\right)$. De fato, $\psi_{n}(\xi)=d(J) \cdot \prod_{m<n} d\left(f_{i(m)}\left(b_{m}\right)\right) \cdot \psi_{n}^{*}(\xi)$. Como $\phi\left(\chi_{\xi}\right) \in \psi_{n}(\xi)$, existe pelo menos um elemento de $\psi_{n}^{*}(\xi)$ que multiplicado por $d(J) \cdot \prod_{m<n} d\left(f_{i(m)}\left(b_{m}\right)\right)$ é igual a $\phi\left(\chi_{\xi}\right)$. Se $d(J) \cdot \prod_{m<n} d\left(f_{i(m)}\left(b_{m}\right)\right)=1$, nada temos a fazer. Suponhamos, portanto, que $d(J) \cdot \prod_{m<n} d\left(f_{i(m)}\left(b_{m}\right)\right)>1$. A distância entre duas raízes $d(J) \cdot \prod_{m<n} d\left(f_{i(m)}\left(b_{m}\right)\right)$-ésimas consecutivas de $\phi\left(\chi_{\xi}\right)$ é igual a

$$
\frac{1}{d(J) \cdot \prod_{m<n} d\left(f_{i(m)}\left(b_{m}\right)\right)} .
$$

Como $\psi_{n}^{*}(\xi)$ contém uma raiz $d(J) \cdot \prod_{m<n} d\left(f_{i(m)}\left(b_{m}\right)\right)$-ésima de $\phi\left(\chi_{\xi}\right)$ e

$$
\delta\left(\psi_{n}^{*}(\xi)\right)=\frac{r_{n}}{d(J) \cdot \prod_{m<n} d\left(f_{i(m)}\left(b_{m}\right)\right)}<\frac{1}{d(J) \cdot \prod_{m<n} d\left(f_{i(m)}\left(b_{m}\right)\right)}
$$

concluímos que $\psi_{n}^{*}(\xi)$ contém uma única raiz $d(J) \cdot \prod_{m<n} d\left(f_{i(m)}\left(b_{m}\right)\right)$-ésima de $\phi\left(\chi_{\xi}\right)$, ou seja, que existe um único elemento de $\psi_{n}^{*}(\xi)$ que multiplicado por $d(J) \cdot \prod_{m<n} d\left(f_{i(m)}\left(b_{m}\right)\right)$ é igual a $\phi\left(\chi_{\xi}\right)$. Denotaremos tal elemento por

$$
\phi\left(\frac{1}{d(J) \cdot \prod_{m<n} d\left(f_{i(m)}\left(b_{m}\right)\right)} \cdot \chi_{\xi}\right) .
$$

Consideremos

$$
G_{\xi}=\left\{\frac{1}{d(J) \cdot \prod_{m<n} d\left(f_{i(m)}\left(b_{m}\right)\right)} \cdot \chi_{\xi} \in \mathbb{Q}^{(E)}: n \geq N_{\xi}\right\}
$$

e denotemos por $G$ o grupo gerado por $\cup_{\xi \in E} G_{\xi}$. Uma vez que se $\xi \in E$ e $n>N_{\xi}$, então

$$
\phi\left(\frac{1}{d(J) \cdot \prod_{m<N_{\xi}} d\left(f_{i(m)}\left(b_{m}\right)\right)} \cdot \chi_{\xi}\right)=\prod_{N_{\xi} \leq m<n} d\left(f_{i(m)}\left(b_{m}\right)\right) \cdot \phi\left(\frac{1}{d(J) \cdot \prod_{m<n} d\left(f_{i(m)}\left(b_{m}\right)\right)} \cdot \chi_{\xi}\right)
$$

podemos estender $\phi$ a um homomorfismo de grupos de $G$ em $\mathbb{T}$. Usando o fato de $\mathbb{T}$ ser um grupo divisível, estendemos $\phi$ a um homomorfismo de grupos $\phi_{J} \uparrow_{\mathbb{Q}^{(E)}}: \mathbb{Q}^{(E)} \rightarrow \mathbb{T}$.

Temos que

$$
\phi_{J} \uparrow_{\mathbb{Q}^{(E)}}(J)=\sum_{\mu \in \operatorname{supp} J} a(J, \mu) \cdot \phi_{J} \uparrow_{\mathbb{Q}^{(E)}}\left(\frac{1}{d(J)} \cdot \chi_{\mu}\right) \in \sum_{\mu \in \operatorname{supp} J} a(J, \mu) \cdot \psi_{0}^{*}(\mu)
$$

e, portanto, $\phi_{J} \uparrow_{\mathbb{Q}^{(E)}}(J) \neq 0+\mathbb{Z}$. Logo, (i) está verificada. 
Fixemos $\xi \in E \backslash\{0\}$. Para cada $k \in i^{-1}(\{\xi\})$ temos que

$$
\phi_{J} \uparrow_{\mathbb{Q}^{(E)}}\left(f_{i(k)}\left(b_{k}\right)\right) \in \sum_{\mu \in \operatorname{supp} f_{i(k)}\left(b_{k}\right)} a\left(f_{i(k)}\left(b_{k}\right), \mu\right) \cdot d(J) \cdot \prod_{m<k} d\left(f_{i(m)}\left(b_{m}\right)\right) \cdot \psi_{k+1}^{*}(\mu) .
$$

Além disso,

$$
\phi_{J}\left\lceil_{\mathbb{Q}^{(E)}}\left(\chi_{i(k)}\right) \in \psi_{k}(i(k)) .\right.
$$

De (4) vem que

$$
\begin{aligned}
\delta\left(\phi_{J} \Gamma_{\mathbb{Q}^{(E)}}\left(f_{i(k)}\left(b_{k}\right)\right), \phi_{J} \Gamma_{\mathbb{Q}^{(E)}}\left(\chi_{\xi}\right)\right) & \leq d_{1}+d_{2} \\
& <2 r_{k}
\end{aligned}
$$

onde

$$
d_{1}=\delta\left(\sum_{\mu \in \operatorname{supp} f_{i(k)}\left(b_{k}\right)} a\left(f_{i(k)}\left(b_{k}\right), \mu\right) \cdot d(J) \cdot \prod_{m<k} d\left(f_{i(m)}\left(b_{m}\right)\right) \cdot \psi_{k+1}^{*}(\mu)\right)
$$

e

$$
d_{2}=\delta\left(\psi_{k}(i(k))\right)
$$

Como $r_{k} \rightarrow 0$, concluímos que a seqüência $\left\{\phi_{J} \Upsilon_{\mathbb{Q}^{(E)}}\left(f_{i(k)}\left(b_{k}\right)\right): k \in i^{-1}(\{\xi\})\right\}$ converge para $\phi_{J} \Gamma_{\mathbb{Q}^{(E)}}\left(\chi_{\xi}\right)$. Da propriedade (v) do lema 3.2.2 segue que

$$
\phi_{J} \uparrow_{\mathbb{Q}^{(E)}}\left(\chi_{\xi}\right)=p_{\xi}-\lim \left\{\phi_{J} \uparrow_{\mathbb{Q}^{(E)}}\left(f_{\xi}(n)\right): n \in \omega\right\} .
$$

Logo, (ii) está verificada.

Estamos prontos para estender $\phi_{J} \uparrow_{\mathbb{Q}^{(E)}}$ a um homomorfismo de grupos $\phi_{J}: \mathbb{Q}^{(\mathfrak{c})} \rightarrow \mathbb{T}$. Nossa única preocupação será garantir que $\phi_{J}\left(\chi_{\xi}\right)=p_{\xi}-\lim \left\{\phi_{J}\left(f_{\xi}(n)\right): n \in \omega\right\}$ para todo $\xi \in \mathfrak{c} \backslash E$, uma vez que supp $J \subset E$.

Lema 3.2.7. Para cada $J \in \mathbb{Q}^{(\mathfrak{c})} \backslash\{0\}$, existe $\phi_{J}: \mathbb{Q}^{(\mathfrak{c})} \rightarrow \mathbb{T}$ um homomorfismo de grupos tal que:

(i) $\phi_{J}(J) \neq 0+\mathbb{Z}$;

(ii) $\phi_{J}\left(\chi_{\xi}\right)=p_{\xi}-\lim \left\{\phi_{J}\left(f_{\xi}(n)\right): n \in \omega\right\}$, para cada $\left.\xi \in\right] 0, \mathfrak{c}[$.

Demonstração. Faremos a construção de $\phi_{J}$ por indução. De acordo com a proposição 3.2.1, existe $E \in[\mathfrak{c}]^{\omega}$ tal que supp $J \subset E$ e $\cup_{n \in \omega} \operatorname{supp} f_{\xi}(n) \subset E$, para cada $\xi \in E \backslash\{0\}$. Segundo o lema 3.2.6, é possível construir $\phi_{J} \uparrow_{\mathbb{Q}^{(E)}}: \mathbb{Q}^{(E)} \rightarrow \mathbb{T}$ um homomorfismo de grupos com as seguintes propriedades: 
(1) $\phi_{J} \uparrow_{\mathbb{Q}^{(E)}}(J) \neq 0+\mathbb{Z}$

(2) $\phi_{J} \Gamma_{\mathbb{Q}^{(E)}}\left(\chi_{\xi}\right)=p_{\xi}-\lim \left\{\phi_{J} \Gamma_{\mathbb{Q}^{(E)}}\left(f_{\xi}(n)\right): n \in \omega\right\}$, para cada $\xi \in E \backslash\{0\}$.

Consideremos $\left\{\alpha_{\xi}: \xi<\mathfrak{c}\right\}$ uma indexação estritamente crescente de $\mathfrak{c} \backslash E$. Definamos

$$
\tilde{\phi}_{J}\left(\chi_{\alpha_{0}}\right)=p_{\alpha_{0}}-\lim \left\{\phi_{J} \Upsilon_{\mathbb{Q}^{(E)}}\left(f_{\alpha_{0}}(n)\right): n \in \omega\right\}
$$

e façamos

$$
\tilde{\phi}_{J}(\tilde{J})=\phi_{J} \uparrow_{\mathbb{Q}^{(E)}}(\tilde{J})
$$

para todo $\tilde{J} \in \mathbb{Q}^{(E)} .^{2}$

Seja $G_{\alpha_{0}}$ o subgrupo de $\mathbb{Q}^{(\mathfrak{c})}$ gerado por $\mathbb{Q}^{(E)} \cup\left\{\chi_{\alpha_{0}}\right\}$. Podemos estender $\tilde{\phi}_{J}$ a um homomorfismo de grupos de $G_{\alpha_{0}}$ em $\mathbb{T}$. Uma vez que $\mathbb{T}$ é divisível e $G_{\alpha_{0}}$ é um subgrupo de $\mathbb{Q}^{\left(E \cup\left\{\alpha_{0}\right\}\right)}$, estendemos $\tilde{\phi}_{J}$ a um homomorfismo de grupos $\phi_{J} \uparrow_{\mathbb{Q}^{\left(E \cup\left\{\alpha_{0}\right\}\right)}}: \mathbb{Q}^{\left(E \cup\left\{\alpha_{0}\right\}\right)} \rightarrow \mathbb{T}$.

Repetindo indutivamente esta construção, obteremos $\phi_{J}: \mathbb{Q}^{(\mathfrak{c})} \rightarrow \mathbb{T}$ um homomorfismo de grupos satisfazendo (i) e (ii).

\subsection{Explicitando a topologia}

A próxima proposição mostra que o produto diagonal da família $\left\{\phi_{J}: J \in \mathbb{Q}^{(\mathfrak{c})} \backslash\{0\}\right\}$ é um monomorfismo de grupos.

Proposição 3.3.1. Suponhamos que para cada $J \in \mathbb{Q}^{(\mathfrak{c})} \backslash\{0\}$ exista um homomorfismo de grupos $\phi_{J}: \mathbb{Q}^{(\mathfrak{c})} \rightarrow \mathbb{T}$ tal que $\phi_{J}(J) \neq 0+\mathbb{Z}$. A aplicação

$$
\begin{aligned}
\Phi: \mathbb{Q}^{(\mathfrak{c})} & \rightarrow \mathbb{T}^{\mathbb{Q}^{(\mathfrak{c})} \backslash\{0\}} \\
\tilde{J} & \mapsto \Phi(\tilde{J})
\end{aligned}
$$

dada por

$$
\Phi(\tilde{J})(J)=\phi_{J}(\tilde{J}), \text { para cada } J \in \mathbb{Q}^{(\mathfrak{c})} \backslash\{0\}
$$

é um monomorfismo de grupos.

Demonstração. Análoga à da proposição 2.3.1.

\footnotetext{
${ }^{2}$ Estamos considerando $\mathbb{Q}^{(E)} \subset \mathbb{Q}^{\left(E \cup\left\{\alpha_{0}\right\}\right)} \subset \mathbb{Q}^{(\mathfrak{c})}$.
} 
Teorema 3.3.2. O grupo aditivo da reta real admite uma topologia que o torna um grupo topológico enumeravelmente compacto sem seqüências não triviais convergentes.

Demonstração. Temos que $\Phi\left[\mathbb{Q}^{(\mathfrak{c})}\right]$ munido da topologia de subespaço induzida por $\mathbb{T}^{\mathbb{Q}^{(\mathfrak{c})} \backslash\{0\}}$ é um grupo topológico. De acordo com as proposições 3.1.1 e 3.3.1, basta mostrar que $\Phi\left[\mathbb{Q}^{(\mathfrak{c})}\right]$ é enumeravelmente compacto e não possui seqüências não triviais convergentes.

Consideremos $f: \omega \rightarrow \Phi\left[\mathbb{Q}^{(\mathfrak{c})}\right]$. Se $f$ é trivial, nada temos a fazer. Se não, $f$ possui duas subseqüências constantes e distintas ou existe $j: \omega \rightarrow \omega$ estritamente crescente tal que $f \circ j$ é injetora. Com efeito, $\Phi^{-1} \circ f: \omega \rightarrow \mathbb{Q}^{(\mathfrak{c})}$ é não trivial. Se $\Phi^{-1} \circ f$ tem uma subseqüência do tipo $i$, para algum $i \in\{1,2,3\}$, então existe $j: \omega \rightarrow \omega$ estritamente crescente tal que $\Phi^{-1} \circ f \circ j$ é injetora. Suponhamos que $\Phi^{-1} \circ f$ não tenha subseqüências de qualquer um dos tipos acima mencionados. Existem, portanto, $j: \omega \rightarrow \omega$ estritamente crescente e $J \in \mathbb{Q}^{(\mathfrak{c})}$ tais que $\left(\Phi^{-1} \circ f \circ j\right)(n)=J$, para todo $n \in \omega$. Podemos supor que se $n \notin j[\omega]$, então $\left(\Phi^{-1} \circ f\right)(n) \neq J$. Como $\Phi^{-1} \circ f$ é não trivial, $\omega \backslash j[\omega]$ é um conjunto infinito. Aplicando novamente a proposição 3.1.3 e usando o fato de que $\Phi^{-1} \circ f$ não possui subseqüências dos tipos 1,2 ou 3, asseguramos a existência de uma outra subseqüência constante de $\Phi^{-1} \circ f$, distinta de $\Phi^{-1} \circ f \circ j$.

No primeiro caso, temos que $\left\{f(n) \in \Phi\left[\mathbb{Q}^{(\mathfrak{c})}\right]: n \in \omega\right\}$ tem pelo menos dois pontos de acumulação distintos e, portanto, $f$ não é convergente. Suponhamos que o segundo caso ocorra. As aplicações

$$
\begin{aligned}
g_{0}: \omega & \rightarrow \Phi\left[\mathbb{Q}^{(\mathfrak{c})}\right] \\
n & \mapsto(f \circ j)(2 n)
\end{aligned}
$$

e

$$
\begin{aligned}
g_{1}: \omega & \rightarrow \Phi\left[\mathbb{Q}^{(\mathfrak{c})}\right] \\
n & \mapsto(f \circ j)(2 n+1)
\end{aligned}
$$

são subseqüências distintas de $f$. De acordo com a proposição 3.1.3, existem $j_{0}, j_{1}: \omega \rightarrow \omega$ estritamente crescentes tais que $\Phi^{-1} \circ g_{0} \circ j_{0}, \Phi^{-1} \circ g_{1} \circ j_{1} \in \mathcal{F}$, uma vez que $f \circ j$ é injetora. Logo, existem $\left.\xi_{0}, \xi_{1} \in\right] 0, \mathfrak{c}\left[\right.$ distintos tais que $\Phi^{-1} \circ g_{0} \circ j_{0}=f_{\xi_{0}}$ e $\Phi^{-1} \circ g_{1} \circ j_{1}=f_{\xi_{1}}$.

Dado $i \in\{0,1\}$, temos que

$$
\phi_{J}\left(\chi_{\xi_{i}}\right)=p_{\xi_{i}}-\lim \left\{\phi_{J}\left(f_{\xi_{i}}(n)\right): n \in \omega\right\}
$$

para todo $J \in \mathbb{Q}^{(\mathfrak{c})} \backslash\{0\}$ e, portanto,

$$
\Phi\left(\chi_{\xi_{i}}\right)=p_{\xi_{i}}-\lim \left\{\left(\Phi \circ f_{\xi_{i}}\right)(n): n \in \omega\right\} .
$$


Logo, $\Phi\left(\chi_{\xi_{1}}\right)$ e $\Phi\left(\chi_{\xi_{2}}\right)$ são pontos de acumulação distintos de $\{f(n): n \in \omega\}$. 

CAPÍTULO 4

\section{Grupos abelianos quase livres de torção que têm cardinalidade $\mathfrak{c}$ : topologias de grupo enumeravelmente compactas sem seqüências não triviais convergentes e topologias independentes}

Neste capítulo, assumiremos a existência de $\mathfrak{c}$ ultrafiltros seletivos dois a dois incomparáveis (segundo a ordem de Rudin-Keisler) para mostrar que um grupo abeliano quase livre de torção $G$ tal que $|G|=\mathfrak{c}$ admite uma topologia que o torna um grupo topológico enumeravelmente compacto sem seqüências não triviais convergentes.

Como queremos aproveitar as idéias desenvolvidas no capítulo 3, começaremos imergindo o grupo $G$ na soma direta $(\mathbb{Q} / \mathbb{Z})^{(\omega)} \oplus \mathbb{Q}^{([\omega, \mathfrak{c}[)}$. Para cada $(H, J) \in(\mathbb{Q} / \mathbb{Z})^{(\omega)} \oplus \mathbb{Q}^{([\omega, \mathfrak{c}[)} \backslash\{(0,0)\}$ construiremos $\phi_{(H, J)}:(\mathbb{Q} / \mathbb{Z})^{(\omega)} \oplus \mathbb{Q}^{([\omega, \mathfrak{c}[)} \rightarrow \mathbb{T}$ um homomorfismo de grupos de modo que o produto diagonal da família $\left\{\phi_{(H, J)}:(H, J) \in(\mathbb{Q} / \mathbb{Z})^{(\omega)} \oplus \mathbb{Q}^{([\omega, \mathfrak{c}[)} \backslash\{(0,0)\}\right\}$ seja injetor e que a topologia em $G$ gerada pela composta deste produto diagonal com a imersão em $(\mathbb{Q} / \mathbb{Z})^{(\omega)} \oplus \mathbb{Q}^{([\omega, \mathfrak{c}[)}$ tenha as propriedades desejadas.

Uma das novidades deste capítulo é que $G$ pode conter elementos de ordem finita. Todavia, o fato de $G$ ser um grupo abeliano quase livre de torção impedirá que os elementos de uma seqüência injetora em $G$ tenham suas ordens limitadas por um número natural $n$. Portanto, poderemos continuar atribuindo a tais seqüências pontos de acumulação de ordem infinita.

Destacamos que é possível adaptar a construção descrita acima para obter o seguinte 
resultado: se $G$ é um grupo abeliano quase livre de torção e existe uma topologia $\tau$ em $G$ tal que $|G|=|\tau|=\mathfrak{c}$, então é possível construir uma topologia $\sigma$ em $G$ que é independente de $\tau$ e que torna $G$ um grupo topológico. Estas adaptações foram incorporadas já no início deste capítulo, fazendo com que o texto ficasse ligeiramente diferente do padrão apresentado nos capítulos 2 e 3 .

\subsection{Uma escolha adequada de seqüências}

Seja $G$ um grupo abeliano quase livre de torção tal que $|G|=\mathfrak{c}$ e suponhamos que exista $\tau$ uma topologia $T_{1}$ em $G$ tal que $|\tau|=\mathfrak{c}{ }^{1}$ Denotemos por $\mathcal{F}$ a família de todos os subconjuntos fechados de $G$ que têm cardinalidade $\mathfrak{c}$ e consideremos $\left\{F_{\beta}: \beta<\mathfrak{c}\right\}$ uma indexação de $\mathcal{F}$ tal que $F_{0}=G$ e $F_{\beta} \neq G$, para todo $\left.\beta \in\right] 0, \mathfrak{c}[$.

Seja $\varphi_{1}: G \rightarrow(\mathbb{Q} / \mathbb{Z})^{(\omega)} \oplus \mathbb{Q}^{([\omega, \mathfrak{c}[)}$ um monomorfismo de grupos. ${ }^{2}$ Temos que

$$
\varphi_{1}\left[F_{\beta}\right]=\left\{\left(x_{\xi}^{\beta}, y_{\xi}^{\beta}\right) \in(\mathbb{Q} / \mathbb{Z})^{(\omega)} \oplus \mathbb{Q}^{([\omega, \mathfrak{c}[)}: \omega \leq \xi<\mathfrak{c}\right\}
$$

qualquer que seja $\beta<\mathfrak{c}$. Para cada $\beta \in] 0, \mathfrak{c}\left[\right.$, tomemos $x^{\beta} \in(\mathbb{Q} / \mathbb{Z})^{(\omega)}$ tal que $\left|Q_{\beta}\right|=\mathfrak{c}$, onde

$$
Q_{\beta}=\left\{\left(x_{\xi}^{\beta}, y_{\xi}^{\beta}\right) \in \varphi_{1}\left[F_{\beta}\right]: x_{\xi}^{\beta}=x^{\beta}\right\}
$$

Tomemos, ainda, $x_{0} \in(\mathbb{Q} / \mathbb{Z})^{(\omega)}$ de modo que $\left\{\left(x_{\xi}^{0}, y_{\xi}^{0}\right) \in \varphi_{1}[G]: x_{\xi}^{0}=x_{0}\right\}$ tenha cardinalidade c. Se $x_{0}=0$, façamos $x^{0}=x_{0}$ e

$$
Q_{0}=\left\{\left(x_{\xi}^{0}, y_{\xi}^{0}\right) \in \varphi_{1}[G]: x_{\xi}^{0}=x^{0}\right\}
$$

Se não, existe $n>1$ um número natural tal que o $\left(x_{0}\right)=n$. Neste caso, definimos

$$
Q_{0}=\left\{\left(n x_{\xi}^{0}, n y_{\xi}^{0}\right) \in \varphi_{1}[G]: x_{\xi}^{0}=x_{0}\right\}
$$

e $x^{0}=n x_{0}=0$. Não é difícil notar que $\left|Q_{0}\right|=\mathfrak{c}$. Para cada $\beta<\mathfrak{c}$, consideremos

$$
P_{\beta}=\left\{y \in \mathbb{Q}^{([\omega, \mathfrak{c}[)}:\left(x^{\beta}, y\right) \in Q_{\beta}\right\} .
$$

\footnotetext{
${ }^{1}$ Observamos que a topologia cofinita em $G$ é $T_{1}$.

${ }^{2}$ Como $G$ é um grupo abeliano, segue que $G$ é isomorfo a um subgrupo de um grupo divisível. Além disso, todo grupo divisível é isomorfo a uma soma direta de cópias de $\mathbb{Q}$ e de $\mathbb{Z}_{p}(\infty) \subset \mathbb{Q} / \mathbb{Z}$, para $p$ número primo. Uma vez que $|G|=\mathfrak{c}$ e que $|T(G)| \leq \omega$, bastam $\omega$ cópias de $\mathbb{Q} / \mathbb{Z}$ e $\mathfrak{c}$ cópias de $\mathbb{Q}$.
} 
Como $\left|P_{\beta}\right|=\mathfrak{c}$, existe $\left\{S_{\beta}: \beta<\mathfrak{c}\right\}$ tal que:

- $S_{\beta} \subset P_{\beta}$, para cada $\beta<\mathfrak{c}$;

- $\left|S_{\beta}\right|=\mathfrak{c}$, para cada $\beta<\mathfrak{c}$;

- $S_{\alpha} \cap S_{\beta}=\emptyset$, quaisquer que sejam $\alpha, \beta<\mathfrak{c}$ distintos. $^{3}$

Tomemos $Z=\left\{z_{\xi}: \omega \leq \xi<\mathfrak{c}\right\}$ uma base de $\mathbb{Q}^{([\omega, \mathfrak{c}[)}$ como $\mathbb{Q}$-espaço vetorial tal que $\left|Z \cap S_{\beta}\right|=\mathfrak{c}$, para todo $\beta<\mathfrak{c}^{4}$ Consideremos $\varphi_{2}:(\mathbb{Q} / \mathbb{Z})^{(\omega)} \oplus \mathbb{Q}^{([\omega, \mathfrak{c}[)} \rightarrow(\mathbb{Q} / \mathbb{Z})^{(\omega)} \oplus \mathbb{Q}^{([\omega, \mathfrak{c}[)}$ o isomorfismo de grupos dado por $\varphi_{2}(a, 0)=(a, 0)$ e $\varphi_{2}\left(0, z_{\xi}\right)=\left(0, \chi_{\xi}\right)$, quaisquer que sejam $(a, 0) \in(\mathbb{Q} / \mathbb{Z})^{(\omega)} \oplus \mathbb{Q}^{([\omega, \mathfrak{c}[)}$ e $\xi \in\left[\omega, \mathfrak{c}\left[\right.\right.$, onde $\chi_{\xi}:[\omega, \mathfrak{c}[\rightarrow \mathbb{Q}$ é tal que

$$
\chi_{\xi}(\mu)=\left\{\begin{array}{lll}
1 & \text { se } & \mu=\xi \\
0 & \text { se } & \mu \neq \xi
\end{array}\right.
$$

e façamos $\varphi=\varphi_{2} \circ \varphi_{1}$.

Dado $\beta<\mathfrak{c}$, seja

$$
T_{\beta}=\left\{(x, y) \in Q_{\beta}: y \in S_{\beta} \cap Z\right\} .
$$

Temos que $\left|T_{\beta}\right|=\mathfrak{c}$ e $T_{\alpha} \cap T_{\beta}=\emptyset$, quaisquer que sejam $\alpha, \beta<\mathfrak{c}$ distintos. Consideremos, ainda,

$$
L_{\beta}=\left\{\xi \in \left[\omega, \mathfrak{c}\left[:\left(x^{\beta}, \chi_{\xi}\right) \in \varphi_{2}\left[T_{\beta}\right]\right\}\right.\right.
$$

Observamos que $\left|L_{\beta}\right|=\mathfrak{c}$ e que $L_{\alpha} \cap L_{\beta}=\emptyset$, quaisquer que sejam $\alpha, \beta<\mathfrak{c}$ distintos. Além disso,

$$
\left\{\left(0, \chi_{\xi}\right) \in(\mathbb{Q} / \mathbb{Z})^{(\omega)} \oplus \mathbb{Q}^{([\omega, \mathfrak{c}[)}: \xi \in L_{0}\right\} \subset \varphi[G]
$$

\footnotetext{
${ }^{3}$ Para cada $\beta<\mathfrak{c}$, consideremos uma partição $\left\{P_{\beta, \alpha}: \alpha<\mathfrak{c}\right\}$ de $P_{\beta}$ tal que $\left|P_{\beta, \alpha}\right|=\mathfrak{c}$, para todo $\alpha<\mathfrak{c}$. Tomemos $f: \mathfrak{c} \times \mathfrak{c} \rightarrow \mathfrak{c}$ uma bijeção tal que $f(0,0)=0$ e ordenemos $\mathfrak{c} \times \mathfrak{c}$ da seguinte maneira: $(\delta, \gamma) \prec(\beta, \alpha)$ se, e somente se, $f(\delta, \gamma)<f(\beta, \alpha)$. Fixemos $x_{0,0} \in P_{0,0}$. Seja $(\beta, \alpha) \in \mathfrak{c} \times \mathfrak{c}$ e suponhamos escolhidos $x_{\delta, \gamma} \in P_{\delta, \gamma}$, para todo $(\delta, \gamma) \prec(\alpha, \beta)$. Temos que $\left|\left\{x_{\delta, \gamma}:(\delta, \gamma) \prec(\alpha, \beta)\right\}\right|<\mathfrak{c}$, uma vez que $f(\alpha, \beta)<\mathfrak{c}$. Logo, existe $x_{\beta, \alpha} \in P_{\beta, \alpha} \backslash\left\{x_{\delta, \gamma}:(\delta, \gamma) \prec(\alpha, \beta)\right\}$. Dado $\beta<\mathfrak{c}$, denotemos $\left\{x_{\beta, \alpha}: \alpha<\mathfrak{c}\right\}$ por $S_{\beta}$.

${ }^{4}$ Consideremos $\mathcal{S}=\left\{S_{\beta}: \beta<\mathfrak{c}\right\}$ e tomemos $\left\{R_{\delta}: \delta<\mathfrak{c}\right\}$ uma indexação de $\mathcal{S}$ tal que, para cada $S \in \mathcal{S}$, o conjunto $\left\{\delta<\mathfrak{c}: S=R_{\delta}\right\}$ tenha cardinalidade $\mathfrak{c}$. Fixemos $x_{0} \in R_{0} \backslash\{0\}$. Seja $\alpha<\mathfrak{c}$ um ordinal e suponhamos escolhido, para cada $\gamma<\alpha, x_{\gamma} \in R_{\gamma}$ de modo que $\left\{x_{\gamma}: \gamma<\alpha\right\}$ seja um subconjunto linearmente independente do $\mathbb{Q}$-espaço vetorial $\mathbb{Q}^{([\omega, \mathfrak{c}[)}$. Como $\left|\left\langle\left\{x_{\gamma}: \gamma<\alpha\right\}\right\rangle\right| \leq \max \{\omega,|\alpha|\}<\mathfrak{c}$ e $\left|R_{\alpha}\right|=\mathfrak{c}$, existe $x_{\alpha} \in R_{\alpha}$ tal que $\left\{x_{\gamma}: \gamma<\alpha+1\right\}$ é linearmente independente. Por indução, obtemos $\left\{x_{\alpha}: \alpha<\mathfrak{c}\right\} \subset \mathbb{Q}^{([\omega, \mathfrak{c}[)}$ linearmente independente tal que $\left|\left\{x_{\alpha}: \alpha<\mathfrak{c}\right\} \cap S_{\beta}\right|=\mathfrak{c}$, para todo $\beta<\mathfrak{c}$. Seja $B$ uma base de $\mathbb{Q}^{([\omega, \mathfrak{c}[)}$ que contém $\left\{x_{\alpha}: \alpha<\mathfrak{c}\right\}$. Como $|B|=\mathfrak{c}$, podemos tomar $\left\{z_{\xi}: \omega \leq \xi<\mathfrak{c}\right\}$ uma indexação de $B$.
} 
Se $(H, J) \in(\mathbb{Q} / \mathbb{Z})^{(\omega)} \oplus \mathbb{Q}^{([\omega, \mathfrak{c}[)}$, então

$$
H(n)=\frac{p(H, n)}{q(H, n)}+\mathbb{Z}
$$

onde $p(H, n), q(H, n) \in \mathbb{Z}, q(H, n)>0,0 \leq p(H, n) \leq q(H, n)-1$ e $\operatorname{mdc}(p(H, n), q(H, n))=1$, para cada $n \in \operatorname{supp} H$. Além disso,

$$
J(\mu)=\frac{p(J, \mu)}{q(J, \mu)}
$$

onde $p(J, \mu), q(J, \mu) \in \mathbb{Z}, q(J, \mu)>0$ e $\operatorname{mdc}(p(J, \mu), q(J, \mu))=1$, para cada $\mu \in \operatorname{supp} J$. Sejam

$$
\begin{gathered}
|q(H)|=\max \{q(H, n): n \in \operatorname{supp} H\}, \\
|q(J)|=\max \{q(J, \mu): \mu \in \operatorname{supp} J\}
\end{gathered}
$$

e

$$
|p(J)|=\max \{|p(J, \mu)|: \mu \in \operatorname{supp} J\} .
$$

Consideremos

$$
d(H)=\operatorname{mmc}\{q(H, n): n \in \operatorname{supp} H\}
$$

e

$$
d(J)=\operatorname{mmc}\{q(J, \mu): \mu \in \operatorname{supp} J\} .^{5}
$$

Para cada $n \in \operatorname{supp} H$, seja

$$
a(H, n)=p(H, n) \cdot \frac{d(H)}{q(H, n)}
$$

e, para cada $\mu \in \operatorname{supp} J, \operatorname{seja}$

$$
a(J, \mu)=p(J, \mu) \cdot \frac{d(J)}{q(J, \mu)} .
$$

Sejam, ainda,

$$
|a(J)|=\max \{|a(J, \mu)|: \mu \in \operatorname{supp} J\}
$$

e

$$
|| a(H, J) \|=\sum_{n \in \operatorname{supp} H}|a(H, n)|+\sum_{\mu \in \operatorname{supp} J}|a(J, \mu)| .
$$

Por fim, observamos que $\operatorname{supp}(H, J)=\operatorname{supp} H \cup \operatorname{supp} J$.

\footnotetext{
${ }^{5}$ Se $H=0$, então $d(H)=1$. Analogamente, se $J=0$, então $d(J)=1$.
} 
Denotaremos por $\mathcal{H}$ o conjunto de todas as funções

$$
\begin{aligned}
h: \omega & \rightarrow \varphi[G] \subset(\mathbb{Q} / \mathbb{Z})^{(\omega)} \oplus \mathbb{Q}^{([\omega, \mathfrak{c}[)} \\
n & \mapsto(g(n), f(n))
\end{aligned}
$$

que satisfazem uma das seguintes condições:

(1) $\operatorname{supp} f(n) \backslash \cup_{m<n} \operatorname{supp} f(m) \neq \emptyset$, para todo $n \in \omega$;

(2) $|q(f(n))|>n$, para todo $n \in \omega$;

(3) $\{|q(f(n))|: n \in \omega\}$ é limitado e $|p(f(n))|>n$, para todo $n \in \omega$;

(4) $|q(g(n))|>n$, para todo $n \in \omega$.

Definição 4.1.1. Sejam $h \in \mathcal{H}$ e $i \in\{1,2,3,4\}$. Dizemos que $h$ é do tipo i se $h$ satisfaz a condição (i) descrita acima.

Proposição 4.1.2. Se $h: \omega \rightarrow \varphi[G] \subset(\mathbb{Q} / \mathbb{Z})^{(\omega)} \oplus \mathbb{Q}^{([\omega, \mathfrak{c}[)}$, então existe $j: \omega \rightarrow \omega$ estritamente crescente tal que $h \circ j$ é constante ou é de um dos tipos acima mencionados.

Demonstração. Para cada $n \in \omega$, escreveremos $h(n)=(g(n), f(n))$, onde $g: \omega \rightarrow(\mathbb{Q} / \mathbb{Z})^{(\omega)} \mathrm{e}$ $f: \omega \rightarrow \mathbb{Q}^{([\omega, \mathfrak{c}[) .6}$ Há cinco casos a serem considerados.

Caso 1: $\cup_{n \in \omega} \operatorname{supp} f(n)$ é infinito.

Seja $n_{0}=0$. Fixemos $k \in \omega$ e suponhamos definidos $n_{0}, n_{1}, \ldots, n_{k}$. Uma vez que $\cup_{n \in \omega} \operatorname{supp} f(n)$ é infinito, existe um número natural $n_{k+1}>n_{k}$ tal que $\operatorname{supp} f\left(n_{k+1}\right) \backslash$ $\cup_{l<k+1} \operatorname{supp} f\left(n_{l}\right) \neq \emptyset$. Temos que

$$
\begin{aligned}
j: \omega & \rightarrow \omega \\
k & \mapsto n_{k}
\end{aligned}
$$

é uma função estritamente crescente tal que $h \circ j$ é do tipo 1.

Caso 2: $\cup_{n \in \omega} \operatorname{supp} f(n)$ é finito e $\{|q(f(n))|: n \in \omega\}$ é ilimitado.

Por indução, tomemos $\left\{n_{k}: k \in \omega\right\}$ uma seqüência estritamente crescente de números

\footnotetext{
${ }^{6}$ Esta notação continuará sendo adotada ao longo deste capítulo.
} 
naturais tal que $\left|q\left(f\left(n_{k}\right)\right)\right|>k$, para todo $k \in \omega$. Temos que

$$
\begin{aligned}
j: \omega & \rightarrow \omega \\
k & \mapsto n_{k}
\end{aligned}
$$

é uma função estritamente crescente tal que $h \circ j$ é do tipo 2 .

Caso 3: $\cup_{n \in \omega} \operatorname{supp} f(n)$ é finito, $\{|q(f(n))|: n \in \omega\}$ é limitado e $\{|p(f(n))|: n \in \omega\}$ é ilimitado. Por indução, tomemos $\left\{n_{k}: k \in \omega\right\}$ uma seqüência estritamente crescente de números naturais tal que $\left|p\left(f\left(n_{k}\right)\right)\right|>k$, para todo $k \in \omega$. Temos que

$$
\begin{aligned}
j: \omega & \rightarrow \omega \\
k & \mapsto n_{k}
\end{aligned}
$$

é uma função estritamente crescente tal que $h \circ j$ é do tipo 3.

Caso 4: $\cup_{n \in \omega} \operatorname{supp} f(n)$ é finito, $\{|q(f(n))|: n \in \omega\}$ e $\{|p(f(n))|: n \in \omega\}$ são limitados e $\{|q(g(n))|: n \in \omega\}$ é ilimitado.

Por indução, tomemos $\left\{n_{k}: k \in \omega\right\}$ uma seqüência estritamente crescente de números naturais tal que $\left|q\left(g\left(n_{k}\right)\right)\right|>k$, para todo $k \in \omega$. Temos que

$$
\begin{aligned}
j: \omega & \rightarrow \omega \\
k & \mapsto n_{k}
\end{aligned}
$$

é uma função estritamente crescente tal que $h \circ j$ é do tipo 4 .

Caso 5: $\cup_{n \in \omega} \operatorname{supp} f(n)$ é finito, $\{|q(f(n))|: n \in \omega\},\{|p(f(n))|: n \in \omega\}$ e $\{|q(g(n))|: n \in \omega\}$ são limitados.

Existe $j_{1}: \omega \rightarrow \omega$ estritamente crescente tal que $f \circ j_{1}$ é constante. Existem, também, $m \in \omega$ e $j_{2}: \omega \rightarrow \omega$ estritamente crescente tais que $\left|q\left(g \circ j_{1} \circ j_{2}(n)\right)\right|=m$, para todo $n \in \omega$. Se $\cup_{n \in \omega} \operatorname{supp} g \circ j_{1} \circ j_{2}(n)$ é finito, então existe $j_{3}: \omega \rightarrow \omega$ estritamente crescente tal que $g \circ j_{1} \circ j_{2} \circ j_{3}$ é constante. Neste caso, temos que $h \circ j$ é constante, onde $j=j_{1} \circ j_{2} \circ j_{3}$. Se $\cup_{n \in \omega} \operatorname{supp} g \circ j_{1} \circ j_{2}(n)$ fosse infinito, existiria $j_{3}: \omega \rightarrow \omega$ estritamente crescente tal que $\operatorname{supp} g \circ j_{1} \circ j_{2} \circ j_{3}(n) \backslash \cup_{m<n} \operatorname{supp} g \circ j_{1} \circ j_{2} \circ j_{3}(m) \neq \emptyset$, para todo $n \in \omega$. Neste caso, $\left\{h \circ j_{1} \circ j_{2} \circ j_{3}(n): n \in \omega\right\} \not \subset \varphi[G]$ pois, do contrário, $\left\{h \circ j_{1} \circ j_{2} \circ j_{3}(n)-h \circ j_{1} \circ j_{2} \circ j_{3}(0):\right.$ $n \in \omega\}=\left\{\left(g \circ j_{1} \circ j_{2} \circ j_{3}(n)-g \circ j_{1} \circ j_{2} \circ j_{3}(0), 0\right): n \in \omega\right\}$ também estaria contido em $\varphi[G]$, o que é absurdo, uma vez que $\left\{\left(g \circ j_{1} \circ j_{2} \circ j_{3}(n)-g \circ j_{1} \circ j_{2} \circ j_{3}(0), 0\right): n \in \omega\right\} \subset G[m !]$ é 
infinito e $G$ é quase livre de torção.

Proposição 4.1.3. Existe $\left\{h_{\xi}: \xi \in L_{0}\right\}$ uma indexação de $\mathcal{H}$ tal que $\cup_{n \in \omega} \operatorname{supp} h_{\xi}(n) \subset \xi$, para todo $\xi \in L_{0}$.

Demonstração. Observamos, primeiramente, que $|\mathcal{H}|=\mathfrak{c}^{\omega}=\mathfrak{c}$. Portanto, podemos considerar $\left\{\tilde{h}_{\xi}: \xi \in L_{0}\right\}$ uma indexação de $\mathcal{H}$ tal que, para cada $h \in \mathcal{H}$, o conjunto

$$
A_{h}=\left\{\xi \in L_{0}: h=\tilde{h}_{\xi}\right\}
$$

tenha cardinalidade $\mathfrak{c}$.

Fixemos $h \in \mathcal{H}$. Como $\cup_{n \in \omega} \operatorname{supp} h(n) \subset \mathfrak{c}, \operatorname{cf}(\mathfrak{c})>\omega$ e $\left|\cup_{n \in \omega} \operatorname{supp} h(n)\right| \leq \omega$, existe $\alpha<\mathfrak{c}$ tal que $\cup_{n \in \omega} \operatorname{supp} h(n) \subset \alpha$. Seja $\xi \in A_{h}$. Se $\xi<\alpha$, façamos $h_{\xi}=h$, onde $h(n)=\left(g(n), 0_{\mathbb{Q}([\omega, \mathfrak{c}[)}\right)$, com $g(n)=\frac{1}{n+1} \cdot \chi_{0}$, para todo $n \in \omega$. Temos que $h$ é do tipo 4 e, portanto, $h \in \mathcal{H}$. Se $\xi \geq \alpha$, façamos $h_{\xi}=\tilde{h}_{\xi}$.

Vê-se facilmente que $\left\{h_{\xi}: \xi \in L_{0}\right\}$ é uma indexação de $\mathcal{H}$ tal que $\cup_{n \in \omega} \operatorname{supp} h_{\xi}(n) \subset \xi$, para todo $\xi \in L_{0}$.

\subsection{Construindo homomorfismos de grupos}

Sejam $\left\{p_{\xi}: \xi \in L_{0}\right\}$ uma família de ultrafiltros seletivos dois a dois incomparáveis segundo a ordem de Rudin-Keisler e $\left\{h_{\xi}: \xi \in L_{0}\right\}$ uma indexação de $\mathcal{H}$ tal que $\cup_{n \in \omega} \operatorname{supp} h_{\xi}(n) \subset \xi$, qualquer que seja $\xi \in L_{0}$.

Proposição 4.2.1. Seja $(H, J) \in(\mathbb{Q} / \mathbb{Z})^{(\omega)} \oplus \mathbb{Q}^{([\omega, \mathfrak{c}[)} \backslash\{(0,0)\}$. Existe $E \in[\mathfrak{c}]^{\omega}$ tal que:

(i) $\operatorname{supp}(H, J) \subset E$;

(ii) $\left|E \cap L_{0}\right|=\omega$;

(iii) $\cup_{n \in \omega} \operatorname{supp} h_{\xi}(n) \subset E$, qualquer que seja $\xi \in E \cap L_{0}$.

Demonstração. Para cada $\xi \in \mathfrak{c} \backslash L_{0}$, façamos $E(\xi)=\{\xi\} \cup \omega$. Se $\xi \in L_{0}$, definimos indutivamente

$$
E(\xi)=\{\xi\} \cup \bigcup_{\mu \in \cup_{n \in \omega} \operatorname{supp} h_{\xi}(n)} E(\mu) .
$$

Fixemos $\tilde{L}_{0} \in\left[L_{0}\right]^{\omega}$ e façamos

$$
E=\bigcup_{\zeta \in \operatorname{supp}(H, J) \cup \tilde{L}_{0}} E(\zeta)
$$


Temos que $\operatorname{supp}(H, J) \cup \tilde{L}_{0} \subset E$, uma vez que $\zeta \in E(\zeta) \subset E$, qualquer que seja $\zeta \in$ $\operatorname{supp}(H, J) \cup \tilde{L}_{0}$. Além disso, um argumento indutivo garante que $E(\xi) \in[\mathfrak{c}]^{\omega}$ para todo $\xi<\mathfrak{c}$ e, portanto, $E \in\left[\mathrm{cc}^{\omega}\right.$. Por fim, se $\xi \in E \cap L_{0}$, então existe $\zeta \in \operatorname{supp}(H, J) \cup \tilde{L}_{0}$ tal que $\xi \in E(\zeta)$. Um outro argumento indutivo garante que se $\alpha \in E(\beta)$, então $E(\alpha) \subset E(\beta)$, quaisquer que sejam $\alpha, \beta<\mathfrak{c} . \operatorname{Logo}, \cup_{n \in \omega} \operatorname{supp} h_{\xi}(n) \subset E(\xi) \subset E(\zeta) \subset E$.

Lema 4.2.2. Sejam $(H, J) \in(\mathbb{Q} / \mathbb{Z})^{(\omega)} \oplus \mathbb{Q}^{([\omega, \mathfrak{c}[)} \backslash\{(0,0)\}$ e $E \in[\mathfrak{c}]^{\omega}$ satisfazendo as condições (i), (ii) e (iii) da proposição 4.2.1. Existe $\left\{E_{k}: k \in \omega\right\}$ uma familia de subconjuntos finitos de $E,\left\{b_{k}: k \in \omega\right\}$ uma seqüência estritamente crescente de números naturais, $\left\{r_{k}: k \in \omega\right\}$ uma seqüência de números reais positivos e $i: \omega \rightarrow E \cap L_{0}$ tais que:

(i) $\operatorname{supp}(H, J) \subset E_{0}$;

(ii) $E=\cup_{k \in \omega} E_{k}$;

(iii) $i(k) \in E_{k}$, para todo $k \in \omega$;

(iv) $E_{k+1} \supset E_{k} \cup \bigcup\left\{\operatorname{supp} h_{i(m)}\left(b_{m}\right): m \leq k\right\}$, para todo $k \in \omega$;

(v) $\left\{b_{k}: k \in i^{-1}(\{\xi\})\right\} \in p_{\xi}$, para todo $\xi \in E \cap L_{0}$;

(vi) Se $h_{i(k)}$ é do tipo 1, então $\operatorname{supp} f_{i(k)}\left(b_{k}\right) \backslash E_{k} \neq \emptyset$;

(vii) Se $h_{i(k)}$ é do tipo 2, então $\left|q\left(f_{i(k)}\left(b_{k}\right)\right)\right| \cdot r_{k}>d(J) \cdot \prod_{m<k} d\left(f_{i(m)}\left(b_{m}\right)\right)$;

(viii) Se $h_{i(k)}$ é do tipo 3, então $\left|a\left(f_{i(k)}\left(b_{k}\right)\right)\right| \cdot r_{k}>4 \cdot d\left(f_{i(k)}\left(b_{k}\right)\right)$;

(ix) Se $h_{i(k)}$ é do tipo 4, então $\left|q\left(g_{i(k)}\left(b_{k}\right)\right)\right| \cdot r_{k}>d(H) \cdot \prod_{m<k} d\left(g_{i(m)}\left(b_{m}\right)\right)$;

(x) $r_{0}=\frac{1}{4 \cdot\|a(H, J)\|}$;

(xi) $r_{k+1}=\frac{r_{k}}{2 \cdot\left\|a\left(h_{i(k)}\left(b_{k}\right)\right)\right\|}$, para todo $k \in \omega$.

Demonstração. Consideremos $\left\{a_{n}: n \in \omega\right\}$ uma enumeração de $E$. Sejam $F_{0}=\operatorname{supp}(H, J) \cup\left\{a_{0}\right\}$ e $F_{n+1}=F_{n} \cup \bigcup\left\{\operatorname{supp} h_{\xi}(m): m \leq n, \xi \in F_{n} \cap L_{0}\right\} \cup\left\{a_{n+1}\right\}$, para cada $n \in \omega$. Temos que $\left\{F_{n}: n \in \omega\right\}$ é uma família de subconjuntos finitos de $E$ tal que:

(1) $\operatorname{supp}(H, J) \subset F_{0}$;

(2) $E=\cup_{n \in \omega} F_{n}$; 
(3) $F_{n+1} \supset F_{n} \cup \bigcup\left\{\operatorname{supp} h_{\xi}(m): m \leq n, \xi \in F_{n} \cap L_{0}\right\}$.

Fixemos $\xi \in E \cap L_{0}$ e $n \in \omega$. Se $h_{\xi}$ é do tipo 1 , definimos

$$
A_{n}^{\xi}=\left\{k \in \omega: \operatorname{supp} f_{\xi}(k) \backslash F_{n} \neq \emptyset\right\} .
$$

Se $h_{\xi}$ é do tipo 2 , definimos

$$
A_{n}^{\xi}=\left\{k \in \omega:\left|q\left(f_{\xi}(k)\right)\right|>2^{n+2} \cdot X_{n-1}\right\}
$$

onde

$$
X_{m}=\|a(H, J)\| \cdot d(J) \cdot \prod_{\substack{l \leq m \\ \zeta \in F_{m} \cap L_{0}}} d\left(f_{\zeta}(l)\right) \cdot \prod_{\substack{l \leq m \\ \zeta \in F_{m} \cap L_{0}}}\left\|a\left(h_{\zeta}(l)\right)\right\|
$$

para todo $m \in \omega \mathrm{e}$

$$
X_{-1}=\|a(H, J)\| \cdot d(J) .
$$

Se $h_{\xi}$ é do tipo 3, então $\left|a\left(f_{\xi}(n)\right)\right|>n$, para cada $n \in \omega$ e $\left\{\left|q\left(f_{\xi}(n)\right)\right|: n \in \omega\right\}$ é limitado. Tomemos $M_{\xi} \in \omega$ tal que $\left|q\left(f_{\xi}(n)\right)\right| \leq M_{\xi}$, para todo $n \in \omega$. Definimos

$$
A_{n}^{\xi}=\left\{k \in \omega:\left|a\left(f_{\xi}(k)\right)\right|>2^{n+4} \cdot M_{\xi} ! \cdot Y_{n-1}\right\}
$$

onde

$$
Y_{m}=\|a(H, J)\| \cdot \prod_{\substack{l \leq m \\ \zeta \in F_{m} \cap L_{0}}}\left\|a\left(h_{\zeta}(l)\right)\right\|
$$

para todo $m \in \omega \mathrm{e}$

$$
Y_{-1}=\|a(H, J)\|
$$

Se $h_{\xi}$ é do tipo 4, definimos

$$
A_{n}^{\xi}=\left\{k \in \omega:\left|q\left(g_{\xi}(k)\right)\right|>2^{n+2} \cdot Z_{n-1}\right\}
$$

onde

$$
Z_{m}=\|a(H, J)\| \cdot d(H) \cdot \prod_{\substack{l \leq m \\ \zeta \in F_{m} \cap L_{0}}} d\left(g_{\zeta}(l)\right) \cdot \prod_{\substack{l \leq m \\ \zeta \in F_{m} \cap L_{0}}}\left\|a\left(h_{\zeta}(l)\right)\right\|
$$


para todo $m \in \omega \mathrm{e}$

$$
Z_{-1}=\|a(H, J)\| \cdot d(H)
$$

Observamos que $A_{n}^{\xi}$ é um subconjunto cofinito de $\omega$, quaisquer que sejam $n \in \omega$ e $\xi \in E \cap L_{0}$. Logo, para cada $\xi \in E \cap L_{0}$, temos que $\left\{A_{n}^{\xi}: n \in \omega\right\} \subset p_{\xi}$, uma vez que $p_{\xi}$ é um ultrafiltro livre sobre $\omega$. Da seletividade de $p_{\xi}$ decorre que existe uma seqüência $\left\{a_{n}^{\xi}: n \in \omega\right\} \in p_{\xi}$ tal que $a_{n}^{\xi} \in A_{n}^{\xi}$ e $n<a_{n}^{\xi}$, para cada $n \in \omega$.

Do lema 1.5.4 segue que existe $\left\{I_{\xi}: \xi \in E \cap L_{0}\right\}$ uma família de subconjuntos de $\omega$ dois a dois disjuntos tal que:

(a) $\left\{a_{n}^{\xi}: n \in I_{\xi}\right\} \in p_{\xi}$, para cada $\xi \in E \cap L_{0}$;

(b) $\left\{\left[n, a_{n}^{\xi}\right]: n \in I_{\xi}, \xi \in E \cap L_{0}\right\}$ é uma família de intervalos de $\omega$ dois a dois disjuntos.

Para cada $\xi \in E \cap L_{0}$, seja

(c) $N_{\xi}=\min \left\{n \in \omega: \xi \in F_{n}\right\}$.

Podemos supor, sem perda de generalidade, que

(d) $N_{\xi}<n$, para todo $n \in I_{\xi}$.

Consideremos $\left\{n_{k}: k \in \omega\right\}$ uma enumeração estritamente crescente de $\dot{\cup}_{\xi \in E \cap L_{0}} I_{\xi}$. Seja

$$
\begin{aligned}
i: \omega & \rightarrow E \cap L_{0} \\
k & \mapsto i(k)
\end{aligned}
$$

onde $i(k)$ é o único elemento de $E \cap L_{0}$ tal que $n_{k} \in I_{i(k)}$.

Para cada $k \in \omega$, façamos $b_{k}=a_{n_{k}}^{i(k)}, E_{k}=F_{n_{k}}$ e definamos $r_{k}$ segundo os ítens (x) e (xi) do enunciado deste lema. Resta mostrar que as condições (i)-(ix) estão satisfeitas.

(i) Como $0 \leq n_{0}$, temos que $E_{0}=F_{n_{0}} \supset F_{0} \supset \operatorname{supp}(H, J)$.

(ii) $\cup_{k \in \omega} E_{k}=\cup_{k \in \omega} F_{n_{k}}=E$.

(iii) Seja $k \in \omega$. Temos que $n_{k} \in I_{i(k)}$. De (d) segue que $N_{i(k)}<n_{k}$. De (c) e (3) vem que $i(k) \in F_{N_{i(k)}} \subset F_{n_{k}}=E_{k}$. 
(iv) De (3) vem que $E_{k} \subset E_{k+1}$, pois $\left\{n_{k}: k \in \omega\right\}$ é uma enumeração estritamente crescente de $\cup_{\xi \in E \cap L_{0}} I_{\xi}$. Resta mostrar que $\operatorname{supp} h_{i(m)}\left(b_{m}\right) \subset E_{k+1}$, para cada $m \leq k$. De (3) decorre que

$$
E_{k+1}=F_{n_{k+1}} \supset \bigcup\left\{\operatorname{supp} h_{\xi}(m): m \leq n_{k+1}-1, \xi \in F_{n_{k+1}-1} \cap L_{0}\right\} .
$$

Por (iii), $i(m) \in E_{m} \subset E_{k}=F_{n_{k}} \subset F_{n_{k+1}-1}$, para todo $m \leq k$. Além disso, $i(m) \in L_{0}$. Por (b), $b_{m}=a_{n_{m}}^{i(m)} \leq n_{k+1}-1$, para todo $m \leq k$. Logo, $\operatorname{supp} h_{i(m)}\left(b_{m}\right) \subset E_{k+1}$.

(v) Seja $\xi \in E \cap L_{0}$. Temos que $\left\{b_{k}: k \in i^{-1}(\{\xi\})\right\}=\left\{a_{n_{k}}^{i(k)}: k \in i^{-1}(\{\xi\})\right\}=\left\{a_{n}^{\xi}: n \in I_{\xi}\right\} \in$ $p_{\xi}$, por (a).

(vi) Suponhamos que $h_{i(k)}$ seja do tipo 1. Temos que $b_{k}=a_{n_{k}}^{i(k)} \in A_{n_{k}}^{i(k)}$. Da definição de $A_{n_{k}}^{i(k)}$ vem que

$$
\operatorname{supp} f_{i(k)}\left(b_{k}\right) \backslash E_{k}=\operatorname{supp} f_{i(k)}\left(b_{k}\right) \backslash F_{n_{k}} \neq \emptyset \text {. }
$$

(vii) Suponhamos que $h_{i(k)}$ seja do tipo 2. Temos que $b_{k}=a_{n_{k}}^{i(k)} \in A_{n_{k}}^{i(k)}$. Da definição de $A_{n_{k}}^{i(k)}$ vem que

$$
\left|q\left(f_{i(k)}\left(b_{k}\right)\right)\right|>2^{n_{k}+2} \cdot X_{n_{k}-1}
$$

onde

$$
X_{n_{k}-1}=\|a(H, J)\| \cdot d(J) \cdot \prod_{\substack{m \leq n_{k}-1 \\ \xi \in F_{n_{k}-1} \cap L_{0}}} d\left(f_{\xi}(m)\right) \cdot \prod_{\substack{m \leq n_{k}-1 \\ \xi \in F_{n_{k}-1} \cap L_{0}}}\left\|a\left(h_{\xi}(m)\right)\right\| .
$$

Observamos que se $m<k$, então $n_{m} \leq n_{k-1}$ e, portanto, $i(m) \in E_{m}=F_{n_{m}} \subset F_{n_{k-1}} \subset$ $F_{n_{k}-1}$. Note que $i(m) \in L_{0}$. Como $b_{m}=a_{n_{m}}^{i(m)}$ e $\left[n_{m}, a_{n_{m}}^{i(m)}\right] \cap\left[n_{k}, a_{n_{k}}^{i(k)}\right]=\emptyset$, temos que $b_{m}<n_{k}$ e, portanto, $b_{m} \leq n_{k}-1$. Uma vez que

$$
r_{k}=\frac{1}{2^{k+2} \cdot\|a(H, J)\| \cdot \prod_{m<k}\left\|a\left(h_{i(m)}\left(b_{m}\right)\right)\right\|}
$$

concluímos que

$$
\left|q\left(f_{i(k)}\left(b_{k}\right)\right)\right| \cdot r_{k}>d(J) \cdot \prod_{m<k} d\left(f_{i(m)}\left(b_{m}\right)\right) .
$$

(viii) Suponhamos que $h_{i(k)}$ seja do tipo 3. Um argumento análogo ao apresentado em (vii) nos permite concluir que

$$
\left|a\left(f_{i(k)}\left(b_{k}\right)\right)\right| \cdot r_{k}>4 \cdot M_{i(k)} ! \geq 4 \cdot d\left(f_{i(k)}\left(b_{k}\right)\right) .
$$


(ix) Suponhamos que $h_{i(k)}$ seja do tipo 4. Um argumento análogo ao apresentado em (vii) nos permite concluir que

$$
\left|q\left(g_{i(k)}\left(b_{k}\right)\right)\right| \cdot r_{k}>d(H) \cdot \prod_{m<k} d\left(g_{i(m)}\left(b_{m}\right)\right) .
$$

O próximo lema compreende a parte técnica referente ao tipo 4.

Lema 4.2.3. Sejam $I$ um conjunto infinito, $c \in \mathbb{N} \backslash\{0\}, \epsilon>0, A \in \mathcal{B} \operatorname{com} \delta(A) \geq \epsilon$, $H \in(\mathbb{Q} / \mathbb{Z})^{(I)}, n \in \operatorname{supp} H \operatorname{com} q(H, n) \cdot \epsilon>c$ e $x \in \mathbb{T}$. Existe $y \in \mathbb{T}$ tal que $d(H) \cdot y=x$ e $a(H, n) \cdot c \cdot y \in A$.

Demonstração. Existem $d(H)$ raízes $d(H)$-ésimas de $x$. Se multiplicarmos cada uma delas por $a(H, n) \cdot c$ teremos

$$
\frac{q(H, n)}{\operatorname{mdc}(c, q(H, n))}
$$

novos elementos, uma vez que $p(H, n)$ e $q(H, n)$ são primos entre si. De

$$
\frac{q(H, n)}{\operatorname{mdc}(c, q(H, n))} \geq \frac{q(H, n)}{c}>\frac{1}{\epsilon}
$$

segue que a distância entre dois desses novos elementos consecutivos é menor que $\epsilon$. Como $\delta(A) \geq \epsilon$, existe $y \in \mathbb{T}$ tal que $d(H) \cdot y=x$ e $a(H, n) \cdot c \cdot y \in A$.

Lema 4.2.4. Sejam $(H, J) \in(\mathbb{Q} / \mathbb{Z})^{(\omega)} \oplus \mathbb{Q}^{([\omega, \mathfrak{c}[)} \backslash\{(0,0)\}$ e $E \in[\mathfrak{c}]^{\omega}$ satisfazendo as condições

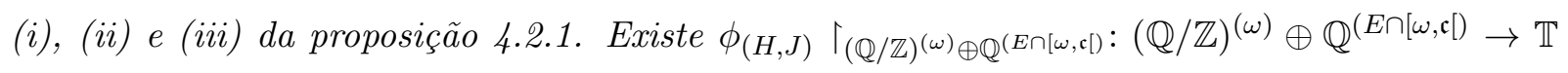
um homomorfismo de grupos com as seguintes propriedades:

(i) $\phi_{(H, J)} \Upsilon_{(\mathbb{Q} / \mathbb{Z})^{(\omega)} \oplus \mathbb{Q}^{(E \cap[\omega, \mathfrak{c}[)}}(H, J) \neq 0+\mathbb{Z}$;

(ii) $\phi_{(H, J)} \Upsilon_{(\mathbb{Q} / \mathbb{Z})^{(\omega)} \oplus \mathbb{Q}^{(E \cap[\omega, \mathfrak{c})}}\left(0, \chi_{\xi}\right)=p_{\xi}-\lim \left\{\phi_{(H, J)} \Upsilon_{(\mathbb{Q} / \mathbb{Z})^{(\omega)} \oplus \mathbb{Q}^{(E \cap[\omega, \mathfrak{c})}}\left(h_{\xi}(n)\right): n \in \omega\right\}$, para cada $\xi \in E \cap L_{0} \cdot{ }^{7}$

Demonstração. Consideremos $\left\{E_{n}: n \in \omega\right\},\left\{b_{n}: n \in \omega\right\},\left\{r_{n}: n \in \omega\right\}$ e $i: \omega \rightarrow E \cap L_{0}$ de acordo com o lema 4.2.2.

Se $H=0$, façamos $\hat{\phi}(H)=0+\mathbb{Z}$. Se $H \neq 0$, seja $\hat{\phi}(H)$ um elemento não nulo de $\mathbb{T}$ tal que $\mathrm{o}(\hat{\phi}(H)) \mid \mathrm{o}(H)$. Em ambos os casos, estendemos $\hat{\phi}$ a um homomorfismo de grupos de $\langle H\rangle \mathrm{em}$

\footnotetext{
${ }^{7}$ Estamos considerando $(\mathbb{Q} / \mathbb{Z})^{(\omega)} \oplus \mathbb{Q}^{(E \cap[\omega, c[)} \subset(\mathbb{Q} / \mathbb{Z})^{(\omega)} \oplus \mathbb{Q}^{([\omega, \mathfrak{c}[)}$.
} 
$\mathbb{T}$. Dados $k \in \omega$ e $s \in \mathbb{N} \backslash\{0\}$, definimos $\Lambda_{k, s}: \omega \rightarrow \mathbb{Q} / \mathbb{Z}$ por

$$
\Lambda_{k, s}(n)=\left\{\begin{array}{ccc}
\frac{1}{s}+\mathbb{Z} & \text { se } & n=k \\
0+\mathbb{Z} & \text { se } & n \neq k
\end{array}\right.
$$

Seja

$$
G_{0}=\left\langle\left\{\Lambda_{k, d(H)}: k \in \operatorname{supp} H\right\}\right\rangle .
$$

Como $\mathbb{T}$ é um grupo divisível e $\langle H\rangle$ é um subgrupo de $G_{0}$, podemos estender $\hat{\phi}$ a um homomorfismo de grupos $\phi \uparrow_{G_{0}}: G_{0} \rightarrow \mathbb{T}$.

Se $J=0$, tomemos $\psi_{0}^{*}(\xi)$ um arco aberto de $\mathbb{T}$ com diâmetro

$$
\delta\left(\psi_{0}^{*}(\xi)\right)=\frac{r_{0}}{d(J)}
$$

e façamos $\psi_{0}(\xi)=d(J) \cdot \psi_{0}^{*}(\xi)$, para todo $\xi \in E_{0} \cap[\omega, \mathfrak{c}[$.

Suponhamos $J \neq 0$. Para cada $\xi \in E_{0} \cap\left[\omega, \mathfrak{c}\left[\right.\right.$, tomemos $y_{\xi} \in \mathbb{R}$ tal que

$$
\sum_{\xi \in \operatorname{supp} J} J(\xi) \cdot y_{\xi}=\frac{1}{2}-x
$$

onde $x \in\left[0,1\left[\right.\right.$ é tal que $x+\mathbb{Z}=\phi \uparrow_{G_{0}}(H)$ e façamos

$$
x_{\xi}=\frac{1}{d(J)} \cdot y_{\xi}+\mathbb{Z}
$$

Temos que

$$
\sum_{\xi \in \operatorname{supp}} a(J, \xi) \cdot x_{\xi}=\left(\frac{1}{2}+\mathbb{Z}\right)-\phi \Upsilon_{G_{0}}(H) .
$$

Seja $\psi_{0}^{*}(\xi)$ o arco aberto de $\mathbb{T}$ centrado em $x_{\xi}$ com diâmetro

$$
\delta\left(\psi_{0}^{*}(\xi)\right)=\frac{r_{0}}{d(J)}
$$

Seja, também, $\psi_{0}(\xi)=d(J) \cdot \psi_{0}^{*}(\xi)$. Claramente, $\delta\left(\psi_{0}(\xi)\right)=r_{0}$. Uma vez que

$$
\frac{1}{2}+\mathbb{Z} \in \phi \uparrow_{G_{0}}(H)+\sum_{\xi \in \operatorname{supp} J} a(J, \xi) \cdot \psi_{0}^{*}(\xi)
$$


$\mathrm{e}$

$\delta\left(\phi\left\lceil G_{0}(H)+\sum_{\xi \in \operatorname{supp} J} a(J, \xi) \cdot \psi_{0}^{*}(\xi)\right)=\delta\left(\sum_{\xi \in \operatorname{supp} J} a(J, \xi) \cdot \psi_{0}^{*}(\xi)\right) \leq \sum_{\xi \in \operatorname{supp} J}|a(J, \xi)| \cdot \delta\left(\psi_{0}^{*}(\xi)\right) \leq \frac{1}{4}\right.$

concluímos que

$$
0+\mathbb{Z} \notin \phi\left\lceil G_{0}(H)+\sum_{\xi \in \operatorname{supp} J} a(J, \xi) \cdot \psi_{0}^{*}(\xi) .\right.
$$

Por fim, se $\xi \in\left(E \backslash E_{0}\right) \cap\left[\omega, \mathfrak{c}\left[\right.\right.$, façamos $\psi_{0}(\xi)=\mathbb{T}$.

Seja $n \in \omega$. Suponhamos definidas $\psi_{n}: E \cap\left[\omega, \mathfrak{c}\left[\rightarrow \mathcal{B}\right.\right.$ e $\psi_{n}^{*}: E_{n} \cap[\omega, \mathfrak{c}[\rightarrow \mathcal{B}$. Suponhamos, também, definido $\phi \Gamma_{G_{n}}: G_{n} \rightarrow \mathbb{T}$ um homomorfismo de grupos, onde

$$
G_{n}=\left\langle\left\{\Lambda_{k, d(H) \cdot \prod_{m<n} d\left(g_{i(m)}\left(b_{m}\right)\right)}: k \in \operatorname{supp} H \cup \bigcup_{m<n} \operatorname{supp} g_{i(m)}\left(b_{m}\right)\right\}\right\rangle .
$$

Vamos definir $\psi_{n+1}: E \cap\left[\omega, \mathfrak{c}\left[\rightarrow \mathcal{B}, \psi_{n+1}^{*}: E_{n+1} \cap\left[\omega, \mathfrak{c}\left[\rightarrow \mathcal{B}\right.\right.\right.\right.$ e estender $\phi \uparrow_{G_{n}}$ a um homomorfismo de grupos $\phi \uparrow_{G_{n+1}}: G_{n+1} \rightarrow \mathbb{T}$, onde

$$
G_{n+1}=\left\langle\left\{\Lambda_{k, d(H) \cdot \prod_{m<n+1} d\left(g_{i(m)}\left(b_{m}\right)\right)}: k \in \operatorname{supp} H \cup \bigcup_{m<n+1} \operatorname{supp} g_{i(m)}\left(b_{m}\right)\right\}\right\rangle
$$

satisfazendo as seguintes condições:

(1) Se $\xi \in\left(E \backslash E_{n+1}\right) \cap\left[\omega, \mathfrak{c}\left[\right.\right.$, então $\psi_{n+1}(\xi)=\mathbb{T}$;

(2) Se $\xi \in\left(E_{n+1} \backslash E_{n}\right) \cap\left[\omega, \mathfrak{c}\left[\right.\right.$, então $\psi_{n+1}^{*}(\xi)$ é tal que:

(a) $d(J) \cdot \prod_{m<n+1} d\left(f_{i(m)}\left(b_{m}\right)\right) \cdot \overline{\psi_{n+1}^{*}(\xi)} \subset \psi_{n}(\xi)$;

(b) $\delta\left(\psi_{n+1}^{*}(\xi)\right)=\frac{r_{n+1}}{d(J) \cdot \prod_{m<n+1} d\left(f_{i(m)}\left(b_{m}\right)\right)}$.

Neste caso, façamos

$$
\psi_{n+1}(\xi)=d(J) \cdot \prod_{m<n+1} d\left(f_{i(m)}\left(b_{m}\right)\right) \cdot \psi_{n+1}^{*}(\xi) .
$$

(3) Se $\xi \in E_{n} \cap\left[\omega, \mathfrak{c}\left[\right.\right.$, então $\psi_{n+1}^{*}(\xi)$ é tal que:

(a) $d\left(f_{i(n)}\left(b_{n}\right)\right) \cdot \overline{\psi_{n+1}^{*}(\xi)} \subset \psi_{n}^{*}(\xi)$;

(b) $\delta\left(\psi_{n+1}^{*}(\xi)\right)=\frac{r_{n+1}}{d(J) \cdot \prod_{m<n+1} d\left(f_{i(m)}\left(b_{m}\right)\right)}$. 
Neste caso, façamos

$$
\psi_{n+1}(\xi)=d(J) \cdot \prod_{m<n+1} d\left(f_{i(m)}\left(b_{m}\right)\right) \cdot \psi_{n+1}^{*}(\xi) .
$$

(4) $\psi_{n}(i(n)) \cap\left(\phi\left\lceil_{G_{n+1}}\left(g_{i(n)}\left(b_{n}\right)\right)+\sum_{\mu \in \operatorname{supp} f_{i(n)}\left(b_{n}\right)} a\left(f_{i(n)}\left(b_{n}\right), \mu\right) \cdot d(J) \cdot \prod_{m<n} d\left(f_{i(m)}\left(b_{m}\right)\right) \cdot\right.\right.$ $\left.\psi_{n+1}^{*}(\mu)\right) \neq \emptyset .^{8}$

Se $\xi \in\left(E \backslash E_{n+1}\right) \cap\left[\omega, \mathfrak{c}\left[\right.\right.$, façamos $\psi_{n+1}(\xi)=\mathbb{T}$. Se $\xi \in\left[\left(E_{n+1} \backslash E_{n}\right) \backslash \operatorname{supp} f_{i(n)}\left(b_{n}\right)\right] \cap[\omega, \mathfrak{c}[$, então $\psi_{n}(\xi)=\mathbb{T}$. Definimos $\psi_{n+1}^{*}(\xi)$ como sendo um elemento de $\mathcal{B}$ com diâmetro

$$
\delta\left(\psi_{n+1}^{*}(\xi)\right)=\frac{r_{n+1}}{d(J) \cdot \prod_{m<n+1} d\left(f_{i(m)}\left(b_{m}\right)\right)}
$$

e façamos

$$
\psi_{n+1}(\xi)=d(J) \cdot \prod_{m<n+1} d\left(f_{i(m)}\left(b_{m}\right)\right) \cdot \psi_{n+1}^{*}(\xi) .
$$

Se $\xi \in\left(E_{n} \backslash \operatorname{supp} f_{i(n)}\left(b_{n}\right)\right) \cap\left[\omega, \mathfrak{c}\left[\right.\right.$, fixemos uma raiz $d\left(f_{i(n)}\left(b_{n}\right)\right)$-ésima do ponto médio de $\psi_{n}^{*}(\xi)$. Definimos $\psi_{n+1}^{*}(\xi)$ como sendo o arco aberto de $\mathbb{T}$ centrado na raiz em questão com diâmetro

$$
\delta\left(\psi_{n+1}^{*}(\xi)\right)=\frac{r_{n+1}}{d(J) \cdot \prod_{m<n+1} d\left(f_{i(m)}\left(b_{m}\right)\right)}
$$

e façamos

$$
\psi_{n+1}(\xi)=d(J) \cdot \prod_{m<n+1} d\left(f_{i(m)}\left(b_{m}\right)\right) \cdot \psi_{n+1}^{*}(\xi) .
$$

Vamos, agora, definir $\psi_{n+1}^{*}(\xi)$ e $\psi_{n+1}(\xi)$ para $\xi \in \operatorname{supp} f_{i(n)}\left(b_{n}\right)$ e estender $\phi\left\lceil_{G_{n}}\right.$ a $\phi\left\lceil G_{n+1}\right.$.

Caso 1: $h_{i(n)}$ é do tipo 1.

Como $\mathbb{T}$ é divisível e $G_{n}$ é um subgrupo de $G_{n+1}$, podemos estender $\phi \Gamma_{G_{n}}$ a um homomorfismo de grupos $\phi\left\lceil_{G_{n+1}}: G_{n+1} \rightarrow \mathbb{T}\right.$. Fixemos $\alpha \in \operatorname{supp} f_{i(n)}\left(b_{n}\right) \backslash E_{n}$. Aplicando o lema 3.2 .3 para $\left[\omega, \mathfrak{c}\left[, d(J) \cdot \prod_{m<n} d\left(f_{i(m)}\left(b_{m}\right)\right), r_{n}, \psi_{n}(i(n))-\phi \uparrow_{G_{n+1}}\left(g_{i(n)}\left(b_{n}\right)\right)\right.\right.$, $E_{n} \cap \operatorname{supp} f_{i(n)}\left(b_{n}\right), f_{i(n)}\left(b_{n}\right), \alpha$ e $\psi_{n}^{*}$, obtemos $\tilde{\psi}: \operatorname{supp} f_{i(n)}\left(b_{n}\right) \rightarrow \mathbb{T}$ satisfazendo as condições (i), (ii), (iii) e (iv) do lema 3.2.3. Façamos $\psi_{n+1}^{*}(\xi)=\tilde{\psi}(\xi)$, para todo $\xi \in \operatorname{supp} f_{i(n)}\left(b_{n}\right)$. As condições $(1),(2),(3)$ e (4) estão satisfeitas.

Caso 2: $h_{i(n)}$ é do tipo 2 .

${ }^{8} \operatorname{Se} f_{i(n)}\left(b_{n}\right)=0$, então $\sum_{\mu \in \operatorname{supp} f_{i(n)}\left(b_{n}\right)} a\left(f_{i(n)}\left(b_{n}\right), \mu\right) \cdot d(J) \cdot \prod_{m<n} d\left(f_{i(m)}\left(b_{m}\right)\right) \cdot \psi_{n+1}^{*}(\mu)=\{0+\mathbb{Z}\}$. 
Como $\mathbb{T}$ é divisível e $G_{n}$ é um subgrupo de $G_{n+1}$, podemos estender $\phi\left\lceil_{G_{n}}\right.$ a um homomorfismo de grupos $\phi\left\lceil G_{n+1}: G_{n+1} \rightarrow \mathbb{T}\right.$. Fixemos $\alpha \in \operatorname{supp} f_{i(n)}\left(b_{n}\right)$ tal que $q\left(f_{i(n)}\left(b_{n}\right), \alpha\right) \cdot r_{n}>d(J) \cdot \prod_{m<n} d\left(f_{i(m)}\left(b_{m}\right)\right)$. Podemos supor que $\alpha \in E_{n}$ pois, do contrário, os argumentos utilizados no caso 1 podem ser repetidos aqui. Aplicando o lema 3.2.4 para $\left[\omega, \mathfrak{c}\left[, d(J) \cdot \prod_{m<n} d\left(f_{i(m)}\left(b_{m}\right)\right), r_{n}, \psi_{n}(i(n))-\phi\left\lceil_{G_{n+1}}\left(g_{i(n)}\left(b_{n}\right)\right), E_{n} \cap \operatorname{supp} f_{i(n)}\left(b_{n}\right)\right.\right.\right.$, $f_{i(n)}\left(b_{n}\right), \alpha$ e $\psi_{n}^{*}$, obtemos $\tilde{\psi}: \operatorname{supp} f_{i(n)}\left(b_{n}\right) \rightarrow \mathbb{T}$ satisfazendo as condições (i), (ii), (iii) e (iv) do lema 3.2.4. Façamos $\psi_{n+1}^{*}(\xi)=\tilde{\psi}(\xi)$, para todo $\xi \in \operatorname{supp} f_{i(n)}\left(b_{n}\right)$. As condições (1), (2), (3) e (4) estão satisfeitas.

Caso 3: $h_{i(n)}$ é do tipo 3 .

Como $\mathbb{T}$ é divisível e $G_{n}$ é um subgrupo de $G_{n+1}$, podemos estender $\phi \Upsilon_{G_{n}}$ a um homomorfismo de grupos $\phi \uparrow_{G_{n+1}}: G_{n+1} \rightarrow \mathbb{T}$. Fixemos $\alpha \in \operatorname{supp} f_{i(n)}\left(b_{n}\right)$ tal que $\left|a\left(f_{i(n)}\left(b_{n}\right), \alpha\right)\right| \cdot r_{n}>4 \cdot d\left(f_{i(n)}\left(b_{n}\right)\right)$. Novamente, podemos supor que $\alpha \in E_{n}$. Aplicando o lema 3.2 .5 para $\left[\omega, \mathfrak{c}\left[, d(J) \cdot \prod_{m<n} d\left(f_{i(m)}\left(b_{m}\right)\right), r_{n}, \psi_{n}(i(n))-\phi \uparrow_{G_{n+1}}\left(g_{i(n)}\left(b_{n}\right)\right)\right.\right.$, $E_{n} \cap \operatorname{supp} f_{i(n)}\left(b_{n}\right), f_{i(n)}\left(b_{n}\right), \alpha$ e $\psi_{n}^{*}$, obtemos $\tilde{\psi}: \operatorname{supp} f_{i(n)}\left(b_{n}\right) \rightarrow \mathbb{T}$ satisfazendo as condições (i), (ii), (iii) e (iv) do lema 3.2.5. Façamos $\psi_{n+1}^{*}(\xi)=\tilde{\psi}(\xi)$, para todo $\xi \in \operatorname{supp} f_{i(n)}\left(b_{n}\right)$. As condições $(1),(2),(3)$ e (4) estão satisfeitas.

Caso 4: $h_{i(n)}$ é do tipo 4 .

Se $\xi \in\left(E_{n+1} \backslash E_{n}\right) \cap \operatorname{supp} f_{i(n)}\left(b_{n}\right)$, definimos $\psi_{n+1}^{*}(\xi)$ como sendo um elemento de $\mathcal{B}$ tal que

$$
\delta\left(\psi_{n+1}^{*}(\xi)\right)=\frac{r_{n+1}}{d(J) \cdot \prod_{m<n+1} d\left(f_{i(m)}\left(b_{m}\right)\right)} .
$$

Se $\xi \in E_{n} \cap \operatorname{supp} f_{i(n)}\left(b_{n}\right)$, fixemos uma raiz $d\left(f_{i(n)}\left(b_{n}\right)\right)$-ésima do ponto médio de $\psi_{n}^{*}(\xi)$. Definimos $\psi_{n+1}^{*}(\xi)$ como sendo o arco aberto de $\mathbb{T}$ centrado na raiz em questão com diâmetro

$$
\delta\left(\psi_{n+1}^{*}(\xi)\right)=\frac{r_{n+1}}{d(J) \cdot \prod_{m<n+1} d\left(f_{i(m)}\left(b_{m}\right)\right)} .
$$

Em ambos os casos, façamos

$$
\psi_{n+1}(\xi)=d(J) \cdot \prod_{m<n+1} d\left(f_{i(m)}\left(b_{m}\right)\right) \cdot \psi_{n+1}^{*}(\xi)
$$

Denotemos por $z_{\xi}$ o centro de $\psi_{n+1}^{*}(\xi)$, para todo $\xi \in \operatorname{supp} f_{i(n)}\left(b_{n}\right)$. 
Seja

$$
\tilde{G}_{n+1}=\left\langle\left\{\Lambda_{k, d(H) \cdot \prod_{m<n} d\left(g_{i(m)}\left(b_{m}\right)\right)}: k \in \operatorname{supp} H \cup \bigcup_{m<n+1} \operatorname{supp} g_{i(m)}\left(b_{m}\right)\right\}\right\rangle .
$$

Como $\mathbb{T}$ é divisível e $G_{n}$ é um subgrupo de $\tilde{G}_{n+1}$, podemos estender $\phi \uparrow_{G_{n}}$ a um homomorfismo de grupos $\phi \uparrow_{\tilde{G}_{n+1}}: \tilde{G}_{n+1} \rightarrow \mathbb{T}$. Fixemos $l \in \operatorname{supp} g_{i(n)}\left(b_{n}\right)$ tal que $q\left(g_{i(n)}\left(b_{n}\right), l\right) \cdot r_{n}>d(H) \cdot \prod_{m<n} d\left(g_{i(m)}\left(b_{m}\right)\right)$. Se $k \in \operatorname{supp} g_{i(n)}\left(b_{n}\right) \backslash\{l\}$, seja $y_{k}$ uma raiz $d\left(g_{i(n)}\left(b_{n}\right)\right)$-ésima de $\phi\left\lceil_{\tilde{G}_{n+1}}\left(\Lambda_{k, d(H) \cdot \prod_{m<n} d\left(g_{i(m)}\left(b_{m}\right)\right)}\right)\right.$.

Aplicando o lema 4.2.3 para $\omega, d(H) \cdot \prod_{m<n} d\left(g_{i(m)}\left(b_{m}\right)\right), r_{n}, \psi_{n}(i(n))-u-v, g_{i(n)}\left(b_{n}\right), l$ e $x=\phi \uparrow_{\tilde{G}_{n+1}}\left(\Lambda_{l, d(H) \cdot \prod_{m<n} d\left(g_{i(m)}\left(b_{m}\right)\right)}\right)$, onde

$$
u=\sum_{k \in \operatorname{supp}} a\left(g_{i(n)}\left(b_{n}\right), k\right) \cdot d(H) \cdot \prod_{m<n} d\left(g_{i(m)}\left(b_{m}\right)\right) \cdot y_{k}
$$

e

$$
v=\sum_{\xi \in \operatorname{supp} f_{i(n)}\left(b_{n}\right)} a\left(f_{i(n)}\left(b_{n}\right), \xi\right) \cdot d(J) \cdot \prod_{m<n} d\left(f_{i(m)}\left(b_{m}\right)\right) \cdot z_{\xi},
$$

obtemos $y_{l} \in \mathbb{T}$ tal que $d\left(g_{i(n)}\left(b_{n}\right)\right) \cdot y_{l}=\phi \uparrow_{\tilde{G}_{n+1}}\left(\Lambda_{l, d(H) \cdot \prod_{m<n} d\left(g_{i(m)}\left(b_{m}\right)\right)}\right) \mathrm{e}$

$$
a\left(g_{i(n)}\left(b_{n}\right), l\right) \cdot d(H) \cdot \prod_{m<n} d\left(g_{i(m)}\left(b_{m}\right)\right) \cdot y_{l}+u+v \in \psi_{n}(i(n))
$$

Estendamos $\phi\left\lceil_{\tilde{G}_{n+1}}\right.$ a um homomorfismo de grupos $\phi\left\lceil_{G_{n+1}}: G_{n+1} \rightarrow \mathbb{T}\right.$ de modo que

$$
\phi\left\lceil G_{n+1}\left(\Lambda_{k, d(H) \cdot \prod_{m<n+1} d\left(g_{i(m)}\left(b_{m}\right)\right)}\right)=y_{k}\right.
$$

para todo $k \in \operatorname{supp} g_{i(n)}\left(b_{n}\right)$. As condições $(1),(2),(3)$ e (4) estão satisfeitas.

Por indução, obtemos aplicações $\psi_{n}: E \cap\left[\omega, \mathfrak{c}\left[\rightarrow \mathcal{B}\right.\right.$ e $\psi_{n}^{*}: E_{n} \cap[\omega, \mathfrak{c}[\rightarrow \mathcal{B}$ para cada $n \in \omega$, satisfazendo as seguintes condições:

- $\overline{\psi_{n+1}(\xi)} \subset \psi_{n}(\xi)$, para todo $\xi \in E \cap[\omega, \mathfrak{c}[$;

- $\delta\left(\psi_{n}(\xi)\right)=r_{n}$, se $\xi \in E_{n} \cap\left[\omega, \mathfrak{c}\left[\right.\right.$ e $\psi_{n}(\xi)=\mathbb{T}$, se $\xi \in\left(E \backslash E_{n}\right) \cap[\omega, \mathfrak{c}[;$

- $\psi_{n}(\xi)=d(J) \cdot \prod_{m<n} d\left(f_{i(m)}\left(b_{m}\right)\right) \cdot \psi_{n}^{*}(\xi)$, se $\xi \in E_{n} \cap[\omega, \mathfrak{c}[$. 
Obtemos, ainda, $\phi\left\lceil_{G}: G \rightarrow \mathbb{T}\right.$ um homomorfismo de grupos, onde $G=\cup_{n \in \omega} G_{n} \subset(\mathbb{Q} / \mathbb{Z})^{(\omega)}$. Usando o fato de $\mathbb{T}$ ser um grupo divisível, estendemos $\phi\left\lceil_{G}\right.$ a um homomorfismo de grupos

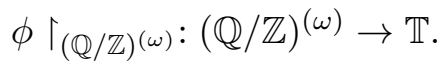

Como $\mathbb{T}$ é um espaço métrico completo e $\left(r_{n}\right)_{n \in \omega}$ é uma seqüência de números reais positivos que converge para 0 , concluímos que se $\xi \in E \cap\left[\omega, \mathfrak{c}\left[\right.\right.$, então $\cap_{n \in \omega} \psi_{n}(\xi)=\cap_{n \in \omega} \overline{\psi_{n}(\xi)}$ é um conjunto unitário. Denotaremos por $\tilde{\phi}\left(\chi_{\xi}\right)$ o único elemento de $\cap_{n \in \omega} \psi_{n}(\xi)$.

Para cada $\xi \in E \cap\left[\omega, \mathfrak{c}\left[\right.\right.$, sejam $N_{\xi}=\min \left\{n \in \omega: \xi \in E_{n}\right\}$ e $n \geq N_{\xi}$. Temos que $\psi_{n}(\xi) \neq \mathbb{T}$ e, portanto, existe um único elemento de $\psi_{n}^{*}(\xi)$ que multiplicado por $d(J) \cdot \prod_{m<n} d\left(f_{i(m)}\left(b_{m}\right)\right)$ é igual a $\tilde{\phi}\left(\chi_{\xi}\right)$. Denotaremos tal elemento por

$$
\tilde{\phi}\left(\frac{1}{d(J) \cdot \prod_{m<n} d\left(f_{i(m)}\left(b_{m}\right)\right)} \cdot \chi_{\xi}\right)
$$

Consideremos

$$
G_{\xi}=\left\{\frac{1}{d(J) \cdot \prod_{m<n} d\left(f_{i(m)}\left(b_{m}\right)\right)} \cdot \chi_{\xi} \in \mathbb{Q}^{(E \cap[\omega, \mathfrak{c}[)}: n \geq N_{\xi}\right\}
$$

e denotemos por $\tilde{G}$ o grupo gerado por $\cup_{\xi \in E \cap[\omega, \mathfrak{c}[} G_{\xi}$. Uma vez que se $\xi \in E \cap\left[\omega, \mathfrak{c}\left[\right.\right.$ e $n>N_{\xi}$, então

$$
\tilde{\phi}\left(\frac{1}{d(J) \cdot \prod_{m<N_{\xi}} d\left(f_{i(m)}\left(b_{m}\right)\right)} \cdot \chi_{\xi}\right)=\prod_{N_{\xi} \leq m<n} d\left(f_{i(m)}\left(b_{m}\right)\right) \cdot \tilde{\phi}\left(\frac{1}{d(J) \cdot \prod_{m<n} d\left(f_{i(m)}\left(b_{m}\right)\right)} \cdot \chi_{\xi}\right)
$$

podemos estender $\tilde{\phi}$ a um homomorfismo de grupos de $\tilde{G}$ em $\mathbb{T}$. Usando o fato de $\mathbb{T}$ ser um

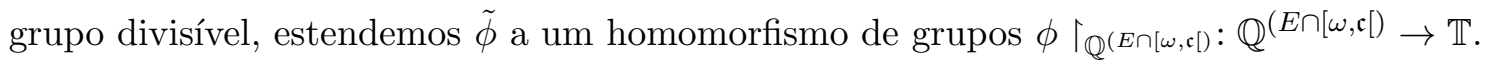

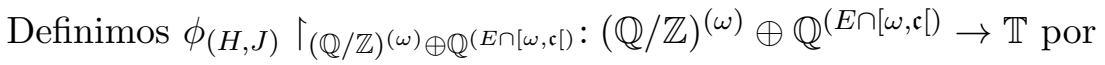

$$
\phi_{(H, J)} \uparrow_{(\mathbb{Q} / \mathbb{Z})^{(\omega)} \oplus \mathbb{Q}^{(E \cap[\omega, \mathfrak{c}[)}}(\tilde{H}, \tilde{J})=\phi \Gamma_{(\mathbb{Q} / \mathbb{Z})^{(\omega)}}(\tilde{H})+\phi \Gamma_{\mathbb{Q}^{(E \cap[\omega, \mathfrak{c})}}(\tilde{J})
$$

para todo $(\tilde{H}, \tilde{J}) \in(\mathbb{Q} / \mathbb{Z})^{(\omega)} \oplus \mathbb{Q}^{(E \cap[\omega, \mathfrak{c}[)}$.

Se $J=0$, então $\phi \Upsilon_{(\mathbb{Q} / \mathbb{Z})^{(\omega)} \oplus \mathbb{Q}^{(E \cap[\omega, c[)}}(H, J)=\phi r_{(\mathbb{Q} / \mathbb{Z})^{(\omega)}}(H) \neq \mathbb{Z}$, uma vez que $(H, J) \neq(0,0)$. Suponhamos, portanto, que $J \neq 0$. Temos que

$$
\phi \Gamma_{\mathbb{Q}^{(E \cap[\omega, \mathfrak{c}[)}}(J) \in \sum_{\xi \in \operatorname{supp} J} a(J, \xi) \cdot \psi_{0}^{*}(\xi) .
$$


Como

$$
0+\mathbb{Z} \notin \phi \uparrow_{(\mathbb{Q} / \mathbb{Z})^{(\omega)}}(H)+\sum_{\xi \in \operatorname{supp} J} a(J, \xi) \cdot \psi_{0}^{*}(\xi)
$$

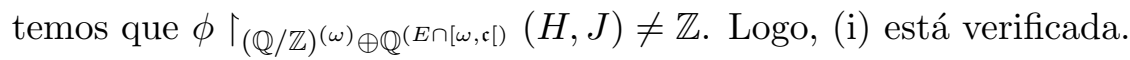

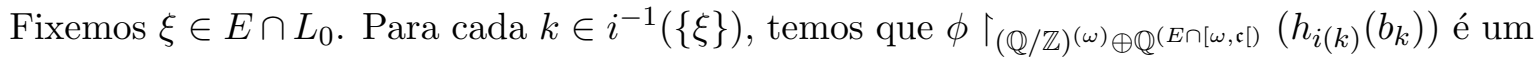
elemento de

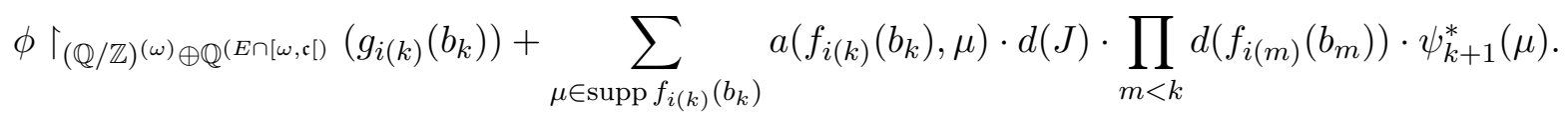

Além disso,

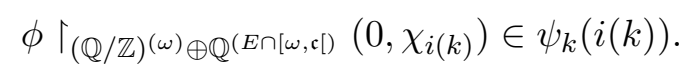

De (4) vem que

$$
\begin{aligned}
\delta\left(\phi \Gamma_{(\mathbb{Q} / \mathbb{Z})^{(\omega)} \oplus \mathbb{Q}^{(E \cap[\omega, \mathfrak{c}[)}}\left(h_{i(k)}\left(b_{k}\right)\right), \phi \Gamma_{(\mathbb{Q} / \mathbb{Z})^{(\omega)} \oplus \mathbb{Q}^{(E \cap[\omega, \mathfrak{c}[)}}\left(0, \chi_{i(k)}\right)\right) & \leq d_{1}+d_{2} \\
& <2 r_{k}
\end{aligned}
$$

onde

$$
d_{1}=\delta\left(\sum_{\mu \in \operatorname{supp} f_{i(k)}\left(b_{k}\right)} a\left(f_{i(k)}\left(b_{k}\right), \mu\right) \cdot d(J) \cdot \prod_{m<k} d\left(f_{i(m)}\left(b_{m}\right)\right) \cdot \psi_{k+1}^{*}(\mu)\right)
$$

e

$$
d_{2}=\delta\left(\psi_{k}(i(k))\right)
$$

Como $r_{k} \rightarrow 0$, concluímos que a seqüência $\left\{\phi \Upsilon_{(\mathbb{Q} / \mathbb{Z})^{(\omega)} \oplus \mathbb{Q}^{(E \cap[\omega, \mathfrak{c}[)}}\left(h_{i(k)}\left(b_{k}\right)\right): k \in i^{-1}(\{\xi\})\right\}$ converge para $\phi \Gamma_{(\mathbb{Q} / \mathbb{Z})^{(\omega)} \oplus \mathbb{Q}^{(E \cap[\omega, \mathfrak{c})}}\left(0, \chi_{\xi}\right)$. Da propriedade $(\mathrm{v})$ do lema 4.2 .2 segue que

$$
\phi \uparrow_{(\mathbb{Q} / \mathbb{Z})^{(\omega)} \oplus \mathbb{Q}^{(E \cap[\omega, \mathfrak{c}[)}}\left(0, \chi_{\xi}\right)=p_{\xi}-\lim \left\{\phi \Upsilon_{(\mathbb{Q} / \mathbb{Z})^{(\omega)} \oplus \mathbb{Q}^{(E \cap[\omega, \mathfrak{c}[)}}\left(h_{\xi}(n)\right): n \in \omega\right\} .
$$

Logo, (ii) está verificada.

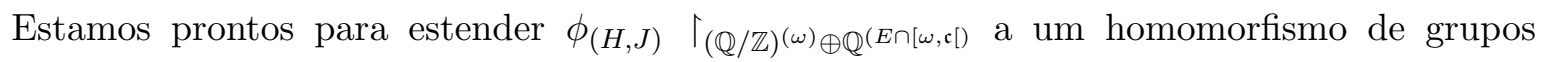
$\phi_{(H, J)}:(\mathbb{Q} / \mathbb{Z})^{(\omega)} \oplus \mathbb{Q}^{([\omega, \mathfrak{c}[)} \rightarrow \mathbb{T}$.

Lema 4.2.5. Para cada $(H, J) \in(\mathbb{Q} / \mathbb{Z})^{(\omega)} \oplus \mathbb{Q}^{([\omega, \mathfrak{c}[)} \backslash\{(0,0)\}$, existe $\phi_{(H, J)}:(\mathbb{Q} / \mathbb{Z})^{(\omega)} \oplus \mathbb{Q}^{([\omega, \mathfrak{c}[)} \rightarrow$ $\mathbb{T}$ um homomorfismo de grupos tal que:

(i) $\phi_{(H, J)}(H, J) \neq 0+\mathbb{Z}$; 
(ii) $\phi_{(H, J)}\left(0, \chi_{\xi}\right)=p_{\xi}-\lim \left\{\phi_{(H, J)}\left(h_{\xi}(n)\right): n \in \omega\right\}$, para cada $\xi \in L_{0}$.

Demonstração. Faremos a construção de $\phi_{(H, J)}$ por indução. De acordo com a proposição 4.2.1, existe $E \in[\mathfrak{c}]^{\omega}$ tal que $\operatorname{supp}(H, J) \subset E,\left|E \cap L_{0}\right|=\omega$ e $\cup_{n \in \omega} \operatorname{supp} h_{\xi}(n) \subset E$, qualquer

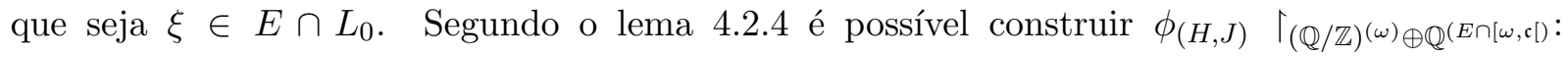
$(\mathbb{Q} / \mathbb{Z})^{(\omega)} \oplus \mathbb{Q}^{(E \cap[\omega, \mathfrak{c}[)} \rightarrow \mathbb{T}$ um homomorfismo de grupos com as seguintes propriedades:

(1) $\phi_{(H, J)} \Upsilon_{(\mathbb{Q} / \mathbb{Z})^{(\omega)} \oplus \mathbb{Q}^{(E \cap[\omega, \mathfrak{c})}}(H, J) \neq 0+\mathbb{Z}$;

(2) $\phi_{(H, J)} \Upsilon_{(\mathbb{Q} / \mathbb{Z})^{(\omega)} \oplus \mathbb{Q}^{(E \cap[\omega, \mathfrak{c})}}\left(0, \chi_{\xi}\right)=p_{\xi}-\lim \left\{\phi_{(H, J)} \Upsilon_{(\mathbb{Q} / \mathbb{Z})^{(\omega)} \oplus \mathbb{Q}^{(E \cap[\omega, \mathfrak{c})}}\left(h_{\xi}(n)\right): n \in \omega\right\}$, para cada $\xi \in E \cap L_{0}$.

Consideremos $\left\{\alpha_{\xi}: \xi<\mathfrak{c}\right\}$ uma indexação estritamente crescente de $[\omega, \mathfrak{c}[\backslash E$ e fixemos

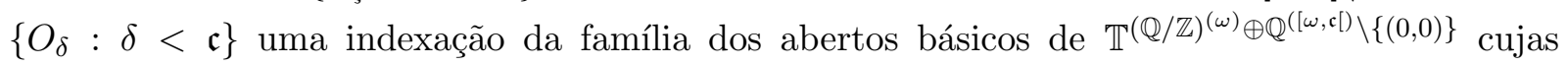
projeções em cada coordenada são arcos abertos de $\mathbb{T}$ com extremos racionais ou o próprio $\mathbb{T}$. Suponhamos que $O_{0}=\mathbb{T}^{(\mathbb{Q} / \mathbb{Z})^{(\omega)} \oplus \mathbb{Q}^{([\omega, \mathfrak{c}[)} \backslash\{(0,0)\}}$. Dado $\delta<\mathfrak{c}$, escreveremos

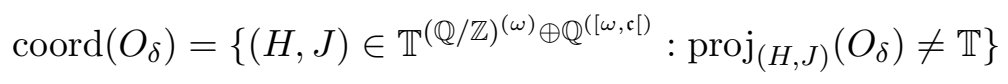

onde

$$
\operatorname{proj}_{(H, J)}(x)=x(H, J)
$$

para todo $x \in \mathbb{T}^{(\mathbb{Q} / \mathbb{Z})^{(\omega)} \oplus \mathbb{Q}^{([\omega, \mathfrak{c}[)} \backslash\{(0,0)\}}$. Para cada $\beta \in \mathfrak{c} \backslash\{0\}$, fixemos $\left\{L_{\beta, \delta}: \delta<\mathfrak{c}\right\}$ uma partição de $L_{\beta}$ tal que $\left|L_{\beta, \delta}\right|=\mathfrak{c}$, para todo $\delta<\mathfrak{c}$.

- Se $\alpha_{0} \in L_{0}$, definamos

$$
\tilde{\phi}_{(H, J)}\left(0, \chi_{\alpha_{0}}\right)=p_{\alpha_{0}}-\lim \left\{\phi_{(H, J)} \Upsilon_{(\mathbb{Q} / \mathbb{Z})^{(\omega)} \oplus \mathbb{Q}^{(E \cap[\omega, \mathfrak{c})}}\left(h_{\alpha_{0}}(n)\right): n \in \omega\right\}
$$

e façamos

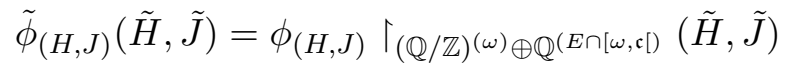

para todo $(\tilde{H}, \tilde{J}) \in(\mathbb{Q} / \mathbb{Z})^{(\omega)} \oplus \mathbb{Q}^{(E \cap[\omega, \mathfrak{c}[)} .^{9}$

- Se existem $\beta \in \mathfrak{c} \backslash\{0\}$ e $\delta<\mathfrak{c}$ tais que $\alpha_{0} \in L_{\beta, \delta}$, definamos $\tilde{\phi}_{(H, J)}\left(0, \chi_{\alpha_{0}}\right)$ de modo que

$$
\tilde{\phi}_{(H, J)}\left(x^{\alpha_{0}}, \chi_{\alpha_{0}}\right) \in \operatorname{proj}_{(H, J)}\left(O_{\delta}\right)
$$

\footnotetext{
${ }^{9}$ Estamos considerando $\mathbb{Q}^{(E \cap[\omega, \mathfrak{c}[)} \subset \mathbb{Q}^{\left(\left(E \cap\left[\omega, \mathfrak{c}[) \cup\left\{\alpha_{0}\right\}\right)\right.\right.} \subset \mathbb{Q}^{([\omega, \mathfrak{c}[)}$.
} 
e façamos

$$
\tilde{\phi}_{(H, J)}(\tilde{H}, \tilde{J})=\phi_{(H, J)} \uparrow_{(\mathbb{Q} / \mathbb{Z})^{(\omega)} \oplus \mathbb{Q}^{(E \cap[\omega, \mathfrak{c})}}(\tilde{H}, \tilde{J})
$$

para todo $(\tilde{H}, \tilde{J}) \in(\mathbb{Q} / \mathbb{Z})^{(\omega)} \oplus \mathbb{Q}^{(E \cap[\omega, \mathfrak{c}[)}$.

- Se $\alpha_{0} \notin \cup_{\beta<\mathfrak{c}} L_{\beta}$, definamos $\tilde{\phi}_{(H, J)}\left(0, \chi_{\alpha_{0}}\right)$ arbitrariamente e façamos

$$
\tilde{\phi}_{(H, J)}(\tilde{H}, \tilde{J})=\phi_{(H, J)} \Upsilon_{(\mathbb{Q} / \mathbb{Z})^{(\omega)} \oplus \mathbb{Q}^{(E \cap[\omega, \mathfrak{c})}}(\tilde{H}, \tilde{J})
$$

para todo $(\tilde{H}, \tilde{J}) \in(\mathbb{Q} / \mathbb{Z})^{(\omega)} \oplus \mathbb{Q}^{(E \cap[\omega, \mathfrak{c}[)}$.

Seja $G_{0}$ o subgrupo de $\mathbb{Q}^{\left(\left(E \cap\left[\omega, \mathfrak{c}[) \cup\left\{\alpha_{0}\right\}\right)\right.\right.}$ gerado por $\mathbb{Q}^{(E \cap[\omega, \mathfrak{c}[)} \cup\left\{\chi_{\alpha_{0}}\right\}$. Podemos estender $\tilde{\phi}_{(H, J)}$ a um homomorfismo de grupos de $(\mathbb{Q} / \mathbb{Z})^{(\omega)} \oplus G_{0}$ em $\mathbb{T}$. Uma vez que $\mathbb{T}$ é divisível, estendemos $\tilde{\phi}_{(H, J)}$ a um homomorfismo de grupos $\phi_{(H, J)} \uparrow_{(\mathbb{Q} / \mathbb{Z})^{(\omega)} \oplus \mathbb{Q}\left(\left(E \cap\left[\omega, \mathfrak{c}[) \cup\left\{\alpha_{0}\right\}\right)\right.\right.}:(\mathbb{Q} / \mathbb{Z})^{(\omega)} \oplus$ $\mathbb{Q}^{\left(\left(E \cap\left[\omega, \mathfrak{c}[) \cup\left\{\alpha_{0}\right\}\right)\right.\right.} \rightarrow \mathbb{T}$.

Repetindo indutivamente esta construção, obteremos $\phi_{(H, J)}:(\mathbb{Q} / \mathbb{Z})^{(\omega)} \oplus \mathbb{Q}^{([\omega, \mathfrak{c}[)} \rightarrow \mathbb{T}$ um homomorfismo de grupos satisfazendo (i) e (ii).

\subsection{Explicitando a topologia}

A próxima proposição mostra que o produto diagonal da família $\left\{\phi_{(H, J)}:(H, J) \in\right.$ $\left.(\mathbb{Q} / \mathbb{Z})^{(\omega)} \oplus \mathbb{Q}^{([\omega, \mathfrak{c}[)} \backslash\{(0,0)\}\right\}$ é um monomorfismo de grupos.

Proposição 4.3.1. Suponhamos que para cada $(H, J) \in(\mathbb{Q} / \mathbb{Z})^{(\omega)} \oplus \mathbb{Q}^{([\omega, \mathfrak{c}[)} \backslash\{(0,0)\}$ exista um homomorfismo de grupos $\phi_{(H, J)}:(\mathbb{Q} / \mathbb{Z})^{(\omega)} \oplus \mathbb{Q}^{([\omega, \mathfrak{c}[)} \rightarrow \mathbb{T}$ tal que $\phi_{(H, J)}(H, J) \neq 0+\mathbb{Z}$. A aplicação

$$
\begin{array}{cccc}
\Phi: \quad(\mathbb{Q} / \mathbb{Z})^{(\omega)} \oplus \mathbb{Q}^{([\omega, \mathfrak{c}[)} & \rightarrow & \mathbb{T}^{(\mathbb{Q} / \mathbb{Z})^{(\omega)} \oplus \mathbb{Q}^{([\omega, \mathfrak{c}[)} \backslash\{(0,0)\}} \\
(\tilde{H}, \tilde{J}) & \mapsto & \Phi(\tilde{H}, \tilde{J})
\end{array}
$$

dada por

$$
\Phi(\tilde{H}, \tilde{J})(H, J)=\phi_{(H, J)}(\tilde{H}, \tilde{J}), \operatorname{para} \operatorname{cada}(H, J) \in(\mathbb{Q} / \mathbb{Z})^{(\omega)} \oplus \mathbb{Q}^{([\omega, \mathfrak{c}[)} \backslash\{(0,0)\}
$$

é um monomorfismo de grupos.

Demonstração. Análoga à da proposição 2.3.1. 
Teorema 4.3.2. Se $G$ é um grupo abeliano quase livre de torção tal que $|G|=\mathfrak{c}$, então $G$ admite uma topologia que o torna um grupo topológico (regular) enumeravelmente compacto sem seqüências não triviais convergentes.

Demonstração. Seja

$$
\sigma=\left\{\varphi^{-1} \circ \Phi^{-1}(U \cap \Phi[\varphi[G]]): U \text { é aberto em } \mathbb{T}^{(\mathbb{Q} / \mathbb{Z})^{(\omega)} \oplus \mathbb{Q}^{([\omega, \mathfrak{c}[)} \backslash\{(0,0)\}}\right\}
$$

Temos que $\sigma$ é uma topologia em $G$ que o torna um grupo topológico (regular).

Consideremos $h: \omega \rightarrow G$. Se $h$ é trivial, nada temos a fazer. Se não, $h$ possui duas subseqüências constantes e distintas ou existe $j: \omega \rightarrow \omega$ estritamente crescente tal que $h \circ j$ é injetora. No primeiro caso, temos que $\{h(n) \in G: n \in \omega\}$ tem pelo menos dois pontos de acumulação distintos e, portanto, $h$ não é convergente. Suponhamos que o segundo caso ocorra.

As aplicações

$$
\begin{aligned}
h_{0}: \omega & \rightarrow G \\
n & \mapsto(h \circ j)(2 n)
\end{aligned}
$$

e

$$
\begin{aligned}
h_{1}: \omega & \rightarrow G \\
n & \mapsto(h \circ j)(2 n+1)
\end{aligned}
$$

são subseqüências distintas de $h$. De acordo com a proposição 4.1.2, existem $j_{0}, j_{1}: \omega \rightarrow \omega$ estritamente crescentes tais que $\varphi \circ h_{0} \circ j_{0}, \varphi \circ h_{1} \circ j_{1} \in \mathcal{H}$, uma vez que $h \circ j$ é injetora. Logo, existem $\xi_{0}, \xi_{1} \in L_{0}$ distintos tais que $\varphi \circ h_{0} \circ j_{0}=h_{\xi_{0}}$ e $\varphi \circ h_{1} \circ j_{1}=h_{\xi_{1}}$.

Dado $i \in\{0,1\}$, temos que

$$
\phi_{(H, J)}\left(0, \chi_{\xi_{i}}\right)=p_{\xi_{i}}-\lim \left\{\phi_{(H, J)}\left(h_{\xi_{i}}(n)\right): n \in \omega\right\}
$$

para todo $(H, J) \in(\mathbb{Q} / \mathbb{Z})^{(\omega)} \oplus \mathbb{Q}^{([\omega, \mathfrak{c}[)} \backslash\{(0,0)\}$ e, portanto,

$$
\Phi\left(0, \chi_{\xi_{i}}\right)=p_{\xi_{i}}-\lim \left\{\Phi\left(h_{\xi_{i}}(n)\right): n \in \omega\right\}
$$

Logo,

$$
\varphi^{-1}\left(0, \chi_{\xi_{i}}\right)=p_{\xi_{i}}-\lim \left\{\left(h_{i} \circ j_{i}\right)(n): n \in \omega\right\}
$$

Assim, $\varphi^{-1}\left(0, \chi_{\xi_{0}}\right)$ e $\varphi^{-1}\left(0, \chi_{\xi_{1}}\right)$ são pontos de acumulação distintos de $\{h(n): n \in \omega\}$. 


\subsection{Topologias independentes}

Definição 4.4.1. [35] Sejam $\tau_{1}$ e $\tau_{2}$ duas topologias $T_{1}$ e não discretas em um conjunto $X$. Dizemos que $\tau_{1}$ e $\tau_{2}$ são independentes se $\tau_{1} \cap \tau_{2}$ coincide com a topologia cofinita em $X$.

Observamos que se $(G, \tau)$ é um grupo topológico abeliano, então $G[n]$ é um subconjunto fechado de $G$ para todo $n \in \mathbb{N}$. Portanto, se $G$ é um grupo de não torção, uma condição necessária para que $G$ admita uma topologia independente de $\tau$ é ser quase livre de torção.

A prova da próxima proposição baseia-se na demonstração de um caso particular do teorema de Čech-Pospísil, o qual afirma que se $X$ é um espaço compacto de Hausdorff tal que $\chi(x, X) \geq \omega$ para todo $x \in X$, então $|X| \geq \mathfrak{c}$.

Proposição 4.4.2. Se $X$ é um espaço topológico regular, enumeravelmente compacto e sem seqüências não triviais convergentes, então $|X| \geq \mathfrak{c}$.

Demonstração. Para cada $n \in \omega$, vamos construir uma família $\left\{K_{f}: f \in 2^{n}\right\}$ de subconjuntos fechados infinitos de $X$ tal que:

(1) Se $f \in 2^{n}$ e $m<n$, então $K_{f} \subset K_{f \uparrow_{m}}$;

(2) Se $f, g \in 2^{n}$ e $f \neq g$, então $K_{f} \cap K_{g}=\emptyset$.

Se $n=0$, então $2^{n}=\{\emptyset\}$. Façamos $K_{\emptyset}=X$. Fixemos $n \in \omega$ e suponhamos que $\left\{K_{f}: f \in 2^{n}\right\}$ seja uma família de subconjuntos fechados infinitos de $X$ satisfazendo as condições (1) e (2). Para cada $f \in 2^{n}$, consideremos $f_{0}, f_{1} \in 2^{n+1}$ tais que $f_{0} \uparrow_{n}=f, f_{1} \uparrow_{n}=f, f_{0}(n)=0$ e $f_{1}(n)=1$. Como $K_{f}$ é um subconjunto fechado infinito de $X$, existem $x, y \in K_{f}$ pontos de acumulação distintos de $K_{f}$ em $X .{ }^{10}$ Da regularidade de $X$ segue que existem $U$ e $V$ subconjuntos abertos de $X$ tais que $x \in U, y \in V$ e $\bar{U} \cap \bar{V}=\emptyset$. Uma vez que $x$ e $y$ são pontos de acumulação de $K_{f}$ em $X$, temos que $U \cap K_{f}$ e $V \cap K_{f}$ são subconjuntos infinitos de $X$. Como $K_{f}$ é fechado em $X$, temos que $\overline{U \cap K_{f}} \subset K_{f}$ e $\overline{V \cap K_{f}} \subset K_{f}$. Definimos $K_{f_{1}}=\overline{U \cap K_{f}}$ e $K_{f_{2}}=\overline{V \cap K_{f}}$. Temos

\footnotetext{
${ }^{10}$ Seja $F$ um subconjunto fechado infinito de $X$. Do fato de $X$ ser enumeravelmente compacto decorre que existe $x \in F$ um ponto de acumulação de $F$ em $X$. Suponhamos que, para cada vizinhança aberta $U$ de $x$ em $X$, o conjunto $F \backslash U$ seja finito. Seja $\left\{x_{n}: n \in \omega\right\}$ uma enumeração injetora de $F$. Temos que $\left\{x_{n}: n \in \omega\right\} \backslash U$ é um conjunto finito, para toda vizinhança aberta $U$ de $x \operatorname{em~} X$. Isto implica que a seqüência não trivial $\left\{x_{n}: n \in \omega\right\}$ de $X$ converge para $x$, o que é absurdo. Logo, existe uma vizinhança aberta $U$ de $x$ em $X$ tal que $F \backslash U$ é um subconjunto infinito de $X$. Da compacidade enumerável de $X$ segue que existe $y$ um ponto de acumulação de $F \backslash U$ (e, portanto, de $F$ ) em $X$. Como $F \backslash U$ é um subconjunto fechado de $X$, temos que $y \in F \backslash U$ e, portanto, $x \neq y$.
} 
que $\left\{K_{f}: f \in 2^{n+1}\right\}=\left\{K_{f_{0}}: f \in 2^{n}\right\} \cup\left\{K_{f_{1}}: f \in 2^{n}\right\}$ é uma família de subconjuntos fechados infinitos de $X$ que satisfaz as condições (1) e (2).

Para cada $f \in 2^{\omega}$, seja $K_{f}=\cap_{n \in \omega} K_{f \uparrow_{n}}$. De (1) segue que $K_{f} \neq \emptyset$, uma vez que $X$ é enumeravelmente compacto. De (2) decorre que se $f, g \in 2^{\omega}$ e $f \neq g$, então $K_{f} \cap K_{g}=\emptyset$. Logo, $|F| \geq \mathfrak{c}$.

Corolário 4.4.3. Seja $X$ um espaço topológico regular, enumeravelmente compacto e sem seqüências não triviais convergentes. Se F é um subconjunto fechado e infinito de $X$, então $|F| \geq \mathfrak{c}$.

Demonstração. Basta observar que se $F$ é um subconjunto fechado e infinito de $X$, então $F$ é um espaço topológico regular, enumeravelmente compacto e sem seqüências não triviais convergentes. Da proposição 4.4.2 segue que $|F| \geq \mathfrak{c}$.

Teorema 4.4.4. Se $G$ é um grupo abeliano quase livre de torção e existe uma topologia $\tau$ em $G$ tal que $|G|=|\tau|=\mathfrak{c}$, então existe uma topologia $\sigma$ em $G$ com as seguintes propriedades:

(i) $(G, \sigma)$ é um grupo topológico;

(ii) $\tau$ e $\sigma$ são independentes.

Demonstração. Seja $\sigma$ a topologia em $G$ dada pelo teorema 4.3.2. De acordo com o corolário 4.4.3, a fim de mostrar que $\tau$ e $\sigma$ são independentes, é suficiente verificar que $G \backslash F_{\beta} \notin \sigma$, para todo $\beta \in] 0, \mathfrak{c}[$.

Fixemos $\beta \in \mathfrak{c} \backslash\{0\}$. Se mostrarmos que $\Phi\left[\varphi\left(F_{\beta}\right)\right] \cap O_{\delta} \neq \emptyset$ para todo $\delta<\mathfrak{c}$, teremos que $\Phi\left[\varphi\left(F_{\beta}\right)\right]$ será denso em $\mathbb{T}^{(\mathbb{Q} / \mathbb{Z})^{(\omega)} \oplus \mathbb{Q}}{ }^{([\omega, \mathfrak{c}[)} \backslash\{(0,0)\}$, uma vez que $\left\{O_{\delta}: \delta<\mathfrak{c}\right\}$ constitui uma base de abertos para $\mathbb{T}^{(\mathbb{Q} / \mathbb{Z})^{(\omega)} \oplus \mathbb{Q}^{([\omega, c])} \backslash\{(0,0)\}}$. Logo, $F_{\beta}$ não será um subconjunto fechado de $(G, \sigma)$, uma vez que $F_{\beta} \neq G$.

Temos que $\varphi\left[F_{\beta}\right]=\left(\varphi_{2} \circ \varphi_{1}\right)\left[F_{\beta}\right] \supset \varphi_{2}\left[T_{\beta}\right]=\left\{\left(x^{\beta}, \chi_{\xi}\right): \xi \in L_{\beta}\right\}$. Portanto, $\Phi\left[\varphi\left[F_{\beta}\right]\right] \supset$ $\Phi\left[\left\{\left(x^{\beta}, \chi_{\xi}\right): \xi \in L_{\beta}\right\}\right]$. Claramente, $\Phi\left[\left\{\left(x^{\beta}, \chi_{\xi}\right): \xi \in L_{\beta}\right\}\right] \cap O_{0} \neq \emptyset$. Seja $\delta<\mathfrak{c}$ e suponhamos escolhidos $\left\{\xi_{\gamma}: \gamma<\delta\right\} \subset L_{\beta}$ tais que $\Phi\left(x^{\beta}, \chi_{\xi_{\gamma}}\right) \in O_{\gamma}$. Seja $\operatorname{coord}\left(O_{\delta}\right)=\left\{\left(H_{0}, J_{0}\right), \ldots,\left(H_{k}, J_{k}\right)\right\}$. Temos que $L_{\beta, \delta} \backslash\left(\left\{\xi_{\gamma}: \gamma<\delta\right\} \cup \bigcup_{i=0}^{k} E_{\left(H_{i}, J_{i}\right)}\right) \neq \emptyset$, onde $E_{\left(H_{i}, J_{i}\right)}$ é obtido através da proposição 4.2.1 aplicada a $\left(H_{i}, J_{i}\right)$. Tomemos $\xi_{\delta} \in L_{\beta, \delta} \backslash\left(\left\{\xi_{\gamma}: \gamma<\delta\right\} \cup \bigcup_{i=0}^{k} E_{\left(H_{i}, J_{i}\right)}\right) \neq \emptyset$. Do lema 4.2.5 segue que $\phi_{\left(H_{i}, J_{i}\right)}\left(x^{\beta}, \chi_{\xi_{\delta}}\right) \in \operatorname{proj}_{\left(H_{i}, J_{i}\right)}\left(O_{\delta}\right)$, para todo $i<k+1$. Portanto, $\Phi\left(x^{\beta}, \chi_{\xi_{\delta}}\right) \in O_{\delta}$. 
Corolário 4.4.5. Se $G$ é um grupo abeliano quase livre de torção tal que $|G|=\mathfrak{c}$ e existe uma topologia $\tau$ em $G$ tal que $w(G, \tau)=\omega$, então existe uma topologia $\sigma$ em $G$ com as seguintes propriedades:

(i) $(G, \sigma)$ é um grupo topológico;

(ii) $\tau$ e $\sigma$ são independentes.

Demonstração. Basta observar que $|\tau| \leq 2^{w(G, \tau)}=\mathfrak{c}$ e que, como $(G, \tau)$ é $T_{1}$, então $|\tau| \geq|G|=\mathfrak{c}$. Logo, $|\tau|=\mathfrak{c}$, o que nos permite aplicar o teorema 4.4.4. 

CAPÍTULO 5

\section{Caracterização algébrica dos grupos abelianos de não torção que têm cardinalidade $\mathfrak{c}$ e que admitem uma topologia de grupo enumeravelmente \\ compacta}

Neste capítulo, assumiremos a existência de $\mathfrak{c}$ ultrafiltros seletivos dois a dois incomparáveis (segundo a ordem de Rudin-Keisler) para caracterizar algebricamente os grupos abelianos de não torção que têm cardinalidade $\mathfrak{c}$ e que admitem uma topologia de grupo enumeravelmente compacta sem seqüências não triviais convergentes.

Seja $G$ um grupo abeliano de não torção tal que $|G|=\mathfrak{c}$. Sabemos que se $G$ admite uma topologia de grupo enumeravelmente compacta, então $|G / T(G)|=\mathfrak{c}$ e, para quaisquer $n, d \in \mathbb{N} \backslash\{0\}$ tais que $d \mid n$, o conjunto $d G[n]$ é finito ou tem cardinalidade $\mathfrak{c}$. Nosso objetivo é mostrar que estas condições algébricas também são suficientes para garantir a existência de uma topologia de grupo enumeravelmente compacta em $G$.

A principal diferença com relação ao capítulo anterior é a necessidade de atribuir pontos de acumulação de ordem finita a algumas seqüências em $G$. Começaremos selecionando candidatos adequados e imergindo $G$ em uma soma direta conveniente de cópias de $\mathbb{Q} / \mathbb{Z}$ e $\mathbb{Q}$. A idéia de "reduzir" a quantidade de seqüências para as quais serão atribuídos pontos de acumulação préfixados também se faz presente neste capítulo e, a fim de escolhê-las adequadamente, recorremos à noção de subconjuntos $n$-rounds de um grupo fixado, onde $n$ é um número natural não nulo. 
Encerraremos este capítulo mostrando que se um grupo abeliano de não torção e cardinalidade $\mathfrak{c}$ admite uma topologia de grupo enumeravelmente compacta sem seqüências não triviais convergentes, então o mesmo admite uma topologia de grupo enumeravelmente compacta sem seqüências não triviais convergentes com peso $2^{\mathfrak{c}}$.

\subsection{Selecionando candidatos a pontos de acumulação}

Seja $G$ um grupo abeliano tal que:

- $|G|=\mathfrak{c}$;

- $|G / T(G)|=\mathfrak{c}$;

- $\forall n, d \in \mathbb{N} \backslash\{0\}(d|n \rightarrow| d G[n] \mid<\omega$ ou $|d G[n]|=\mathfrak{c})$.

Consideremos $\left\{P_{0}, P_{1}\right\}$ uma partição de $\mathfrak{c}$ tal que $\left|P_{0}\right|=\left|P_{1}\right|=\mathfrak{c}, \omega \cup\{\omega\} \subset P_{1}$ e $\{\omega+n: n \geq 1\} \subset P_{0}$.

Afirmação 5.1.1. Existem $L_{1} \in\left[P_{1}\right]^{\mathfrak{c}}$ e $\tilde{\varphi}: G \rightarrow(\mathbb{Q} / \mathbb{Z})^{\left(P_{0}\right)} \oplus \mathbb{Q}^{\left(P_{1}\right)}$ um monomorfismo de grupos tais que $\omega \cup\{\omega\} \subset L_{1}$ e $\left\{\left(0, \chi_{\xi}\right) \in(\mathbb{Q} / \mathbb{Z})^{\left(P_{0}\right)} \oplus \mathbb{Q}^{\left(P_{1}\right)}: \xi \in L_{1}\right\} \subset \tilde{\varphi}[G]$.

Demonstração. Seja $\varphi_{1}: G \rightarrow(\mathbb{Q} / \mathbb{Z})^{\left(P_{0}\right)} \oplus \mathbb{Q}^{\left(P_{1}\right)}$ um monomorfismo de grupos. ${ }^{1}$ Por hipótese, $|G / T(G)|=\mathfrak{c}$. Logo, existe $W$ um subconjunto de $G$ tal que:

- $w \notin T(G)$, para todo $w \in W$;

- $w_{1}-w_{2} \notin T(G)$, quaisquer que sejam $w_{1}, w_{2} \in W$ com $w_{1} \neq w_{2}$.

Tomemos $y_{0} \in W$ e definamos $Y_{0}=\left\{y_{0}\right\}$. Seja $\alpha<\mathfrak{c}$ um ordinal. Para cada $\beta<\alpha$, suponhamos definido $Y_{\beta}=\left\{y_{\gamma}: \gamma<\beta\right\}$ um subconjunto independente de $G$ tal que:

- $Y_{\beta} \subset W$

- Se $\gamma<\beta<\alpha$, então $Y_{\gamma} \subset Y_{\beta}$.

\footnotetext{
${ }^{1}$ Como $G$ é um grupo abeliano, segue que $G$ é isomorfo a um subgrupo de um grupo divisível. Além disso, todo grupo divisível é isomorfo a uma soma direta de cópias de $\mathbb{Q}$ e de $\mathbb{Z}_{p}(\infty) \subset \mathbb{Q} / \mathbb{Z}$, para $p$ número primo. Uma vez que $|G|=\mathfrak{c}$, bastam $\mathfrak{c}$ cópias de $\mathbb{Q}$ e de $\mathbb{Q} / \mathbb{Z}$.
} 
Se $\alpha$ for um ordinal limite, façamos $Y_{\alpha}=\cup_{\beta<\alpha} Y_{\beta}$. Suponhamos, portanto, que $\alpha$ seja um ordinal sucessor - digamos, $\alpha=\beta+1$. Afirmamos que existe $y_{\alpha} \in W$ tal que $\left\langle y_{\alpha}\right\rangle \cap\left\langle Y_{\beta}\right\rangle=\{0\}$. Com efeito, se não existisse um tal elemento, então para cada $w \in W$ existiria $m_{w} \in \mathbb{Z} \backslash\{0\}$ de modo que $m_{w} \cdot w \in\left\langle Y_{\beta}\right\rangle$. Todavia, $\left|\left\langle Y_{\beta}\right\rangle\right| \leq \max \{\omega,|\beta|\}<\mathfrak{c}$. Como $|W|=\mathfrak{c}$, existiriam $y \in\left\langle Y_{\beta}\right\rangle$ e $\tilde{W} \subset W$ tais que:

- $|\tilde{W}|=\mathfrak{c}$;

- $m_{w} \cdot w=y$, para todo $w \in \tilde{W}$.

Uma vez que $|\mathbb{Z} \backslash\{0\}|=\omega$, seria possível tomar $\tilde{\tilde{W}} \subset \tilde{W}$ e $m \in \mathbb{Z} \backslash\{0\}$ tais que:

- $|\tilde{\tilde{W}}|=\mathfrak{c}$;

- $m \cdot w=y$, para todo $w \in \tilde{\tilde{W}}$.

Todavia, isto contradiz o fato de que a diferença de dois elementos distintos quaisquer de $W$ não pertence a $T(G)$. Portanto, existe $y_{\alpha} \in W$ tal que $\left\langle y_{\alpha}\right\rangle \cap\left\langle Y_{\beta}\right\rangle=\{0\}$. Façamos $Y_{\alpha}=Y_{\beta} \cup\left\{y_{\alpha}\right\}$. Temos que $Y_{\alpha}$ é um subconjunto independente de $G$ tal que $Y_{\alpha} \subset W$ e $Y_{\beta} \subset Y_{\alpha}$, qualquer que seja $\beta<\alpha$. Seja $Y=\cup_{\alpha<\mathfrak{c}} Y_{\alpha}$.

Temos que $Y \subset W$ é um subconjunto independente de $G$ de cardinalidade $\mathfrak{c}$. Consideremos $\left\{z_{\xi}: \xi<\mathfrak{c}\right\}$ uma indexação de $Y$ e escrevamos

$$
\varphi_{1}\left(z_{\xi}\right)=\left(a_{\xi}, b_{\xi}\right) \in(\mathbb{Q} / \mathbb{Z})^{\left(P_{0}\right)} \oplus \mathbb{Q}^{\left(P_{1}\right)}
$$

qualquer que seja $\xi<\mathfrak{c}$. Para cada $n \in \omega$, seja

$$
A_{n}=\left\{\xi<\mathfrak{c}: n \cdot a_{\xi}=0\right\}
$$

Como $|Y|=\mathfrak{c}$, existe $n \in \omega$ tal que $\left|A_{n}\right|=\mathfrak{c}$. Fixemos um tal $n$ e olhemos para o conjunto $\left\{n \cdot z_{\xi}: \xi \in A_{n}\right\}$. Observamos que o mesmo tem cardinalidade $\mathfrak{c}$, uma vez que $\left|A_{n}\right|=\mathfrak{c}$ e $Y \subset W$ é um subconjunto independente de $G$. Além disso, $\varphi_{1}\left(n \cdot z_{\xi}\right)=\left(0, n \cdot b_{\xi}\right)$, para todo $\xi \in A_{n}$.

Tomemos $\left\{c_{\zeta}: \zeta \in P_{1}\right\}$ uma base do $\mathbb{Q}$-espaço vetorial $\mathbb{Q}^{\left(P_{1}\right)}$ que contém $\left\{n \cdot b_{\xi}: \xi \in A_{n}\right\}$ e tal que $\left\{c_{\zeta}: \zeta \in \omega \cup\{\omega\}\right\} \subset\left\{n \cdot b_{\xi}: \xi \in A_{n}\right\}$. Seja $\varphi_{2}:(\mathbb{Q} / \mathbb{Z})^{\left(P_{0}\right)} \oplus \mathbb{Q}^{\left(P_{1}\right)} \rightarrow(\mathbb{Q} / \mathbb{Z})^{\left(P_{0}\right)} \oplus \mathbb{Q}^{\left(P_{1}\right)}$ o isomorfismo de grupos dado por $\varphi_{2}(a, 0)=(a, 0)$, qualquer que seja $(a, 0) \in(\mathbb{Q} / \mathbb{Z})^{\left(P_{0}\right)} \oplus \mathbb{Q}^{\left(P_{1}\right)} \mathrm{e}$ $\varphi_{2}\left(0, \sum_{\zeta \in F} \alpha_{\zeta} \cdot c_{\zeta}\right)=\left(0, \sum_{\zeta \in F} \alpha_{\zeta} \cdot \chi_{\zeta}\right)$, qualquer que seja $F \in\left[P_{1}\right]^{<\omega} \operatorname{com}\left\{\alpha_{\zeta}: \zeta \in F\right\} \subset \mathbb{Q} \backslash\{0\}$. 
Tomemos $L_{1} \in\left[P_{1}\right]^{\mathfrak{c}}$ tal que $\left|P_{1} \backslash L_{1}\right|=\mathfrak{c}$ e $\left\{n \cdot b_{\xi}: \xi \in A_{n}\right\}=\left\{c_{\zeta}: \zeta \in L_{1}\right\}$. Temos que $\omega \cup\{\omega\} \subset L_{1}$ e $\left\{\left(0, \chi_{\xi}\right): \xi \in L_{1}\right\} \subset \tilde{\varphi}[G]$, onde $\tilde{\varphi}=\varphi_{2} \circ \varphi_{1}$.

Denotemos por $D$ o conjunto dos números naturais $n>1$ tais que $G$ contém uma cópia de $\mathbb{Z}_{n}^{(\mathfrak{c})}$ - e, portanto, um subconjunto independente de cardinalidade $\mathfrak{c}$ constituído de elementos de ordem $n$.

Afirmação 5.1.2. Para cada $n \in D$, existe $\left\{\left(x_{\xi}^{n}, 0\right) \in(\mathbb{Q} / \mathbb{Z})^{\left(P_{0}\right)} \oplus \mathbb{Q}^{\left(P_{1}\right)}: \xi<\mathfrak{c}\right\} \subset \tilde{\varphi}[G]$ com as seguintes propriedades:

(i) $\mathrm{o}\left(x_{\xi}^{n}\right)=n$, para todo $\xi<\mathfrak{c}$;

(ii) $\operatorname{supp} x_{\xi}^{n} \cap \operatorname{supp} x_{\mu}^{n}=\emptyset$, quaisquer que sejam $\xi, \mu<\mathfrak{c}$ com $\xi \neq \mu$.

Demonstração. Sejam $n \in D$ e $\left\{\left(y_{\xi}^{n}, 0\right) \in(\mathbb{Q} / \mathbb{Z})^{\left(P_{0}\right)} \oplus \mathbb{Q}^{\left(P_{1}\right)}: \xi<\mathfrak{c}\right\} \subset \tilde{\varphi}[G]$ independente com $\mathrm{o}\left(y_{\xi}^{n}\right)=n$, para todo $\xi<\mathfrak{c}$.

Caso 1: $\mathfrak{c}$ é regular.

Aplicando o lema do $\Delta$-sistema para $\left\{\operatorname{supp} y_{\xi}^{n}: \xi<\mathfrak{c}\right\}$, obtemos $I_{n} \in[\mathfrak{c}]^{\mathfrak{c}}$ e $R_{n} \in\left[P_{0}\right]^{<\omega}$ tais que $\left\{\operatorname{supp} y_{\xi}^{n}: \xi \in I_{n}\right\}$ constitui um $\Delta$-sistema de raiz $R_{n}$. Tomemos $J_{n} \in\left[I_{n}\right]^{\mathfrak{c}}$ tal que se $\xi, \mu \in J_{n}$, então $y_{\xi}^{n}(\zeta)=y_{\mu}^{n}(\zeta)$ para todo $\zeta \in R_{n} \cdot{ }^{2}$ Seja $\left\{J_{n, 0}, J_{n, 1}\right\}$ uma partição de $J_{n}$ tal que $\left|J_{n, 0}\right|=\left|J_{n, 1}\right|=\mathfrak{c}$. Fixemos $f: \mathfrak{c} \rightarrow J_{n, 0}$ e $g: \mathfrak{c} \rightarrow J_{n, 1}$ bijeções e definamos $x_{\xi}^{n}=y_{f(\xi)}^{n}-y_{g(\xi)}^{n}$, para todo $\xi<\mathfrak{c}$.

Dado $\xi<\mathfrak{c}$, temos que o $\left(x_{\xi}^{n}\right)=n$, uma vez que $n \cdot x_{\xi}^{n}=n \cdot\left(y_{f(\xi)}^{n}-y_{g(\xi)}^{n}\right)=$ $n \cdot y_{f(\xi)}^{n}-n \cdot y_{g(\xi)}^{n}=0-0=0$ e se $m$ é um número natural não nulo menor que $n$, então $m \cdot x_{\xi}^{n}=m \cdot y_{f(\xi)}^{n}-m \cdot y_{g(\xi)}^{n} \neq 0$, uma vez que o $\left(y_{f(\xi)}^{n}\right)=\mathrm{o}\left(y_{g(\xi)}^{n}\right)=n$ e $\left\{y_{\xi}^{n}: \xi<\mathfrak{c}\right\}$ é um subconjunto independente de $G$.

Por fim, sejam $\xi, \mu<\mathfrak{c}$ tais que $\xi \neq \mu$. Se $\zeta \in \operatorname{supp} x_{\xi}^{n} \cap \operatorname{supp} x_{\mu}^{n}$, então uma das seguintes possibilidades ocorre:

- $\zeta \in \operatorname{supp} y_{f(\xi)}^{n} \cap \operatorname{supp} y_{f(\mu)}^{n}$;

- $\zeta \in \operatorname{supp} y_{f(\xi)}^{n} \cap \operatorname{supp} y_{g(\mu)}^{n}$;

- $\zeta \in \operatorname{supp} y_{g(\xi)}^{n} \cap \operatorname{supp} y_{f(\mu)}^{n}$;

- $\zeta \in \operatorname{supp} y_{g(\xi)}^{n} \cap \operatorname{supp} y_{g(\mu)}^{n}$.

\footnotetext{
${ }^{2}$ Um tal $J_{n}$ existe, uma vez que $R_{n}$ é finito, $\mathbb{Q} / \mathbb{Z}$ é enumerável e $\left|I_{n}\right|=\mathfrak{c}$.
} 
Logo, $\zeta \in R_{n}$ e, portanto, $\zeta \in \operatorname{supp} y_{f(\xi)}^{n} \cap \operatorname{supp} y_{f(\mu)}^{n} \cap \operatorname{supp} y_{g(\xi)}^{n} \cap \operatorname{supp} y_{g(\mu)}^{n}$. Como $f(\xi), g(\xi), f(\mu), g(\mu) \in J_{n}$, temos que $y_{f(\xi)}^{n}(\zeta)=y_{f(\mu)}^{n}(\zeta)=y_{g(\xi)}^{n}(\zeta)=y_{g(\mu)}^{n}(\zeta)$ e, portanto, $x_{\xi}^{n}(\zeta)=x_{\mu}^{n}(\zeta)=0$ - o que é absurdo, pois tomamos $\zeta \in \operatorname{supp} x_{\xi}^{n} \cap \operatorname{supp} x_{\mu}^{n}$. Portanto, $\operatorname{supp} x_{\xi}^{n} \cap \operatorname{supp} x_{\mu}^{n}=\emptyset$, quaisquer que sejam $\xi, \mu<\mathfrak{c} \operatorname{com} \xi \neq \mu$.

Caso 2: $\mathfrak{c}$ não é regular.

Neste caso, $\operatorname{cf}(\mathfrak{c})<\mathfrak{c}$ e $\mathfrak{c}$ é um cardinal limite.

Seja $\left\{I_{\alpha}: \alpha<\operatorname{cf}(\mathfrak{c})\right\}$ uma família de subconjuntos de $\mathfrak{c}$ dois a dois disjuntos tal que $\mathfrak{c}=\cup_{\alpha<\operatorname{cf}(\mathfrak{c})} I_{\alpha}$ e $\left|I_{\alpha}\right|=\kappa_{\alpha}$, para cada $\alpha<\operatorname{cf}(\mathfrak{c})$, onde:

- $\left\{\kappa_{\alpha}: \alpha<\operatorname{cf}(\mathfrak{c})\right\}$ é uma seqüência estritamente crescente e cofinal em $\mathfrak{c}$;

- $\kappa_{\alpha}$ é um cardinal regular, para todo $\alpha<\mathfrak{c}$;

- $\kappa_{\alpha} \geq \max \left\{\omega,|\alpha|, \sup _{\beta<\alpha} \kappa_{\beta}\right\}^{+3}$

Para cada $\alpha<\operatorname{cf}(\mathfrak{c})$ podemos repetir a construção feita no caso 1 e obter $\left\{\left(x_{\xi}^{n}, 0\right) \in\right.$ $\left.(\mathbb{Q} / \mathbb{Z})^{\left(P_{0}\right)} \oplus \mathbb{Q}^{\left(P_{1}\right)}: \xi \in I_{\alpha}\right\} \subset \tilde{\varphi}[G]$ tal que o $\left(x_{\xi}^{n}\right)=n$ para todo $\xi \in I_{\alpha}$ e $\operatorname{supp} x_{\xi}^{n} \cap \operatorname{supp} x_{\mu}^{n}=$ $\emptyset$, quaisquer que sejam $\xi, \mu \in I_{\alpha} \operatorname{com} \xi \neq \mu$.

Sejam $J_{0}=I_{0}$ e $\alpha<\operatorname{cf}(\mathfrak{c})$ um ordinal. Suponhamos que para cada $\beta<\alpha$, exista $J_{\beta} \subset I_{\beta}$ tal que $\left|J_{\beta}\right|=\left|I_{\beta}\right|=\kappa_{\beta}$ e $\operatorname{supp} x_{\xi}^{n} \cap \operatorname{supp} x_{\mu}^{n}=\emptyset$, quaisquer que sejam $\xi, \mu \in \cup_{\beta<\alpha} J_{\beta}$ com $\xi \neq \mu$. Seja $X_{\alpha}=\cup_{\xi \in \cup_{\beta<\alpha} J_{\beta}} \operatorname{supp} x_{\xi}^{n}$. Temos que $\left|X_{\alpha}\right|<\kappa_{\alpha}$ e, portanto, existe $J_{\alpha} \subset I_{\alpha}$ tal que $\left|J_{\alpha}\right|=\left|I_{\alpha}\right|$ e $\operatorname{supp} x_{\xi}^{n} \cap X_{\alpha}=\emptyset$, para todo $\xi \in J_{\alpha}{ }^{4}$

Definimos $J=\cup_{\alpha<\operatorname{cf}(\mathfrak{c})} J_{\alpha}$. Temos que $|J|=\mathfrak{c} \operatorname{e} \operatorname{supp} x_{\xi}^{n} \cap \operatorname{supp} x_{\mu}^{n}=\emptyset$, quaisquer que sejam $\xi, \mu \in J \operatorname{com} \xi \neq \mu$.

\footnotetext{
${ }^{3}$ Seja $\left\{\lambda_{\alpha}: \alpha<\operatorname{cf}(\mathfrak{c})\right\}$ uma seqüência estritamente crescente e cofinal em $\mathfrak{c}$. Definamos $\kappa_{0}=\max \left\{\omega,\left|\lambda_{0}\right|^{+}\right\}^{+}$. Temos que $\kappa_{0}>\omega$ e $\kappa_{0}<\mathfrak{c}$, uma vez que $\mathfrak{c}$ é um cardinal limite e $\lambda_{0}<\mathfrak{c}$. Seja $\alpha<\operatorname{cf}(\mathfrak{c})$ e suponhamos que $\kappa_{\beta}$ esteja definido para todo $\beta<\alpha$, de modo que:

$-\max \left\{|\beta|,\left|\lambda_{\beta}\right|^{+}\right\}<\kappa_{\beta}<\mathfrak{c}$

- $\kappa_{\beta}$ é um cardinal regular;

- Se $\gamma<\beta<\alpha$, então $\kappa_{\gamma}<\kappa_{\beta}$.

Definimos $\kappa_{\alpha}=\max \left\{|\alpha|,\left|\lambda_{\alpha}\right|^{+}, \sup _{\beta<\alpha} \kappa_{\beta}\right\}^{+}$. Temos que $\max \left\{|\alpha|,\left|\lambda_{\alpha}\right|^{+}\right\}<\kappa_{\alpha}$ e como $\alpha<\operatorname{cf}(\mathfrak{c})$, temos que $\sup _{\beta<\alpha} \kappa_{\beta}<\mathfrak{c}$. Logo, $\kappa_{\alpha}<\mathfrak{c}$, uma vez que $\mathfrak{c}$ é um cardinal limite. Do fato de $\kappa_{\alpha}$ ser um cardinal sucessor decorre que $\kappa_{\alpha}$ é um cardinal regular. Por fim, observamos que se $\beta<\alpha$, então $\kappa_{\beta}<\kappa_{\alpha}$. Por indução em cf(c), construímos $\left\{\kappa_{\alpha}: \alpha<\operatorname{cf}(\mathfrak{c})\right\}$ satisfazendo as condições listadas acima. Façamos $I_{0}=\kappa_{0}$ e, para cada $\alpha<\operatorname{cf}(\mathfrak{c})$, tomamos $I_{\alpha}=\kappa_{\alpha} \backslash \cup_{\beta<\alpha} \kappa_{\beta}$. Temos que $\mathfrak{c}=\dot{U}_{\alpha<\operatorname{cf}(\mathfrak{c})} I_{\alpha}$ e $\left|I_{\alpha}\right|=\kappa_{\alpha}$, para todo $\alpha<\operatorname{cf}(\mathfrak{c})$.

${ }^{4}$ Isto ocorre uma vez que $\operatorname{supp} x_{\xi}^{n} \cap \operatorname{supp} x_{\mu}^{n}=\emptyset$, quaisquer que sejam $\xi, \mu \in I_{\alpha} \operatorname{com} \xi \neq \mu$.
} 
Afirmação 5.1.3. Para cada $n \in D$, existe $L_{n} \in\left[P_{0}\right]^{\mathfrak{c}}$ tal que $L_{m} \cap L_{n}=\emptyset$, quaisquer que sejam $m, n \in D$ com $m \neq n$. Além disso, existe $\left\{\left(x_{\xi}, 0\right) \in(\mathbb{Q} / \mathbb{Z})^{\left(P_{0}\right)} \oplus \mathbb{Q}^{\left(P_{1}\right)}: \xi \in \cup_{n \in D} L_{n}\right\} \subset \tilde{\varphi}[G]$ com as seguintes propriedades:

(i) $S e \xi \in L_{n}$, então o $\left(x_{\xi}\right)=n$;

(ii) Se $\xi, \mu \in \cup_{n \in D} L_{n}$ e $\xi \neq \mu$, então $\operatorname{supp} x_{\xi} \cap \operatorname{supp} x_{\mu}=\emptyset$;

(iii) $\xi \in \operatorname{supp} x_{\xi}$, para todo $\xi \in \cup_{n \in D} L_{n}$.

Demonstração. Para cada $n \in D$, seja $\left\{x_{\xi}^{n}: \xi<\mathfrak{c}\right\}$ onde $\left\{\left(x_{\xi}^{n}, 0\right) \in(\mathbb{Q} / \mathbb{Z})^{\left(P_{0}\right)} \oplus \mathbb{Q}^{\left(P_{1}\right)}: \xi<\right.$ c $\} \subset \tilde{\varphi}[G]$ satisfaz as condições (i) e (ii) da afirmação 5.1.2. Consideremos $\left\{X_{\zeta}: \zeta<\mathfrak{c}\right\}$ uma indexação de $\left\{\left\{x_{\xi}^{n}: \xi<\mathfrak{c}\right\}: n \in D\right\}$ de modo que

$$
\left|\left\{\zeta<\mathfrak{c}: X_{\zeta}=\left\{x_{\xi}^{n}: \xi<\mathfrak{c}\right\}\right\}\right|=\mathfrak{c}
$$

para todo $n \in D$.

Fixemos $x \in X_{0}$ e definamos $\xi_{0}=\min \operatorname{supp} x$. Denotaremos $x$ por $x_{\xi_{0}}$. Seja $\alpha<\mathfrak{c}$ um ordinal. Para cada $\beta<\alpha$, suponhamos definidos $\xi_{\beta} \in P_{0}$ e $x_{\xi_{\beta}} \in X_{\beta}$ com as seguintes propriedades:

- $\xi_{\beta}=\min \operatorname{supp} x_{\xi_{\beta}}$;

- Se $\gamma<\beta<\alpha$, então $\operatorname{supp} x_{\xi_{\gamma}} \cap \operatorname{supp} x_{\xi_{\beta}}=\emptyset$.

Temos que $\left|\cup_{\beta<\alpha} \operatorname{supp} x_{\xi_{\beta}}\right| \leq \max \{|\alpha|, \omega\}<\mathfrak{c}$. Uma vez que $\left|X_{\alpha}\right|=\mathfrak{c} \operatorname{e} \operatorname{supp} x \cap \operatorname{supp} y=\emptyset$ para quaisquer $x$ e $y$ elementos distintos de $X_{\alpha}$, existe $z \in X_{\alpha}$ tal que $\operatorname{supp} z \cap\left(\cup_{\beta<\alpha} \operatorname{supp} x_{\xi_{\beta}}\right)=$ $\emptyset$. Definamos $\xi_{\alpha}=\min \operatorname{supp} z$ e escrevamos $x_{\xi_{\alpha}}=z$. Por indução, obtemos $\xi_{\alpha} \in P_{0}$ e $x_{\xi_{\alpha}} \in X_{\alpha}$, para todo $\alpha<\mathfrak{c}$.

Se $n \in D$, definimos

$$
L_{n}=\left\{\xi_{\alpha} \in P_{0}: X_{\alpha}=\left\{x_{\xi}^{n}: \xi<\mathfrak{c}\right\}\right\} \backslash\{\omega+m: m \geq 1\}
$$

Note que $\left|L_{n}\right|=\mathfrak{c}$, uma vez que $\left|\left\{\alpha<\mathfrak{c}: X_{\alpha}=\left\{x_{\xi}^{n}: \xi<\mathfrak{c}\right\}\right\}\right|=\mathfrak{c}$ e $\xi_{\alpha} \neq \xi_{\beta}$ se $\alpha$ e $\beta$ são elementos distintos de c. Além disso, $L_{m} \cap L_{n}=\emptyset$, se $m, n \in D$ e $m \neq n$. Se $\xi \in L_{n}$, então $\mathrm{o}\left(x_{\xi}\right)=n$ e se $\xi, \mu \in \cup_{n \in D} L_{n}$ e $\xi \neq \mu$, então $\operatorname{supp} x_{\xi} \cap \operatorname{supp} x_{\mu}=\emptyset$. Por fim, se $\xi \in \cup_{n \in D} L_{n}$, então $\xi \in \operatorname{supp} x_{\xi}$. 
Proposição 5.1.4. Existe $\varphi: G \rightarrow(\mathbb{Q} / \mathbb{Z})^{\left(P_{0} \times \omega\right)} \oplus \mathbb{Q}^{\left(P_{1}\right)}$ um monomorfismo de grupos tal que $\left\{\left(0, \chi_{\xi}\right) \in(\mathbb{Q} / \mathbb{Z})^{\left(P_{0} \times \omega\right)} \oplus \mathbb{Q}^{\left(P_{1}\right)}: \xi \in L_{1}\right\} \subset \varphi[G] e\left\{\left(y_{\xi}, 0\right) \in(\mathbb{Q} / \mathbb{Z})^{\left(P_{0} \times \omega\right)} \oplus \mathbb{Q}^{\left(P_{1}\right)}: \xi \in\right.$ $\left.\cup_{n \in D} L_{n}\right\} \subset \varphi[G]$, onde:

(i) $S e \xi \in L_{n}$, então o $\left(y_{\xi}\right)=n$;

(ii) $S e \xi \in \cup_{n \in D} L_{n}$, então $\operatorname{supp} y_{\xi} \subset\{\xi\} \times \omega$.

Demonstração. Seja $\left\{\left(x_{\xi}, 0\right) \in(\mathbb{Q} / \mathbb{Z})^{\left(P_{0}\right)} \oplus \mathbb{Q}^{\left(P_{1}\right)}: \xi \in \cup_{n \in D} L_{n}\right\} \subset \tilde{\varphi}[G]$ satisfazendo as condições (i), (ii) e (iii) da afirmação 5.1.3. Consideremos a aplicação $\hat{\varphi}:(\mathbb{Q} / \mathbb{Z})^{\left(P_{0}\right)} \oplus \mathbb{Q}^{\left(P_{1}\right)} \rightarrow$ $(\mathbb{Q} / \mathbb{Z})^{\left(P_{0} \times \omega\right)} \oplus \mathbb{Q}^{\left(P_{1}\right)}$ definida da seguinte maneira:

- Seja $\xi \in L_{n}$, para algum $n \in D$. Denotemos $\operatorname{supp} x_{\xi}$ por $\left\{\alpha_{0}, \alpha_{1}, \ldots, \alpha_{m}\right\}$, onde $\alpha_{0}<\alpha_{1}<$ $\ldots<\alpha_{m}$. A $\alpha_{i}$-ésima parcela $\mathbb{Q} / \mathbb{Z}$ da soma direta $(\mathbb{Q} / \mathbb{Z})^{\left(P_{0}\right)}$ será levada identicamente na $(\xi, i)$-ésima parcela da soma direta $(\mathbb{Q} / \mathbb{Z})^{\left(P_{0} \times \omega\right)}$.

- Se $\mu \in P_{0} \backslash \cup_{\xi \in \cup_{n \in D} L_{n}} \operatorname{supp} x_{\xi}$, então a $\mu$-ésima parcela $\mathbb{Q} / \mathbb{Z}$ da soma direta $(\mathbb{Q} / \mathbb{Z})^{\left(P_{0}\right)}$ será levada identicamente na $(\mu, 0)$-ésima parcela da soma direta $(\mathbb{Q} / \mathbb{Z})^{\left(P_{0} \times \omega\right)}$.

- $\hat{\varphi}(0, b)=(0, b)$, para todo $b \in \mathbb{Q}^{\left(P_{1}\right)}$.

A aplicação $\hat{\varphi}$ é um monomorfismo de grupos. Sejam $\varphi=\hat{\varphi} \circ \tilde{\varphi}$ e $\left(y_{\xi}, 0\right)=\hat{\varphi}\left(x_{\xi}, 0\right)$, para todo $\xi \in \cup_{n \in D} L_{n}$. Temos que $\varphi$ é um monomorfismo de grupos tal que

$$
\left\{\left(0, \chi_{\xi}\right) \in(\mathbb{Q} / \mathbb{Z})^{\left(P_{0} \times \omega\right)} \oplus \mathbb{Q}^{\left(P_{1}\right)}: \xi \in L_{1}\right\} \subset \varphi[G]
$$

e

$$
\left\{\left(y_{\xi}, 0\right) \in(\mathbb{Q} / \mathbb{Z})^{\left(P_{0} \times \omega\right)} \oplus \mathbb{Q}^{\left(P_{1}\right)}: \xi \in \cup_{n \in D} L_{n}\right\} \subset \varphi[G]
$$

Além disso, se $\xi \in L_{n}$, então o $\left(y_{\xi}\right)=\mathrm{o}\left(\hat{\varphi}\left(x_{\xi}, 0\right)\right)=n$, uma vez que $\hat{\varphi}$ é um monomorfismo de grupos. Por fim, supp $y_{\xi} \subset\{\xi\} \times \omega$, qualquer que seja $\xi \in \cup_{n \in D} L_{n}$.

\subsection{Uma escolha adequada de seqüências}

Se $(H, J) \in(\mathbb{Q} / \mathbb{Z})^{\left(P_{0} \times \omega\right)} \oplus \mathbb{Q}^{\left(P_{1}\right)}$, então

$$
H(\xi, n)=\frac{p(H,(\xi, n))}{q(H,(\xi, n))}+\mathbb{Z}
$$


onde $p(H,(\xi, n)), q(H,(\xi, n)) \in \mathbb{Z}, q(H,(\xi, n))>0, \operatorname{mdc}(p(H,(\xi, n)), q(H,(\xi, n)))=1$ e $0 \leq p(H,(\xi, n)) \leq q(H,(\xi, n))-1$ para cada $(\xi, n) \in \operatorname{supp} H$. Além disso,

$$
J(\mu)=\frac{p(J, \mu)}{q(J, \mu)}
$$

onde $p(J, \mu), q(J, \mu) \in \mathbb{Z}, q(J, \mu)>0$ e $\operatorname{mdc}(p(J, \mu), q(J, \mu))=1$, para cada $\mu \in \operatorname{supp} J$. Sejam

$$
\begin{gathered}
|q(H)|=\max \{q(H,(\xi, n)):(\xi, n) \in \operatorname{supp} H\}, \\
|q(J)|=\max \{q(J, \mu): \mu \in \operatorname{supp} J\}
\end{gathered}
$$

e

$$
|p(J)|=\max \{|p(J, \mu)|: \mu \in \operatorname{supp} J\} .
$$

Consideremos

$$
d(H)=\operatorname{mmc}\{q(H,(\xi, n)):(\xi, n) \in \operatorname{supp} H\}
$$

e

$$
d(J)=\operatorname{mmc}\{q(J, \mu): \mu \in \operatorname{supp} J\} .^{5}
$$

Para cada $(\xi, n) \in \operatorname{supp} H$, seja

$$
a(H,(\xi, n))=p(H,(\xi, n)) \cdot \frac{d(H)}{q(H,(\xi, n))}
$$

e, para cada $\mu \in \operatorname{supp} J$, seja

$$
a(J, \mu)=p(J, \mu) \cdot \frac{d(J)}{q(J, \mu)} .
$$

Sejam, ainda,

$$
|a(J)|=\max \{|a(J, \mu)|: \mu \in \operatorname{supp} J\}
$$

$\mathrm{e}$

$$
\|a(H, J)\|=\sum_{(\xi, n) \in \operatorname{supp} H}|a(H,(\xi, n))|+\sum_{\mu \in \operatorname{supp} J}|a(J, \mu)| .
$$

Observamos que

$$
\operatorname{supp}(H, J)=\operatorname{supp} H \cup \operatorname{supp} J
$$

\footnotetext{
${ }^{5}$ Se $H=0$, então $d(H)=1$. Analogamente, se $J=0$, então $d(J)=1$.
} 
Se $H \in(\mathbb{Q} / \mathbb{Z})^{\left(P_{0} \times \omega\right)}$ e $X \subset P_{0} \times \omega$, então $H \uparrow_{X} \in(\mathbb{Q} / \mathbb{Z})^{\left(P_{0} \times \omega\right)}$ é dada por

$$
H \uparrow_{X}(\xi, n)=\left\{\begin{array}{lll}
H(\xi, n) & \text { se } & (\xi, n) \in X \\
0+\mathbb{Z} & \text { se } & (\xi, n) \notin X
\end{array}\right.
$$

para todo $(\xi, n) \in P_{0} \times \omega$. Por fim, se $X \subset P_{0} \times \omega$, escreveremos

$$
\pi_{1}[X]=\left\{\xi \in P_{0}: \exists n \in \omega \text { tal que }(\xi, n) \in X\right\}
$$

e

$$
\pi_{2}[X]=\left\{n \in \omega: \exists \xi \in P_{0} \text { tal que }(\xi, n) \in X\right\} .
$$

Definição 5.2.1. [17] Sejam $S \subset G$ infinito e $n>1$ um número natural. Dizemos que $S$ é $n$-round se:

- $S \subset G[n]$;

- A restrição do homomorfismo de grupos

$$
\begin{aligned}
\varphi_{d}: G & \rightarrow G \\
x & \mapsto d x
\end{aligned}
$$

a $S$ é finita-a-um, qualquer que seja d divisor próprio de $n .^{6}$

As duas proposições seguintes encontram-se em [17]. Como suas demonstrações são curtas, optamos por reproduzi-las aqui.

Proposição 5.2.2. [17] Sejam $n>1$ um número natural e $S$ um subconjunto infinito de $G[n]$. Existem $T \subset G$ infinito e $z \in G$ tais que $T+z \subset S$ e $T$ éd-round, para algum divisor d de $n$.

Demonstração. Seja $S \subset G[n]$ infinito. Se $S$ é $n$-round, tomamos $T=S$ e $z=0$. Se não, existe $d$ um divisor próprio de $n$ tal que a restrição de $\varphi_{d}$ a $S$ não é finita-a-um. Tomemos $d$ minimal com respeito a esta propriedade. Existem $g \in G$ e $S^{\prime} \subset S$ infinito tais que $\varphi_{d}(x)=g$, para todo $x \in S^{\prime}$. Fixemos $z \in S^{\prime}$ e façamos $T=S^{\prime}-z$. Temos que $T \subset G[d]$. Além disso, se $r$ é um divisor próprio de $d$, então $r$ é, em particular, um divisor próprio de $n$ e $r<d$. Logo, a restrição de $\varphi_{r}$ a $S$ é finita-a-um. Daí segue que a restrição de $\varphi_{r}$ a $T$ é finita-a-um e, portanto, $T$ é $d$-round. Por fim, $T+z=\left(S^{\prime}-z\right)+z \subset S^{\prime} \subset S$.

\footnotetext{
${ }^{6}$ Isto é, $\varphi_{d}^{-1}(\{x\})$ é um subconjunto finito de $G$, qualquer que seja $x \in G$.
} 
Proposição 5.2.3. [17] Seja $n>1$ um número natural. Se existe $S \subset G$ tal que $S$ é $n$-round, então $n \in D$.

Demonstração. Observamos que $|S| \geq \omega$ e que $S \subset G[n]$. Como $|G[n]|<\omega$ ou $|G[n]|=\mathfrak{c}$, temos que $|G[n]|=\mathfrak{c}$. Para cada divisor próprio $d$ de $n$, temos que $d S$ é um subconjunto infinito de $d G[n]$ e como $|d G[n]|<\omega$ ou $|d G[n]|=\mathfrak{c}$, concluímos que $|d G[n]|=\mathfrak{c}$. Vamos mostrar que $\mathbb{Z}_{n}^{(\mathfrak{c})}$ é isomorfo a um subgrupo de $G$ por indução no número de divisores de $n$.

Se $n$ é um número primo, então $G[n]$ é isomorfo à soma direta de $\mathfrak{c}$ cópias de $\mathbb{Z}_{n}^{(\mathfrak{c})}$ e, portanto, $n \in D$. Suponhamos, agora, que $n=m p$, onde $m>1$ é um número natural e $p$ é um número primo. Temos que $p S$ é um subconjunto $m$-round de $G{ }^{7}$ Como o número de divisores de $m$ é estritamente menor que o número de divisores de $n$ temos, por hipótese de indução, que $m \in D$. Isto significa que $\mathbb{Z}_{m}^{(\mathfrak{c})}$ é isomorfo a um subgrupo de $G$. Podemos escrever $m=m_{0} p^{k}$, onde $\operatorname{mdc}\left(m_{0}, p\right)=1$. Observamos que $G[n]=G\left[m_{0} p^{k+1}\right] \cong G\left[m_{0}\right] \oplus G\left[p^{k+1}\right]$. Do teorema de 1.1.3 decorre que $G\left[p^{k+1}\right] \cong \oplus_{i=1}^{k+1} \mathbb{Z}_{p^{i}}^{\left(\theta_{i}\right)}$, onde $\theta_{1}, \ldots, \theta_{k+1}$ são cardinais menores ou iguais a c. Uma vez que $|m G[n]|=\mathfrak{c}$ e

$$
m G[n] \cong m_{0} p^{k}\left(G\left[m_{0}\right] \oplus G\left[p^{k+1}\right]\right) \cong m_{0} p^{k} G\left[p^{k+1}\right] \cong m_{0} p^{k} \oplus \mathbb{Z}_{p^{i}}^{\left(\theta_{i}\right)}
$$

concluímos que $\theta_{k+1}=\mathfrak{c}$. Logo, $\mathbb{Z}_{p^{k+1}}^{(\mathfrak{c})}$ é isomorfo a um subgrupo de $G$, o que significa que $p^{k+1} \in D$. Portanto, se $n=p^{k+1}$ (ou seja, se $m_{0}=1$ ), então $n \in D$. Se $n \neq p^{k+1}$, então $m_{0}>1$ e $p^{k+1} S$ é um subconjunto $m_{0}$-round de $G$. Como o número de divisores de $m_{0}$ é estritamente menor que o número de divisores de $n$, da hipótese de indução segue que $m_{0} \in D$. Logo, $\mathbb{Z}_{m_{0}}^{(\mathfrak{c})}$ é isomorfo a um subgrupo de $G$. Portanto, $\mathbb{Z}_{n}^{(\mathfrak{c})} \cong \mathbb{Z}_{m_{0}}^{(\mathfrak{c})} \oplus \mathbb{Z}_{p^{k+1}}^{(\mathfrak{c})}$ é isomorfo a um subgrupo de $G$, o que implica que $n \in D$.

Denotaremos por $\mathcal{H}$ o conjunto de todas as funções

$$
\begin{aligned}
h: \omega & \rightarrow \varphi[G] \subset(\mathbb{Q} / \mathbb{Z})^{\left(P_{0} \times \omega\right)} \oplus \mathbb{Q}^{\left(P_{1}\right)} \\
n & \mapsto(g(n), f(n))
\end{aligned}
$$

que satisfazem uma das seguintes condições:

(1) $\operatorname{supp} f(n) \backslash \cup_{m<n} \operatorname{supp} f(m) \neq \emptyset$, para todo $n \in \omega$;

\footnotetext{
${ }^{7}$ Com efeito, $p S$ é um subconjunto infinito de $G$, pois $S$ o é e $S$ é $n=m p$-round, o que faz com que $\varphi_{p}$ restrita a $S$ seja finita-a-um. Além disso, $p S \subset G[m]$, pois $S \subset G[n]$. Por fim, se $r$ é um divisor próprio de $m$, então $p r$ é um divisor próprio de $n$ e, portanto, $\varphi_{p r}$ restrita a $S$ é finita-a-um, o que significa que $\varphi_{r}$ restrita a $p S$ é finita-a-um. Logo, $p S$ é $m$-round.
} 
(2) $|q(f(n))|>n$, para todo $n \in \omega$;

(3) $\{|q(f(n))|: n \in \omega\}$ é limitado e $|p(f(n))|>n$, para todo $n \in \omega$;

(4) $|q(g(n))|>n$, para todo $n \in \omega$

(5) (a) $\operatorname{supp} g(n) \backslash \cup_{m<n} \operatorname{supp} g(m) \neq \emptyset$, para todo $n \in \omega$;

(b) $f(n)=0$, para todo $n \in \omega$;

(c) Existe $k \in D$ tal que $\mathrm{o}(h(n))=\mathrm{o}(g(n))=k$, para todo $n \in \omega$;

(d) $\{h(n): n \in \omega\}$ é um subconjunto $k$-round de $\varphi[G]$.

Proposição 5.2.4. Se $h: \omega \rightarrow \varphi[G] \subset(\mathbb{Q} / \mathbb{Z})^{\left(P_{0} \times \omega\right)} \oplus \mathbb{Q}^{\left(P_{1}\right)}$, então existem $j: \omega \rightarrow \omega$ estritamente crescente e $c \in \varphi[G]$ tais que a seqüência $n \mapsto(h \circ j)(n)+c$ é constante ou é de um dos tipos acima mencionados.

Demonstração. Para cada $n \in \omega$, escreveremos $h(n)=(g(n), f(n))$, onde $g: \omega \rightarrow(\mathbb{Q} / \mathbb{Z})^{\left(P_{0} \times \omega\right)}$ e $f: \omega \rightarrow \mathbb{Q}^{\left(P_{1}\right)}$. Além disso, observamos que $(0,0)=\varphi(0) \in \varphi[G]$. Há seis casos a serem considerados.

Caso 1: $\cup_{n \in \omega} \operatorname{supp} f(n)$ é infinito.

Por indução, tomemos $\left\{n_{k}: k \in \omega\right\}$ uma seqüência estritamente crescente de números naturais tal que supp $f\left(n_{k+1}\right) \backslash \cup_{l<k+1} \operatorname{supp} f\left(n_{l}\right) \neq \emptyset$. Temos que

$$
\begin{aligned}
j: \omega & \rightarrow \omega \\
k & \mapsto n_{k}
\end{aligned}
$$

é uma função estritamente crescente tal que $h \circ j$ é do tipo 1 . Neste caso, tomamos $c=(0,0)$.

Caso 2: $\cup_{n \in \omega} \operatorname{supp} f(n)$ é finito e $\{|q(f(n))|: n \in \omega\}$ é ilimitado.

Por indução, tomemos $\left\{n_{k}: k \in \omega\right\}$ uma seqüência estritamente crescente de números naturais tal que $\left|q\left(f\left(n_{k}\right)\right)\right|>k$, para todo $k \in \omega$. Temos que

$$
\begin{aligned}
j: \omega & \rightarrow \omega \\
k & \mapsto n_{k}
\end{aligned}
$$

é uma função estritamente crescente tal que $h \circ j$ é do tipo 2 . Neste caso, tomamos $c=(0,0)$. 
Caso 3: $\cup_{n \in \omega} \operatorname{supp} f(n)$ é finito, $\{|q(f(n))|: n \in \omega\}$ é limitado e $\{|p(f(n))|: n \in \omega\}$ é ilimitado. Por indução, tomemos $\left\{n_{k}: k \in \omega\right\}$ uma seqüência estritamente crescente de números naturais tal que $\left|p\left(f\left(n_{k}\right)\right)\right|>k$, para todo $k \in \omega$. Temos que

$$
\begin{aligned}
j: \omega & \rightarrow \omega \\
k & \mapsto n_{k}
\end{aligned}
$$

é uma função estritamente crescente tal que $h \circ j$ é do tipo 3 . Neste caso, tomamos $c=(0,0)$.

Caso 4: $\cup_{n \in \omega} \operatorname{supp} f(n)$ é finito, $\{|q(f(n))|: n \in \omega\}$ e $\{|p(f(n))|: n \in \omega\}$ são limitados e $\{|q(g(n))|: n \in \omega\}$ é ilimitado.

Por indução, tomemos $\left\{n_{k}: k \in \omega\right\}$ uma seqüência estritamente crescente de números naturais tal que $\left|q\left(g\left(n_{k}\right)\right)\right|>k$, para todo $k \in \omega$. Temos que

$$
\begin{aligned}
j: \omega & \rightarrow \omega \\
k & \mapsto n_{k}
\end{aligned}
$$

é uma função estritamente crescente tal que $h \circ j$ é do tipo 4 . Neste caso, tomamos $c=(0,0)$.

Caso 5: $\cup_{n \in \omega} \operatorname{supp} f(n)$ é finito, $\{|q(f(n))|: n \in \omega\},\{|p(f(n))|: n \in \omega\}$ e $\{|q(g(n))|: n \in \omega\}$ são limitados e $\cup_{n \in \omega} \operatorname{supp} g(n)$ é infinito.

Uma vez que $\cup_{n \in \omega} \operatorname{supp} g(n)$ é infinito, existe $j_{1}: \omega \rightarrow \omega$ estritamente crescente tal que $\operatorname{supp} g \circ j_{1}(n) \backslash \cup_{m<n} \operatorname{supp} g \circ j_{1}(m) \neq \emptyset$, para todo $n \in \omega$. Como $\cup_{n \in \omega} \operatorname{supp} f(n)$ é finito e tanto $\{|q(f(n))|: n \in \omega\}$ quanto $\{|p(f(n))|: n \in \omega\}$ são limitados, existe $j_{2}: \omega \rightarrow \omega$ estritamente crescente tal que $f \circ j_{1} \circ j_{2}(n)=b \in \mathbb{Q}^{\left(P_{1} \times \omega\right)}$, para todo $n \in \omega$. Temos que $\left\{\left(g \circ j_{1} \circ j_{2}(n), b\right): n \in \omega\right\}$ é um subconjunto infinito de $\varphi[G]$ e, portanto, $S=\left\{\left(g \circ j_{1} \circ j_{2}(n)-g \circ j_{1} \circ j_{2}(0), 0\right): n \in \omega\right\}$ também o é. Como $\{|q(g(n))|: n \in \omega\}$ é limitado, existe $k>1$ um número natural tal que $S \subset \varphi[G][k]$. Logo, existem $T \subset \varphi[G]$ infinito e $z \in \varphi[G]$ tais que $T+z \subset S$ e $T$ é $d$-round, para algum divisor $d$ de $k .{ }^{8}$ Seja $j_{3}: \omega \rightarrow \omega$ uma função estritamente crescente tal que $T+z=\left\{\left(g \circ j_{1} \circ j_{2} \circ j_{3}(n)-g \circ j_{1} \circ j_{2}(0), 0\right): n \in \omega\right\}$. Em outras palavras, $T=$ $\left\{\left(g \circ j_{1} \circ j_{2} \circ j_{3}(n)-g \circ j_{1} \circ j_{2}(0), 0\right)-z: n \in \omega\right\}$. Como $T$ é $d$-round, temos que $T \subset \varphi[G][d]$. Portanto, existem $j_{4}: \omega \rightarrow \omega$ uma função estritamente crescente e $r$ um divisor de $d$ tais que $\circ\left(\left(g \circ j_{1} \circ j_{2} \circ j_{3} \circ j_{4}(n)-g \circ j_{1} \circ j_{2}(0), 0\right)-z\right)=r$, para todo $n \in \omega$. Observamos que $r \in D$, uma vez que $\left\{\left(g \circ j_{1} \circ j_{2} \circ j_{3} \circ j_{4}(n)-g \circ j_{1} \circ j_{2}(0), 0\right)-z: n \in \omega\right\}$ é $r$-round. Sejam

\footnotetext{
${ }^{8}$ Observe que $z$ tem ordem finita.
} 
$c=-h \circ j_{1} \circ j_{2}(0)-z$ e $j=j_{1} \circ j_{2} \circ j_{3} \circ j_{4} \circ j_{5}$, onde $j_{5}: \omega \rightarrow \omega$ é uma função estritamente crescente tal que $\operatorname{supp}\left(g \circ j(n)-g \circ j_{1} \circ j_{2}(0)-z\right) \backslash \cup_{m<n} \operatorname{supp}\left(g \circ j(m)-g \circ j_{1} \circ j_{2}(0)-z\right) \neq \emptyset$, para todo $n \in \omega$.

Caso 6: $\cup_{n \in \omega} \operatorname{supp} f(n)$ é finito, $\{|q(f(n))|: n \in \omega\},\{|p(f(n))|: n \in \omega\}$ e $\{|q(g(n))|: n \in \omega\}$ são limitados e $\cup_{n \in \omega} \operatorname{supp} g(n)$ é finito.

Temos que $\{g(n): n \in \omega\}$ é finito, bem como $\{f(n): n \in \omega\}$. Portanto, $h$ possui uma subseqüência constante.

Proposição 5.2.5. Existe $\left\{h_{\xi}: \xi \in\left(L_{1} \cup \bigcup_{n \in D} L_{n}\right) \backslash \omega\right\}$ uma indexação de $\mathcal{H}$ tal que:

(i) $h_{\omega}(n)=\left(0, \chi_{n}\right)$, para todo $n \in \omega$.

(ii) Se $\xi \in L_{1}$, então $h_{\xi}$ é do tipo 1, 2, 3 ou 4;

(iii) Se existe $k \in D$ tal que $\xi \in L_{k}$, então $h_{\xi}$ é do tipo 5 e o $\left(h_{\xi}(n)\right)=k$, para todo $n \in \omega$;

(iv) $\cup_{n \in \omega} \operatorname{supp} h_{\xi}(n) \subset(\xi \times \omega) \cup \xi$, para todo $\xi \in\left(L_{1} \cup \bigcup_{n \in D} L_{n}\right) \backslash \omega$.

Demonstração. Observamos, primeiramente, que $|\mathcal{H}|=\mathfrak{c}^{\omega}=\mathfrak{c}$. Portanto, podemos considerar $\left\{\tilde{h}_{\xi}: \xi \in\left(L_{1} \cup \bigcup_{n \in D} L_{n}\right) \backslash \omega\right\}$ uma indexação de $\mathcal{H}$ tal que, para cada $h \in \mathcal{H}$, o conjunto

$$
A_{h}=\left\{\xi \in\left(L_{1} \cup \bigcup_{n \in D} L_{n}\right) \backslash \omega: \tilde{h}_{\xi}=h\right\}
$$

tenha cardinalidade $\mathfrak{c}$.

Fixemos $h \in \mathcal{H}$. Como $\cup_{n \in \omega} \operatorname{supp} h(n) \subset(\mathfrak{c} \times \omega) \cup \mathfrak{c}, \operatorname{cf}(\mathfrak{c})>\omega$ e $\left|\cup_{n \in \omega} \operatorname{supp} h(n)\right| \leq \omega$, existe $\alpha<\mathfrak{c}$ tal que $\alpha>\omega$ e $\cup_{n \in \omega} \operatorname{supp} h(n) \subset(\alpha \times \omega) \cup \alpha$.

Caso 1: $h$ é do tipo 1, 2, 3 ou 4 .

Se $\xi \in L_{1}$ e $\xi \geq \alpha$, façamos $h_{\xi}=\tilde{h}_{\xi}$.

Se $\xi \in L_{1}$ e $\xi<\alpha$, façamos $h_{\xi}=H$, onde $H(n)=\left(0, \chi_{n}\right)$ para todo $n \in \omega$. Observamos que $H$ é do tipo 1 e, portanto, $H \in \mathcal{H}$.

Se $\xi \in \cup_{n \in D} L_{n}$, existe um único $m \in D$ tal que $\xi \in L_{m}$. Neste caso, façamos $h_{\xi}=F_{m}$, onde $F_{m}(n)(\omega+n+1,0)$ é um elemento de $\mathbb{Q} / \mathbb{Z}$ de ordem $m, F_{m}(n)(\mu, k)=0+\mathbb{Z}$ qualquer que seja $(\mu, k) \in\left(P_{0} \times \omega\right) \backslash\{(\omega+n+1,0)\}$ e $F_{m}(n)(\theta)=0$, qualquer que seja $\theta \in P_{1}$, para todo $n \in \omega$. Temos que $F_{m}$ é do tipo 5 e, portanto, $F_{m} \in \mathcal{H}$. Além disso, o $\left(F_{m}(n)\right)=m$, para todo $n \in \omega$. 
Caso 2: $h$ é do tipo 5 .

Se $\xi \in L_{1}$, façamos $h_{\xi}=H$.

Como $h$ é do tipo 5 , existe $m \in D$ tal que o $(h(n))=m$, para todo $n \in \omega$.

Se $\xi \in L_{m}$ e $\xi \geq \alpha$, façamos $h_{\xi}=\tilde{h}_{\xi}$.

Se $\xi \in L_{m}$ e $\xi<\alpha$, façamos $h_{\xi}=F_{m}$.

Se $\xi \in L_{k}$, para algum $k \in D \backslash\{m\}$, façamos $h_{\xi}=F_{k}$.

As condições (i), (ii), (iii) e (iv) estão verificadas.

\subsection{Construindo homomorfismos de grupos}

Sejam $\left\{p_{\xi}: \xi \in\left(L_{1} \cup \bigcup_{n \in D} L_{n}\right) \backslash \omega\right\}$ uma família de ultrafiltros seletivos dois a dois incomparáveis segundo a ordem de Rudin-Keisler e $\left\{h_{\xi}: \xi \in\left(L_{1} \cup \bigcup_{n \in D} L_{n}\right) \backslash \omega\right\}$ uma indexação de $\mathcal{H}$ satisfazendo as condições (i), (ii), (iii) e (iv) da proposição 5.2.5. No que segue, escreveremos $h_{\xi}(n)=\left(g_{\xi}(n), f_{\xi}(n)\right)$, onde $g_{\xi}: \omega \rightarrow(\mathbb{Q} / \mathbb{Z})^{\left(P_{0} \times \omega\right)}$ e $f_{\xi}: \omega \rightarrow \mathbb{Q}^{\left(P_{1}\right)}$.

Proposição 5.3.1. Seja $(H, J) \in(\mathbb{Q} / \mathbb{Z})^{\left(P_{0} \times \omega\right)} \oplus \mathbb{Q}^{\left(P_{1}\right)} \backslash\{(0,0)\}$. Existe $E \in[\mathfrak{c}]^{\omega}$ tal que:

(i) $\operatorname{supp}(H, J) \subset(E \times \omega) \cup E$;

(ii) $\left|E \cap\left[\left(L_{1} \cup \bigcup_{n \in D} L_{n}\right) \backslash \omega\right]\right|=\omega$;

(iii) $\cup_{n \in \omega} \operatorname{supp} h_{\xi}(n) \subset(E \times \omega) \cup E$, qualquer que seja $\xi \in E \cap\left[\left(L_{1} \cup \bigcup_{n \in D} L_{n}\right) \backslash \omega\right]$.

Demonstração. Para cada $\xi \in \mathfrak{c} \backslash\left[\left(L_{1} \cup \bigcup_{n \in D} L_{n}\right) \backslash \omega\right]$, façamos $E(\xi)=\{\xi\} \cup \omega$. Se $\xi \in\left(L_{1} \cup \bigcup_{n \in D} L_{n}\right) \backslash \omega$, definimos indutivamente

$$
E(\xi)=\{\xi\} \cup \bigcup_{\mu \in \pi_{1}\left[\cup_{n \in \omega} \operatorname{supp} g_{\xi}(n)\right] \cup \cup_{n \in \omega} \operatorname{supp} f_{\xi}(n)} E(\mu)
$$

Fixemos $\tilde{L}_{1} \in\left[L_{1} \backslash \omega\right]^{\omega}$ e façamos

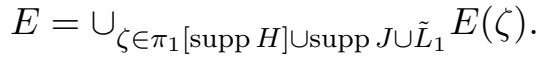

Temos que $\operatorname{supp}(H, J) \cup \tilde{L}_{1} \subset(E \times \omega) \cup E$, uma vez que $\zeta \in E(\zeta) \subset E$, qualquer que seja $\zeta \in \pi_{1}[\operatorname{supp} H] \cup \operatorname{supp} J \cup \tilde{L}_{1}$. Além disso, um argumento indutivo garante que $E(\xi) \in[\mathfrak{c}]^{\omega}$ para todo $\xi<\mathfrak{c}$ e, portanto, $E \in[\mathfrak{c}]^{\omega}$. Por fim, se $\xi \in E \cap\left[\left(L_{1} \cup \bigcup_{n \in D} L_{n}\right) \backslash \omega\right]$, então existe $\zeta \in \pi_{1}[\operatorname{supp} H] \cup \operatorname{supp} J \cup \tilde{L}_{1}$ tal que $\xi \in E(\zeta)$. Um outro argumento indutivo garante que 
se $\alpha \in E(\beta)$, então $E(\alpha) \subset E(\beta)$, quaisquer que sejam $\alpha, \beta<\mathfrak{c}$. Logo, $\pi_{1}\left[\cup_{n \in \omega} \operatorname{supp} g_{\xi}(n)\right] \cup$ $\bigcup_{n \in \omega} \operatorname{supp} f_{\xi}(n) \subset E(\xi) \subset E(\zeta) \subset E$ e, portanto, $\cup_{n \in \omega} \operatorname{supp} h_{\xi}(n) \subset(E \times \omega) \cup E$.

Lema 5.3.2. Sejam $(H, J) \in(\mathbb{Q} / \mathbb{Z})^{\left(P_{0} \times \omega\right)} \oplus \mathbb{Q}^{\left(P_{1}\right)} \backslash\{(0,0)\}$ e $E \in[\mathfrak{c}]^{\omega}$ satisfazendo as condições (i), (ii) e (iii) da proposição 5.3.1. Existe $\left\{E_{k}: k \in \omega\right\}$ uma família de subconjuntos finitos de $E$, $\left\{e_{k}: k \in \omega\right\}$ e $\left\{b_{k}: k \in \omega\right\}$ seqüências estritamente crescentes de números naturais, $\left\{r_{k}: k \in \omega\right\}$ uma seqüência de números reais positivos e $i: \omega \rightarrow E \cap\left[\left(L_{1} \cup \bigcup_{n \in D} L_{n}\right) \backslash \omega\right]$ tais que:

(i) $\operatorname{supp}(H, J) \subset\left(E_{0} \times e_{0}\right) \cup E_{0}$ ；

(ii) $E=\cup_{k \in \omega} E_{k}$;

(iii) $i(k) \in E_{k}$, para todo $k \in \omega$;

(iv) $\left(E_{k+1} \times e_{k+1}\right) \cup E_{k+1} \supset\left(E_{k} \times e_{k}\right) \cup E_{k} \cup \bigcup\left\{\operatorname{supp} h_{i(m)}\left(b_{m}\right): m \leq k\right\}$, para todo $k \in \omega$;

(v) $\left\{b_{k}: k \in i^{-1}(\{\xi\})\right\} \in p_{\xi}$, para todo $\xi \in E \cap\left[\left(L_{1} \cup \bigcup_{n \in D} L_{n}\right) \backslash \omega\right]$;

(vi) Se $h_{i(k)}$ é do tipo 1, então $\operatorname{supp} f_{i(k)}\left(b_{k}\right) \backslash E_{k} \neq \emptyset$;

(vii) Se $h_{i(k)}$ é do tipo 2, então $\left|q\left(f_{i(k)}\left(b_{k}\right)\right)\right| \cdot r_{k}>d(J) \cdot \prod_{m<k} d\left(f_{i(m)}\left(b_{m}\right)\right)$;

(viii) Se $h_{i(k)}$ é do tipo 3, então $\left|a\left(f_{i(k)}\left(b_{k}\right)\right)\right| \cdot r_{k}>4 \cdot d\left(f_{i(k)}\left(b_{k}\right)\right)$;

(ix) Se $h_{i(k)}$ é do tipo 4, então $\left|q\left(g_{i(k)}\left(b_{k}\right)\right)\right| \cdot r_{k}>d(H) \cdot \prod_{m<k} d\left(g_{i(m)}\left(b_{m}\right)\right)$;

(x) Se $h_{i(k)}$ é do tipo 5, então $\operatorname{supp} g_{i(k)}\left(b_{k}\right) \backslash\left(E_{k} \times e_{k}\right) \neq \emptyset$ e o $\left(g_{i(k)}\left(b_{k}\right)\right)=$ $\mathrm{o}\left(g_{i(k)}\left(b_{k}\right) \uparrow_{\operatorname{supp}} g_{i(k)}\left(b_{k}\right) \backslash\left(E_{k} \times e_{k}\right)\right) ;$

(xi) $r_{0}=\frac{1}{4 \cdot\|a(H, J)\|}$;

(xii) $r_{k+1}=\frac{r_{k}}{2 \cdot\left\|a\left(h_{i(k)}\left(b_{k}\right)\right)\right\|}$, para todo $k \in \omega$.

Demonstração. Consideremos $\left\{a_{n}: n \in \omega\right\}$ uma enumeração de $E$. Seja

$$
F_{0}=\pi_{1}[\operatorname{supp} H] \cup \operatorname{supp} J \cup\left\{a_{0}\right\}
$$

e tomemos $g_{0} \in \omega$ tal que $g_{0}>\max \pi_{2}[\operatorname{supp} H]$. Para cada $n \in \omega$, definimos

$$
F_{n+1}=F_{n} \cup \bigcup\left\{\pi_{1}\left[\operatorname{supp} g_{\xi}(m)\right] \cup \operatorname{supp} f_{\xi}(m): m \leq n, \xi \in F_{n} \cap\left[\left(L_{1} \cup \bigcup_{n \in D} L_{n}\right) \backslash \omega\right]\right\} \cup\left\{a_{n+1}\right\}
$$


e tomemos $g_{n+1} \in \omega$ tal que $g_{n+1}>g_{n}$ e

$$
g_{n+1}>\max \pi_{2}\left[\bigcup\left\{\operatorname{supp} g_{\xi}(n): m \leq n, \xi \in F_{n} \cap\left[\left(L_{1} \cup \bigcup_{n \in D} L_{n}\right) \backslash \omega\right]\right\}\right] .
$$

Temos que $\left\{F_{n}: n \in \omega\right\}$ é uma família de subconjuntos finitos de $E$ e $\left\{g_{n}: n \in \omega\right\}$ é uma seqüência estritamente crescente de números naturais tais que:

(1) $\operatorname{supp}(H, J) \subset\left(F_{0} \times g_{0}\right) \cup F_{0}$;

(2) $E=\cup_{n \in \omega} F_{n}$;

(3) $\left(F_{n+1} \times g_{n+1}\right) \cup F_{n+1} \supset\left(F_{n} \times g_{n}\right) \cup F_{n} \cup \bigcup\left\{\operatorname{supp} h_{\xi}(m): m \leq n, \xi \in F_{n} \cap\left[\left(L_{1} \cup \bigcup_{n \in D} L_{n}\right) \backslash \omega\right]\right\}$.

Fixemos $\xi \in E \cap\left[\left(L_{1} \cup \bigcup_{n \in D} L_{n}\right) \backslash \omega\right]$ e $n \in \omega$. Se $h_{\xi}$ é do tipo 1, definimos

$$
A_{n}^{\xi}=\left\{k \in \omega: \operatorname{supp} f_{\xi}(k) \backslash F_{n} \neq \emptyset\right\}
$$

Se $h_{\xi}$ é do tipo 2 , definimos

$$
A_{n}^{\xi}=\left\{k \in \omega:\left|q\left(f_{\xi}(k)\right)\right|>2^{n+2} \cdot X_{n-1}\right\}
$$

onde

$$
X_{m}=\|a(H, J)\| \cdot d(J) \cdot \prod_{\substack{l \leq m \\ \zeta \in F_{m} \cap\left[\left(L_{1} \cup \bigcup_{n \in D}\right.\right.}} d\left(f_{\zeta}(l)\right) \cdot \prod_{\substack{l \leq m \\ \zeta \in F_{m} \cap\left[\left(L_{1} \cup \bigcup_{n \in D}\right.\right.}}\left\|a\left(h_{\zeta}(l)\right)\right\|
$$

para todo $m \in \omega \mathrm{e}$

$$
X_{-1}=\|a(H, J)\| \cdot d(J) .
$$

Se $h_{\xi}$ é do tipo 3, então $\left|a\left(f_{\xi}(n)\right)\right|>n$, para cada $n \in \omega$ e $\left\{\left|q\left(f_{\xi}(n)\right)\right|: n \in \omega\right\}$ é limitado. Tomemos $M_{\xi} \in \omega$ tal que $\left|q\left(f_{\xi}(n)\right)\right| \leq M_{\xi}$, para todo $n \in \omega$ e definimos

$$
A_{n}^{\xi}=\left\{k \in \omega:\left|a\left(f_{\xi}(k)\right)\right|>2^{n+4} \cdot M_{\xi} ! \cdot Y_{n-1}\right\}
$$

onde

$$
Y_{m}=\|a(H, J)\| \cdot \prod_{\substack{l \leq m \\ \zeta \in F_{m} \cap\left[\left(L_{1} \cup \bigcup_{n \in D} L_{n}\right) \backslash \omega\right]}}\left\|a\left(h_{\zeta}(l)\right)\right\|
$$


para todo $m \in \omega \mathrm{e}$

$$
Y_{-1}=\|a(H, J)\|
$$

Se $h_{\xi}$ é do tipo 4, definimos

$$
A_{n}^{\xi}=\left\{k \in \omega:\left|q\left(g_{\xi}(k)\right)\right|>2^{n+2} \cdot Z_{n-1}\right\}
$$

onde

$$
\left.Z_{m}=\|a(H, J)\| \cdot d(H) \cdot \prod_{\substack{l \leq m \\ \zeta \in F_{m} \cap\left[\left(L_{1} \cup \bigcup_{n \in D} L_{n}\right) \backslash \omega\right]}} d\left(g_{\zeta}(l)\right) \cdot \prod_{\substack{l \leq m \\ \zeta \in F_{m} \cap\left[\left(L_{1} \cup \bigcup_{n \in D}\right.\right.}}\left\|a\left(h_{\zeta}(l)\right)\right\| \omega\right] \|
$$

para todo $m \in \omega \mathrm{e}$

$$
Z_{-1}=\|a(H, J)\| \cdot d(H)
$$

Se $h_{\xi}$ é do tipo 5, definimos

$$
A_{n}^{\xi}=\left\{k \in \omega: \operatorname{supp} g_{\xi}(k) \backslash\left(F_{n} \times g_{n}\right) \neq \emptyset\right\} \cap\left\{k \in \omega: \mathrm{o}\left(g_{\xi}(k) \uparrow_{\operatorname{supp} g_{\xi}(k) \backslash\left(F_{n} \times g_{n}\right)}\right)=\mathrm{o}\left(g_{\xi}(k)\right)\right\} .
$$

Observamos que $A_{n}^{\xi}$ é um subconjunto cofinito de $\omega$, quaisquer que sejam $n \in \omega$ e $\xi \in\left[E \cap\left(L_{1} \cup \bigcup_{n \in D} L_{n}\right)\right] \backslash \omega .^{9} \quad$ Logo, para cada $\xi \in\left[E \cap\left(L_{1} \cup \bigcup_{n \in D} L_{n}\right)\right] \backslash \omega$, temos que $\left\{A_{n}^{\xi}: n \in \omega\right\} \subset p_{\xi}$, uma vez que $p_{\xi}$ é um ultrafiltro livre sobre $\omega$. Da seletividade de $p_{\xi}$ decorre que existe uma seqüência $\left\{a_{n}^{\xi}: n \in \omega\right\} \in p_{\xi}$ tal que $a_{n}^{\xi} \in A_{n}^{\xi}$ e $n<a_{n}^{\xi}$, para cada $n \in \omega$.

Do lema 1.5.4 segue que existe $\left\{I_{\xi}: \xi \in\left[E \cap\left(L_{1} \cup \bigcup_{n \in D} L_{n}\right)\right] \backslash \omega\right\}$ uma família de subconjuntos de $\omega$ dois a dois disjuntos tal que:

(a) $\left\{a_{n}^{\xi}: n \in I_{\xi}\right\} \in p_{\xi}$, para cada $\xi \in\left[E \cap\left(L_{1} \cup \bigcup_{n \in D} L_{n}\right)\right] \backslash \omega$;

(b) $\left\{\left[n, a_{n}^{\xi}\right]: n \in I_{\xi}, \xi \in\left[E \cap\left(L_{1} \cup \bigcup_{n \in D} L_{n}\right)\right] \backslash \omega\right\}$ é uma família de intervalos de $\omega$ dois a dois disjuntos.

Para cada $\xi \in\left[E \cap\left(L_{1} \cup \bigcup_{n \in D} L_{n}\right)\right] \backslash \omega$, seja

\footnotetext{
${ }^{9}$ Sejam $\xi \in\left[E \cap\left(L_{1} \cup \bigcup_{n \in D} L_{n}\right)\right] \backslash \omega$ e $n \in \omega$. Suponhamos que $h_{\xi}$ seja do tipo 5 . Temos que $\left\{k \in \omega: \operatorname{supp} g_{\xi}(k) \backslash\left(F_{n} \times g_{n}\right) \neq \emptyset\right\}$ é um subconjunto cofinito de $\omega$. Como $h_{\xi}$ é do tipo 5 , existe $d \in D$ tal que $\left\{h_{\xi}(k): k \in \omega\right\}$ é um subconjunto $d$-round de $\varphi[G]$ e o $\left(h_{\xi}(k)\right)=\mathrm{o}\left(g_{\xi}(k)\right)=d$, para todo $k \in \omega$. Por absurdo, suponhamos que $\left\{k \in \omega: \mathrm{o}\left(g_{\xi}(k) \uparrow_{\operatorname{supp}} g_{\xi}(k) \backslash\left(F_{n} \times g_{n}\right)\right)=\mathrm{o}\left(g_{\xi}(k)\right)\right\}$ não seja um subconjunto cofinito de $\omega$. Tomemos $r$ um divisor próprio de $d$ e $I \in[\omega]^{\omega}$ tais que o $\left(g_{\xi}(k)\left\lceil\operatorname{supp} g_{\xi}(k) \backslash\left(F_{n} \times g_{n}\right)\right)=r\right.$, para todo $k \in I$. Note que $\operatorname{supp}\left(r \cdot h_{\xi}(k)\right) \subset F_{n} \times g_{n}$, para todo $k \in I$. Temos que $\left\{r \cdot h_{\xi}(k): k \in I\right\} \subset \oplus_{(\zeta, m) \in F_{n} \times g_{n}} G_{(\zeta, m)}$, onde $G_{(\zeta, m)}=(\mathbb{Q} / \mathbb{Z})[d / r]$ e, portanto, $\left\{r \cdot h_{\xi}(k): k \in I\right\}$ é um conjunto finito, o que contradiz o fato de $\left\{h_{\xi}(k): k \in \omega\right\}$ ser $d$-round.
} 
(c) $N_{\xi}=\min \left\{n \in \omega: \xi \in F_{n}\right\}$.

Podemos supor, sem perda de generalidade, que

(d) $N_{\xi}<n$, para todo $n \in I_{\xi}$.

Consideremos $\left\{n_{k}: k \in \omega\right\}$ uma enumeração estritamente crescente de $\dot{\cup}_{\xi \in E \cap\left[\left(L_{1} \cup \cup_{n \in D} L_{n}\right) \backslash \omega\right]} I_{\xi}$. Seja

$$
\begin{aligned}
i: \omega & \rightarrow E \cap\left[\left(L_{1} \cup \bigcup_{n \in D} L_{n}\right) \backslash \omega\right] \\
k & \mapsto i(k)
\end{aligned}
$$

onde $i(k)$ é o único elemento de $E \cap\left[\left(L_{1} \cup \bigcup_{n \in D} L_{n}\right) \backslash \omega\right]$ tal que $n_{k} \in I_{i(k)}$.

Para cada $k \in \omega$, façamos $b_{k}=a_{n_{k}}^{i(k)}, E_{k}=F_{n_{k}}, e_{k}=g_{n_{k}}$ e definamos $r_{k}$ segundo os ítens (xi) e (xii) do enunciado deste lema. Resta mostrar que as condições (i)-(x) estão satisfeitas.

(i) Como $0 \leq n_{0}$, temos que $E_{0}=F_{n_{0}} \supset F_{0} \supset \pi_{1}[\operatorname{supp} H] \cup \operatorname{supp} J$ e $e_{0}=g_{n_{0}}>g_{0}>$ $\max \pi_{2}[\operatorname{supp} H] . \operatorname{Logo}, \operatorname{supp}(H, J) \subset\left(E_{0} \times e_{0}\right) \cup E_{0}$.

(ii) $\cup_{k \in \omega} E_{k}=\cup_{k \in \omega} F_{n_{k}}=E$.

(iii) Seja $k \in \omega$. Temos que $n_{k} \in I_{i(k)}$. De (d) segue que $N_{i(k)}<n_{k}$. De (c) e (3) vem que $i(k) \in F_{N_{i(k)}} \subset F_{n_{k}}=E_{k}$.

(iv) De (3) vem que $E_{k} \subset E_{k+1}$ e $\left(E_{k} \times e_{k}\right) \subset\left(E_{k+1} \times e_{k+1}\right)$, pois $\left\{n_{k}: k \in \omega\right\}$ é uma enumeração estritamente crescente de $\cup_{\xi \in E \cap\left[\left(L_{1} \cup \cup_{n \in D} L_{n}\right) \backslash \omega\right]} I_{\xi}$. Resta mostrar que $\operatorname{supp} h_{i(m)}\left(b_{m}\right) \subset\left(E_{k+1} \times e_{k+1}\right) \cup E_{k+1}$, para todo $m \leq k$. Temos que $\left(E_{k+1} \times e_{k+1}\right) \cup E_{k+1}=$ $\left(F_{n_{k+1}} \times g_{n_{k+1}}\right) \cup F_{n_{k+1}}$ e de $(3)$ decorre que

$\left(F_{n_{k+1}} \times g_{n_{k+1}}\right) \cup F_{n_{k+1}} \supset \bigcup\left\{\operatorname{supp} h_{\xi}(m): m \leq n_{k+1}-1, \xi \in F_{n_{k+1}-1} \cap\left[\left(L_{1} \cup \bigcup_{n \in D} L_{n}\right) \backslash \omega\right]\right\}$.

Por (iii), $i(m) \in E_{m} \subset E_{k}=F_{n_{k}} \subset F_{n_{k+1}-1}$, para todo $m \leq k$. Além disso, $i(m) \in\left[\left(L_{1} \cup \bigcup_{n \in D} L_{n}\right) \backslash \omega\right]$. Por (b), $b_{m}=a_{n_{m}}^{i(m)} \leq n_{k+1}-1$, para todo $m \leq k$. Logo, $\operatorname{supp} h_{i(m)}\left(b_{m}\right) \subset\left(E_{k+1} \times e_{k+1}\right) \cup E_{k+1}$, para todo $m \leq k$.

(v) Seja $\xi \in E \cap\left[\left(L_{1} \cup \bigcup_{n \in D} L_{n}\right) \backslash \omega\right]$. Temos que $\left\{b_{k}: k \in i^{-1}(\{\xi\})\right\}=\left\{a_{n_{k}}^{i(k)}: k \in i^{-1}(\{\xi\})\right\}=$ $\left\{a_{n}^{\xi}: n \in I_{\xi}\right\} \in p_{\xi}$, por (a). 
(vi) Suponhamos que $h_{i(k)}$ seja do tipo 1. Temos que $b_{k}=a_{n_{k}}^{i(k)} \in A_{n_{k}}^{i(k)}$. Da definição de $A_{n_{k}}^{i(k)}$ vem que

$$
\operatorname{supp} f_{i(k)}\left(b_{k}\right) \backslash E_{k}=\operatorname{supp} f_{i(k)}\left(b_{k}\right) \backslash F_{n_{k}} \neq \emptyset \text {. }
$$

(vii) Suponhamos que $h_{i(k)}$ seja do tipo 2. Temos que $b_{k}=a_{n_{k}}^{i(k)} \in A_{n_{k}}^{i(k)}$. Da definição de $A_{n_{k}}^{i(k)}$ vem que

$$
\left|q\left(f_{i(k)}\left(b_{k}\right)\right)\right|>2^{n_{k}+2} \cdot X_{n_{k}-1}
$$

onde

$$
X_{n_{k}-1}=\|a(H, J)\| \cdot d(J) \cdot \prod_{\substack{m \leq n_{k}-1 \\ \xi \in F_{n_{k}-1} \cap\left[\left(L_{1} \cup \bigcup_{n \in D} L_{n}\right) \backslash \omega\right]}} d\left(f_{\xi}(m)\right) . \prod_{\substack{m \leq n_{k}-1 \\ \xi \in F_{n_{k}-1} \cap\left[\left(L_{1} \cup \bigcup_{n \in D} L_{n}\right) \backslash \omega\right]}}\left\|a\left(h_{\xi}(m)\right)\right\| .
$$

Observamos que se $m<k$, então $n_{m}<n_{k}$ e, portanto, $i(m) \in E_{m}=F_{n_{m}} \subset F_{n_{k-1}} \subset F_{n_{k}-1}$. Como $b_{m}=a_{n_{m}}^{i(m)}$ e $\left[n_{m}, a_{n_{m}}^{i(m)}\right] \cap\left[n_{k}, a_{n_{k}}^{i(k)}\right]=\emptyset$, temos que $b_{m}<n_{k}$ e, portanto, $b_{m} \leq n_{k}-1$. Uma vez que

$$
r_{k}=\frac{1}{2^{k+2} \cdot\|a(H, J)\| \cdot \prod_{m<k}\left\|a\left(h_{i(m)}\left(b_{m}\right)\right)\right\|}
$$

concluímos que

$$
\left|q\left(f_{i(k)}\left(b_{k}\right)\right)\right| \cdot r_{k}>d(J) \cdot \prod_{m<k} d\left(f_{i(m)}\left(b_{m}\right)\right)
$$

(viii) Suponhamos que $h_{i(k)}$ seja do tipo 3. Um argumento análogo ao utilizado em (vii) nos permite concluir que

$$
\left|a\left(f_{i(k)}\left(b_{k}\right)\right)\right| \cdot r_{k}>4 \cdot M_{i(k)} ! \geq 4 \cdot d\left(f_{i(k)}\left(b_{k}\right)\right) .
$$

(ix) Suponhamos que $h_{i(k)}$ seja do tipo 4. Um argumento análogo ao utilizado em (vii) nos permite concluir que

$$
\left|q\left(g_{i(k)}\left(b_{k}\right)\right)\right| \cdot r_{k}>d(H) \cdot \prod_{m<k} d\left(g_{i(m)}\left(b_{m}\right)\right)
$$

(x) Suponhamos que $h_{i(k)}$ seja do tipo 5. Temos que $b_{k}=a_{n_{k}}^{i(k)} \in A_{n_{k}}^{i(k)}$. Da definição de $A_{n_{k}}^{i(k)}$ vem que

$$
\operatorname{supp} g_{i(k)}\left(b_{k}\right) \backslash\left(E_{k} \times e_{k}\right)=\operatorname{supp} g_{i(k)}\left(b_{k}\right) \backslash\left(F_{n_{k}} \times g_{n_{k}}\right) \neq \emptyset
$$


e

$$
\mathrm{o}\left(g_{i(k)}\left(b_{k}\right)\left\lceil_{\operatorname{supp}} g_{i(k)}\left(b_{k}\right) \backslash\left(E_{k} \times e_{k}\right)\right)=\mathrm{o}\left(g_{i(k)}\left(b_{k}\right)\left\lceil_{\operatorname{supp}} g_{i(k)}\left(b_{k}\right) \backslash\left(F_{n_{k}} \times g_{n_{k}}\right)\right)=\mathrm{o}\left(g_{i(k)}\left(b_{k}\right)\right)\right. \text {. }\right.
$$

Lema 5.3.3. Sejam $(H, J) \in(\mathbb{Q} / \mathbb{Z})^{\left(P_{0} \times \omega\right)} \oplus \mathbb{Q}^{\left(P_{1}\right)} \backslash\{(0,0)\}$ e $E \in[\mathfrak{c}]^{\omega}$ satisfazendo as condições

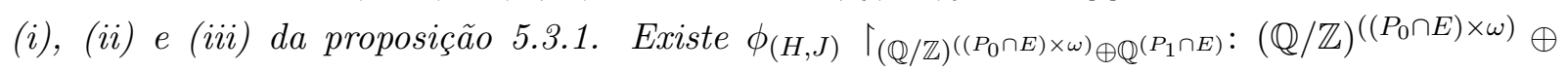
$\mathbb{Q}^{\left(P_{1} \cap E\right)} \rightarrow \mathbb{T}$ um homomorfismo de grupos com as seguintes propriedades:

(i) $\phi_{(H, J)} \uparrow_{(\mathbb{Q} / \mathbb{Z})}\left(\left(P_{0} \cap E\right) \times \omega\right) \oplus \mathbb{Q}^{\left(P_{1} \cap E\right)}(H, J) \neq 0+\mathbb{Z}$;

(ii) $\phi_{(H, J)} \Upsilon_{(\mathbb{Q} / \mathbb{Z})^{\left(\left(P_{0} \cap E\right) \times \omega\right)} \oplus \mathbb{Q}^{\left(P_{1} \cap E\right)}}\left(0, \chi_{\xi}\right)=p_{\xi}-\lim \left\{\phi_{(H, J)} \Upsilon_{(\mathbb{Q} / \mathbb{Z})^{\left(\left(P_{0} \cap E\right) \times \omega\right)} \oplus \mathbb{Q}^{\left(P_{1} \cap E\right)}}\left(h_{\xi}(n)\right)\right.$ : $n \in \omega\}$, para cada $\xi \in\left(E \cap L_{1}\right) \backslash \omega$;

(iii) $\phi_{(H, J)} \Upsilon_{(\mathbb{Q} / \mathbb{Z})^{\left(\left(P_{0} \cap E\right) \times \omega\right)} \oplus \mathbb{Q}^{\left(P_{1} \cap E\right)}}\left(y_{\xi}, 0\right)=p_{\xi}-\lim \left\{\phi_{(H, J)} \Upsilon_{(\mathbb{Q} / \mathbb{Z})^{\left(\left(P_{0} \cap E\right) \times \omega\right)} \oplus \mathbb{Q}^{\left(P_{1} \cap E\right)}}\left(h_{\xi}(n)\right): n \in\right.$ $\omega\}$, para cada $\xi \in\left(E \cap \bigcup_{n \in D} L_{n}\right) \backslash \omega .^{10}$

Demonstração. Consideremos $\left\{E_{n}: n \in \omega\right\},\left\{e_{n}: n \in \omega\right\},\left\{b_{n}: n \in \omega\right\},\left\{r_{n}: n \in \omega\right\}$ e $i: \omega \rightarrow\left[E \cap\left(L_{1} \cup \bigcup_{n \in D} L_{n}\right)\right] \backslash \omega$ de acordo com o lema 5.3.2.

Se $H=0$, façamos $\hat{\phi}(H)=0+\mathbb{Z}$. Se $H \neq 0$, seja $\hat{\phi}(H)$ um elemento não nulo de $\mathbb{T}$ tal que $\mathrm{o}(\hat{\phi}(H)) \mid \mathrm{o}(H)$. Em ambos os casos, estendemos $\hat{\phi}$ a um homomorfismo de grupos de $\langle H\rangle \mathrm{em}$ $\mathbb{T}$. Dados $(\zeta, m) \in\left(P_{0} \cap E\right) \times \omega$ e $k \in \mathbb{N} \backslash\{0\}$, definimos $\Lambda_{(\zeta, m), k}:\left(P_{0} \cap E\right) \times \omega \rightarrow \mathbb{Q} / \mathbb{Z}$ por

$$
\Lambda_{(\zeta, m), k}(\mu, l)=\left\{\begin{array}{ccc}
\frac{1}{k}+\mathbb{Z} & \text { se } & (\mu, l)=(\zeta, m) \\
0+\mathbb{Z} & \text { se } & (\mu, l) \neq(\zeta, m) .
\end{array}\right.
$$

Seja

$$
G_{0}=\left\langle\left\{\Lambda_{(\zeta, m), c_{0}}:(\zeta, m) \in E_{0} \times e_{0}\right\}\right\rangle
$$

onde

$$
c_{0}=\left\{\begin{array}{lll}
c_{-1} & \text { se } & i(0) \notin \cup_{n \in D} L_{n} \\
c_{-1} \cdot d\left(y_{i(0)}\right) & \text { se } & i(0) \in \cup_{n \in D} L_{n}
\end{array}\right.
$$

e

$$
c_{-1}=d(H)
$$

\footnotetext{
${ }^{10}$ Estamos considerando $(\mathbb{Q} / \mathbb{Z})^{\left(\left(P_{0} \cap E\right) \times \omega\right)} \oplus \mathbb{Q}^{\left(P_{1} \cap E\right)} \subset(\mathbb{Q} / \mathbb{Z})^{\left(P_{0} \times \omega\right)} \oplus \mathbb{Q}^{\left(P_{1}\right)}$.
} 
Como $\mathbb{T}$ é um grupo divisível e $\langle H\rangle$ é um subgrupo de $G_{0}$, podemos estender $\hat{\phi}$ a um homomorfismo de grupos $\phi\left\lceil_{G_{0}}: G_{0} \rightarrow \mathbb{T}\right.$.

Se $J=0$, tomemos $\psi_{0}^{*}(\xi)$ um arco aberto de $\mathbb{T}$ com diâmetro

$$
\delta\left(\psi_{0}^{*}(\xi)\right)=\frac{r_{0}}{d(J)}
$$

e façamos $\psi_{0}(\xi)=d(J) \cdot \psi_{0}^{*}(\xi)$, para todo $\xi \in E_{0} \cap P_{1}$.

Suponhamos $J \neq 0$. Para cada $\xi \in E_{0} \cap P_{1}$, tomemos $s_{\xi} \in \mathbb{R}$ tal que

$$
\sum_{\xi \in \operatorname{supp} J} J(\xi) \cdot s_{\xi}=\frac{1}{2}-x
$$

onde $x \in\left[0,1\left[\right.\right.$ é tal que $x+\mathbb{Z}=\phi \uparrow_{G_{0}}(H)$ e façamos

$$
t_{\xi}=\frac{1}{d(J)} \cdot s_{\xi}+\mathbb{Z}
$$

Temos que

$$
\sum_{\xi \in \operatorname{supp} J} a(J, \xi) \cdot t_{\xi}=\left(\frac{1}{2}+\mathbb{Z}\right)-\phi \uparrow_{G_{0}}(H)
$$

Seja $\psi_{0}^{*}(\xi)$ o arco aberto de $\mathbb{T}$ centrado em $t_{\xi}$ com diâmetro

$$
\delta\left(\psi_{0}^{*}(\xi)\right)=\frac{r_{0}}{d(J)}
$$

Seja, também, $\psi_{0}(\xi)=d(J) \cdot \psi_{0}^{*}(\xi)$. Claramente, $\delta\left(\psi_{0}(\xi)\right)=r_{0}$. Uma vez que

$$
\frac{1}{2}+\mathbb{Z} \in \phi\left\lceil_{G_{0}}(H)+\sum_{\xi \in \operatorname{supp} J} a(J, \xi) \cdot \psi_{0}^{*}(\xi)\right.
$$

e

$$
\delta\left(\phi\left\lceil_{G_{0}}(H)+\sum_{\xi \in \operatorname{supp} J} a(J, \xi) \cdot \psi_{0}^{*}(\xi)\right)=\delta\left(\sum_{\xi \in \operatorname{supp} J} a(J, \xi) \cdot \psi_{0}^{*}(\xi)\right) \leq \sum_{\xi \in \operatorname{supp} J}|a(J, \xi)| \cdot \frac{r_{0}}{d(J)} \leq \frac{1}{4}\right.
$$

concluímos que

$$
0+\mathbb{Z} \notin \phi \uparrow_{G_{0}}(H)+\sum_{\xi \in \operatorname{supp} J} a(J, \xi) \cdot \psi_{0}^{*}(\xi) .
$$

Por fim, se $\xi \in\left(E \backslash E_{0}\right) \cap P_{1}$, façamos $\psi_{0}(\xi)=\mathbb{T}$. 
Seja $n \in \omega$. Suponhamos definidas $\psi_{n}: E \cap P_{1} \rightarrow \mathcal{B}$ e $\psi_{n}^{*}: E_{n} \cap P_{1} \rightarrow \mathcal{B}$. Suponhamos, também, definido $\phi \Gamma_{G_{n}}: G_{n} \rightarrow \mathbb{T}$ um homomorfismo de grupos, onde

$$
G_{n}=\left\langle\left\{\Lambda_{(\zeta, m), c_{n} \cdot \prod_{k<n} d\left(g_{i(k)}\left(b_{k}\right)\right)}:(\zeta, m) \in E_{n} \times e_{n}\right\}\right\rangle
$$

e

$$
c_{n}=\left\{\begin{array}{lll}
c_{n-1} & \text { se } & i(n) \notin \cup_{m \in D} L_{m} \\
c_{n-1} \cdot d\left(y_{i(n)}\right) & \text { se } & i(n) \in \cup_{m \in D} L_{m}
\end{array}\right.
$$

Vamos definir $\psi_{n+1}: E \cap P_{1} \rightarrow \mathcal{B}, \psi_{n+1}^{*}: E_{n+1} \cap P_{1} \rightarrow \mathcal{B}$ e estender $\phi \uparrow_{G_{n}}$ a um homomorfismo de grupos $\psi \uparrow_{G_{n+1}}: G_{n+1} \rightarrow \mathbb{T}$, onde

$$
G_{n+1}=\left\langle\left\{\Lambda_{(\zeta, m), c_{n+1} \cdot \prod_{k<n+1} d\left(g_{i(k)}\left(b_{k}\right)\right)}:(\zeta, m) \in E_{n+1} \times e_{n+1}\right\}\right\rangle
$$

com

$$
c_{n+1}=\left\{\begin{array}{lll}
c_{n} & \text { se } & i(n+1) \notin \cup_{m \in D} L_{m} \\
c_{n} \cdot d\left(y_{i(n+1)}\right) & \text { se } & i(n+1) \in \cup_{m \in D} L_{m}
\end{array}\right.
$$

satisfazendo as seguintes condições:

(1) Se $\xi \in\left(E \backslash E_{n+1}\right) \cap P_{1}$, então $\psi_{n+1}(\xi)=\mathbb{T}$;

(2) Se $\xi \in\left(E_{n+1} \backslash E_{n}\right) \cap P_{1}$, então $\psi_{n+1}^{*}(\xi)$ é tal que:

(a) $d(J) \cdot \prod_{m<n+1} d\left(f_{i(m)}\left(b_{m}\right)\right) \cdot \overline{\psi_{n+1}^{*}(\xi)} \subset \psi_{n}(\xi)$;

(b) $\delta\left(\psi_{n+1}^{*}(\xi)\right)=\frac{r_{n+1}}{d(J) \cdot \prod_{m<n+1} d\left(f_{i(m)}\left(b_{m}\right)\right)}$.

Neste caso, façamos

$$
\psi_{n+1}(\xi)=d(J) \cdot \prod_{m<n+1} d\left(f_{i(m)}\left(b_{m}\right)\right) \cdot \psi_{n+1}^{*}(\xi) .
$$

(3) Se $\xi \in E_{n} \cap P_{1}$, então $\psi_{n+1}^{*}(\xi)$ é tal que:

(a) $d\left(f_{i(n)}\left(b_{n}\right)\right) \cdot \overline{\psi_{n+1}^{*}(\xi)} \subset \psi_{n}^{*}(\xi) ;$

(b) $\delta\left(\psi_{n+1}^{*}(\xi)\right)=\frac{r_{n+1}}{d(J) \cdot \prod_{m<n+1} d\left(f_{i(m)}\left(b_{m}\right)\right)}$. 
Neste caso, façamos

$$
\psi_{n+1}(\xi)=d(J) \cdot \prod_{m<n+1} d\left(f_{i(m)}\left(b_{m}\right)\right) \cdot \psi_{n+1}^{*}(\xi) .
$$

(4) Se $h_{i(n)}$ for do tipo 1, 2, 3 ou 4 , então

$$
\psi_{n}(i(n)) \cap\left(\phi \Gamma_{G_{n+1}}\left(g_{i(n)}\left(b_{n}\right)\right)+\sum_{\mu \in \operatorname{supp} f_{i(n)}\left(b_{n}\right)} a\left(f_{i(n)}\left(b_{n}\right), \mu\right) \cdot d(J) \cdot \prod_{m<n} d\left(f_{i(m)}\left(b_{m}\right)\right) \cdot \psi_{n+1}^{*}(\mu)\right) \neq \emptyset
$$

(5) Se $h_{i(n)}$ for do tipo 5 , então $\phi\left\lceil_{G_{n+1}}\left(g_{i(n)}\left(b_{n}\right)\right)=\sum_{(\zeta, p) \in \operatorname{supp} y_{i(n)} \cap\left(E_{n} \times e_{n}\right)} a\left(y_{i(n)},(\zeta, p)\right)\right.$. $c_{n-1} \cdot \prod_{m<n} d\left(g_{i(m)}\left(b_{m}\right)\right) \cdot \phi\left\lceil_{G_{n}}\left(\Lambda_{(\zeta, p), c_{n} \cdot \prod_{m<n} d\left(g_{i(m)}\left(b_{m}\right)\right)}\right)\right.$.

Se $\xi \in\left(E \backslash E_{n+1}\right) \cap P_{1}$, façamos $\psi_{n+1}(\xi)=\mathbb{T}$. Se $\xi \in\left[\left(E_{n+1} \backslash E_{n}\right) \backslash \operatorname{supp} f_{i(n)}\left(b_{n}\right)\right] \cap P_{1}$, então $\psi_{n}(\xi)=\mathbb{T}$. Definimos $\psi_{n+1}^{*}(\xi)$ como sendo um elemento de $\mathcal{B}$ com diâmetro

$$
\delta\left(\psi_{n+1}^{*}(\xi)\right)=\frac{r_{n+1}}{d(J) \cdot \prod_{m<n+1} d\left(f_{i(m)}\left(b_{m}\right)\right)}
$$

e façamos

$$
\psi_{n+1}(\xi)=d(J) \cdot \prod_{m<n+1} d\left(f_{i(m)}\left(b_{m}\right)\right) \cdot \psi_{n+1}^{*}(\xi) .
$$

Se $\xi \in\left(E_{n} \backslash \operatorname{supp} f_{i(n)}\left(b_{n}\right)\right) \cap P_{1}$, fixemos uma raiz $d\left(f_{i(n)}\left(b_{n}\right)\right)$-ésima do ponto médio de $\psi_{n}^{*}(\xi)$. Definimos $\psi_{n+1}^{*}(\xi)$ como sendo o arco aberto de $\mathbb{T}$ centrado na raiz em questão com diâmetro

$$
\delta\left(\psi_{n+1}^{*}(\xi)\right)=\frac{r_{n+1}}{d(J) \cdot \prod_{m<n+1} d\left(f_{i(m)}\left(b_{m}\right)\right)}
$$

e façamos

$$
\psi_{n+1}(\xi)=d(J) \cdot \prod_{m<n+1} d\left(f_{i(m)}\left(b_{m}\right)\right) \cdot \psi_{n+1}^{*}(\xi)
$$

Vamos, agora, definir $\psi_{n+1}^{*}(\xi)$ e $\psi_{n+1}(\xi)$ para $\xi \in \operatorname{supp} f_{i(n)}\left(b_{n}\right)$ e estender $\phi \uparrow_{G_{n}}$ a $\phi \uparrow_{G_{n+1}}$.

Caso 1: $h_{i(n)}$ é do tipo 1.

Como $\mathbb{T}$ é divisível e $G_{n}$ é um subgrupo de $G_{n+1}$, podemos estender $\phi \Upsilon_{G_{n}}$ a um homomorfismo de grupos $\phi\left\lceil_{G_{n+1}}: G_{n+1} \rightarrow \mathbb{T}\right.$. Fixemos $\alpha \in \operatorname{supp} f_{i(n)}\left(b_{n}\right) \backslash E_{n}$. Aplicando o lema 3.2 .3 para $P_{1}, d(J) \cdot \prod_{m<n} d\left(f_{i(m)}\left(b_{m}\right)\right), r_{n}, \psi_{n}(i(n))-\phi\left\lceil_{G_{n+1}}\left(g_{i(n)}\left(b_{n}\right)\right)\right.$, 
$E_{n} \cap \operatorname{supp} f_{i(n)}\left(b_{n}\right), f_{i(n)}\left(b_{n}\right), \alpha$ e $\psi_{n}^{*}$, obtemos $\tilde{\psi}: \operatorname{supp} f_{i(n)}\left(b_{n}\right) \rightarrow \mathbb{T}$ satisfazendo as condições (i), (ii), (iii) e (iv) do lema 3.2.3. Façamos $\psi_{n+1}^{*}(\xi)=\tilde{\psi}(\xi)$, para todo $\xi \in \operatorname{supp} f_{i(n)}\left(b_{n}\right)$. As condições (1), (2), (3) e (4) estão satisfeitas.

Caso 2: $h_{i(n)}$ é do tipo 2 .

Como $\mathbb{T}$ é divisível e $G_{n}$ é um subgrupo de $G_{n+1}$, podemos estender $\phi \Upsilon_{G_{n}}$ a um homomorfismo de grupos $\phi \uparrow_{G_{n+1}}: G_{n+1} \rightarrow \mathbb{T}$. Tomemos $\alpha \in \operatorname{supp} f_{i(n)}\left(b_{n}\right)$ tal que $q\left(f_{i(n)}\left(b_{n}\right), \alpha\right) \cdot r_{n}>d(J) \cdot \prod_{m<n} d\left(f_{i(m)}\left(b_{m}\right)\right)$. Podemos supor que $\alpha \in E_{n}$ pois, do contrário, os argumentos utilizados no caso 1 podem ser repetidos aqui. Aplicando o lema 3.2.4 para $P_{1}, d(J) \cdot \prod_{m<n} d\left(f_{i(m)}\left(b_{m}\right)\right), r_{n}, \psi_{n}(i(n))-\phi \Gamma_{G_{n+1}}\left(g_{i(n)}\left(b_{n}\right)\right), E_{n} \cap \operatorname{supp} f_{i(n)}\left(b_{n}\right), f_{i(n)}\left(b_{n}\right)$, $\alpha$ e $\psi_{n}^{*}$, obtemos $\tilde{\psi}: \operatorname{supp} f_{i(n)}\left(b_{n}\right) \rightarrow \mathbb{T}$ satisfazendo as condições (i), (ii), (iii) e (iv) do lema 3.2.4. Façamos $\psi_{n+1}^{*}(\xi)=\tilde{\psi}(\xi)$, para todo $\xi \in \operatorname{supp} f_{i(n)}\left(b_{n}\right)$. As condições $(1),(2)$, (3) e (4) estão satisfeitas.

Caso 3: $h_{i(n)}$ é do tipo 3 .

Como $\mathbb{T}$ é divisível e $G_{n} \subset G_{n+1}$, podemos estender $\phi$ a um homomorfismo de grupos de $G_{n+1}$ em $\mathbb{T}$. Tomemos $\alpha \in \operatorname{supp} f_{i(n)}\left(b_{n}\right)$ tal que $\left|a\left(f_{i(n)}\left(b_{n}\right), \alpha\right)\right| \cdot r_{n}>4 \cdot d\left(f_{i(n)}\left(b_{n}\right)\right)$. Novamente, podemos supor que $\alpha \in E_{n}$. Aplicando o lema 3.2 .5 para $P_{1}, d(J)$. $\prod_{m<n} d\left(f_{i(m)}\left(b_{m}\right)\right), r_{n}, \psi_{n}(i(n))-\phi\left\lceil_{G_{n+1}}\left(g_{i(n)}\left(b_{n}\right)\right), E_{n} \cap \operatorname{supp} f_{i(n)}\left(b_{n}\right), f_{i(n)}\left(b_{n}\right), \alpha\right.$ e $\psi_{n}^{*}$, obtemos $\tilde{\psi}: \operatorname{supp} f_{i(n)}\left(b_{n}\right) \rightarrow \mathbb{T}$ satisfazendo as condições (i), (ii), (iii) e (iv) do lema 3.2.5. Façamos $\psi_{n+1}^{*}(\xi)=\tilde{\psi}(\xi)$, para todo $\xi \in \operatorname{supp} f_{i(n)}\left(b_{n}\right)$. As condições $(1),(2),(3)$ e (4) estão satisfeitas.

Caso 4: $h_{i(n)}$ é do tipo 4 .

Se $\xi \in\left(E_{n+1} \backslash E_{n}\right) \cap \operatorname{supp} f_{i(n)}\left(b_{n}\right)$, definimos $\psi_{n+1}^{*}(\xi)$ como sendo um elemento de $\mathcal{B}$ tal que

$$
\delta\left(\psi_{n+1}^{*}(\xi)\right)=\frac{r_{n+1}}{d(J) \cdot \prod_{m<n+1} d\left(f_{i(m)}\left(b_{m}\right)\right)} .
$$

Se $\xi \in E_{n} \cap \operatorname{supp} f_{i(n)}\left(b_{n}\right)$, fixemos uma raiz $d\left(f_{i(n)}\left(b_{n}\right)\right)$-ésima do ponto médio de $\psi_{n}^{*}(\xi)$. Definimos $\psi_{n+1}^{*}(\xi)$ como sendo o arco aberto de $\mathbb{T}$ centrado na raiz em questão com diâmetro

$$
\delta\left(\psi_{n+1}^{*}(\xi)\right)=\frac{r_{n+1}}{d(J) \cdot \prod_{m<n+1} d\left(f_{i(m)}\left(b_{m}\right)\right)} .
$$


Em ambos os casos, façamos

$$
\psi_{n+1}(\xi)=d(J) \cdot \prod_{m<n+1} d\left(f_{i(m)}\left(b_{m}\right)\right) \cdot \psi_{n+1}^{*}(\xi)
$$

Denotemos por $z_{\xi}$ o centro de $\psi_{n+1}^{*}(\xi)$, para todo $\xi \in \operatorname{supp} f_{i(n)}\left(b_{n}\right)$.

Seja

$$
\tilde{G}_{n+1}=\left\langle\left\{\Lambda_{(\zeta, p), c_{n+1} \cdot \prod_{m<n} d\left(g_{i(m)}\left(b_{m}\right)\right)}:(\zeta, p) \in\left(E_{n} \times e_{n}\right) \cup \operatorname{supp} g_{i(n)}\left(b_{n}\right)\right\}\right\rangle
$$

Como $\mathbb{T}$ é divisível e $G_{n}$ é um subgrupo de $\tilde{G}_{n+1}$, podemos estender $\phi \Upsilon_{G_{n}}$ a um homomorfismo de grupos $\phi \uparrow_{\tilde{G}_{n+1}}: \tilde{G}_{n+1} \rightarrow \mathbb{T}$. Fixemos $(\mu, l) \in \operatorname{supp} g_{i(n)}\left(b_{n}\right)$ tal que $q\left(g_{i(n)}\left(b_{n}\right),(\mu, l)\right) \cdot r_{n}>d(H) \cdot \prod_{m<n} d\left(g_{i(m)}\left(b_{m}\right)\right) . \quad$ Se $(\zeta, p) \in \operatorname{supp} g_{i(n)}\left(b_{n}\right) \backslash\{(\mu, l)\}$, fixemos $y_{(\zeta, p)}$ uma raiz $d\left(g_{i(n)}\left(b_{n}\right)\right)$-ésima de $\phi\left\lceil_{\tilde{G}_{n+1}}\left(\Lambda_{(\zeta, p), c_{n+1} \cdot \prod_{m<n} d\left(g_{i(m)}\left(b_{m}\right)\right)}\right)\right.$.

Aplicando o lema 4.2.3 para $P_{0} \times \omega, c_{n+1} \cdot \prod_{m<n} d\left(g_{i(m)}\left(b_{m}\right)\right), r_{n}, \psi_{n}(i(n))-u-v, g_{i(n)}\left(b_{n}\right)$, $(\mu, l)$ e $x=\phi \uparrow_{\tilde{G}_{n+1}}\left(\Lambda_{(\mu, l), c_{n+1} \cdot \prod_{m<n} d\left(g_{i(m)}\left(b_{m}\right)\right)}\right)$, onde

$$
u=\sum_{(\zeta, p) \in \operatorname{supp}} \sum_{g_{i(n)}\left(b_{n}\right) \backslash\{(\mu, l)\}} a\left(g_{i(n)}\left(b_{n}\right),(\zeta, p)\right) \cdot c_{n+1} \cdot \prod_{m<n} d\left(g_{i(m)}\left(b_{m}\right)\right) \cdot y_{(\zeta, p)}
$$

e

$$
v=\sum_{\xi \in \operatorname{supp} f_{i(n)}\left(b_{n}\right)} a\left(f_{i(n)}\left(b_{n}\right), \xi\right) \cdot d(J) \cdot \prod_{m<n} d\left(f_{i(m)}\left(b_{m}\right)\right) \cdot z_{\xi},
$$

obtemos $y_{(\mu, l)} \in \mathbb{T}$ tal que $d\left(g_{i(n)}\left(b_{n}\right)\right) \cdot y_{(\mu, l)}=\phi \uparrow_{\tilde{G}_{n+1}}\left(\Lambda_{(\mu, l), c_{n+1} \cdot \prod_{m<n} d\left(g_{i(m)}\left(b_{m}\right)\right)}\right) \mathrm{e}$

$$
a\left(g_{i(n)}\left(b_{n}\right),(\mu, l)\right) \cdot c_{n+1} \cdot \prod_{m<n} d\left(g_{i(m)}\left(b_{m}\right)\right) \cdot y_{(\mu, l)}+u+v \in \psi_{n}(i(n)) .
$$

Estendamos $\phi \uparrow_{\tilde{G}_{n+1}}$ a um homomorfismo de grupos $\phi \uparrow_{G_{n+1}}: G_{n+1} \rightarrow \mathbb{T}$ de modo que

$$
\phi\left\lceil G_{n+1}\left(\Lambda_{(\zeta, p), c_{n+1} \cdot \prod_{m<n+1} d\left(g_{i(m)}\left(b_{m}\right)\right)}\right)=y_{(\zeta, p)}\right.
$$

para todo $(\zeta, p) \in \operatorname{supp} g_{i(n)}\left(b_{n}\right)$.

Caso 5: $h_{i(n)}$ é do tipo 5 .

Uma vez que supp $g_{i(n)}\left(b_{n}\right) \backslash\left(E_{n} \times e_{n}\right) \neq \emptyset$ e o $\left(g_{i(n)}\left(b_{n}\right)\left\lceil_{\operatorname{supp} g_{i(n)}\left(b_{n}\right) \backslash\left(E_{n} \times e_{n}\right)}\right)=\mathrm{o}\left(g_{i(n)}\left(b_{n}\right)\right)\right.$, 
concluímos que

$$
\left\{g_{i(n)}\left(b_{n}\right)\right\} \cup\left\{\Lambda_{(\zeta, p), c_{n} \cdot \prod_{m<n} d\left(g_{i(m)}\left(b_{m}\right)\right)}:(\zeta, p) \in E_{n} \times e_{n}\right\}
$$

é um subconjunto independente do grupo $(\mathbb{Q} / \mathbb{Z})^{\left(\left(P_{0} \cap E\right) \times \omega\right)}$. Portanto, podemos definir $\hat{\phi}\left(g_{i(n)}\left(b_{n}\right)\right)=\sum_{(\zeta, p) \in \operatorname{supp} y_{i(n)} \cap\left(E_{n} \times e_{n}\right)} a\left(y_{i(n)},(\zeta, p)\right) \cdot c_{n-1} \cdot \prod_{m<n} d\left(g_{i(m)}\left(b_{m}\right)\right) \cdot \phi\left\lceil G_{n}\right.$ $\left(\Lambda_{(\zeta, p), c_{n} \cdot \prod_{m<n} d\left(g_{i(m)}\left(b_{m}\right)\right)}\right), \hat{\phi}(x)=\phi \Upsilon_{G_{n}}(x)$ para todo $x \in G_{n}$ e estender $\hat{\phi}$ a um homomorfismo de grupos de $\left\langle\left\{g_{i(n)}\left(b_{n}\right)\right\} \cup\left\{\Lambda_{(\zeta, p), c_{n} \cdot \prod_{m<n} d\left(g_{i(m)}\left(b_{m}\right)\right)}:(\zeta, p) \in E_{n} \times e_{n}\right\}\right\rangle \mathrm{em}$ $\mathbb{T}$. Como $\mathbb{T}$ é divisível e $\left\langle\left\{g_{i(n)}\left(b_{n}\right)\right\} \cup\left\{\Lambda_{(\zeta, p), c_{n} \cdot \prod_{m<n} d\left(g_{i(m)}\left(b_{m}\right)\right)}:(\zeta, p) \in E_{n} \times e_{n}\right\}\right\rangle$ é um subgrupo de $G_{n+1}$, podemos estender $\hat{\phi}$ a um homomorfismo de grupos $\phi\left\lceil_{G_{n+1}}: G_{n+1} \rightarrow \mathbb{T}\right.$.

Por indução, obtemos aplicações $\psi_{n}: E \cap P_{1} \rightarrow \mathcal{B}$ e $\psi_{n}^{*}: E_{n} \cap P_{1} \rightarrow \mathcal{B}$ para cada $n \in \omega$, satisfazendo as seguintes condições:

- $\overline{\psi_{n+1}(\xi)} \subset \psi_{n}(\xi)$, para todo $\xi \in E \cap P_{1}$.

- $\delta\left(\psi_{n}(\xi)\right)=r_{n}$, se $\xi \in E_{n} \cap P_{1}$ e $\psi_{n}(\xi)=\mathbb{T}$, se $\xi \in\left(E \backslash E_{n}\right) \cap P_{1}$.

- $\psi_{n}(\xi)=d(J) \cdot \prod_{m<n} d\left(f_{i(m)}\left(b_{m}\right)\right) \cdot \psi_{n}^{*}(\xi)$, se $\xi \in E_{n} \cap P_{1}$.

Obtemos, ainda, $\phi \uparrow_{G}: G \rightarrow \mathbb{T}$ um homomorfismo de grupos, onde $G=\cup_{n \in \omega} G_{n} \subset$ $(\mathbb{Q} / \mathbb{Z})^{\left(\left(P_{0} \cap E\right) \times \omega\right)}$. Usando o fato de $\mathbb{T}$ ser um grupo divisível, estendemos $\phi \uparrow_{G}$ a um

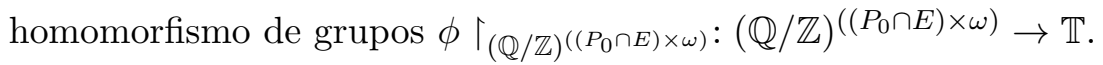

Como $\mathbb{T}$ é um espaço métrico completo e $\left(r_{n}\right)_{n \in \omega}$ é uma seqüência de números reais positivos que converge para 0 , concluímos que se $\xi \in E \cap P_{1}$, então $\cap_{n \in \omega} \psi_{n}(\xi)=\cap_{n \in \omega} \overline{\psi_{n}(\xi)}$ é um conjunto unitário. Denotaremos por $\tilde{\phi}\left(\chi_{\xi}\right)$ o único elemento de $\cap_{n \in \omega} \psi_{n}(\xi)$.

Para cada $\xi \in E \cap P_{1}$, sejam $N_{\xi}=\min \left\{n \in \omega: \xi \in E_{n}\right\}$ e $n \geq N_{\xi}$. Temos que $\psi_{n}(\xi) \neq \mathbb{T}$ e, portanto, existe um único elemento de $\psi_{n}^{*}(\xi)$ que multiplicado por $d(J) \cdot \prod_{m<n} d\left(f_{i(m)}\left(b_{m}\right)\right)$ é igual a $\tilde{\phi}\left(\chi_{\xi}\right)$. Denotaremos tal elemento por

$$
\tilde{\phi}\left(\frac{1}{d(J) \cdot \prod_{m<n} d\left(f_{i(m)}\left(b_{m}\right)\right)} \cdot \chi_{\xi}\right) .
$$

Consideremos

$$
G_{\xi}=\left\{\frac{1}{d(J) \cdot \prod_{m<n} d\left(f_{i(m)}\left(b_{m}\right)\right)} \cdot \chi_{\xi} \in \mathbb{Q}^{\left(E \cap P_{1}\right)}: n \geq N_{\xi}\right\}
$$


e denotemos por $\tilde{G}$ o grupo gerado por $\cup_{\xi \in E \cap P_{1}} G_{\xi}$. Uma vez que que se $\xi \in E \cap P_{1}$ e $n>N_{\xi}$, então

$$
\tilde{\phi}\left(\frac{1}{d(J) \cdot \prod_{m<N_{\xi}} d\left(f_{i(m)}\left(b_{m}\right)\right)} \cdot \chi_{\xi}\right)=\prod_{N_{\xi} \leq m<n} d\left(f_{i(m)}\left(b_{m}\right)\right) \cdot \tilde{\phi}\left(\frac{1}{d(J) \cdot \prod_{m<n} d\left(f_{i(m)}\left(b_{m}\right)\right)} \cdot \chi_{\xi}\right)
$$

podemos estender $\tilde{\phi}$ a um homomorfismo de grupos de $\tilde{G}$ em $\mathbb{T}$.

Usando o fato de $\mathbb{T}$ ser um grupo divisível, estendemos $\tilde{\phi}$ a um homomorfismo de grupos $\phi \Gamma_{\mathbb{Q}^{\left(P_{1} \cap E\right)}}: \mathbb{Q}^{\left(P_{1} \cap E\right)} \rightarrow \mathbb{T}$.

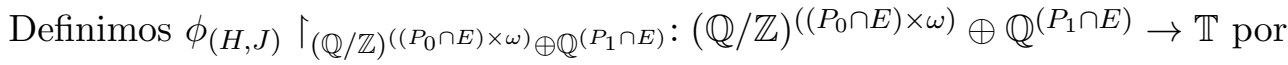

$$
\phi_{(H, J)} \uparrow_{(\mathbb{Q} / \mathbb{Z})^{\left(\left(P_{0} \cap E\right) \times \omega\right)} \oplus \mathbb{Q}^{\left(P_{1} \cap E\right)}}(\tilde{H}, \tilde{J})=\phi \Gamma_{(\mathbb{Q} / \mathbb{Z})^{\left(\left(P_{0} \cap E\right) \times \omega\right)}}(\tilde{H})+\phi \Gamma_{\mathbb{Q}^{\left(P_{1} \cap E\right)}}(\tilde{J})
$$

para todo $(\tilde{H}, \tilde{J}) \in(\mathbb{Q} / \mathbb{Z})^{\left(\left(P_{0} \cap E\right) \times \omega\right)} \oplus \mathbb{Q}^{\left(P_{1} \cap E\right)}$.

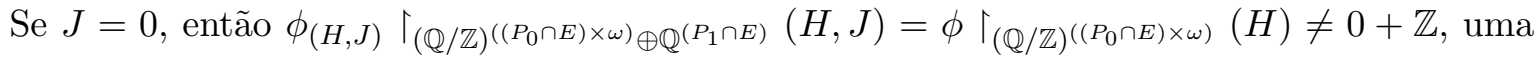
vez que $(H, J) \neq(0,0)$. Suponhamos, portanto, que $J \neq 0$. Temos que

$$
\phi \prod_{\mathbb{Q}^{\left(P_{1} \cap E\right)}}(J) \in \sum_{\xi \in \operatorname{supp} J} a(J, \xi) \cdot \psi_{0}^{*}(\xi)
$$

Como

$$
0+\mathbb{Z} \notin \phi \Gamma_{(\mathbb{Q} / \mathbb{Z})^{\left(\left(P_{0} \cap E\right) \times \omega\right)}}(H)+\sum_{\xi \in \operatorname{supp} J} a(J, \xi) \cdot \psi_{0}^{*}(\xi)
$$

temos que $\phi_{(H, J)} \Upsilon_{(\mathbb{Q} / \mathbb{Z})^{\left(\left(P_{0} \cap E\right) \times \omega\right)} \oplus \mathbb{Q}^{\left(P_{1} \cap E\right)}}(H, J) \neq 0+\mathbb{Z}$. Portanto, (i) está verificada.

Fixemos $\xi \in\left(E \cap L_{1}\right) \backslash \omega$. Para cada $k \in i^{-1}(\{\xi\})$ temos que $\phi_{(H, J)} \Upsilon_{(\mathbb{Q} / \mathbb{Z})^{\left(\left(P_{0} \cap E\right) \times \omega\right)} \oplus \mathbb{Q}^{\left(P_{1} \cap E\right)}}$ $\left(h_{i(k)}\left(b_{k}\right)\right)$ é um elemento de

$$
\phi \uparrow_{(\mathbb{Q} / \mathbb{Z})^{\left(\left(P_{0} \cap E\right) \times \omega\right)}}\left(g_{i(k)}\left(b_{k}\right)\right)+\sum_{\mu \in \operatorname{supp} f_{i(k)}\left(b_{k}\right)} a\left(f_{i(k)}\left(b_{k}\right), \mu\right) \cdot d(J) \cdot \prod_{m<k} d\left(f_{i(m)}\left(b_{m}\right)\right) \cdot \psi_{k+1}^{*}(\mu) .
$$

Além disso,

$$
\phi_{(H, J)} \Upsilon_{(\mathbb{Q} / \mathbb{Z})^{\left(\left(P_{0} \cap E\right) \times \omega\right)} \oplus \mathbb{Q}^{\left(P_{1} \cap E\right)}}\left(0, \chi_{i(k)}\right) \in \psi_{k}(i(k)) .
$$

De (4) vem que

$$
\begin{aligned}
\left.\left.\delta\left(\phi_{(H, J)}\right\rceil_{(\mathbb{Q} / \mathbb{Z})^{\left(\left(P_{0} \cap E\right) \times \omega\right)} \oplus \mathbb{Q}^{\left(P_{1} \cap E\right)}}\left(h_{i(k)}\left(b_{k}\right)\right), \phi_{(H, J)}\right\rceil_{(\mathbb{Q} / \mathbb{Z})^{\left(\left(P_{0} \cap E\right) \times \omega\right)} \oplus \mathbb{Q}^{\left(P_{1} \cap E\right)}}\left(0, \chi_{i(k)}\right)\right) & \leq d_{1}+d_{2} \\
& <2 r_{k}
\end{aligned}
$$


onde

$d_{1}=\delta\left(\phi \Gamma_{(\mathbb{Q} / \mathbb{Z})\left(\left(P_{0} \cap E\right) \times \omega\right)}\left(g_{i(k)}\left(b_{k}\right)\right)+\sum_{\mu \in \operatorname{supp} f_{i(k)}\left(b_{k}\right)} a\left(f_{i(k)}\left(b_{k}\right), \mu\right) \cdot d(J) \cdot \prod_{m<k} d\left(f_{i(m)}\left(b_{m}\right)\right) \cdot \psi_{k+1}^{*}(\mu)\right)$

e

$$
d_{2}=\delta\left(\psi_{k}(i(k))\right)
$$

Como $r_{k} \rightarrow 0$, concluímos que a seqüência $\left\{\phi_{(H, J)} \uparrow_{(\mathbb{Q} / \mathbb{Z})^{\left(\left(P_{0} \cap E\right) \times \omega\right)} \oplus \mathbb{Q}^{\left(P_{1} \cap E\right)}}\left(h_{i(k)}\left(b_{k}\right)\right): k \in\right.$ $\left.i^{-1}(\{\xi\})\right\}$ converge para $\phi_{(H, J)} \Upsilon_{(\mathbb{Q} / \mathbb{Z})^{\left(\left(P_{0} \cap E\right) \times \omega\right)} \oplus \mathbb{Q}^{\left(P_{1} \cap E\right)}}\left(0, \chi_{\xi}\right)$. Da propriedade (v) do lema 5.3.2 segue que

$\phi_{(H, J)} \Upsilon_{(\mathbb{Q} / \mathbb{Z})^{\left(\left(P_{0} \cap E\right) \times \omega\right)} \oplus \mathbb{Q}^{\left(P_{1} \cap E\right)}}\left(0, \chi_{\xi}\right)=p_{\xi}-\lim \left\{\phi_{(H, J)}\left\lceil_{(\mathbb{Q} / \mathbb{Z})^{\left(\left(P_{0} \cap E\right) \times \omega\right)} \oplus \mathbb{Q}^{\left(P_{1} \cap E\right)}}\left(h_{\xi}(n)\right): n \in \omega\right\}\right.$.

Logo, (ii) está verificada.

Por fim, seja $\xi \in E \cap \bigcup_{n \in D} L_{n}$. Como $E \times \omega=\cup_{n \in \omega}\left(E_{n} \times e_{n}\right)$, existe $n \in \omega$ tal que supp $y_{\xi} \subset E_{n} \times e_{n}$. Para cada $k \in i^{-1}(\{\xi\})$ temos que $\phi_{(H, J)} \Upsilon_{(\mathbb{Q} / \mathbb{Z})\left(\left(P_{0} \cap E\right) \times \omega\right) \oplus \mathbb{Q}^{\left(P_{1} \cap E\right)}}$ $\left(h_{i(k)}\left(b_{k}\right)\right)=\phi \uparrow_{(\mathbb{Q} / \mathbb{Z})^{\left(\left(P_{0} \cap E\right) \times \omega\right)}}\left(g_{i(k)}\left(b_{k}\right)\right)$, uma vez que $h_{i(k)}$ é do tipo 5 . Além disso, temos que $\phi \uparrow_{(\mathbb{Q} / \mathbb{Z})^{\left(\left(P_{0} \cap E\right) \times \omega\right)}}\left(g_{i(k)}\left(b_{k}\right)\right)=\phi \uparrow_{(\mathbb{Q} / \mathbb{Z})_{\left(\left(P_{0} \cap E\right) \times \omega\right)}}\left(y_{i(k)}\right)$, para todo $k>n$. Da propriedade $(\mathrm{v})$ do lema 5.3.2 segue que

$\phi_{(H, J)} \Upsilon_{(\mathbb{Q} / \mathbb{Z})^{\left(\left(P_{0} \cap E\right) \times \omega\right)} \oplus \mathbb{Q}^{\left(P_{1} \cap E\right)}}\left(y_{\xi}, 0\right)=p_{\xi}-\lim \left\{\phi_{(H, J)} \Upsilon_{(\mathbb{Q} / \mathbb{Z})^{\left(\left(P_{0} \cap E\right) \times \omega\right)} \oplus \mathbb{Q}^{\left(P_{1} \cap E\right)}}\left(h_{\xi}(n)\right): n \in \omega\right\}$.

Logo, (iii) está verificada.

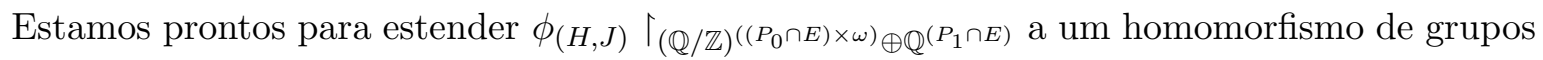
$\phi_{(H, J)}:(\mathbb{Q} / \mathbb{Z})^{\left(P_{0} \times \omega\right)} \oplus \mathbb{Q}^{\left(P_{1}\right)} \rightarrow \mathbb{T}$

Lema 5.3.4. Para cada $(H, J) \in(\mathbb{Q} / \mathbb{Z})^{\left(P_{0} \times \omega\right)} \oplus \mathbb{Q}^{\left(P_{1}\right)} \backslash\{(0,0)\}$, existe $\phi_{(H, J)}:(\mathbb{Q} / \mathbb{Z})^{\left(P_{0} \times \omega\right)} \oplus$ $\mathbb{Q}^{\left(P_{1}\right)} \rightarrow \mathbb{T}$ um homomorfismo de grupos tal que:

(i) $\phi_{(H, J)}(H, J) \neq 0+\mathbb{Z}$;

(ii) $\phi_{(H, J)}\left(0, \chi_{\xi}\right)=p_{\xi}-\lim \left\{\phi_{(H, J)}\left(h_{\xi}(n)\right): n \in \omega\right\}$, para cada $\xi \in L_{1} \backslash \omega$.

(iii) $\phi_{(H, J)}\left(y_{\xi}, 0\right)=p_{\xi}-\lim \left\{\phi_{(H, J)}\left(h_{\xi}(n)\right): n \in \omega\right\}$, para cada $\xi \in \cup_{n \in D} L_{n}$.

Demonstração. Faremos a construção de $\phi_{(H, J)}$ por indução. De acordo com a proposição 5.3.1, existe $E \in[\mathfrak{c}]^{\omega}$ tal que $\operatorname{supp}(H, J) \subset(E \times \omega) \cup E,\left|E \cap\left[\left(L_{1} \cup \bigcup_{n \in D} L_{n}\right) \backslash \omega\right]\right|=\omega$ e 
$\cup_{n \in \omega} \operatorname{supp} h_{\xi}(n) \subset(E \times \omega) \cup E$, qualquer que seja $\xi \in E \cap\left[\left(L_{1} \cup \cup_{n \in D} L_{n}\right) \backslash \omega\right]$. Segundo o

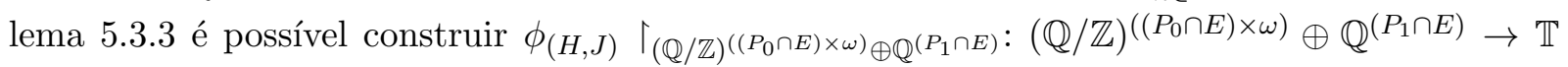
um homomorfismo de grupos tal que:

(1) $\phi_{(H, J)} \Upsilon_{(\mathbb{Q} / \mathbb{Z})^{\left(\left(P_{0} \cap E\right) \times \omega\right)} \oplus \mathbb{Q}^{\left(P_{1} \cap E\right)}}(H, J) \neq 0+\mathbb{Z}$;

(2) $\phi_{(H, J)} \Upsilon_{(\mathbb{Q} / \mathbb{Z})^{\left(\left(P_{0} \cap E\right) \times \omega\right)} \oplus \mathbb{Q}^{\left(P_{1} \cap E\right)}}\left(0, \chi_{\xi}\right)=p_{\xi}-\lim \left\{\phi_{(H, J)} \Upsilon_{(\mathbb{Q} / \mathbb{Z})^{\left(\left(P_{0} \cap E\right) \times \omega\right)} \oplus \mathbb{Q}^{\left(P_{1} \cap E\right)}}\left(h_{\xi}(n)\right): n \in\right.$ $\omega\}$, para cada $\xi \in\left(E \cap L_{1}\right) \backslash \omega$;

(3) $\phi_{(H, J)} \Upsilon_{(\mathbb{Q} / \mathbb{Z})^{\left(\left(P_{0} \cap E\right) \times \omega\right)} \oplus \mathbb{Q}^{\left(P_{1} \cap E\right)}}\left(y_{\xi}, 0\right)=p_{\xi}-\lim \left\{\phi_{(H, J)} \Upsilon_{(\mathbb{Q} / \mathbb{Z})^{\left(\left(P_{0} \cap E\right) \times \omega\right)} \oplus \mathbb{Q}^{\left(P_{1} \cap E\right)}}\left(h_{\xi}(n)\right): n \in\right.$ $\omega\}$, para cada $\xi \in E \cap \cup_{n \in D} L_{n}$.

Consideremos $\left\{\alpha_{\xi}: \xi<\mathfrak{c}\right\}$ uma indexação estritamente crescente de $\mathfrak{c} \backslash E$.

- Se $\alpha_{0} \in P_{0} \backslash \bigcup_{n \in D} L_{n}$, usamos o fato de $\mathbb{T}$ ser um grupo divisível para estender $\phi_{(H, J)} \uparrow_{(\mathbb{Q} / \mathbb{Z})}\left(\left(P_{0} \cap E\right) \times \omega\right) \oplus \mathbb{Q}^{\left(P_{1} \cap E\right)}$ a um homomorfismo de grupos

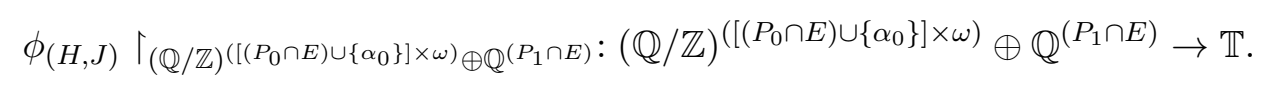

- Se $\alpha_{0} \in P_{1} \backslash\left(L_{1} \backslash \omega\right)$, usamos o fato de $\mathbb{T}$ ser um grupo divisível para estender $\phi_{(H, J)} \uparrow_{(\mathbb{Q} / \mathbb{Z})^{\left(\left(P_{0} \cap E\right) \times \omega\right)} \oplus \mathbb{Q}^{\left(P_{1} \cap E\right)}}$ a um homomorfismo de grupos

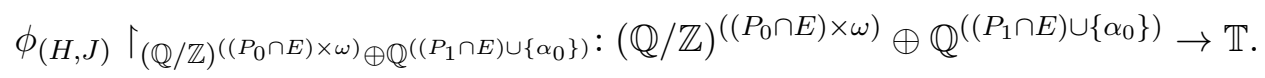

- Se $\alpha_{0} \in \cup_{n \in D} L_{n}$, definamos

$$
\tilde{\phi}_{(H, J)}\left(y_{\alpha_{0}}, 0\right)=p_{\alpha_{0}}-\lim \left\{\phi_{(H, J)} \Upsilon_{(\mathbb{Q} / \mathbb{Z})^{\left(\left(P_{0} \cap E\right) \times \omega\right)} \oplus \mathbb{Q}^{\left(P_{1} \cap E\right)}}\left(h_{\alpha_{0}}(n)\right): n \in \omega\right\}
$$

e façamos

$$
\tilde{\phi}_{(H, J)}(\tilde{H}, \tilde{J})=\phi_{(H, J)} \Upsilon_{(\mathbb{Q} / \mathbb{Z})}{ }_{\left(\left(P_{0} \cap E\right) \times \omega\right) \oplus \mathbb{Q}^{\left(P_{1} \cap E\right)}}(\tilde{H}, \tilde{J})
$$

para todo $(\tilde{H}, \tilde{J}) \in(\mathbb{Q} / \mathbb{Z})^{\left(\left(P_{0} \cap E\right) \times \omega\right)} \oplus \mathbb{Q}^{\left(P_{1} \cap E\right)}$.

Seja $G_{0}$ o subgrupo de $(\mathbb{Q} / \mathbb{Z})^{\left(\left[\left(P_{0} \cap E\right) \cup\left\{\alpha_{0}\right\}\right] \times \omega\right)}$ gerado por $(\mathbb{Q} / \mathbb{Z})^{\left(\left(P_{0} \cap E\right) \times \omega\right)} \cup\left\{y_{\alpha_{0}}\right\}$. Podemos estender $\tilde{\phi}_{(H, J)}$ a um homomorfismo de grupos de $G_{0} \oplus \mathbb{Q}^{\left(P_{1} \cap E\right)}$ em $\mathbb{T}$. Uma vez que $\mathbb{T}$ é divisível, estendemos $\tilde{\phi}_{(H, J)}$ a um homomorfismo de grupos

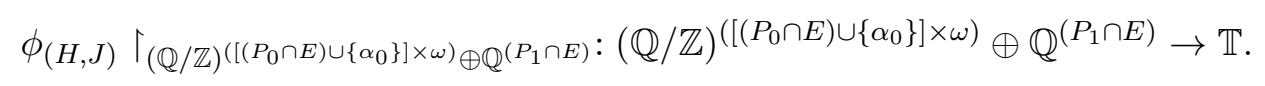


- Se $\alpha_{0} \in L_{1} \backslash \omega$, definamos

$$
\tilde{\phi}_{(H, J)}\left(0, \chi_{\alpha_{0}}\right)=p_{\alpha_{0}}-\lim \left\{\phi_{(H, J)} \Upsilon_{(\mathbb{Q} / \mathbb{Z})^{\left(\left(P_{0} \cap E\right) \times \omega\right)} \oplus \mathbb{Q}^{\left(P_{1} \cap E\right)}}\left(h_{\alpha_{0}}(n)\right): n \in \omega\right\}
$$

e façamos

$$
\tilde{\phi}_{(H, J)}(\tilde{H}, \tilde{J})=\phi_{(H, J)} \uparrow_{(\mathbb{Q} / \mathbb{Z})^{\left(\left(P_{0} \cap E\right) \times \omega\right)} \oplus \mathbb{Q}^{\left(P_{1} \cap E\right)}}(\tilde{H}, \tilde{J})
$$

para todo $(\tilde{H}, \tilde{J}) \in(\mathbb{Q} / \mathbb{Z})^{\left(\left(P_{0} \cap E\right) \times \omega\right)} \oplus \mathbb{Q}^{\left(P_{1} \cap E\right)}$.

Seja $G_{0}$ o subgrupo de $\mathbb{Q}^{\left(\left(P_{1} \cap E\right) \cup\left\{\alpha_{0}\right\}\right)}$ gerado por $\mathbb{Q}^{\left(P_{1} \cap E\right)} \cup\left\{\chi_{\alpha_{0}}\right\}$. Podemos estender $\tilde{\phi}_{(H, J)}$ a um homomorfismo de grupos de $(\mathbb{Q} / \mathbb{Z})^{\left(\left(P_{0} \cap E\right) \times \omega\right)} \oplus G_{0}$ em $\mathbb{T}$. Uma vez que $\mathbb{T}$ é divisível, estendemos $\tilde{\phi}_{(H, J)}$ a um homomorfismo de grupos

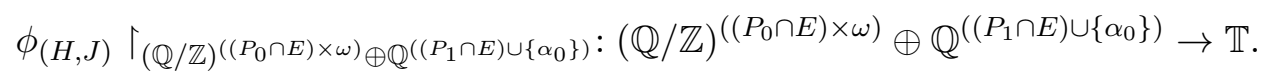

Repetindo indutivamente esta construção, obteremos $\phi_{(H, J)}:(\mathbb{Q} / \mathbb{Z})^{\left(P_{0} \times \omega\right)} \oplus \mathbb{Q}^{\left(P_{1}\right)} \rightarrow \mathbb{T}$ um homomorfismo de grupos satisfazendo (i), (ii) e (iii).

\subsection{Explicitando a topologia}

A próxima proposição mostra que o produto diagonal da família $\left\{\phi_{(H, J)}:(H, J) \in\right.$ $\left.(\mathbb{Q} / \mathbb{Z})^{\left(P_{0} \times \omega\right)} \oplus \mathbb{Q}^{\left(P_{1}\right)} \backslash\{(0,0)\}\right\}$ é um monomorfismo de grupos.

Proposição 5.4.1. Suponhamos que para cada $(H, J) \in(\mathbb{Q} / \mathbb{Z})^{\left(P_{0} \times \omega\right)} \oplus \mathbb{Q}^{\left(P_{1}\right)} \backslash\{(0,0)\}$ exista um homomorfismo de grupos $\phi_{(H, J)}:(\mathbb{Q} / \mathbb{Z})^{\left(P_{0} \times \omega\right)} \oplus \mathbb{Q}^{\left(P_{1}\right)} \rightarrow \mathbb{T}$ tal que $\phi_{(H, J)}(H, J) \neq 0+\mathbb{Z}$. A aplicação

$$
\begin{array}{cccc}
\Phi:(\mathbb{Q} / \mathbb{Z})^{\left(P_{0} \times \omega\right)} \oplus \mathbb{Q}^{\left(P_{1}\right)} & \rightarrow & \mathbb{T}^{(\mathbb{Q} / \mathbb{Z})^{\left(P_{0} \times \omega\right)} \oplus \mathbb{Q}^{\left(P_{1}\right)} \backslash\{(0,0)\}} \\
(\tilde{H}, \tilde{J}) & \mapsto & \Phi(\tilde{H}, \tilde{J})
\end{array}
$$

dada por

$$
\Phi(\tilde{H}, \tilde{J})(H, J)=\phi_{(H, J)}(\tilde{H}, \tilde{J}), \text { para cada }(H, J) \in(\mathbb{Q} / \mathbb{Z})^{\left(P_{0} \times \omega\right)} \oplus \mathbb{Q}^{\left(P_{1}\right)} \backslash\{(0,0)\}
$$

é um monomorfismo de grupos.

Demonstração. Análoga à da proposição 2.3.1. 
Teorema 5.4.2. Se $G$ é um grupo abeliano de não torção tal que $|G|=\mathfrak{c},|G / T(G)|=\mathfrak{c}$ e, para quaisquer $n, d \in \mathbb{N} \backslash\{0\}$ tais que $d \mid n$, o conjunto $d G[n]$ é finito ou tem cardinalidade $\mathfrak{c}$, então $G$ admite uma topologia que o torna um grupo topológico enumeravelmente compacto sem seqüências não triviais convergentes.

Demonstração. Seja

$$
\tau=\left\{\varphi^{-1} \circ \Phi^{-1}(U \cap \Phi[\varphi[G]]): U \text { é aberto em } \mathbb{T}^{(\mathbb{Q} / \mathbb{Z})^{\left(P_{0} \times \omega\right)} \oplus \mathbb{Q}^{\left(P_{1}\right)} \backslash\{(0,0)\}}\right\}
$$

Temos que $\tau$ é uma topologia em $G$ que o torna um grupo topológico.

Consideremos $h: \omega \rightarrow G$. Se $h$ é trivial, nada temos a fazer. Se não, $h$ possui duas subseqüências constantes e distintas ou existe $j: \omega \rightarrow \omega$ estritamente crescente tal que $h \circ j$ é injetora. No primeiro caso, temos que $\{h(n) \in G: n \in \omega\}$ tem pelo menos dois pontos de acumulação distintos e, portanto, $h$ não é convergente. Suponhamos que o segundo caso ocorra.

As aplicações

$$
\begin{aligned}
h_{0}: \omega & \rightarrow G \\
n & \mapsto(h \circ j)(2 n)
\end{aligned}
$$

e

$$
\begin{aligned}
h_{1}: \omega & \rightarrow G \\
n & \mapsto(h \circ j)(2 n+1)
\end{aligned}
$$

são subseqüências distintas de $h$. De acordo com a proposição 5.2.4, existem $j_{0}, j_{1}: \omega \rightarrow \omega$ estritamente crescentes e $c_{0}, c_{1} \in \varphi[G]$ tais que $\tilde{h}_{0}, \tilde{h}_{1} \in \mathcal{H}$, onde $\tilde{h}_{0}, \tilde{h}_{1}: \omega \rightarrow \varphi[G]$ são dadas por $\tilde{h}_{0}(n)=\varphi \circ h_{0} \circ j_{0}(n)+c_{0}$ e $\tilde{h}_{1}(n)=\varphi \circ h_{1} \circ j_{1}(n)+c_{1}$, para todo $n \in \omega$. Logo, existem $\xi_{0}, \xi_{1} \in\left(L_{1} \cup \bigcup_{n \in D} L_{n}\right) \backslash \omega$ distintos tais que $\tilde{h}_{0}=h_{\xi_{0}}$ e $\tilde{h}_{1}=h_{\xi_{1}}$.

Seja $i \in\{0,1\}$. Se $h_{\xi_{i}}$ é do tipo $1,2,3$ ou 4 , então

$$
\phi_{(H, J)}\left(0, \chi_{\xi_{i}}\right)=p_{\xi_{i}}-\lim \left\{\phi_{(H, J)}\left(h_{\xi_{i}}(n)\right): n \in \omega\right\}
$$

para todo $(H, J) \in \backslash(\mathbb{Q} / \mathbb{Z})^{\left(P_{0} \times \omega\right)} \oplus \mathbb{Q}^{\left(P_{1}\right)}\{(0,0)\}$ e, portanto,

$$
\Phi\left(0, \chi_{\xi_{i}}\right)=p_{\xi_{i}}-\lim \left\{\Phi\left(h_{\xi_{i}}(n)\right): n \in \omega\right\}
$$

Logo,

$$
\varphi^{-1}\left(0, \chi_{\xi_{i}}\right)-\varphi^{-1}\left(c_{i}\right)=p_{\xi_{i}}-\lim \left\{\left(h_{i} \circ j_{i}\right)(n): n \in \omega\right\}
$$


Se $h_{\xi_{i}}$ é do tipo 5 , então

$$
\phi_{(H, J)}\left(y_{\xi_{i}}, 0\right)=p_{\xi_{i}}-\lim \left\{\phi_{(H, J)}\left(h_{\xi_{i}}(n)\right): n \in \omega\right\}
$$

para todo $(H, J) \in \backslash(\mathbb{Q} / \mathbb{Z})^{\left(P_{0} \times \omega\right)} \oplus \mathbb{Q}^{\left(P_{1}\right)}\{(0,0)\}$ e, portanto,

$$
\Phi\left(y_{\xi_{i}}, 0\right)=p_{\xi_{i}}-\lim \left\{\Phi\left(h_{\xi_{i}}(n)\right): n \in \omega\right\}
$$

Logo,

$$
\varphi^{-1}\left(y_{\xi_{i}}, 0\right)-\varphi^{-1}\left(c_{i}\right)=p_{\xi_{i}}-\lim \left\{\left(h_{i} \circ j_{i}\right)(n): n \in \omega\right\} .
$$

Assim, $\{h(n) \in G: n \in \omega\}$ tem dois pontos de acumulação distintos.

\subsection{Caracterização algébrica dos grupos abelianos de não torção que têm cardinalidade $\mathfrak{c}$ e que admitem uma topologia de grupo enumeravelmente compacta}

Proposição 5.5.1. Se $G$ é um grupo topológico pseudocompacto infinito, então $|G| \geq \mathfrak{c}$.

Demonstração. Ver teorema 9.11.2 de [1].

Proposição 5.5.2. Seja $G$ um grupo abeliano de não torção. Se $G$ admite uma topologia de grupo pseudocompacta, então $|G / T(G)| \geq \mathfrak{c}$.

Demonstração. Ver teorema 9.11 .5 de [1].

Como todo espaço topológico enumeravelmente compacto é, em particular, pseudocompacto, obtemos os seguintes corolários:

Corolário 5.5.3. Seja $G$ um grupo abeliano de não torção tal que $|G|=\mathfrak{c}$. Se $G$ admite uma topologia de grupo enumeravelmente compacta então, para cada número natural $n>1$ e para cada divisor d de $n$, o conjunto $d G[n]$ é finito ou tem cardinalidade $\mathfrak{c}$.

Demonstração. Seja $\tau$ uma topologia enumeravelmente compacta em $G$ que o torna um grupo topológico. A aplicação $\varphi_{n}: G \rightarrow G$ dada por $\varphi_{n}(x)=n x$ para cada $x \in G$, é contínua. Logo, $G[n]=\varphi_{n}^{-1}\left(\left\{0_{G}\right\}\right)$ é um subgrupo fechado de $G$ e, portanto, enumeravelmente compacto. Como $\varphi_{d}$ é um homomorfismo de grupos contínuo, temos que $d G[n]=\varphi_{d}[G[n]]$ é um subgrupo 
enumeravelmente compacto de $G[n]$. Da proposição 5.5.1 segue que $d G[n]$ é finito ou tem cardinalidade $\mathfrak{c}$.

Corolário 5.5.4. Seja $G$ um grupo abeliano de não torção tal que $|G|=\mathfrak{c}$. Se $G$ admite uma topologia de grupo enumeravelmente compacta, então $|G / T(G)|=\mathfrak{c}$.

Demonstração. Decorre imediatamente da proposição 5.5.2.

Teorema 5.5.5. Seja $G$ um grupo abeliano de não torção tal que $|G|=\mathfrak{c}$. As seguintes condições são equivalentes:

(i) G admite uma topologia de grupo enumeravelmente compacta;

(ii) G admite uma topologia de grupo enumeravelmente compacta sem seqüências não triviais convergentes;

(iii) $|G / T(G)|=\mathfrak{c}$ e para quaisquer $n, d \in \mathbb{N} \backslash\{0\}$ com $d \mid n$, o conjunto $d G[n]$ é finito ou tem cardinalidade $\mathfrak{c}$.

Demonstração. Claramente, (ii) implica (i). Dos corolários 5.5.3 e 5.5.4 decorre que (i) implica (iii). Por fim, do teorema 5.4.2 segue que (iii) implica (ii). ${ }^{11}$

\subsection{Aumentando o peso do grupo}

Teorema 5.6.1. Seja $G$ um grupo abeliano de não torção tal que $|G|=\mathfrak{c},|G / T(G)|=\mathfrak{c}$ e, para quaisquer $n, d \in \mathbb{N} \backslash\{0\}$ com $d \mid n$, o conjunto $d G[n]$ é finito ou tem cardinalidade $\mathfrak{c}$. Existe uma topologia em $G$ que o torna um grupo topológico enumeravelmente compacto sem seqüências não triviais convergentes com peso $2^{\mathfrak{c}}$.

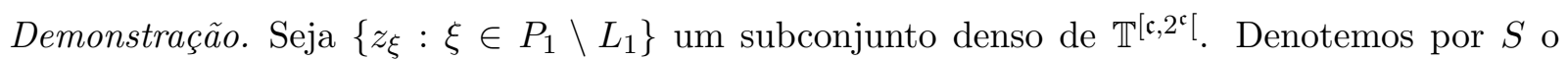
subgrupo de $(\mathbb{Q} / \mathbb{Z})^{\left(P_{0} \times \omega\right)} \oplus \mathbb{Q}^{\left(P_{1}\right)}$ gerado por $\left\{\left(0, \chi_{\xi}\right) \in(\mathbb{Q} / \mathbb{Z})^{\left(P_{0} \times \omega\right)} \oplus \mathbb{Q}^{\left(P_{1}\right)}: \xi \in P_{1} \backslash L_{1}\right\}$

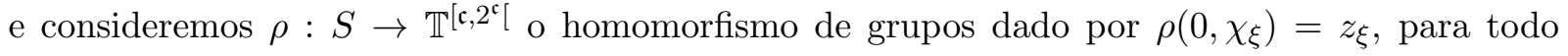
$\xi \in P_{1} \backslash L_{1}$. Como $\mathbb{T}^{\left[\mathfrak{c}, 2^{\mathfrak{c}}[\right.}$ é divisível, podemos estender $\rho$ a um homomorfismo de grupos $\rho:(\mathbb{Q} / \mathbb{Z})^{\left(\left(P_{0} \backslash \cup_{n \in D} L_{n}\right) \times \omega\right)} \oplus Q^{\left(\left(P_{1} \backslash L_{1}\right) \cup \omega\right)} \rightarrow \mathbb{T}^{\left[\mathfrak{c}, 2^{\mathfrak{c}}[\right.}$.

\footnotetext{
${ }^{11}$ As implicações (ii) $\Rightarrow$ (i) e (i) $\Rightarrow$ (iii) valem em ZFC. Usamos a existência de ultrafiltros seletivos apenas para mostrar que (iii) $\Rightarrow$ (ii).
} 
Seja $\left\{\alpha_{\xi}: \xi<\mathfrak{c}\right\}$ uma indexação estritamente crescente de $\left(L_{1} \cup \bigcup_{n \in D} L_{n}\right) \backslash \omega$. Definimos

$$
z_{\alpha_{0}}=p_{\alpha_{0}}-\lim \left\{\rho\left(h_{\alpha_{0}}(n)\right): n \in \omega\right\}
$$

e façamos $\rho\left(0, \chi_{\alpha_{0}}\right)=z_{\alpha_{0}}$, se $\alpha_{0} \in L_{1} \backslash \omega$ e $\rho\left(y_{\alpha_{0}}, 0\right)=z_{\alpha_{0}}$, se $\alpha_{0} \in \cup_{n \in D} L_{n}$.

Repetindo indutivamente esta construção, obtemos $\rho:(\mathbb{Q} / \mathbb{Z})^{\left(P_{0} \times \omega\right)} \oplus \mathbb{Q}^{\left(P_{1}\right)} \rightarrow \mathbb{T}^{\left[\mathfrak{c}, 2^{\mathfrak{c}}[\right.}$ um homomorfismo de grupos tal que $\rho\left(0, \chi_{\xi}\right)=p_{\xi}-\lim \left\{\rho\left(h_{\xi}(n)\right): n \in \omega\right\}$, se $\xi \in L_{1} \backslash \omega$ e $\rho\left(y_{\xi}, 0\right)=p_{\xi}-\lim \left\{\rho\left(h_{\xi}(n)\right): n \in \omega\right\}$, se $\xi \in \cup_{n \in D} L_{n}$.

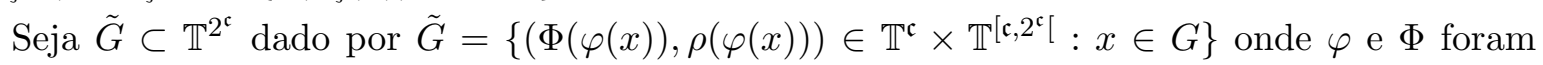
definidas nas proposições 5.1.4 e 5.4.1, respectivamente. Temos que $G$ é isomorfo a $\tilde{G}$ e $\tilde{G}$, munido da topologia de subespaço induzida por $\mathbb{T}^{2^{\mathfrak{c}}}$ é um grupo topológico enumeravelmente compacto sem seqüências não triviais convergentes com peso $2^{\mathfrak{c}}$. 
CAPÍTULO 6

\section{Sobre o número de topologias de grupo enumeravelmente compactas que um grupo abeliano de não torção e cardinalidade $\mathfrak{c}$ admite}

Neste capítulo, assumiremos a existência de $2^{\mathfrak{c}}$ ultrafiltros seletivos para mostrar que se um grupo abeliano de não torção e cardinalidade $\mathfrak{c}$ admite uma topologia de grupo enumeravelmente compacta, então o mesmo admite $2^{\mathfrak{c}}$ topologias enumeravelmente compactas (duas a duas não homeomorfas) que o tornam um grupo topológico.

Começaremos definindo o invariante topológico $\mathcal{F}$ e, após uma breve recordação da notação adotada no capítulo 5 , mostraremos que se $G$ é um grupo abeliano de não torção e cardinalidade $\mathfrak{c}$ que admite uma topologia de grupo enumeravelmente compacta, então dados $\kappa<2^{\mathfrak{c}}$ um cardinal e $\left\{\left(X_{\alpha}, \tau_{\alpha}\right): \alpha<\kappa\right\}$ uma família de espaços topológicos, é possível munir $G$ de uma topologia $\tau$ que o torna um grupo topológico enumeravelmente compacto com $\mathcal{F}(G, \tau) \neq \mathcal{F}\left(X_{\alpha}, \tau_{\alpha}\right)$ para todo $\alpha<\kappa$.

\subsection{O invariante topológico $\mathcal{F}$}

Recordamos que se $(X, \tau)$ é um espaço topológico e $x \in X$, então $\tau_{x}$ denota o conjunto de todas as vizinhanças abertas de $x$ em $X$.

Definição 6.1.1. Se $X$ é um espaço topológico e $x \in X$ é um ponto de acumulação de 
$\left\{x_{n}: n \in \omega\right\} \subset X$, definimos

$$
\mathcal{F}\left(X,\left\{x_{n}: n \in \omega\right\}, x\right)=\left\{A \subset \omega: \exists U \in \tau_{x} \text { tal que }\left\{n \in \omega: x_{n} \in U\right\} \subset A\right\} .
$$

Proposição 6.1.2. Se $X$ é um espaço topológico e $x \in X$ é um ponto de acumulação de $\left\{x_{n}: n \in \omega\right\} \subset X$, então $\mathcal{F}\left(X,\left\{x_{n}: n \in \omega\right\}, x\right)$ é um filtro sobre $\omega$.

Demonstração. Como $x$ é ponto de acumulação de $\left\{x_{n}: n \in \omega\right\}$, para cada $U \in \tau_{x}$ existe $n \in \omega$ tal que $x_{n} \in U$. Logo, $\emptyset \notin \mathcal{F}\left(X,\left\{x_{n}: n \in \omega\right\}, x\right)$ e $\omega \in \mathcal{F}\left(X,\left\{x_{n}: n \in \omega\right\}, x\right)$. Se $A, B \in \mathcal{F}\left(X,\left\{x_{n}: n \in \omega\right\}, x\right)$, então existem $U, V \in \tau_{x}$ tais que $\left\{n \in \omega: x_{n} \in U\right\} \subset A$ e $\left\{n \in \omega: x_{n} \in V\right\} \subset B$. Como $U \cap V \in \tau_{x}$ e $\left\{n \in \omega: x_{n} \in U \cap V\right\} \subset A \cap B$, temos que $A \cap B \in \mathcal{F}\left(X,\left\{x_{n}: n \in \omega\right\}, x\right)$. Por fim, se $A \in \mathcal{F}\left(X,\left\{x_{n}: n \in \omega\right\}, x\right)$ e $B \subset \omega$ são tais que $A \subset B$, então existe $U \in \tau_{x}$ tal que $\left\{n \in \omega: x_{n} \in U\right\} \subset A \subset B$. Logo, $B \in \mathcal{F}\left(X,\left\{x_{n}: n \in \omega\right\}, x\right)$. Portanto, $\mathcal{F}\left(X,\left\{x_{n}: n \in \omega\right\}, x\right)$ é um filtro sobre $\omega$.

Denotaremos por $\mathcal{F}(X)$ a família de todos os filtros $\mathcal{F}\left(X,\left\{x_{n}: n \in \omega\right\}, x\right)$, onde $X$ é um espaço topológico fixado e $x \in X$ é um ponto de acumulação de $\left\{x_{n}: n \in \omega\right\} \subset X$. Observamos que se $|X|=\mathfrak{c}$, então $|\mathcal{F}(X)| \leq \mathfrak{c}^{1}$

Lema 6.1.3. Sejam $X$ e $Y$ espaços topológicos e $h: X \rightarrow Y$ um homeomorfismo. Se $x \in X$ é um ponto de acumulação de $\left\{x_{n}: n \in \omega\right\} \subset X$, então $h(x) \in Y$ é um ponto de acumulação de $\left\{h\left(x_{n}\right): n \in \omega\right\} \subset Y$ e $\mathcal{F}\left(X,\left\{x_{n}: n \in \omega\right\}, x\right)=\mathcal{F}\left(Y,\left\{h\left(x_{n}\right): n \in \omega\right\}, h(x)\right)$.

Demonstração. Seja $U$ uma vizinhança aberta de $h(x)$ em $Y$. Como $h$ é contínua, temos que $h^{-1}(U)$ é uma vizinhança aberta de $x$ em $X$. Do fato de $x$ ser ponto de acumulação de $\left\{x_{n}: n \in \omega\right\}$ decorre que $\left(\left\{x_{n}: n \in \omega\right\} \backslash\{x\}\right) \cap h^{-1}(U) \neq \emptyset$. Logo, existe $n \in \omega$ tal que $x_{n} \in h^{-1}(U) \backslash\{x\}$ e, portanto, $h\left(x_{n}\right) \in U \backslash\{h(x)\}$ uma vez que $h$ é injetora. Com isso, concluímos que $\left(\left\{h\left(x_{n}\right): n \in \omega\right\} \backslash\{h(x)\}\right) \cap U \neq \emptyset$. Logo, $h(x)$ é um ponto de acumulação de $\left\{h\left(x_{n}\right): n \in \omega\right\}$.

Se $A \in \mathcal{F}\left(X,\left\{x_{n}: n \in \omega\right\}, x\right)$, então existe $U$ uma vizinhança aberta de $x$ em $X$ tal que $\left\{n \in \omega: x_{n} \in U\right\} \subset A$. Como $h$ é um homeomorfismo, temos que $h(U)$ é uma vizinhança aberta de $h(x)$ em $Y$ e $\left\{n \in \omega: h\left(x_{n}\right) \in h(U)\right\}=\left\{n \in \omega: x_{n} \in U\right\} \subset A$. Portanto, $A \in \mathcal{F}\left(Y,\left\{h\left(x_{n}\right): n \in \omega\right\}, h(x)\right)$. Analogamente, mostramos que $\mathcal{F}\left(Y,\left\{h\left(x_{n}\right): n \in \omega\right\}, h(x)\right) \subset$ $\mathcal{F}\left(X,\left\{x_{n}: n \in \omega\right\}, x\right)$.

Corolário 6.1.4. Se $X$ e $Y$ são espaços topológicos homeomorfos, então $\mathcal{F}(X)=\mathcal{F}(Y)$.

\footnotetext{
${ }^{1}$ Basta notar que $\left|[\mathfrak{c}]^{\leq \omega}\right|=\mathfrak{c}$ e que $\mathfrak{c} \times \mathfrak{c}$ é equipotente a $\mathfrak{c}$.
} 


\subsection{Recordando a notação adotada no capítulo 5}

Seja $G$ um grupo abeliano de não torção e cardinalidade $\mathfrak{c}$ que admite uma topologia de grupo enumeravelmente compacta. ${ }^{2}$ Denotaremos por $D$ o conjunto dos números naturais $n>1$ tais que $G$ contém uma cópia de $\mathbb{Z}_{n}^{(\mathfrak{c})}$ e consideraremos $\left\{P_{0}, P_{1}\right\}$ uma partição de $\mathfrak{c}$ tal que $\left|P_{0}\right|=\left|P_{1}\right|=\mathfrak{c}, \omega \cup\{\omega\} \subset P_{1}$ e $\{\omega+n: n \geq 1\} \subset P_{0}$. Tomemos $\varphi: G \rightarrow(\mathbb{Q} / \mathbb{Z})^{\left(P_{0} \times \omega\right)} \oplus \mathbb{Q}^{\left(P_{1}\right)}$ um monomorfismo de grupos tal que

$$
\left\{\left(0, \chi_{\xi}\right) \in(\mathbb{Q} / \mathbb{Z})^{\left(P_{0} \times \omega\right)} \oplus \mathbb{Q}^{\left(P_{1}\right)}: \xi \in L_{1}\right\} \subset \varphi[G]
$$

e

$$
\left\{\left(y_{\xi}, 0\right) \in(\mathbb{Q} / \mathbb{Z})^{\left(P_{0} \times \omega\right)} \oplus \mathbb{Q}^{\left(P_{1}\right)}: \xi \in \cup_{n \in D} L_{n}\right\} \subset \varphi[G]
$$

onde $L_{1} \in\left[P_{1}\right]^{\mathfrak{c}}$ é tal que $\omega \cup\{\omega\} \subset L_{1}$ e $\left\{L_{n}: n \in D\right\}$ é uma família de elementos de $\left[P_{0}\right]^{\mathfrak{c}}$ dois a dois disjuntos satisfazendo:

- $\{\omega+n: n \in \mathbb{N}\} \cap \bigcup_{n \in D} L_{n}=\emptyset$;

- $\mathrm{o}\left(y_{\xi}\right)=n$, para todo $\xi \in L_{n}$ e todo $n \in D$;

- $\operatorname{supp} y_{\xi} \subset\{\xi\} \times \omega$, para todo $\xi \in \cup_{n \in D} L_{n}$.

Se $(H, J) \in(\mathbb{Q} / \mathbb{Z})^{\left(P_{0} \times \omega\right)} \oplus \mathbb{Q}^{\left(P_{1}\right)}$, então

$$
H(\xi, n)=\frac{p(H,(\xi, n))}{q(H,(\xi, n))}+\mathbb{Z}
$$

onde $p(H,(\xi, n)), q(H,(\xi, n)) \in \mathbb{Z}, q(H,(\xi, n))>0, \operatorname{mdc}(p(H,(\xi, n)), q(H,(\xi, n)))=1$ e $0 \leq p(H,(\xi, n)) \leq q(H,(\xi, n))-1$, para cada $(\xi, n) \in \operatorname{supp} H$. Além disso,

$$
J(\mu)=\frac{p(J, \mu)}{q(J, \mu)}
$$

onde $p(J, \mu), q(J, \mu) \in \mathbb{Z}, q(J, \mu)>0$ e $\operatorname{mdc}(p(J, \mu), q(J, \mu))=1$, para cada $\mu \in \operatorname{supp} J$.

Sejam

$$
|q(H)|=\max \{q(H,(\xi, n)):(\xi, n) \in \operatorname{supp} H\},
$$

\footnotetext{
${ }^{2}$ De acordo com os corolários 5.5.3 e 5.5.4, para cada número natural $n>1$ e para cada divisor $d$ de $n$, o conjunto $d G[n]$ é finito ou tem cardinalidade $\mathfrak{c}$ e $|G / T(G)|=\mathfrak{c}$.
} 


$$
|q(J)|=\max \{q(J, \mu): \mu \in \operatorname{supp} J\}
$$

e

$$
|p(J)|=\max \{|p(J, \mu)|: \mu \in \operatorname{supp} J\} .
$$

Denotaremos por $\mathcal{H}$ o conjunto de todas as funções

$$
\begin{aligned}
h: \omega & \rightarrow \varphi[G] \subset(\mathbb{Q} / \mathbb{Z})^{\left(P_{0} \times \omega\right)} \oplus \mathbb{Q}^{\left(P_{1}\right)} \\
n & \mapsto(g(n), f(n))
\end{aligned}
$$

que satisfazem uma das seguintes condições:

(1) $\operatorname{supp} f(n) \backslash \cup_{m<n} \operatorname{supp} f(m) \neq \emptyset$, para todo $n \in \omega$;

(2) $|q(f(n))|>n$, para todo $n \in \omega$;

(3) $\{|q(f(n))|: n \in \omega\}$ é limitado e $|p(f(n))|>n$, para todo $n \in \omega$;

(4) $|q(g(n))|>n$, para todo $n \in \omega$;

(5) (a) $\operatorname{supp} g(n) \backslash \cup_{m<n} \operatorname{supp} g(m) \neq \emptyset$, para todo $n \in \omega$;

(b) Existe $k \in D$ tal que o $(g(n))=k$, para todo $n \in \omega$;

(c) $f(n)=0$, para todo $n \in \omega$;

(d) $\{g(n): n \in \omega\}$ é um subconjunto $k$-round de $\varphi[G]$.

Sabemos que se $h: \omega \rightarrow \varphi[G] \subset(\mathbb{Q} / \mathbb{Z})^{\left(P_{0} \times \omega\right)} \oplus \mathbb{Q}^{\left(P_{1}\right)}$, então existem $j: \omega \rightarrow \omega$ estritamente crescente e $c \in \varphi[G]$ tais que a seqüência $n \mapsto(h \circ j)(n)+c$ é constante ou é de um dos tipos acima mencionados. Consideremos $\left\{h_{\xi}: \xi \in\left(L_{1} \cup \bigcup_{n \in D} L_{n}\right) \backslash \omega\right\}$ uma indexação de $\mathcal{H}$ tal que:

- $h_{\omega}(n)=\left(0, \chi_{n}\right)$, para todo $n \in \omega$;

- Se $\xi \in L_{1}$, então $h_{\xi}$ é do tipo $1,2,3$ ou 4;

- Se existe $k \in D$ tal que $\xi \in L_{k}$, então $h_{\xi}$ é do tipo 5 e o $\left(h_{\xi}(n)\right)=k$, para todo $n \in \omega$;

- $\cup_{n \in \omega} \operatorname{supp} h_{\xi}(n) \subset(\xi \times \omega) \cup \xi$, para todo $\xi \in\left(L_{1} \cup \cup_{n \in D} L_{n}\right) \backslash \omega$.

Sabemos também que se $\left\{p_{\xi}: \xi \in\left(L_{1} \cup \bigcup_{n \in D} L_{n}\right) \backslash \omega\right\}$ é uma família de ultrafiltros seletivos dois a dois incomparáveis segundo a ordem de Rudin-Keisler, então para cada $(H, J) \in$ $(\mathbb{Q} / \mathbb{Z})^{\left(P_{0} \times \omega\right)} \oplus \mathbb{Q}^{\left(P_{1}\right)} \backslash\{(0,0)\}$, existe $\phi_{(H, J)}:(\mathbb{Q} / \mathbb{Z})^{\left(P_{0} \times \omega\right)} \oplus \mathbb{Q}^{\left(P_{1}\right)} \rightarrow \mathbb{T}$ um homomorfismo de grupos tal que: 
- $\phi_{(H, J)}(H, J) \neq 0+\mathbb{Z}$;

- $\phi_{(H, J)}\left(0, \chi_{\xi}\right)=p_{\xi}-\lim \left\{\phi_{(H, J)}\left(h_{\xi}(n)\right): n \in \omega\right\}$, para cada $\xi \in L_{1} \backslash \omega$.

- $\phi_{(H, J)}\left(y_{\xi}, 0\right)=p_{\xi}-\lim \left\{\phi_{(H, J)}\left(h_{\xi}(n)\right): n \in \omega\right\}$, para cada $\xi \in \cup_{n \in D} L_{n} \backslash \omega$.

Em particular, temos que $\phi_{(H, J)}\left(0, \chi_{\omega}\right)=p_{\omega}-\lim \left\{\phi_{(H, J)}\left(0, \chi_{n}\right): n \in \omega\right\}$.

\subsection{A construção de uma nova topologia}

Fixemos $\kappa<2^{\mathfrak{c}}$ um cardinal e consideremos $\left\{\left(X_{\alpha}, \tau_{\alpha}\right): \alpha<\kappa\right\}$ uma família de espaços topológicos tal que $\left|X_{\alpha}\right|=\mathfrak{c}$, para todo $\alpha<\kappa$. Mostraremos que é possível munir $G$ de uma topologia que o torna um grupo topológico enumeravelmente compacto não homeomorfo a $\left(X_{\alpha}, \tau_{\alpha}\right)$, para todo $\alpha<\kappa$. Como estamos assumindo a existência de $2^{\mathfrak{c}}$ ultrafiltros seletivos, podemos tomar $\left\{p_{\xi}: \omega \leq \xi<\mathfrak{c}\right\} \subset \omega^{*}$ uma família de ultrafiltros seletivos dois a dois incomparáveis (segundo a ordem de Rudin-Keisler) tal que $p_{\omega} \notin \cup_{\alpha<\kappa} \mathcal{F}\left(X_{\alpha}\right){ }^{3}{ }^{3}$

Lema 6.3.1. Seja $A \in p_{\omega}$. Existe $\phi_{A}:(\mathbb{Q} / \mathbb{Z})^{\left(P_{0} \times \omega\right)} \oplus \mathbb{Q}^{\left(P_{1}\right)} \rightarrow \mathbb{T}$ um homomorfismo de grupos tal que:

(i) $\phi_{A}\left(0, \chi_{\xi}\right)=p_{\xi}-\lim \left\{\phi_{A}\left(h_{\xi}(n)\right): n \in \omega\right\}$, para cada $\xi \in L_{1} \backslash \omega$;

(ii) $\phi_{A}\left(y_{\xi}, 0\right)=p_{\xi}-\lim \left\{\phi_{A}\left(h_{\xi}(n)\right): n \in \omega\right\}$, para cada $\xi \in \bigcup_{n \in D} L_{n} \backslash \omega$;

(iii) $\left\{n \in \omega: \phi_{A}\left(h_{\omega}(n)\right) \in \Omega_{A}\right\}=A$, para alguma vizinhança $\Omega_{A}$ de $\phi_{A}\left(0, \chi_{\omega}\right)$ em $\mathbb{T}$.

Demonstração. Para cada $n \in \omega$, seja

$$
\phi\left(0, \chi_{n}\right)=\left\{\begin{array}{lll}
0+\mathbb{Z} & \text { se } & n \notin A \\
\frac{1}{2}+\mathbb{Z} & \text { se } & n \in A .
\end{array}\right.
$$

Façamos, ainda,

$$
\phi\left(0, \chi_{\omega}\right)=\frac{1}{2}+\mathbb{Z} .
$$

Temos que $\phi\left(0, \chi_{\omega}\right)=p_{\omega}-\lim \left\{\phi\left(h_{\omega}(n)\right): n \in \omega\right\}$ e $\left\{n \in \omega: \phi_{A}\left(h_{\omega}(n)\right) \in \Omega_{A}\right\}=A$, onde $\Omega_{A}$ denota o arco aberto de $\mathbb{T}$ centrado em $\frac{1}{2}+\mathbb{Z}$ com diâmetro $\frac{1}{4}$. Estendamos $\phi$ a um homomorfismo de grupos de $\left\{0_{(\mathbb{Q} / \mathbb{Z})^{\left(P_{0} \times \omega\right)}} \oplus \oplus \mathbb{Z}^{(\omega+1)}\right.$ em $\mathbb{T}$ e usemos o fato de $\mathbb{T}$ ser um

\footnotetext{
${ }^{3}$ Isto é possível, já que $\left|\cup_{\alpha<\kappa} \mathcal{F}\left(X_{\alpha}\right)\right| \leq \max \{\kappa, \mathfrak{c}\}<2^{c}$.
} 
grupo divisível para estender $\phi$ a um homomorfismo de grupos $\phi_{A} \Upsilon_{(\mathbb{Q} / \mathbb{Z})^{((\omega+\omega \backslash \omega+1) \times \omega)} \oplus \mathbb{Q}^{(\omega+1)}}$ : $(\mathbb{Q} / \mathbb{Z})^{((\omega+\omega \backslash \omega+1) \times \omega)} \oplus \mathbb{Q}^{(\omega+1)} \rightarrow \mathbb{T}$.

Consideremos $\left\{\alpha_{\xi}: \xi<\mathfrak{c}\right\}$ uma indexação estritamente crescente de $\mathfrak{c} \backslash(\omega+\omega)$.

- Se $\alpha_{0} \in P_{1} \backslash L_{1}$, usamos a divisibilidade de $\mathbb{T}$ para estender $\left.\phi_{A}\right\rceil_{(\mathbb{Q} / \mathbb{Z})^{((\omega+\omega \backslash \omega+1) \times \omega)} \oplus \mathbb{Q}^{(\omega+1)} \text { a }}$ um homomorfismo de grupos

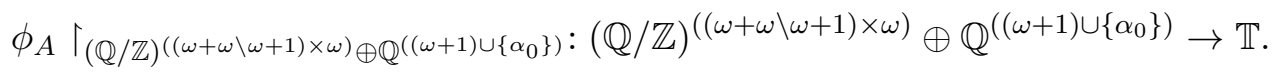

- Se $\alpha_{0} \in P_{0} \backslash \cup_{n \in D} L_{n}$, também usamos a divisibilidade de $\mathbb{T}$ para estender

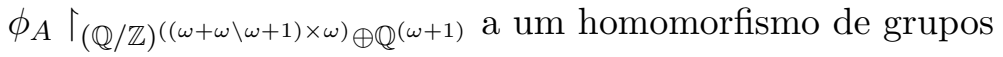

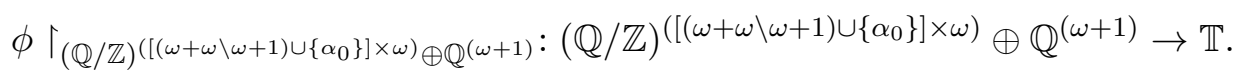

- Se $\alpha_{0} \in L_{1}$, definimos

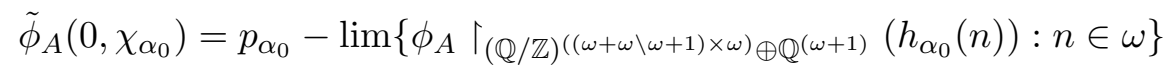

e façamos

$$
\tilde{\phi}_{A}(H, J)=\phi_{A} \Upsilon_{(\mathbb{Q} / \mathbb{Z})^{((\omega+\omega \backslash \omega+1) \times \omega)} \oplus \mathbb{Q}^{(\omega+1)}}(H, J)
$$

para todo $(H, J) \in(\mathbb{Q} / \mathbb{Z})^{((\omega+\omega \backslash \omega+1) \times \omega)} \oplus \mathbb{Q}^{(\omega+1)}$.

Seja $G_{0}$ o subgrupo de $\mathbb{Q}^{\left((\omega+1) \cup\left\{\alpha_{0}\right\}\right)}$ gerado por $\mathbb{Q}^{(\omega+1)} \cup\left\{\chi_{\alpha_{0}}\right\}$. Estendamos $\tilde{\phi}_{A}$ a um homomorfismo de grupos de $(\mathbb{Q} / \mathbb{Z})^{((\omega+\omega \backslash \omega+1) \times \omega)} \oplus G_{0}$ em $\mathbb{T}$ e usemos a divisibilidade de $\mathbb{T}$ para estender $\tilde{\phi}_{A}$ a um homomorfismo de grupos

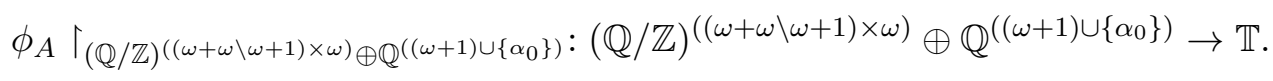

- Se $\alpha_{0} \in \cup_{n \in D} L_{n}$, definimos

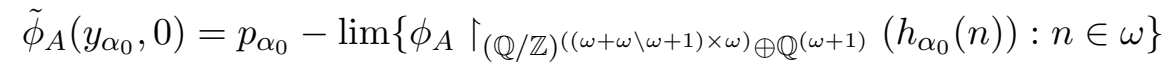

e façamos

$$
\tilde{\phi}_{A}(H, J)=\phi_{A} \Upsilon_{(\mathbb{Q} / \mathbb{Z})^{((\omega+\omega \backslash \omega+1) \times \omega)} \oplus \mathbb{Q}^{(\omega+1)}}(H, J)
$$

para todo $(H, J) \in(\mathbb{Q} / \mathbb{Z})^{((\omega+\omega \backslash \omega+1) \times \omega)} \oplus \mathbb{Q}^{(\omega+1)}$. 
Seja $G_{0}$ o subgrupo de $(\mathbb{Q} / \mathbb{Z})^{\left(\left[(\omega+\omega \backslash \omega+1) \cup\left\{\alpha_{0}\right\}\right] \times \omega\right)}$ gerado por $(\mathbb{Q} / \mathbb{Z})^{((\omega+\omega \backslash \omega+1) \times \omega)} \cup\left\{y_{\alpha_{0}}\right\}$. Estendamos $\tilde{\phi}_{A}$ a um homomorfismo de grupos de $G_{0} \oplus \mathbb{Q}^{(\omega+1)}$ em $\mathbb{T}$ e usemos a divisibilidade de $\mathbb{T}$ para estender $\tilde{\phi}_{A}$ a um homomorfismo de grupos

$$
\phi_{A} \Upsilon_{(\mathbb{Q} / \mathbb{Z})^{\left(\left[(\omega+\omega \backslash \omega+1) \cup\left\{\alpha_{0}\right\}\right] \times \omega\right)} \oplus \mathbb{Q}^{(\omega+1)}}:(\mathbb{Q} / \mathbb{Z})^{\left(\left[(\omega+\omega \backslash \omega+1) \cup\left\{\alpha_{0}\right\}\right] \times \omega\right)} \oplus \mathbb{Q}^{(\omega+1)} \rightarrow \mathbb{T} .
$$

Repetindo indutivamente esta construção, obteremos $\phi_{A}:(\mathbb{Q} / \mathbb{Z})^{\left(P_{0} \times \omega\right)} \oplus \mathbb{Q}^{\left(P_{1}\right)} \rightarrow \mathbb{T}$ um homomorfismo de grupos satisfazendo (i) e (ii).

Temos que

$$
\begin{aligned}
\Phi:(\mathbb{Q} / \mathbb{Z})^{\left(P_{0} \times \omega\right)} \oplus \mathbb{Q}^{\left(P_{1}\right)} & \rightarrow \mathbb{T}^{(\mathbb{Q} / \mathbb{Z})^{\left(P_{0} \times \omega\right)} \oplus \mathbb{Q}^{\left(P_{1}\right)} \backslash\{(0,0)\}} \\
(\tilde{H}, \tilde{J}) & \mapsto \Phi(\tilde{H}, \tilde{J})
\end{aligned}
$$

dada por

$$
\Phi(\tilde{H}, \tilde{J})(H, J)=\phi_{(H, J)}(\tilde{H}, \tilde{J}), \text { para cada }(H, J) \in(\mathbb{Q} / \mathbb{Z})^{\left(P_{0} \times \omega\right)} \oplus \mathbb{Q}^{\left(P_{1}\right)} \backslash\{(0,0)\}
$$

é um monomorfismo de grupos. Além disso,

$$
\begin{array}{cl}
\hat{\Phi}:(\mathbb{Q} / \mathbb{Z})^{\left(P_{0} \times \omega\right)} \oplus \mathbb{Q}^{\left(P_{1}\right)} & \rightarrow \mathbb{T}^{p_{\omega}} \\
(\tilde{H}, \tilde{J}) & \mapsto \hat{\Phi}(\tilde{H}, \tilde{J})
\end{array}
$$

dada por

$$
\hat{\Phi}(\tilde{H}, \tilde{J})(A)=\phi_{A}(\tilde{H}, \tilde{J}) \text {, para cada } A \in p_{\omega}
$$

é um homomorfismo de grupos. Logo,

$$
\begin{aligned}
\Psi:(\mathbb{Q} / \mathbb{Z})^{\left(P_{0} \times \omega\right)} \oplus \mathbb{Q}^{\left(P_{1}\right)} & \rightarrow \mathbb{T}^{(\mathbb{Q} / \mathbb{Z})^{\left(P_{0} \times \omega\right)} \oplus \mathbb{Q}^{\left(P_{1}\right)} \backslash\{(0,0)\}} \oplus \mathbb{T}^{p_{\omega}} \\
(\tilde{H}, \tilde{J}) & \mapsto(\Phi(\tilde{H}, \tilde{J}), \hat{\Phi}(\tilde{H}, \tilde{J}))
\end{aligned}
$$

é um monomorfismo de grupos.

Temos que

$$
\tau=\left\{\varphi^{-1} \circ \Psi^{-1}(U \cap \Psi[\varphi[G]]): U \text { é aberto em } \mathbb{T}^{(\mathbb{Q} / \mathbb{Z})^{\left(P_{0} \times \omega\right)} \oplus \mathbb{Q}^{\left(P_{1}\right)} \backslash\{(0,0)\}} \oplus \mathbb{T}^{p_{\omega}}\right\}
$$

é uma topologia em $G$ que o torna um grupo topológico. Além disso, temos que

$$
\varphi^{-1}\left(0, \chi_{\xi}\right)=p_{\xi}-\lim \left\{\varphi^{-1}\left(h_{\xi}(n)\right): n \in \omega\right\}, \text { se } \xi \in L_{1} \backslash \omega
$$


e

$$
\varphi^{-1}\left(y_{\xi}, 0\right)=p_{\xi}-\lim \left\{\varphi^{-1}\left(h_{\xi}(n)\right): n \in \omega\right\} \text {, se } \xi \in \cup_{n \in D} L_{n} \backslash \omega \text {. }
$$

Em particular, temos que

$$
\mathcal{F}\left(G,\left\{\varphi^{-1}\left(0, \chi_{n}\right): n \in \omega\right\}, \varphi^{-1}\left(0, \chi_{\omega}\right)\right) \subset p_{\omega}
$$

De fato, se $A \in \mathcal{F}\left(G,\left\{\varphi^{-1}\left(0, \chi_{n}\right): n \in \omega\right\}, \varphi^{-1}\left(0, \chi_{\omega}\right)\right)$, então existe $U$ vizinhança aberta de $\varphi^{-1}\left(0, \chi_{\omega}\right)$ em $G$ tal que $\left\{n \in \omega: \varphi^{-1}\left(h_{\omega}(n)\right) \in U\right\} \subset A$. Como $\varphi^{-1}\left(0, \chi_{\omega}\right)=$ $p_{\omega}-\lim \left\{\varphi^{-1}\left(h_{\omega}(n)\right): n \in \omega\right\}$, temos que $\left\{n \in \omega: \varphi^{-1}\left(h_{\omega}(n)\right) \in U\right\} \in p_{\omega}$ e, portanto, $A \in p_{\omega}$.

Observamos, ainda, que

$$
p_{\omega} \subset \mathcal{F}\left(G,\left\{\varphi^{-1}\left(0, \chi_{n}\right): n \in \omega\right\}, \varphi^{-1}\left(0, \chi_{\omega}\right)\right) .
$$

Com efeito, sejam $A \in p_{\omega}$ e $\Omega \subset \mathbb{T}^{(\mathbb{Q} / \mathbb{Z})^{\left(P_{0} \times \omega\right)} \oplus \mathbb{Q}^{\left(P_{1}\right)} \backslash\{(0,0)\}} \oplus \mathbb{T}^{p_{\omega}}$ tal que $\operatorname{proj}_{(H, J)}(\Omega)=\mathbb{T}$ para todo $(H, J) \in(\mathbb{Q} / \mathbb{Z})^{\left(P_{0} \times \omega\right)} \oplus \mathbb{Q}^{\left(P_{1}\right)} \backslash\{(0,0)\}, \operatorname{proj}_{\tilde{A}}(\Omega)=\mathbb{T}$ para todo $\tilde{A} \in p_{\omega} \backslash\{A\}$ e $\operatorname{proj}_{A}(\Omega)=\Omega_{A}$. Temos que $\Psi\left(0, \chi_{\omega}\right) \in \Omega$ e $\left\{n \in \omega: \varphi^{-1}\left(h_{\omega}(n)\right) \in \varphi^{-1} \circ \Psi^{-1}(\Omega)\right\}=\{n \in \omega:$ $\left.\Psi\left(h_{\omega}(n)\right) \in \Omega\right\}=\left\{n \in \omega: \Psi\left(h_{\omega}(n)\right)(A) \in \operatorname{proj}_{A}(\Omega)\right\}=\left\{n \in \omega: \phi_{A}\left(h_{\omega}(n)\right) \in \Omega_{A}\right\}=A$. Logo, $A \in \mathcal{F}\left(G,\left\{\varphi^{-1}\left(0, \chi_{n}\right): n \in \omega\right\}, \varphi^{-1}\left(0, \chi_{\omega}\right)\right)$.

Assim, $\mathcal{F}\left(G,\left\{\varphi^{-1}\left(0, \chi_{n}\right): n \in \omega\right\}, \varphi^{-1}\left(0, \chi_{\omega}\right)\right)=p_{\omega} \notin \cup_{\alpha<\kappa} \mathcal{F}\left(X_{\alpha}\right)$, o que implica que o grupo topológico $(G, \tau)$ não é homeomorfo a $\left(X_{\alpha}, \tau_{\alpha}\right)$, qualquer que seja $\alpha<\kappa$. Temos, portanto, o seguinte resultado.

Teorema 6.3.2. Se $G$ é um grupo abeliano de não torção e cardinalidade c que admite uma topologia de grupo enumeravelmente compacta, então $G$ admite $2^{\mathfrak{c}}$ topologias enumeravelmente compactas (duas a duas não homeomorfas) que o tornam um grupo topológico. 
CAPÍTULO 7

\section{Construindo uma topologia de grupo no grupo abeliano livre de cardinalidade $\mathfrak{c}$ que torna seu quadrado enumeravelmente compacto}

Neste capítulo, assumiremos a existência de $\mathfrak{c}$ ultrafiltros seletivos dois a dois incomparáveis (segundo a ordem de Rudin-Keisler) para mostrar que o grupo abeliano livre de cardinalidade $\mathfrak{c}$ pode ser munido de uma topologia de grupo que torna seu quadrado enumeravelmente compacto.

Para cada $J \in \mathbb{Q}^{(\mathfrak{c} \times \omega)}$ construiremos um homomorfismo de grupos $\phi_{J}: \mathbb{Q}^{(\mathfrak{c} \times \omega)} \rightarrow \mathbb{T}$ de modo que o produto diagonal da família $\left\{\phi_{J}: J \in \mathbb{Q}^{(\mathfrak{c} \times \omega)} \backslash\{0\}\right\}$ seja injetor e que a imagem de $\mathbb{Z}^{(\mathfrak{c} \times \omega)}$ pelo mesmo tenha quadrado enumeravelmente compacto quando munida da topologia de subespaço induzida por $\mathbb{T}^{\mathbb{Q}^{(\mathfrak{c} \times \omega)} \backslash\{0\}}$. A construção de tais homomorfismos continuará sendo feita em duas etapas: por indução, definiremos $\phi_{J}$ em um subgrupo enumerável de $\mathbb{Q}^{(\mathfrak{c} \times \omega)}$ e, em seguida, o estenderemos convenientemente a $\mathbb{Q}^{(\mathfrak{c} \times \omega)}$. Em cada passo da indução, aproximaremos os valores de $\phi_{J}$ por arcos abertos não vazios de $\mathbb{T}$. Para que isto se torne viável, lidaremos com três famílias de seqüências em $\mathbb{Q}^{(\mathfrak{c} \times \omega)}$ que possuem liberdade suficiente para terem atribuído a si um ponto de acumulação pré-determinado.

As famílias do tipo A são compostas por algumas seqüências cujos suportes são dois a dois disjuntos. O tratamento que daremos a elas é semelhante ao apresentado nos capítulos anteriores desta tese. São necessárias, contudo, novas idéias para tratar das famílias do tipo B e C, uma vez que estas possuem um par $\left\{f_{0}, f_{1}\right\}$ de seqüências em $\mathbb{Q}^{(\mathfrak{c} \times \omega)}$ cujos suportes não são disjuntos e que, portanto, não podem ser tratados independentemente. 
Para cada $n \in \omega$, fixaremos $\left(\theta_{n}, m_{n}\right) \in \operatorname{supp} f_{0}(n) \cap \operatorname{supp} f_{1}(n)$ e consideraremos a seqüência $\left\{a_{n}: n \in \omega\right\}$ dada por

$$
a_{n}=\frac{\left|f_{0}(n)\left(\theta_{n}, m_{n}\right)\right|}{\left|f_{1}(n)\left(\theta_{n}, m_{n}\right)\right|}
$$

As famílias do tipo $\mathrm{B}$ estão relacionadas à convergência da seqüência $\left\{a_{n}: n \in \omega\right\}$ para 0 e as famílias do tipo $\mathrm{C}$ estão relacionadas à convergência de $\left\{a_{n}: n \in \omega\right\}$ para um número irracional. No primeiro caso, trataremos de $f_{0}$ e $f_{1}$ separadamente, enquanto no segundo, usaremos o teorema de Kronecker para lidar simultaneamente com ambas.

Se a seqüência $\left\{a_{n}: n \in \omega\right\}$ convergir para um número racional não nulo, usaremos a caracterização das soluções gerais de equações diofantinas lineares em duas variáveis para obter uma família do tipo A ou teremos de escrever uma combinação linear de elementos de $\mathbb{Z}^{(\mathfrak{c} \times \omega)}$ com coeficientes racionais. É por esta razão que trabalhamos com seqüências em $\mathbb{Q}^{(\mathfrak{c} \times \omega)}$ em vez de $\mathbb{Z}^{(\mathfrak{c} \times \omega)}$.

\subsection{Uma escolha adequada de seqüências}

Se $J \in \mathbb{Q}^{(\mathfrak{c} \times \omega)}$, então

$$
J=\sum_{(\mu, k) \in \operatorname{supp} J} J(\mu, k) \cdot \chi_{(\mu, k)}
$$

onde $\chi_{(\mu, k)}: \mathfrak{c} \times \omega \rightarrow \mathbb{Q}$ é tal que

$$
\chi_{(\mu, k)}(\xi, n)=\left\{\begin{array}{lll}
1 & \text { se } & (\xi, n)=(\mu, k) \\
0 & \text { se } & (\xi, n) \neq(\mu, k)
\end{array}\right.
$$

Se $(\mu, k) \in \operatorname{supp} J$, podemos escrever

$$
J(\mu, k)=\frac{p(J,(\mu, k))}{q(J,(\mu, k))}
$$

onde $p(J,(\mu, k)), q(J,(\mu, k)) \in \mathbb{Z}, q(J,(\mu, k))>0$ e $\operatorname{mdc}(p(J,(\mu, k)), q(J,(\mu, k)))=1$. Seja

$$
b(J)=\operatorname{mmc}\{q(J,(\mu, k)):(\mu, k) \in \operatorname{supp} J\}
$$


e, para cada $(\mu, k) \in \operatorname{supp} J$, seja

$$
a(J,(\mu, k))=p(J,(\mu, k)) \cdot \frac{b(J)}{q(J,(\mu, k))} .
$$

Seja, ainda,

$$
|a(J)|=\max \{|a(J,(\mu, k))|:(\mu, k) \in \operatorname{supp} J\} .
$$

Por fim, se $X \subset \mathfrak{c} \times \omega$, então

$$
\pi_{1}[X]=\{\xi<\mathfrak{c}: \exists n \in \omega \text { tal que }(\xi, n) \in X\}
$$

$\mathrm{e}$

$$
\pi_{2}[X]=\{n \in \omega: \exists \xi<\mathfrak{c} \text { tal que }(\xi, n) \in X\} .
$$

Definição 7.1.1. Seja $f: \omega \rightarrow \mathbb{Z}^{(\mathfrak{c} \times \omega)}$. Dizemos que $f$ é do tipo 1 se $\operatorname{supp} f(n) \backslash$ $\cup_{m<n} \operatorname{supp} f(m) \neq \emptyset$ para cada $n \in \omega$. Dizemos que $f$ é do tipo 2 se $|f(n)|>n$ para cada $n \in \omega$, onde $|f(n)|=\max \{|f(n)(\mu, k)|:(\mu, k) \in \operatorname{supp} f(n)\}$.

A proposição abaixo encontra-se em [30]. Como sua demonstração é curta, optamos por reproduzi-la aqui.

Proposição 7.1.2. [30] Se $f: \omega \rightarrow \mathbb{Z}^{(\mathfrak{c} \times \omega)}$, então existe $j: \omega \rightarrow \omega$ estritamente crescente tal que $f \circ j$ é constante ou é de um dos tipos acima mencionados.

Demonstração. Há três casos a serem considerados.

Caso 1: $\cup_{n \in \omega} \operatorname{supp} f(n)$ é infinito.

Seja $n_{0}=0$. Fixemos $k \in \omega$ e suponhamos definidos $n_{0}, n_{1}, \ldots, n_{k}$. Uma vez que $\cup_{n \in \omega} \operatorname{supp} f(n)$ é infinito, existe um número natural $n_{k+1}>n_{k}$ tal que $\operatorname{supp} f\left(n_{k+1}\right) \backslash$ $\cup_{l<k+1} \operatorname{supp} f\left(n_{l}\right) \neq \emptyset$. Temos que

$$
\begin{aligned}
j: \omega & \rightarrow \omega \\
k & \mapsto n_{k}
\end{aligned}
$$

é uma função estritamente crescente tal que $f \circ j$ é do tipo 1 .

Caso 2: $\cup_{n \in \omega} \operatorname{supp} f(n)$ é finito e $\{|f(n)|: n \in \omega\}$ é ilimitado.

Por indução, tomemos $\left\{n_{k}: k \in \omega\right\}$ uma seqüência estritamente crescente de números 
naturais tal que $\left|f\left(n_{k}\right)\right|>k$, para todo $k \in \omega$. Temos que

$$
\begin{aligned}
j: \omega & \rightarrow \omega \\
k & \mapsto n_{k}
\end{aligned}
$$

é uma função estritamente crescente tal que $f \circ j$ é do tipo 2 .

Caso 3: $\cup_{n \in \omega} \operatorname{supp} f(n)$ é finito e $\{|f(n)|: n \in \omega\}$ é limitado.

Temos que $\{f(n): n \in \omega\}$ é um conjunto finito e, portanto, $f$ possui uma subseqüência constante.

Definição 7.1.3. Seja $F=\left\{f_{0}, \ldots, f_{k}\right\}$ uma família finita de seqüências em $\mathbb{Q}^{(\mathfrak{c} \times \omega)}$. Dizemos que $F$ é do tipo A se:

- $f_{i}(n) \in \mathbb{Z}^{(\mathfrak{c} \times \omega)}$ para todo $n \in \omega$ e todo $i \in\{0, \ldots, k\}$;

- $f_{i}$ é do tipo 1 ou 2, para todo $i \in\{0, \ldots, k\}$;

- $\operatorname{supp} f_{i}(n) \cap \operatorname{supp} f_{j}(n)=\emptyset$ para todo $n \in \omega$ e para quaisquer $i, j \in\{0, \ldots, k\}$ com $i \neq j$.

Se $F$ é do tipo $A$, façamos $d(F)=1$. Dizemos que $F$ é do tipo B se:

- $f_{i}(n) \in \mathbb{Z}^{(\mathfrak{c} \times \omega)}$ para todo $n \in \omega$ e todo $i \in\{2, \ldots, k\}$;

- $f_{i}$ é do tipo 1 ou 2, para todo $i \in\{2, \ldots, k\}$;

- $f_{0}(n)=\frac{1}{d(F)} \cdot \tilde{f}_{0}(n)$ e $f_{1}(n)=\frac{1}{d(F)} \cdot \tilde{f}_{1}(n)$ para cada $n \in \omega$, onde $\tilde{f}_{0}: \omega \rightarrow \mathbb{Z}^{(\mathfrak{c} \times \omega)}$ é do tipo 1 ou $2, \tilde{f}_{1}: \omega \rightarrow \mathbb{Z}^{(\mathfrak{c} \times \omega)}$ é do tipo 1 ou 2 e $d(F)$ é um inteiro positivo;

- $\operatorname{supp} f_{0}(n) \subset \operatorname{supp} f_{1}(n)$, para todo $n \in \omega$;

- $\operatorname{supp} f_{i}(n) \cap \operatorname{supp} f_{j}(n)=\emptyset$ para todo $n \in \omega$ e para quaisquer $i, j \in\{2, \ldots, k\}$ com $i \neq j$;

- $\operatorname{supp} f_{i}(n) \cap \operatorname{supp} f_{j}(n)=\emptyset$ para todo $n \in \omega$, para todo $i \in\{0,1\}$ e todo $j \in\{2, \ldots, k\}$;

- Para cada $n \in \omega$, existe $\left(\theta_{n}, m_{n}\right) \in \operatorname{supp} f_{0}(n)$ tal que a seqüência

$$
\left\{\frac{f_{0}(n)\left(\theta_{n}, m_{n}\right)}{f_{1}(n)\left(\theta_{n}, m_{n}\right)}: n \in \omega\right\}
$$

é estritamente monótona e converge para 0. 
Dizemos que F é do tipo C se:

- $f_{i}(n) \in \mathbb{Z}^{(\mathfrak{c} \times \omega)}$ para todo $n \in \omega$ e todo $i \in\{0, \ldots, k\}$;

- $f_{i}$ é do tipo 1 ou 2, para todo $i \in\{0, \ldots, k\}$;

- $\operatorname{supp} f_{0}(n)=\operatorname{supp} f_{1}(n)$, para todo $n \in \omega$;

- $\operatorname{supp} f_{i}(n) \cap \operatorname{supp} f_{j}(n)=\emptyset$ para todo $n \in \omega$ e para quaisquer $i, j \in\{2, \ldots, k\}$ com $i \neq j$;

- $\operatorname{supp} f_{i}(n) \cap \operatorname{supp} f_{j}(n)=\emptyset$ para todo $n \in \omega$, para todo $i \in\{0,1\}$ e todo $j \in\{2, \ldots, k\}$;

- Para cada $n \in \omega$, existe $\left(\theta_{n}, m_{n}\right) \in \operatorname{supp} f_{0}(n)$ tal que a seqüência

$$
\left\{\frac{f_{0}(n)\left(\theta_{n}, m_{n}\right)}{f_{1}(n)\left(\theta_{n}, m_{n}\right)}: n \in \omega\right\}
$$

é estritamente monótona e converge para algum $\zeta \in \mathbb{R} \backslash \mathbb{Q}$.

Se $F$ é do tipo $C$, façamos $d(F)=1$.

$\mathrm{O}$ conjunto $\mathcal{F}$ de todas as famílias do tipo $\mathrm{A}, \mathrm{B}$ ou $\mathrm{C}$ nos permite recuperar uma subseqüência de um par qualquer de seqüências em $\mathbb{Z}^{(\mathfrak{c} \times \omega)}$.

Proposição 7.1.4. Sejam $g, h: \omega \rightarrow \mathbb{Z}^{(\mathfrak{c} \times \omega)}$. Existem $F \in \mathcal{F}, j: \omega \rightarrow \omega$ estritamente crescente e $\tilde{g}, \tilde{h} \in \mathbb{Z}^{(\mathfrak{c} \times \omega)}$ tais que $(g \circ j)(n)=\tilde{g}+\sum_{f \in F} a_{f} f(n) e(h \circ j)(n)=\tilde{h}+\sum_{f \in F} b_{f} f(n)$ para todo $n \in \omega$, onde $a_{f}, b_{f} \in \mathbb{Z}$ para todo $f \in F$.

Demonstração. Consideremos $g_{0}, g_{1}, h_{0}, h_{1}: \omega \rightarrow \mathbb{Z}^{(\mathfrak{c} \times \omega)}$ dadas por

$$
\begin{aligned}
& g_{0}(n)=\sum_{(\mu, k) \in \operatorname{supp} g(n) \backslash \operatorname{supp} h(n)} g(n)(\mu, k) \cdot \chi_{(\mu, k)}, \\
& g_{1}(n)=\sum_{(\mu, k) \in \operatorname{supp} g(n) \cap \operatorname{supp} h(n)} g(n)(\mu, k) \cdot \chi_{(\mu, k)}, \\
& h_{0}(n)=\sum_{(\mu, k) \in \operatorname{supp} h(n) \backslash \operatorname{supp} g(n)} h(n)(\mu, k) \cdot \chi_{(\mu, k)}
\end{aligned}
$$

$\mathrm{e}$

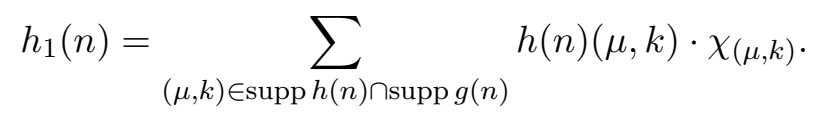


Temos que $g(n)=g_{0}(n)+g_{1}(n)$ e $h(n)=h_{0}(n)+h_{1}(n)$, para todo $n \in \omega$.

Da proposição 7.1.2 segue que existe $j_{1}: \omega \rightarrow \omega$ estritamente crescente tal que $g_{0} \circ j_{1}, g_{1} \circ j_{1}$, $h_{0} \circ j_{1}$ e $h_{1} \circ j_{1}$ são do tipo 1,2 , ou constante. Se $g_{1} \circ j_{1}$ ou $h_{1} \circ g_{1}$ for constante, é possível tomar $F \in\left[{ }^{\omega} \mathbb{Q}^{(\mathfrak{c} \times \omega)}\right]^{<\omega}$ do tipo $A$ e $\tilde{g}, \tilde{h} \in \mathbb{Z}^{(\mathfrak{c} \times \omega)}$ tais que $\left(g \circ j_{1}\right)(n)=\tilde{g}+\sum_{f \in F} a_{f} f(n)$ e $\left(h \circ j_{1}\right)(n)=\tilde{h}+\sum_{f \in F} b_{f} f(n)$ para todo $n \in \omega$, onde $a_{f}, b_{f} \in \mathbb{Z}$ para todo $f \in F$. Podemos, portanto, supor que $g_{1} \circ j_{1}$ e $h_{1} \circ g_{1}$ são do tipo 1 ou 2 .

Seja

$$
A=\left\{\frac{\left(g_{1} \circ j_{1}\right)(n)(\mu, k)}{\left(h_{1} \circ j_{1}\right)(n)(\mu, k)}:(\mu, k) \in \operatorname{supp}\left(g_{1} \circ j_{1}\right)(n)=\operatorname{supp}\left(h_{1} \circ j_{1}\right)(n), n \in \omega\right\} .
$$

Se $A$ é finito - digamos $A=\left\{\frac{p_{0}}{q_{0}}, \ldots, \frac{p_{k}}{q_{k}}\right\}$, onde $p_{i}, q_{i} \in \mathbb{Z} \backslash\{0\}, q_{i}>0$ e $\operatorname{mdc}\left(p_{i}, q_{i}\right)=1$ para todo $i \in\{0, \ldots, k\}$ - consideremos

$$
g_{1, i}(n)=\sum_{\substack{(\mu, k) \in \operatorname{supp}\left(g_{1} \circ j_{1}\right)(n), \frac{\left(g_{1} \circ j_{1}\right)(n)(\mu, k)}{\left(h_{1} \circ j_{1}\right)(n)(\mu, k)}=\frac{p_{i}}{q_{i}}}}\left(g_{1} \circ j_{1}\right)(n)(\mu, k) \cdot \chi_{(\mu, k)}
$$

e

$$
h_{1, i}(n)=\sum_{\substack{(\mu, k) \in \operatorname{supp}\left(h_{1} \circ j_{1}\right)(n), \frac{\left(g_{1} \circ j_{1}\right)(n)(\mu, k)}{\left(h_{1} \circ j_{1}\right)(n)(\mu, k)}=\frac{p_{i}}{q_{i}}}}\left(h_{1} \circ j_{1}\right)(n)(\mu, k) \cdot \chi_{(\mu, k)}
$$

para cada $n \in \omega$ e cada $i \in\{0, \ldots, k\}$. Note que

$$
\left(g_{1} \circ j_{1}\right)(n)=\sum_{i=0}^{k} g_{1, i}(n)
$$

$\mathrm{e}$

$$
\left(h_{1} \circ j_{1}\right)(n)=\sum_{i=0}^{k} h_{1, i}(n) .
$$

Se $i \in\{0, \ldots, k\}$ e $n \in \omega$, então $\operatorname{supp} g_{1, i}(n)=\operatorname{supp} h_{1, i}(n)$. Além disso,

$$
\frac{g_{1, i}(n)(\mu, k)}{h_{1, i}(n)(\mu, k)}=\frac{p_{i}}{q_{i}}
$$

para cada $(\mu, k) \in \operatorname{supp} g_{1, i}(n)=\operatorname{supp} h_{1, i}(n)$. Como $\operatorname{mdc}\left(p_{i}, q_{i}\right)=1$, temos que $q_{i} \mid h_{1, i}(n)(\mu, k)$ 
para todo $(\mu, k) \in \operatorname{supp} g_{1, i}(n)=\operatorname{supp} h_{1, i}(n)$. Seja $f_{i}: \omega \rightarrow \mathbb{Z}^{(\mathfrak{c} \times \omega)}$ dada por

$$
f_{i}(n)(\mu, k)=\frac{h_{1, i}(n)(\mu, k)}{q_{i}}
$$

para cada $(\mu, k) \in \mathfrak{c} \times \omega$ e cada $n \in \omega$. Temos que

$$
\left(g_{1} \circ j_{1}\right)(n)=\sum_{i=0}^{k} p_{i} \cdot f_{i}(n)
$$

$\mathrm{e}$

$$
\left(h_{1} \circ j_{1}\right)(n)=\sum_{i=0}^{k} q_{i} \cdot f_{i}(n) .
$$

Tomemos $j_{2}: \omega \rightarrow \omega$ estritamente crescente tal que $f_{i} \circ j_{2}$ é do tipo 1, 2 ou constante, para cada $i \in\{0, \ldots, k\}$. Dado $i \in\{0, \ldots, k\}$, definimos $s_{i}=f_{i} \circ j_{2}$. Sejam $s_{k+1}=g_{0} \circ j_{1} \circ j_{2} \mathrm{e}$ $s_{k+2}=h_{0} \circ j_{1} \circ j_{2}$. Consideremos $\left\{n_{0}, \ldots, n_{l}\right\}$ uma enumeração estritamente crescente do conjunto $I=\left\{i \in\{0, \ldots, k\}: s_{i}\right.$ não é constante $\}$. Se $s_{k+1}$ e $s_{k+2}$ são constantes, façamos $F=\left\{s_{n_{i}}: 0 \leq\right.$ $i \leq l\}$; se $s_{k+1}$ é constante e $s_{k+2}$ não é constante, façamos $F=\left\{s_{n_{i}}: 0 \leq i \leq l\right\} \cup\left\{s_{k+2}\right\}$; se $s_{k+1}$ não é constante e $s_{k+2}$ é constante, façamos $F=\left\{s_{n_{i}}: 0 \leq i \leq l\right\} \cup\left\{s_{k+1}\right\}$; se $s_{k+1}$ e $s_{k+2}$ não são constantes, façamos $F=\left\{s_{n_{i}}: 0 \leq i \leq l\right\} \cup\left\{s_{k+1}\right\} \cup\left\{s_{k+2}\right\}$. Temos que $F$ é do tipo $A$ e, portanto, pertence a $\mathcal{F}$. Observamos que

$$
\left(g \circ j_{1} \circ j_{2}\right)(n)=\sum_{i \in\{0, \ldots, k\} \backslash I} s_{i}(0)+s_{k+1}(n)+\sum_{i=0}^{l} p_{n_{i}} \cdot s_{n_{i}}
$$

$\mathrm{e}$

$$
\left(h \circ j_{1} \circ j_{2}\right)(n)=\sum_{i \in\{0, \ldots, k\} \backslash I} s_{i}(0)+s_{k+2}(n)+\sum_{i=0}^{l} q_{n_{i}} \cdot s_{n_{i}}
$$

para todo $n \in \omega$.

Se $A$ é infinito, existem $j_{2}: \omega \rightarrow \omega$ estritamente crescente e $\left\{\left(\theta_{n}, m_{n}\right): n \in \omega\right\} \subset \mathfrak{c} \times \omega$ tais que $g_{1} \circ j_{1} \circ j_{2}$ e $h_{1} \circ j_{1} \circ j_{2}$ são do tipo 1 ou $2,\left(\theta_{n}, m_{n}\right) \in \operatorname{supp}\left(g_{1} \circ j_{1} \circ j_{2}\right)(n) \cap \operatorname{supp}\left(h_{1} \circ j_{1} \circ j_{2}\right)(n)$ para todo $n \in \omega$,

$$
\frac{\left(g_{1} \circ j_{1} \circ j_{2}\right)(n)\left(\theta_{n}, m_{n}\right)}{\left(h_{1} \circ j_{1} \circ j_{2}\right)(n)\left(\theta_{n}, m_{n}\right)} \rightarrow \zeta
$$


para algum $\zeta \in[-\infty,+\infty]$, com

$$
\left\{\frac{\left(g_{1} \circ j_{1} \circ j_{2}\right)(n)\left(\theta_{n}, m_{n}\right)}{\left(h_{1} \circ j_{1} \circ j_{2}\right)(n)\left(\theta_{n}, m_{n}\right)}: n \in \omega\right\}
$$

estritamente monótona.

Se $\zeta=0$, consideremos $s_{0}=g_{1} \circ j_{1} \circ j_{2}, s_{1}=h_{1} \circ j_{1} \circ j_{2}, s_{2}=g_{0} \circ j_{1} \circ j_{2}$ e $s_{3}=h_{0} \circ j_{1} \circ j_{2}$. Seja $I=\left\{i \in\{0,1,2,3\}: s_{i}\right.$ não é constante $\}$. Observe que $0,1 \in I$. Se $2,3 \in I$, então $F=\left\{s_{0}, s_{1}, s_{2}, s_{3}\right\}$ é do tipo $B$ e, portanto, pertence a $\mathcal{F}$. Tomemos $j=j_{1} \circ j_{2}$ e $\tilde{g}=\tilde{h}=0$. Temos que $(g \circ j)(n)=s_{0}(n)+s_{2}(n)$ e $(h \circ j)(n)=s_{1}(n)+s_{3}(n)$ para todo $n \in \omega$. Se $2 \in I$ e $3 \notin I$, façamos $F=\left\{s_{0}, s_{1}, s_{2}\right\}, j=j_{1} \circ j_{2}, \tilde{g}=0$ e $\tilde{h}=s_{3}(0)$. Se $2 \notin I$ e $3 \in I$, façamos $F=\left\{s_{0}, s_{1}, s_{3}\right\}, j=j_{1} \circ j_{2}, \tilde{g}=s_{2}(0)$ e $\tilde{h}=0$. Por fim, se $2,3 \notin I$, façamos $F=\left\{s_{0}, s_{1}\right\}$, $j=j_{1} \circ j_{2}, \tilde{g}=s_{2}(0)$ e $\tilde{h}=s_{3}(0)$.

Se $\zeta=-\infty$ ou $\zeta=+\infty$, então

$$
\frac{\left(h_{1} \circ j_{1} \circ j_{2}\right)(n)\left(\theta_{n}, m_{n}\right)}{\left(g_{1} \circ j_{1} \circ j_{2}\right)(n)\left(\theta_{n}, m_{n}\right)} \rightarrow 0
$$

Procedamos, agora, como no caso $\zeta=0$.

Se $\zeta \in \mathbb{R} \backslash \mathbb{Q}$, consideremos $s_{0}=g_{1} \circ j_{1} \circ j_{2}, s_{1}=h_{1} \circ j_{1} \circ j_{2}, s_{2}=g_{0} \circ j_{1} \circ j_{2}$ e $s_{3}=h_{0} \circ j_{1} \circ j_{2}$. Seja $I=\left\{i \in\{0,1,2,3\}: s_{i}\right.$ não é constante $\}$. Observe que $0,1 \in I$. Se $2,3 \in I$, então $F=\left\{s_{0}, s_{1}, s_{2}, s_{3}\right\}$ é do tipo $C$ e, portanto, pertence a $\mathcal{F}$. Tomemos $j=j_{1} \circ j_{2}$ e $\tilde{g}=\tilde{h}=0$. Temos que $(g \circ j)(n)=s_{0}(n)+s_{2}(n)$ e $(h \circ j)(n)=s_{1}(n)+s_{3}(n)$ para todo $n \in \omega$. Os outros casos $(2 \in I$ e $3 \notin I, 2 \notin I$ e $3 \in I, 2,3 \notin I)$ são tratados de maneira análoga.

Se $\zeta \in \mathbb{Q} \backslash\{0\}$, então $\zeta=\frac{p}{q}$, onde $p, q \in \mathbb{Z} \backslash\{0\}, q>0$ e $\operatorname{mdc}(p, q)=1$. Consideremos

$$
r_{0}(n)=\frac{q \cdot\left(g_{1} \circ j_{1} \circ j_{2}\right)(n)-p \cdot\left(h_{1} \circ j_{1} \circ j_{2}\right)(n)}{q}
$$

$\mathrm{e}$

$$
r_{1}(n)=\frac{\left(h_{1} \circ j_{1} \circ j_{2}\right)(n)}{q}
$$

para cada $n \in \omega$. Além disso, sejam $r_{2}=g_{0} \circ j_{1} \circ j_{2}$ e $r_{3}=h_{0} \circ j_{1} \circ j_{2}$. Existe $j_{3}: \omega \rightarrow \omega$ estritamente crescente tal que

$$
n \mapsto q \cdot\left(g_{1} \circ j_{1} \circ j_{2} \circ j_{3}\right)(n)-p \cdot\left(h_{1} \circ j_{1} \circ j_{2} \circ j_{3}\right)(n)
$$


é do tipo 1, 2 ou constante. Se tal seqüência for do tipo 1 ou 2 , façamos $\tilde{s}_{0}=q \cdot\left(g_{1} \circ j_{1} \circ\right.$ $\left.j_{2} \circ j_{3}\right)-p \cdot\left(h_{1} \circ j_{1} \circ j_{2} \circ j_{3}\right), \tilde{s}_{1}=h_{1} \circ j_{1} \circ j_{2} \circ j_{3}, s_{2}=r_{2} \circ j_{3}$ e $s_{3}=r_{3} \circ j_{3}$. Seja $I=\left\{i \in\{2,3\}: s_{i}\right.$ não é constante $\}$. Se $2,3 \in I$, então $F=\left\{s_{0}, s_{1}, s_{2}, s_{3}\right\}$ é do tipo $B$, onde $s_{0}(n)=\frac{1}{q} \cdot \tilde{s}_{0}(n)$ e $s_{1}(n)=\frac{1}{q} \cdot \tilde{s}_{1}(n)$ para todo $n \in \omega$. Tomemos $j=j_{1} \circ j_{2} \circ j_{3}, \tilde{g}=0$ e $\tilde{h}=0$. Temos que $(g \circ j)(n)=s_{0}(n)+p \cdot s_{1}(n)+s_{2}(n)$ e $(h \circ j)(n)=q \cdot s_{1}(n)+s_{3}(n)$ para todo $n \in \omega$. Os outros casos $(2 \in I$ e $3 \notin I, 2 \notin I$ e $3 \in I, 2,3 \notin I)$ são tratados de maneira análoga.

Se a seqüência

$$
n \mapsto q \cdot\left(g_{1} \circ j_{1} \circ j_{2} \circ j_{3}\right)(n)-p \cdot\left(h_{1} \circ j_{1} \circ j_{2} \circ j_{3}\right)(n)
$$

é constante, existe $J \in \mathbb{Z}^{(\mathfrak{c} \times \omega)}$ tal que

$$
q \cdot\left(g_{1} \circ j_{1} \circ j_{2} \circ j_{3}\right)(n)(\mu, k)-p \cdot\left(h_{1} \circ j_{1} \circ j_{2} \circ j_{3}\right)(n)(\mu, k)=J(\mu, k)
$$

para todo $(\mu, k) \in \mathfrak{c} \times \omega$ e todo $n \in \omega$.

Fixemos $(\mu, k) \in \mathfrak{c} \times \omega$. Como $\operatorname{mdc}(p, q)=1$, a equação diofantina $q x-p y=J(\mu, k)$ tem infinitas soluções. Se $x=x_{(\mu, k)}, y=y_{(\mu, k)}$ é uma solução particular da mesma, então todas as suas soluções são da forma $x=x_{(\mu, k)}-p t, y=y_{(\mu, k)}-q t$, para $t \in \mathbb{Z}$. Logo, para cada $n \in \omega$ e cada $(\mu, k) \in \operatorname{supp}\left(g_{1} \circ j_{1} \circ j_{2} \circ j_{3}\right)(n)=\operatorname{supp}\left(g_{1} \circ j_{1} \circ j_{2} \circ j_{3}\right)(n)$, existe $t_{n,(\mu, k)} \in \mathbb{Z}$ tal que

$$
\left(g_{1} \circ j_{1} \circ j_{2} \circ j_{3}\right)(n)(\mu, k)=x_{(\mu, k)}-p t_{n,(\mu, k)}
$$

$\mathrm{e}$

$$
\left(h_{1} \circ j_{1} \circ j_{2} \circ j_{3}\right)(n)(\mu, k)=y_{(\mu, k)}-q t_{n,(\mu, k)} .
$$

Observe que se $(\mu, k) \notin \operatorname{supp} J$, podemos tomar $x_{(\mu, k)}=0, y_{(\mu, k)}=0$. Destacamos, ainda, que se $p, q>0$, então é possível tomar $x_{(\mu, k)}, y_{(\mu, k)} \geq 0 .{ }^{1}$ Portanto, para cada $n \in \omega$, temos que

$$
\left(g_{1} \circ j_{1} \circ j_{2} \circ j_{3}\right)(n)=\sum_{(\mu, k) \in \operatorname{supp} J} x_{(\mu, k)} \cdot \chi_{(\mu, k)}+p \cdot \sum_{(\mu, k) \in \operatorname{supp}\left(g_{1} \circ j_{1} \circ j_{2} \circ j_{3}\right)(n)}-t_{n,(\mu, k)} \cdot \chi_{(\mu, k)}
$$

$\mathrm{e}$

$$
\left(h_{1} \circ j_{1} \circ j_{2} \circ j_{3}\right)(n)=\sum_{(\mu, k) \in \operatorname{supp} J} y_{(\mu, k)} \cdot \chi_{(\mu, k)}+q \cdot \sum_{(\mu, k) \in \operatorname{supp}\left(h_{1} \circ j_{1} \circ j_{2} \circ j_{3}\right)(n)}-t_{n,(\mu, k)} \cdot \chi_{(\mu, k)} .
$$

\footnotetext{
${ }^{1}$ Isto será importante na construção de um semigrupo de Wallace cujo quadrado é enumeravelmente compacto.
} 
Além disso,

$$
n \mapsto \sum_{(\mu, k) \in \operatorname{supp} J} x_{(\mu, k)} \cdot \chi_{(\mu, k)}
$$

$\mathrm{e}$

$$
n \mapsto \sum_{(\mu, k) \in \operatorname{supp} J} y_{(\mu, k)} \cdot \chi_{(\mu, k)}
$$

são constantes.

Tomemos $j_{4}: \omega \rightarrow \omega$ estritamente crescente tal que a seqüência $s_{0}: \omega \rightarrow \mathbb{Z}^{(\mathfrak{c} \times \omega)}$ dada por

$$
s_{0}(n)=\sum_{(\mu, k) \in \operatorname{supp}\left(g_{1} \circ j_{1} \circ j_{2} \circ j_{3} \circ j_{4}\right)(n)}-t_{n,(\mu, k)} \cdot \chi_{(\mu, k)}
$$

seja do tipo 1 ou 2 e definamos $j=j_{1} \circ j_{2} \circ j_{3} \circ j_{4}, \tilde{g}=\sum_{(\mu, k) \in \operatorname{supp} J} x_{(\mu, k)} \cdot \chi_{(\mu, k)}$, $\tilde{h}=\sum_{(\mu, k) \in \operatorname{supp} J} y_{(\mu, k)} \cdot \chi_{(\mu, k)}, s_{1}=g_{0} \circ j$ e $s_{2}=h_{0} \circ j$. Consideremos $I=\{i \in\{1,2\}$ : $s_{i}$ não é constante $\}$. Se $1,2 \in I$, então $F=\left\{s_{0}, s_{1}, s_{2}\right\}$ é do tipo A e, portanto, pertence a $\mathcal{F}$. Além disso, $(g \circ j)(n)=\tilde{g}+p \cdot s_{0}(n)+s_{1}(n)$ e $(h \circ j)(n)=\tilde{h}+q \cdot s_{0}(n)+s_{2}(n)$ para todo $n \in \omega$. Os outros casos $(1 \in I$ e $2 \notin I, 1 \notin I$ e $2 \in I, 1,2 \notin I)$ são tratados de maneira análoga.

Proposição 7.1.5. Existe $\left\{F_{\xi}: 0<\xi<\mathfrak{c}\right\}$ uma indexação de $\mathcal{F}$ tal que $\cup_{n \in \omega} \operatorname{supp} f(n) \subset \xi \times \omega$, para toda $f \in F_{\xi}$ e todo $\left.\xi \in\right] 0, \mathfrak{c}[$.

Demonstração. Observamos, primeiramente, que $|\mathcal{F}|=\mathfrak{c}^{\omega}=\mathfrak{c}$. Portanto, podemos considerar $\left\{\tilde{F}_{\xi}: 0<\xi<\mathfrak{c}\right\}$ uma indexação de $\mathcal{F}$ tal que, para cada $F \in \mathcal{F}$, o conjunto

$$
A_{F}=\{\xi \in] 0, \mathfrak{c}\left[: F=\tilde{F}_{\xi}\right\}
$$

tenha cardinalidade $\mathfrak{c}$.

Fixemos $F \in \mathcal{F}$. Como $\cup_{f \in F} \cup_{n \in \omega} \operatorname{supp} f(n) \subset \mathfrak{c} \times \omega, \operatorname{cf}(\mathfrak{c})>\omega$ e $\left|\cup_{f \in F} \cup_{n \in \omega} \operatorname{supp} f(n)\right| \leq \omega$, existe $\alpha<\mathfrak{c}$ tal que $\cup_{f \in F} \cup_{n \in \omega} \operatorname{supp} f(n) \subset \alpha \times \omega$. Seja $\xi \in A_{F}$. Se $\xi<\alpha$, façamos $F_{\xi}=\{f\}$, onde $f(n)=(n+1) \cdot \chi_{(0,0)}$. Temos que $f$ é do tipo 2 e, portanto, $F_{\xi}$ é do tipo $A$. Em particular, $F_{\xi} \in \mathcal{F}$. Se $\xi \geq \alpha$, façamos $F_{\xi}=\tilde{F}_{\xi}$.

Vê-se facilmente que $\left\{F_{\xi}: 0<\xi<\mathfrak{c}\right\}$ é uma indexação de $\mathcal{F}$ tal que $\cup_{n \in \omega} \operatorname{supp} f(n) \subset \xi \times \omega$, para toda $f \in F_{\xi}$ e todo $\left.\xi \in\right] 0, \mathfrak{c}[$.

Se $\xi \in] 0, \mathfrak{c}\left[\right.$, denotaremos por $n\left(F_{\xi}\right)$ a cardinalidade de $F_{\xi}$ e escreveremos $F_{\xi}=$ 
$\left\{f_{\xi, 0}, \ldots, f_{\xi, n\left(F_{\xi}\right)-1}\right\}$. Se $F_{\xi}$ é do tipo $\mathrm{C}$, o limite da seqüência

$$
\left\{\frac{f_{\xi, 0}(n)\left(\theta_{n}, m_{n}\right)}{f_{\xi, 1}(n)\left(\theta_{n}, m_{n}\right)}: n \in \omega\right\}
$$

será denotado por $\zeta_{\xi}$.

Seja $\zeta \in \mathbb{R} \backslash \mathbb{Q}$. Do teorema de Kronecker segue que $\{(x+\mathbb{Z}, \zeta x+\mathbb{Z}): x \in \mathbb{R}\}$ é um subconjunto denso do toro $\mathbb{T}^{2}$. Portanto, para cada $\epsilon>0$, existe $l_{\zeta}(\epsilon)>0$ de modo que $\{(x+\mathbb{Z}, \zeta x+\mathbb{Z}): x \in I\}$ é $\epsilon$-denso no toro $\mathbb{T}^{2}$, para todo $I$ intervalo aberto de $\mathbb{R}$ de comprimento maior ou igual a $l_{\zeta}(\epsilon)$. Podemos supor que se $\epsilon_{1} \leq \epsilon_{2}$, então $l_{\zeta}\left(\epsilon_{1}\right) \geq l_{\zeta}\left(\epsilon_{2}\right)$.

\subsection{Construindo homomorfismos de grupos}

Sejam $\left\{p_{\xi}: 0<\xi<\mathfrak{c}\right\}$ uma família de ultrafiltros seletivos dois a dois incomparáveis e $\left\{F_{\xi}: 0<\xi<\mathfrak{c}\right\}$ uma indexação de $\mathcal{F}$ tal que $\cup_{n \in \omega} \operatorname{supp} f_{\xi, j}(n) \subset \xi \times \omega$ para todo $j<n\left(F_{\xi}\right)$ e todo $\xi \in] 0, \mathfrak{c}[$.

Proposição 7.2.1. Seja $J \in \mathbb{Q}^{(\mathfrak{c} \times \omega)} \backslash\{0\}$. Existe $E \in[\mathfrak{c}]^{\omega}$ de modo que supp $J \subset E \times \omega$ e tal que se $\xi \in E \backslash\{0\}$, então $\cup_{n \in \omega} \operatorname{supp} f_{\xi, j}(n) \subset E \times \omega$, para todo $j<n\left(F_{\xi}\right)$.

Demonstração. Façamos $E(0)=\omega$. Se $\xi \in] 0, \mathfrak{c}[$, definimos indutivamente

$$
E(\xi)=\{\xi\} \cup \bigcup_{\mu \in X_{\xi}} E(\mu)
$$

onde

$$
X_{\xi}=\pi_{1}\left[\cup_{j<n\left(F_{\xi}\right)} \cup_{n \in \omega} \operatorname{supp} f_{\xi, j}(n)\right]
$$

Seja

$$
E=\cup_{\zeta \in \pi_{1}[\operatorname{supp} J]} E(\zeta)
$$

Temos que supp $J \subset E \times \omega$, uma vez que $\zeta \in E(\zeta) \subset E$, qualquer que seja $\zeta \in \pi_{1}[\operatorname{supp} J]$. Além disso, um argumento indutivo garante que $E(\xi) \in[\mathfrak{c}]^{\omega}$, para todo $\xi<\mathfrak{c}$ e, portanto, $E \in[\mathfrak{c}]^{\omega}$. Por fim, se $\xi \in E \backslash\{0\}$, então existe $\zeta \in \pi_{1}[\operatorname{supp} J]$ tal que $\xi \in E(\zeta)$. Um outro argumento indutivo garante que se $\alpha \in E(\beta)$, então $E(\alpha) \subset E(\beta)$, quaisquer que sejam $\alpha, \beta<\mathfrak{c}$. Logo, $\pi_{1}\left[\cup_{n \in \omega} \operatorname{supp} f_{\xi, j}(n)\right] \subset E(\xi) \subset E(\zeta) \subset E$, para todo $j<n\left(F_{\xi}\right)$.

Lema 7.2.2. Sejam $J \in \mathbb{Q}^{(\mathfrak{c} \times \omega)} \backslash\{0\}$ e $E \in[\mathfrak{c}]^{\omega}$ de modo que $\operatorname{supp} J \subset E \times \omega$ e tal que se $\xi \in E \backslash\{0\}$, então $\cup_{n \in \omega} \operatorname{supp} f_{\xi, j}(n) \subset E \times \omega$, para todo $j<n\left(F_{\xi}\right)$. Existe $\left\{E_{k}: k \in \omega\right\}$ uma 
familia de subconjuntos finitos de $E,\left\{e_{k}: k \in \omega\right\} e\left\{b_{k}: k \in \omega\right\}$ seqüências estritamente crescentes de números naturais, $\left\{r_{k}: k \in \omega\right\}$ uma seqüência de números reais positivos $e$ $i: \omega \rightarrow E \backslash\{0\}$ tais que:

(i) $\operatorname{supp} J \subset E_{0} \times e_{0}$;

(ii) $E \times \omega=\cup_{k \in \omega}\left(E_{k} \times e_{k}\right)$;

(iii) $i(k) \in E_{k}$, para todo $k \in \omega$;

(iv) $e_{k}>n\left(F_{i(k)}\right)$, para todo $k \in \omega$;

(v) $\left(E_{k+1} \times e_{k+1}\right) \supset\left(E_{k} \times e_{k}\right) \cup \bigcup\left\{\operatorname{supp} f_{i(m), j}\left(b_{m}\right): j<n\left(F_{i(m)}\right), m \leq k\right\}$, para todo $k \in \omega$;

(vi) $\left\{b_{k}: k \in i^{-1}(\{\xi\})\right\} \in p_{\xi}$, para todo $\xi \in E \backslash\{0\}$;

(vii) Se $F_{i(k)}$ é do tipo A, então:

- $\operatorname{supp} f_{i(k), j}\left(b_{k}\right) \backslash\left(E_{k} \times e_{k}\right) \neq \emptyset$, se $f_{i(k), j}$ é do tipo 1 e $j<n\left(F_{i(k)}\right)$;

- $\left|a\left(f_{i(k), j}\left(b_{k}\right)\right)\right| \cdot r_{k}>4 \cdot d\left(F_{i(k)}\right)$, se $f_{i(k), j}$ é do tipo 2 e $j<n\left(F_{i(k)}\right)$;

(viii) Se $F_{i(k)}$ é do tipo B, então:

- $\operatorname{supp} f_{i(k), j}\left(b_{k}\right) \backslash\left(E_{k} \times e_{k}\right) \neq \emptyset$, se $\tilde{f}_{i(k), j}$ é do tipo 1 e $j<2$;

- $\left|a\left(f_{i(k), j}\left(b_{k}\right)\right)\right| \cdot r_{k}>4 \cdot d\left(F_{i(k)}\right)$, se $\tilde{f}_{i(k), j}$ é do tipo 2 e $j<2$;

- $\operatorname{supp} f_{i(k), j}\left(b_{k}\right) \backslash\left(E_{k} \times e_{k}\right) \neq \emptyset$, se $f_{i(k), j}$ é do tipo 1 e $2 \leq j<n\left(F_{i(k)}\right)$;

- $\left|a\left(f_{i(k), j}\left(b_{k}\right)\right)\right| \cdot r_{k}>4 \cdot d\left(F_{i(k)}\right)$, se $f_{i(k), j}$ é do tipo 2 e $2 \leq j<n\left(F_{i(k)}\right)$;

- $\left|a\left(f_{i(k), 1}\left(b_{k}\right),\left(\theta_{b_{k}}, m_{b_{k}}\right)\right)\right| \cdot r_{k}>4 \cdot\left|a\left(f_{i(k), 0}\left(b_{k}\right),\left(\theta_{b_{k}}, m_{b_{k}}\right)\right)\right| \cdot d\left(F_{i(k)}\right)$;

(ix) Se $F_{i(k)}$ é do tipo $C$, então:

- $\operatorname{supp} f_{i(k), j}\left(b_{k}\right) \backslash\left(E_{k} \times e_{k}\right) \neq \emptyset$, se $f_{i(k), j}$ é do tipo 1 e $2 \leq j<n\left(F_{i(k)}\right)$;

- $\left|a\left(f_{i(k), j}\left(b_{k}\right)\right)\right| \cdot r_{k}>4 \cdot d\left(F_{i(k)}\right)$, se $f_{i(k), j}$ é do tipo 2 e $2 \leq j<n\left(F_{i(k)}\right)$;

- $\left|a\left(f_{i(k), 1}\left(b_{k}\right),\left(\theta_{b_{k}}, m_{b_{k}}\right)\right)\right| \cdot r_{k}>4 \cdot l_{\zeta_{i(k)}}\left(\frac{r_{k}}{8}\right)$;

- $\left|\frac{a\left(f_{i(k), 0}\left(b_{k}\right),\left(\theta_{b_{k}}, m_{b_{k}}\right)\right)}{a\left(f_{i(k), 1}\left(b_{k}\right),\left(\theta_{b_{k}}, m_{b_{k}}\right)\right)} \cdot l_{\zeta_{i(k)}}\left(\frac{r_{k}}{8}\right)-\zeta_{i(k)} \cdot l_{\zeta_{i(k)}}\left(\frac{r_{k}}{8}\right)\right|<\frac{r_{k}}{8}$; 
(x) $r_{0}=\frac{1}{4 \cdot \sum_{(\xi, n) \in \operatorname{supp} J}|a(J,(\xi, n))|}$

(xi) $r_{k+1}=\frac{r_{k}}{2 \cdot \sum_{j<n\left(F_{i(k)}\right)} \sum_{(\xi, n) \in \operatorname{supp} f_{i(k), j}\left(b_{k}\right)}\left|a\left(f_{i(k), j}\left(b_{k}\right),(\xi, n)\right)\right|}$.

Demonstração. Consideremos $\left\{a_{n}: n \in \omega\right\}$ uma enumeração de $E \times \omega$. Seja

$$
G_{0}=\pi_{1}\left[\operatorname{supp} J \cup\left\{a_{0}\right\}\right]
$$

e tomemos $g_{0} \in \omega$ tal que $g_{0}>\max \pi_{2}\left[\operatorname{supp} J \cup\left\{a_{0}\right\}\right]$. Para cada $n \in \omega$, definimos

$$
G_{n+1}=\pi_{1}\left[\left\{a_{n+1}\right\} \cup \bigcup\left\{\operatorname{supp} f_{\xi, j}(m): m \leq n, \xi \in G_{n} \backslash\{0\}, j<n\left(F_{\xi}\right)\right\}\right] \cup G_{n}
$$

e tomemos $g_{n+1} \in \omega$ tal que $g_{n+1}>g_{n}$

$$
g_{n+1}>\max \left\{n\left(F_{\xi}\right): \xi \in G_{n+1} \backslash\{0\}\right\}
$$

e

$$
g_{n+1}>\max \pi_{2}\left[\left\{a_{n+1}\right\} \cup \bigcup\left\{\operatorname{supp} f_{\xi, j}(m): m \leq n, \xi \in G_{n} \backslash\{0\}, j<n\left(F_{\xi}\right)\right\}\right] .
$$

Temos que $\left\{G_{n}: n \in \omega\right\}$ é uma família de subconjuntos finitos de $E$ e $\left\{g_{n}: n \in \omega\right\}$ é uma seqüência estritamente crescente de números naturais tais que:

(1) $\operatorname{supp} J \subset G_{0} \times g_{0}$;

(2) $E \times \omega=\cup_{n \in \omega} G_{n} \times g_{n}$;

(3) $\left(G_{n+1} \times g_{n+1}\right) \supset\left(G_{n} \times g_{n}\right) \cup \bigcup\left\{\operatorname{supp} f_{\xi, j}(m): m \leq n, \xi \in G_{n} \backslash\{0\}, j<n\left(F_{\xi}\right)\right\}$.

Fixemos $\xi \in E \backslash\{0\}$ e $n \in \omega$. Suponhamos, primeiramente, que $F_{\xi}$ seja do tipo $A$. Se $j<n\left(F_{\xi}\right)$ e $f_{\xi, j}$ é do tipo 1 , definimos

$$
A_{n}^{\xi, j}=\left\{k \in \omega: \operatorname{supp} f_{\xi, j}(k) \backslash\left(G_{n} \times g_{n}\right) \neq \emptyset\right\} .
$$

Se $j<n\left(F_{\xi}\right)$ e $f_{\xi, j}$ é do tipo 2 , definimos

$$
A_{n}^{\xi, j}=\left\{k \in \omega:\left|a\left(f_{\xi, j}(k)\right)\right|>2^{n+4} \cdot d\left(F_{\xi}\right) \cdot X_{n-1}\right\}
$$


onde

$$
X_{p}=\sum_{(\mu, k) \in \operatorname{supp} J}|a(J,(\mu, k))| \cdot \prod_{\substack{l \leq p \\ \zeta \in G_{p} \backslash\{0\}}}\left(\sum_{j<n\left(F_{\zeta}\right)} \sum_{(\mu, k) \in \operatorname{supp} f_{\zeta, j}(l)}\left|a\left(f_{\zeta, j}(l),(\mu, k)\right)\right|\right)
$$

para cada $p \in \omega \mathrm{e}$

$$
X_{-1}=\sum_{(\mu, k) \in \operatorname{supp} J}|a(J,(\mu, k))| .
$$

Por fim, definimos

$$
A_{n}^{\xi}=\cap_{j<n\left(F_{\xi}\right)} A_{n}^{\xi, j}
$$

Suponhamos, agora, que $F_{\xi}$ seja do tipo $B$. Se $j<2$ e $\tilde{f}_{\xi, j}$ é do tipo 1 , definimos

$$
A_{n}^{\xi, j}=\left\{k \in \omega: \operatorname{supp} f_{\xi, j}(k) \backslash\left(G_{n} \times g_{n}\right) \neq \emptyset\right\} .
$$

Se $j<2$ e $\tilde{f}_{\xi, j}$ é do tipo 2 , definimos

$$
A_{n}^{\xi, j}=\left\{k \in \omega:\left|a\left(f_{\xi, j}(k)\right)\right|>2^{n+4} \cdot d\left(F_{\xi}\right) \cdot X_{n-1}\right\} .
$$

Se $2 \leq j<n\left(F_{\xi}\right)$ e $f_{\xi, j}$ é do tipo 1 , definimos

$$
A_{n}^{\xi, j}=\left\{k \in \omega: \operatorname{supp} f_{\xi, j}(k) \backslash\left(G_{n} \times g_{n}\right) \neq \emptyset\right\} .
$$

Se $2 \leq j<n\left(F_{\xi}\right)$ e $f_{\xi, j}$ é do tipo 2 , definimos

$$
A_{n}^{\xi, j}=\left\{k \in \omega:\left|a\left(f_{\xi, j}(k)\right)\right|>2^{n+4} \cdot d\left(F_{\xi}\right) \cdot X_{n-1}\right\} .
$$

Seja, também,

$$
B_{n}^{\xi}=\left\{k \in \omega:\left|a\left(f_{\xi, 1}(k),\left(\theta_{k}, m_{k}\right)\right)\right|>2^{n+4} \cdot d\left(F_{\xi}\right) \cdot\left|a\left(f_{\xi, 0}(k),\left(\theta_{k}, m_{k}\right)\right)\right| \cdot X_{n-1}\right\}
$$

Definimos

$$
A_{n}^{\xi}=\bigcap_{j<n\left(F_{\xi}\right)} A_{n}^{\xi, j} \cap B_{n}^{\xi}
$$


Finalmente, suponhamos que $F_{\xi}$ seja do tipo $C$. Se $2 \leq j<n\left(F_{\xi}\right)$ e $f_{\xi, j}$ é do tipo 1 , definimos

$$
A_{n}^{\xi, j}=\left\{k \in \omega: \operatorname{supp} f_{\xi, j}(k) \backslash\left(G_{n} \times g_{n}\right) \neq \emptyset\right\} .
$$

Se $2 \leq j<n\left(F_{\xi}\right)$ e $f_{\xi, j}$ é do tipo 2 , definimos

$$
A_{n}^{\xi, j}=\left\{k \in \omega:\left|a\left(f_{\xi, j}(k)\right)\right|>2^{n+4} \cdot d\left(F_{\xi}\right) \cdot X_{n-1}\right\} .
$$

Façamos

$$
A_{n}^{\xi, 1}=\left\{k \in \omega:\left|a\left(f_{\xi, 1}(k),\left(\theta_{k}, m_{k}\right)\right)\right|>2^{n+4} \cdot l_{n, \xi} \cdot X_{n-1}\right\}
$$

e

$$
C_{\xi}^{n}=\left\{k \in \omega:\left|\frac{a\left(f_{\xi, 0}(k),\left(\theta_{k}, m_{k}\right)\right)}{a\left(f_{\xi, 1}(k),\left(\theta_{k}, m_{k}\right)\right)} \cdot l_{n, \xi}-\zeta_{\xi} \cdot l_{n, \xi}\right| \cdot 2^{n+5} \cdot X_{n-1}<1\right\}
$$

onde

$$
l_{n, \xi}=l_{\zeta_{\xi}}\left(\frac{1}{2^{n+5} \cdot X_{n-1}}\right)
$$

Definimos

$$
A_{n}^{\xi}=\bigcap_{1 \leq j<n\left(F_{\xi}\right)} A_{n}^{\xi, j} \cap C_{n}^{\xi}
$$

Observamos que $A_{n}^{\xi}$ é um subconjunto cofinito de $\omega$, quaisquer que sejam $n \in \omega$ e $\xi \in E \backslash\{0\} .{ }^{2}$ Logo, para cada $\xi \in E \backslash\{0\}$, temos que $\left\{A_{n}^{\xi}: n \in \omega\right\} \subset p_{\xi}$, uma vez que $p_{\xi}$ é um ultrafiltro livre sobre $\omega$. Da seletividade de $p_{\xi}$ decorre que existe uma seqüência $\left\{a_{n}^{\xi}: n \in \omega\right\} \in p_{\xi}$ tal que $a_{n}^{\xi} \in A_{n}^{\xi}$ e $n<a_{n}^{\xi}$, para cada $n \in \omega$.

Do lema 1.5.4 segue que existe $\left\{I_{\xi}: \xi \in E \backslash\{0\}\right\}$ uma família de subconjuntos de $\omega$ dois a dois disjuntos tal que:

(a) $\left\{a_{n}^{\xi}: n \in I_{\xi}\right\} \in p_{\xi}$, para cada $\xi \in E \backslash\{0\}$;

(b) $\left\{\left[n, a_{n}^{\xi}\right]: n \in I_{\xi}, \xi \in E \backslash\{0\}\right\}$ é uma família de intervalos de $\omega$ dois a dois disjuntos.

Para cada $\xi \in E \backslash\{0\}$, seja

(c) $N_{\xi}=\min \left\{n \in \omega: \xi \in G_{n}\right\}$.

\footnotetext{
${ }^{2}$ Se $F_{\xi}$ é do tipo $B$, então $B_{n}^{\xi}$ é um subconjunto cofinito de $\omega$ já que $\frac{f_{\xi, 0}(k)\left(\left(\theta_{k}, m_{k}\right)\right)}{f_{\xi, 1}(k)\left(\left(\theta_{k}, m_{k}\right)\right)} \rightarrow 0$. Se $F_{\xi}$ é do tipo $C$, então $A_{n}^{\xi, 1}$ e $C_{n}^{\xi}$ são subconjuntos cofinitos de $\omega$, pois $\frac{f_{\xi, 0}(k)\left(\left(\theta_{k}, m_{k}\right)\right)}{f_{\xi, 1}(k)\left(\left(\theta_{k}, m_{k}\right)\right)} \rightarrow \zeta_{\xi}$ e $\zeta_{\xi} \in \mathbb{R} \backslash \mathbb{Q}$.
} 
Podemos supor, sem perda de generalidade, que

(d) $N_{\xi}<n$, para todo $n \in I_{\xi}$.

Consideremos $\left\{n_{k}: k \in \omega\right\}$ uma enumeração estritamente crescente de $\dot{\cup}_{\xi \in E \backslash\{0\}} I_{\xi}$. Seja

$$
\begin{aligned}
i: \omega & \rightarrow E \backslash\{0\} \\
k & \mapsto i(k)
\end{aligned}
$$

onde $i(k)$ é o único elemento de $E \backslash\{0\}$ tal que $n_{k} \in I_{i(k)}$.

Para cada $k \in \omega$, façamos $b_{k}=a_{n_{k}}^{i(k)}, E_{k}=G_{n_{k}}, e_{k}=g_{n_{k}}$ e definamos $r_{k}$ segundo os ítens (x) e (xi) do enunciado deste lema. Resta mostrar que as condições (i)-(ix) estão satisfeitas.

(i) Como $0 \leq n_{0}$, temos que $E_{0} \times e_{0}=G_{n_{0}} \times g_{n_{0}} \supset G_{0} \times g_{0} \supset \operatorname{supp} J$.

(ii) $\cup_{k \in \omega}\left(E_{k} \times e_{k}\right)=\cup_{k \in \omega}\left(G_{n_{k}} \times g_{n_{k}}\right)=E \times \omega$.

(iii) Seja $k \in \omega$. Temos que $n_{k} \in I_{i(k)}$. De (d) segue que $N_{i(k)}<n_{k}$. De (c) e (3) vem que $i(k) \in G_{N_{i(k)}} \subset G_{n_{k}}=E_{k}$.

(iv) $e_{k}=g_{n_{k}}>\max \left\{n\left(F_{\xi}\right): \xi \in G_{n_{k}} \backslash\{0\}\right\}$. Como $i(k) \in G_{n_{k}} \backslash\{0\}$, temos que $e_{k}>n\left(F_{i(k)}\right)$.

(v) De (3) vem que $E_{k+1} \times e_{k+1} \supset E_{k} \times e_{k}$, pois $\left\{n_{k}: k \in \omega\right\}$ é uma enumeração estritamente crescente de $\cup_{\xi \in E \backslash\{0\}} I_{\xi}$. Resta mostrar que $\operatorname{supp} f_{i(m), j}\left(b_{m}\right) \subset E_{k+1} \times e_{k+1}$, para cada $m \leq k$ e cada $j<n\left(F_{i(m)}\right)$. De $(3)$ decorre que

$E_{k+1} \times e_{k+1}=G_{n_{k+1}} \times g_{n_{k+1}} \supset \bigcup\left\{\operatorname{supp} f_{\xi, j}(m): m \leq n_{k+1}-1, \xi \in G_{n_{k+1}-1} \backslash\{0\}, j<n\left(F_{\xi}\right)\right\}$.

Por (iii), $i(m) \in E_{m} \subset E_{k}=G_{n_{k}} \subset G_{n_{k+1}-1}$, para todo $m \leq k$. Além disso, $i(m) \neq 0$. Por (b), $b_{m}=a_{n_{m}}^{i(m)} \leq n_{k+1}-1$, para todo $m \leq k$. Logo, supp $f_{i(m), j}\left(b_{m}\right) \subset E_{k+1} \times e_{k+1}$, para todo $j<n\left(F_{i(m)}\right)$.

(vi) Seja $\xi \in E \backslash\{0\}$. Temos que $\left\{b_{k}: k \in i^{-1}(\{\xi\})\right\}=\left\{a_{n_{k}}^{i(k)}: k \in i^{-1}(\{\xi\})\right\}=\left\{a_{n}^{\xi}: n \in I_{\xi}\right\} \in$ $p_{\xi}$, por $(\mathrm{a})$.

(vii) Suponhamos que $F_{i(k)}$ seja do tipo A. Temos que $b_{k}=a_{n_{k}}^{i(k)} \in A_{n_{k}}^{i(k)}$. Se $f_{i(k), j}$ é do tipo 1 e $j<n\left(F_{i(k)}\right)$, então

$$
\operatorname{supp} f_{i(k), j}\left(b_{k}\right) \backslash\left(E_{k} \times e_{k}\right)=\operatorname{supp} f_{i(k), j}\left(b_{k}\right) \backslash\left(G_{n_{k}} \times g_{n_{k}}\right) \neq \emptyset \text {. }
$$


Se $f_{i(k), j}$ é do tipo 2 e $j<n\left(F_{i(k)}\right)$, então

$$
\left|a\left(f_{i(k), j}\left(b_{k}\right)\right)\right|>2^{n_{k}+4} \cdot d\left(F_{i(k)}\right) \cdot X_{n_{k}-1}
$$

onde

$$
X_{n_{k}-1}=\sum_{(\theta, m) \in \operatorname{supp} J}|a(J,(\theta, m))| \cdot \prod_{\substack{l \leq n_{k}-1 \\ \zeta \in G_{n_{k}}-1 \backslash\{0\}}}\left(\sum_{j<n\left(F_{\zeta}\right)} \sum_{(\theta, m) \in \operatorname{supp} f_{\zeta, j}(l)}\left|a\left(f_{\zeta, j}(l),(\theta, m)\right)\right|\right) .
$$

Observamos que se $m<k$, então $n_{m} \leq n_{k-1}$ e, portanto, $i(m) \in E_{m}=G_{n_{m}} \subset G_{n_{k-1}} \subset$ $G_{n_{k}-1}$. Note que $i(m) \neq 0$. Como $b_{m}=a_{n_{m}}^{i(m)}$ e $\left[n_{m}, a_{n_{m}}^{i(m)}\right] \cap\left[n_{k}, a_{n_{k}}^{i(k)}\right]=\emptyset$, temos que $b_{m}<n_{k}$ e, portanto, $b_{m} \leq n_{k}-1$. Logo,

$$
\left|a\left(f_{i(k), j}\left(b_{k}\right)\right)\right| \cdot r_{k}>4 \cdot d\left(F_{i(k)}\right) .
$$

(viii) Suponhamos que $F_{i(k)}$ seja do tipo B. Os argumentos utilizados em (vii) nos permitem mostrar que:

- $\operatorname{supp} f_{i(k), j}\left(b_{k}\right) \backslash\left(E_{k} \times e_{k}\right) \neq \emptyset$, se $\tilde{f}_{i(k), j}$ é do tipo 1 e $j<2$;

- $\left|a\left(f_{i(k), j}\left(b_{k}\right)\right)\right| \cdot r_{k}>4 \cdot d\left(F_{i(k)}\right)$, se $\tilde{f}_{i(k), j}$ é do tipo 2 e $j<2$;

- $\operatorname{supp} f_{i(k), j}\left(b_{k}\right) \backslash\left(E_{k} \times e_{k}\right) \neq \emptyset$, se $f_{i(k), j}$ é do tipo 1 e $2 \leq j<n\left(F_{i(k)}\right)$;

- $\left|a\left(f_{i(k), j}\left(b_{k}\right)\right)\right| \cdot r_{k}>4 \cdot d\left(F_{i(k)}\right)$, se $f_{i(k), j}$ é do tipo 2 e $2 \leq j<n\left(F_{i(k)}\right)$.

Temos que $b_{k}=a_{n_{k}}^{i(k)} \in A_{n_{k}}^{i(k)}$. Da definição de $B_{n_{k}}^{i(k)}$ segue que

$$
\left|a\left(f_{i(k), 1}\left(b_{k}\right),\left(\theta_{b_{k}}, m_{b_{k}}\right)\right)\right|>2^{n_{k}+4} \cdot d\left(F_{i(k)}\right) \cdot\left|a\left(f_{i(k), 0}\left(b_{k}\right),\left(\theta_{b_{k}}, m_{b_{k}}\right)\right)\right| \cdot X_{n_{k}-1}
$$

Observamos que se $m<k$, então $n_{m} \leq n_{k-1}$ e, portanto, $i(m) \in E_{m}=G_{n_{m}} \subset G_{n_{k-1}} \subset$ $G_{n_{k}-1}$. Note que $i(m) \neq 0$. Como $b_{m}=a_{n_{m}}^{i(m)}$ e $\left[n_{m}, a_{n_{m}}^{i(m)}\right] \cap\left[n_{k}, a_{n_{k}}^{i(k)}\right]=\emptyset$, temos que $b_{m}<n_{k}$ e, portanto, $b_{m} \leq n_{k}-1$. Logo,

$$
\left|a\left(f_{i(k), 1}\left(b_{k}\right),\left(\theta_{b_{k}}, m_{b_{k}}\right)\right)\right| \cdot r_{k}>4 \cdot\left|a\left(f_{i(k), 0}\left(b_{k}\right),\left(\theta_{b_{k}}, m_{b_{k}}\right)\right)\right| \cdot d\left(F_{i(k)}\right) .
$$

(ix) Suponhamos que $F_{i(k)}$ seja do tipo C. Os argumentos utilizados em (vii) nos permitem mostrar que: 
- $\operatorname{supp} f_{i(k), j}\left(b_{k}\right) \backslash\left(E_{k} \times e_{k}\right) \neq \emptyset$, se $f_{i(k), j}$ é do tipo 1 e $2 \leq j<n\left(F_{i(k)}\right)$;

- $\left|a\left(f_{i(k), j}\left(b_{k}\right)\right)\right| \cdot r_{k}>4 \cdot d\left(F_{i(k)}\right)$, se $f_{i(k), j}$ é do tipo 2 e $2 \leq j<n\left(F_{i(k)}\right)$.

Temos que $b_{k}=a_{n_{k}}^{i(k)} \in A_{n_{k}}^{i(k)}$. Da definição de $A_{n_{k}}^{i(k), 1}$ segue que

$$
\left|a\left(f_{i(k), 1}\left(b_{k}\right),\left(\theta_{b_{k}}, m_{b_{k}}\right)\right)\right|>2^{n_{k}+4} \cdot l_{n_{k}, i(k)} \cdot X_{n_{k}-1}
$$

onde

$$
l_{n_{k}, i(k)}=l_{\zeta_{i(k)}}\left(\frac{1}{2^{n_{k}+5} \cdot X_{n_{k}-1}}\right) .
$$

Como

$$
\frac{1}{2^{n_{k}+5} \cdot X_{n_{k}-1}} \leq \frac{r_{k}}{8}
$$

temos que

$$
l_{\zeta_{i(k)}}\left(\frac{r_{k}}{8}\right) \leq l_{n_{k}, i(k)}
$$

Portanto,

$$
\left|a\left(f_{i(k), 1}\left(b_{k}\right),\left(\theta_{b_{k}}, m_{b_{k}}\right)\right)\right| \cdot r_{k}>4 \cdot l_{n_{k}, i(k)} \geq 4 \cdot l_{\zeta_{i(k)}}\left(\frac{r_{k}}{8}\right) .
$$

Da definição de $C_{n_{k}}^{i(k)}$ segue que

$$
\left|\frac{a\left(f_{i(k), 0}\left(b_{k}\right),\left(\theta_{b_{k}}, m_{b_{k}}\right)\right)}{a\left(f_{i(k), 1}\left(b_{k}\right),\left(\theta_{b_{k}}, m_{b_{k}}\right)\right)} \cdot l_{n_{k}, i(k)}-\zeta_{i(k)} \cdot l_{n_{k}, i(k)}\right| \cdot 2^{n_{k}+5} \cdot X_{n_{k}-1}<1
$$

e, portanto,

$$
\left|\frac{a\left(f_{i(k), 0}\left(b_{k}\right),\left(\theta_{b_{k}}, m_{b_{k}}\right)\right)}{a\left(f_{i(k), 1}\left(b_{k}\right),\left(\theta_{b_{k}}, m_{b_{k}}\right)\right)} \cdot l_{\zeta_{i(k)}}\left(\frac{r_{k}}{8}\right)-\zeta_{i(k)} \cdot l_{\zeta_{i(k)}}\left(\frac{r_{k}}{8}\right)\right|<\frac{r_{k}}{8} .
$$

Lema 7.2.3. Sejam $J \in \mathbb{Q}^{(\mathfrak{c} \times \omega)} \backslash\{0\}$ e $E \in[\mathfrak{c}]^{\omega}$ de modo que $\operatorname{supp} J \subset E \times \omega$ e tal que se $\xi \in$ $E \backslash\{0\}$, então $\cup_{n \in \omega} \operatorname{supp} f_{\xi, j}(n) \subset E \times \omega$, para todo $j<n\left(F_{\xi}\right)$. Existe $\phi_{J} \uparrow_{\mathbb{Q}^{(E \times \omega)}}: \mathbb{Q}^{(E \times \omega)} \rightarrow \mathbb{T}$ um homomorfismo de grupos com as seguintes propriedades:

(i) $\phi_{J} \uparrow_{\mathbb{Q}^{(E \times \omega)}}(J) \neq 0+\mathbb{Z}$;

(ii) Se $\xi \in E \backslash\{0\}$, então $\phi_{J} \uparrow_{\mathbb{Q}^{(E \times \omega)}}\left(\chi_{(\xi, j)}\right)=p_{\xi}-\lim \left\{\phi_{J} \Upsilon_{\mathbb{Q}^{(E \times \omega)}}\left(f_{\xi, j}(n)\right): n \in \omega\right\}$, para cada $j<n\left(F_{\xi}\right){ }^{3}$

\footnotetext{
${ }^{3}$ Estamos considerando $\mathbb{Q}^{(E \times \omega)} \subset \mathbb{Q}^{(\mathfrak{c} \times \omega)}$.
} 
Demonstração. Consideremos $\left\{E_{m}: m \in \omega\right\},\left\{e_{m}: m \in \omega\right\},\left\{b_{m}: m \in \omega\right\},\left\{r_{m}: m \in \omega\right\}$ e $i: \omega \rightarrow E \backslash\{0\}$ de acordo com o lema 7.2.2. A fim de exibirmos um homomorfismo de grupos $\phi_{J} \uparrow_{\mathbb{Q}^{(E \times \omega)}}$ satisfazendo as condições (i) e (ii) deste lema, construiremos indutivamente aplicações auxiliares $\psi_{m}: E \times \omega \rightarrow \mathcal{B}$ e $\psi_{m}^{*}: E_{m} \times e_{m} \rightarrow \mathcal{B}$, para cada $m \in \omega$.

Para cada $(\xi, n) \in E_{0} \times e_{0}$, tomemos $y_{(\xi, n)} \in \mathbb{R}$ tal que

$$
\sum_{(\xi, n) \in \operatorname{supp} J} J(\xi, n) \cdot y_{(\xi, n)}=\frac{1}{2}
$$

e façamos

$$
x_{(\xi, n)}=\frac{1}{d(J)} \cdot y_{(\xi, n)}+\mathbb{Z}
$$

Temos que

$$
\sum_{(\xi, n) \in \operatorname{supp} J} a(J,(\xi, n)) \cdot x_{(\xi, n)}=\frac{1}{2}+\mathbb{Z} .
$$

Seja $\psi_{0}^{*}(\xi, n)$ o arco aberto de $\mathbb{T}$ centrado em $x_{(\xi, n)}$ com diâmetro

$$
\delta\left(\psi_{0}^{*}(\xi, n)\right)=\frac{r_{0}}{d(J)}
$$

Seja também $\psi_{0}(\xi, n)=d(J) \cdot \psi_{0}^{*}(\xi, n)$. Claramente, $\delta\left(\psi_{0}(\xi, n)\right)=r_{0}$. Uma vez que

$$
\frac{1}{2}+\mathbb{Z} \in \sum_{(\xi, n) \in \operatorname{supp} J} a(J,(\xi, n)) \cdot \psi_{0}^{*}(\xi, n)
$$

e

$$
\delta\left(\sum_{(\xi, n) \in \operatorname{supp} J} a(J,(\xi, n)) \cdot \psi_{0}^{*}(\xi, n)\right) \leq \sum_{(\xi, n) \in \operatorname{supp} J}|a(J,(\xi, n))| \cdot \delta\left(\psi_{0}^{*}(\xi, n)\right) \leq \frac{1}{4}
$$

concluímos que

$$
0+\mathbb{Z} \notin \sum_{(\xi, n) \in \operatorname{supp} J} a(J,(\xi, n)) \cdot \psi_{0}^{*}(\xi, n) .
$$

Por fim, se $(\xi, n) \in(E \times \omega) \backslash\left(E_{0} \times e_{0}\right)$, façamos $\psi_{0}(\xi, n)=\mathbb{T}$.

Seja $m \in \omega$. Suponhamos definidas $\psi_{m}: E \times \omega \rightarrow \mathcal{B}$ e $\psi_{m}^{*}: E_{m} \times e_{m} \rightarrow \mathcal{B}$. Vamos construir $\psi_{m+1}: E \times \omega \rightarrow \mathcal{B}$ e $\psi_{m+1}^{*}: E_{m+1} \times e_{m+1} \rightarrow \mathcal{B}$ com as seguintes propriedades:

(1) Se $(\xi, n) \in(E \times \omega) \backslash\left(E_{m+1} \times e_{m+1}\right)$, então $\psi_{m+1}(\xi, n)=\mathbb{T}$;

(2) Se $(\xi, n) \in\left(E_{m+1} \times e_{m+1}\right) \backslash\left(E_{m} \times e_{m}\right)$, então $\psi_{m+1}^{*}(\xi, n)$ é tal que: 
(a) $b(J) \cdot \prod_{k<m+1} d\left(F_{i(k)}\right) \cdot \overline{\psi_{m+1}^{*}(\xi, n)} \subset \psi_{m}(\xi, n)$;

(b) $\delta\left(\psi_{m+1}^{*}(\xi, n)\right)=\frac{r_{m+1}}{b(J) \cdot \prod_{k<m+1} d\left(F_{i(k)}\right)}$.

Neste caso, façamos

$$
\psi_{m+1}(\xi, n)=b(J) \cdot \prod_{k<m+1} d\left(F_{i(k)}\right) \cdot \psi_{m+1}^{*}(\xi, n) .
$$

(3) Se $(\xi, n) \in E_{m} \times e_{m}$, então $\psi_{m+1}^{*}(\xi, n)$ é tal que:

(a) $d\left(F_{i(m)}\right) \cdot \overline{\psi_{m+1}^{*}(\xi, n)} \subset \psi_{m}^{*}(\xi, n)$;

(b) $\delta\left(\psi_{m+1}^{*}(\xi, n)\right)=\frac{r_{m+1}}{b(J) \cdot \prod_{k<m+1} d\left(F_{i(k)}\right)}$.

Neste caso, façamos

$$
\psi_{m+1}(\xi, n)=b(J) \cdot \prod_{k<m+1} d\left(F_{i(k)}\right) \cdot \psi_{m+1}^{*}(\xi, n) .
$$

(4) Para cada $j<n\left(F_{i(m)}\right)$,

$$
\psi_{m}(i(m), j) \cap\left(\sum_{(\xi, n) \in \operatorname{supp} f_{i(m), j}\left(b_{m}\right)} a\left(f_{i(m), j}\left(b_{m}\right),(\xi, n)\right) \cdot b(J) \cdot \prod_{k<m} d\left(F_{i(k)}\right) \cdot \psi_{m+1}^{*}(\xi, n)\right) \neq \emptyset .
$$

Se $(\xi, n) \in(E \times \omega) \backslash\left(E_{m+1} \times e_{m+1}\right)$, façamos $\psi_{m+1}(\xi, n)=\mathbb{T}$. Se $(\xi, n) \in\left[\left(E_{m+1} \times e_{m+1}\right) \backslash\right.$ $\left.\left(E_{m} \times e_{m}\right)\right] \backslash \cup_{j<n\left(F_{i(m)}\right)} \operatorname{supp} f_{i(m), j}\left(b_{m}\right)$, então $\psi_{m}(\xi, n)=\mathbb{T}$. Definimos $\psi_{m+1}^{*}(\xi, n)$ como sendo um elemento de $\mathcal{B}$ com diâmetro

$$
\delta\left(\psi_{m+1}^{*}(\xi, n)\right)=\frac{r_{m+1}}{b(J) \cdot \prod_{k<m+1} d\left(F_{i(k)}\right)}
$$

e façamos

$$
\psi_{m+1}(\xi, n)=b(J) \cdot \prod_{k<m+1} d\left(F_{i(k)}\right) \cdot \psi_{m+1}^{*}(\xi, n) .
$$

Se $(\xi, n) \in\left(E_{m} \times e_{m}\right) \backslash \cup_{j<n\left(F_{i(m)}\right)} \operatorname{supp} f_{i(m), j}\left(b_{m}\right)$, fixemos uma raiz $d\left(F_{i(m)}\right)$-ésima do ponto médio de $\psi_{m}^{*}(\xi, n)$. Definimos $\psi_{m+1}^{*}(\xi, n)$ como sendo o arco aberto de $\mathbb{T}$ centrado na raiz em questão com diâmetro

$$
\delta\left(\psi_{m+1}^{*}(\xi, n)\right)=\frac{r_{m+1}}{b(J) \cdot \prod_{k<m+1} d\left(F_{i(k)}\right)}
$$


e façamos

$$
\psi_{m+1}(\xi, n)=b(J) \cdot \prod_{k<m+1} d\left(F_{i(k)}\right) \cdot \psi_{m+1}^{*}(\xi, n) .
$$

Vamos, agora, definir $\psi_{m+1}^{*}(\xi, n)$ e $\psi_{m+1}(\xi, n)$ para $(\xi, n) \in \cup_{j<n\left(F_{i(m)}\right)} \operatorname{supp} f_{i(m), j}\left(b_{m}\right)$.

Caso 1: $F_{i(m)}$ é do tipo A.

Seja $j<n\left(F_{i(m)}\right)$. Fixemos $(\mu, k) \in \operatorname{supp} f_{i(m), j}\left(b_{m}\right)$ tal que:

- $(\mu, k) \notin E_{m} \times e_{m}$, se $f_{i(m), j}$ é do tipo 1 ;

- $\left|a\left(f_{i(m), j}\left(b_{m}\right),(\mu, k)\right)\right| \cdot r_{m}>4 \cdot d\left(F_{i(m)}\right)$, se $f_{i(m), j}$ é do tipo 2 .

Para cada $(\xi, n) \in \operatorname{supp} f_{i(m), j}\left(b_{m}\right) \backslash\{(\mu, k)\}$, fixemos $z_{(\xi, n)} \in \mathbb{T}$ da seguinte maneira: se $(\xi, n) \in E_{m} \times e_{m}$, então $z_{(\xi, n)}$ é uma raiz $d\left(F_{i(m)}\right)$-ésima do ponto médio de $\psi_{m}^{*}(\mu, k)$; se $(\xi, n) \notin E_{m} \times e_{m}$, então $z_{(\xi, n)}$ é escolhido arbitrariamente. Seja

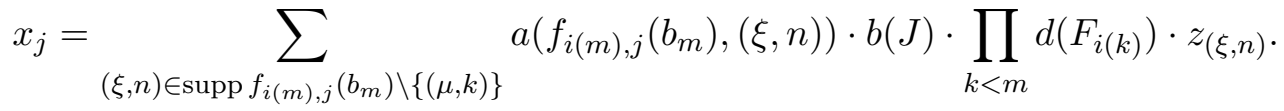

Se $f_{i(m), j}$ é do tipo 1 , tomemos $z_{(\mu, k)} \in \mathbb{T}$ tal que

$$
a\left(f_{i(m), j}\left(b_{m}\right),(\mu, k)\right) \cdot b(J) \cdot \prod_{k<m} d\left(F_{i(k)}\right) \cdot z_{(\mu, k)}+x_{j} \in \psi_{m}(i(m), j)
$$

Se $f_{i(m), j}$ é do tipo 2, podemos supor que $(\mu, k) \in E_{m} \times e_{m}$ pois, do contrário, os argumentos utilizados acima podem ser repetidos aqui. Sejam $\tilde{z}_{(\mu, k)}$ uma raiz $d\left(F_{i(m)}\right)$-ésima do ponto médio de $\psi_{m}^{*}(\mu, k)$ e $A$ o arco aberto de $\mathbb{T}$ centrado em $\tilde{z}_{(\mu, k)}$ com diâmetro

$$
\delta(A)=\frac{r_{m}}{4 \cdot b(J) \cdot \prod_{k<m+1} d\left(F_{i(k)}\right)} .
$$

Observamos que

$$
a\left(f_{i(m), j}\left(b_{m}\right),(\mu, k)\right) \cdot b(J) \cdot \prod_{k<m} d\left(F_{i(k)}\right) \cdot A=\mathbb{T}
$$

e, portanto, existe $z_{(\mu, k)} \in A$ tal que

$$
a\left(f_{i(m), j}\left(b_{m}\right),(\mu, k)\right) \cdot b(J) \cdot \prod_{k<m} d\left(F_{i(k)}\right) \cdot z_{(\mu, k)}+x_{j} \in \psi_{m}(i(m), j) .
$$


Para cada $(\xi, n) \in \operatorname{supp} f_{i(m), j}\left(b_{m}\right)$, seja $\psi_{m+1}^{*}(\xi, n)$ o arco aberto de $\mathbb{T}$ centrado em $z_{(\xi, n)}$ com diâmetro

$$
\delta\left(\psi_{m+1}^{*}(\xi, n)\right)=\frac{r_{m+1}}{b(J) \cdot \prod_{k<m+1} d\left(F_{i(k)}\right)}
$$

e façamos

$$
\psi_{m+1}(\xi, n)=b(J) \cdot \prod_{k<m+1} d\left(F_{i(k)}\right) \cdot \psi_{m+1}^{*}(\xi, n) .
$$

As condições (1), (2), (3) e (4) estão satisfeitas.

Caso 2: $F_{i(m)}$ é do tipo B.

Se $(\xi, n) \in \cup_{2 \leq j<n\left(F_{i(m)}\right)} \operatorname{supp} f_{i(m), j}\left(b_{m}\right)$, procedemos como no caso 1 para definir $\psi_{m+1}^{*}(\xi, n)$ e $\psi_{m+1}(\xi, n)$. Fixemos $(\mu, k) \in \operatorname{supp} f_{i(m), 0}\left(b_{m}\right)$ tal que

$$
\left|a\left(f_{i(m), 1}\left(b_{m}\right),(\mu, k)\right)\right| \cdot r_{m}>4 \cdot\left|a\left(f_{i(m), 0}\left(b_{m}\right),(\mu, k)\right)\right| \cdot d\left(F_{i(m)}\right)
$$

e fixemos $(\nu, l) \in \operatorname{supp} f_{i(m), 0}\left(b_{m}\right)$ tal que:

- $(\nu, l) \notin E_{m} \times e_{m}$, se $\tilde{f}_{i(m), 0}$ é do tipo 1 ;

- $\left|a\left(f_{i(m), 0}\left(b_{m}\right),(\nu, l)\right)\right| \cdot r_{m}>4 \cdot d\left(F_{i(m)}\right)$, se $\tilde{f}_{i(m), 0}$ é do tipo 2 .

Para cada $(\xi, n) \in \operatorname{supp} f_{i(m), 1}\left(b_{m}\right) \backslash\{(\mu, k),(\nu, l)\}$, definimos $z_{(\xi, n)}$ como no caso 1.

Se $(\mu, k)=(\nu, l)$, sejam $x_{0}$ e $x_{1}$ também como no caso 1 . Seja $\tilde{z}_{(\mu, k)}$ uma raiz $a\left(f_{i(m), 0}\left(b_{m}\right),(\mu, k)\right) \cdot b(J) \cdot \prod_{k<m} d\left(F_{i(k)}\right)$-ésima do ponto médio de $\psi_{m}(i(m), 0)-x_{0}$. Se $(\mu, k) \in E_{m} \times e_{m}$, pedimos também que $d\left(F_{i(m)}\right) \cdot \tilde{z}_{(\mu, k)} \in \tilde{\psi}_{m}^{*}(\mu, k)$, onde $\tilde{\psi}_{m}^{*}(\mu, k)$ é o arco aberto de $\mathbb{T}$ centrado no ponto médio de $\psi_{m}^{*}(\mu, k)$ com diâmetro

$$
\delta\left(\tilde{\psi}_{m}^{*}(\mu, k)\right)=\frac{\delta\left(\psi_{m}^{*}(\mu, k)\right)}{4} \cdot 4
$$

Seja $I$ o arco aberto de $\mathbb{T}$ centrado em $\tilde{z}_{(\mu, k)}$ com diâmetro

$$
\delta(I)=\frac{r_{m}}{4 \cdot\left|a\left(f_{i(m), 0}\left(b_{m}\right),(\mu, k)\right)\right| \cdot b(J) \cdot \prod_{k<m+1} d\left(F_{i(k)}\right)} .
$$

\footnotetext{
${ }^{4}$ Isto pode ser feito como no caso 1.
} 
Existe $z_{(\mu, k)} \in I$ tal que

$$
a\left(f_{i(m), 1}\left(b_{m}\right),(\mu, k)\right) \cdot b(J) \cdot \prod_{k<m} d\left(F_{i(k)}\right) \cdot z_{(\mu, k)}+x_{1} \in \psi_{m}(i(m), 1)
$$

e

$$
a\left(f_{i(m), 0}\left(b_{m}\right),(\mu, k)\right) \cdot b(J) \cdot \prod_{k<m} d\left(F_{i(k)}\right) \cdot z_{(\mu, k)}+x_{0} \in \psi_{m}(i(m), 0) .
$$

Para cada $(\xi, n) \in \operatorname{supp} f_{i(m), 1}\left(b_{m}\right)$, seja $\psi_{m+1}^{*}(\xi, n)$ o arco aberto de $\mathbb{T}$ centrado em $z_{(\xi, n)}$ com diâmetro

$$
\delta\left(\psi_{m+1}^{*}(\xi, n)\right)=\frac{r_{m+1}}{b(J) \cdot \prod_{k<m+1} d\left(F_{i(k)}\right)}
$$

e façamos

$$
\psi_{m+1}(\xi, n)=b(J) \cdot \prod_{k<m+1} d\left(F_{i(k)}\right) \cdot \psi_{m+1}^{*}(\xi, n) .
$$

As condições (1), (2), (3) e (4) estão satisfeitas.

Suponhamos, agora, que $(\mu, k) \neq(\nu, l)$. Se $(\mu, k) \in E_{m} \times e_{m}$, seja $\tilde{z}_{(\mu, k)}$ uma raiz $d\left(F_{i(m)}\right)$ ésima do ponto médio de $\psi_{m}^{*}(\mu, k)$. Se $(\mu, k) \notin E_{m} \times e_{m}$, seja $\tilde{z}_{(\mu, k)}$ um elemento arbitrário de $\mathbb{T}$. Seja $z_{(\nu, l)}$ uma raiz $a\left(f_{i(m), 0}\left(b_{m}\right),(\nu, l)\right) \cdot b(J) \cdot \prod_{k<m} d\left(F_{i(k)}\right)$-ésima do ponto médio de $\psi_{m}(i(m), 0)-\tilde{x}_{0}-x$, onde

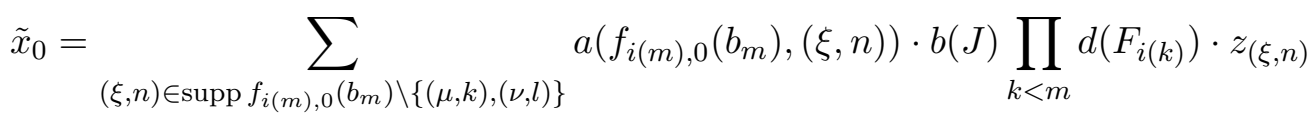

$\mathrm{e}$

$$
x=a\left(f_{i(m), 0}\left(b_{m}\right),(\mu, k)\right) \cdot b(J) \prod_{k<m} d\left(F_{i(k)}\right) \cdot \tilde{z}_{(\mu, k)} .
$$

Se $(\nu, l) \in E_{m} \times e_{m}$, pedimos também que $d\left(F_{i(m)}\right) \cdot z_{(\nu, l)} \in \tilde{\psi}_{m}^{*}(\nu, l)$, onde $\tilde{\psi}_{m}^{*}(\nu, l)$ é o arco aberto de $\mathbb{T}$ centrado no ponto médio de $\psi_{m}^{*}(\nu, l)$ com diâmetro

$$
\delta\left(\tilde{\psi}_{m}^{*}(\nu, l)\right)=\frac{\delta\left(\psi_{m}^{*}(\nu, l)\right)}{4}
$$

Definimos

$$
x_{0}=\tilde{x}_{0}+a\left(f_{i(m), 0}\left(b_{m}\right),(\nu, l)\right) \cdot b(J) \prod_{k<m} d\left(F_{i(k)}\right) \cdot z_{(\nu, l)} .
$$

Temos que $x$ é o ponto médio de $\psi_{m}(i(m), 0)-x_{0}$. Seja $I$ o arco aberto de $\mathbb{T}$ centrado em 
$\tilde{z}_{(\mu, k)} \operatorname{com}$ diâmetro

$$
\delta(I)=\frac{r_{m}}{4 \cdot\left|a\left(f_{i(m), 0}\left(b_{m}\right),(\mu, k)\right)\right| \cdot b(J) \cdot \prod_{k<m+1} d\left(F_{i(k)}\right)} .
$$

Existe $z_{(\mu, k)} \in I$ tal que

$$
a\left(f_{i(m), 1}\left(b_{m}\right),(\mu, k)\right) \cdot b(J) \cdot \prod_{k<m+1} d\left(F_{i(k)}\right) \cdot z_{(\mu, k)}+x_{1} \in \psi_{m}(i(m), 1)
$$

$\mathrm{e}$

$$
a\left(f_{i(m), 0}\left(b_{m}\right),(\mu, k)\right) \cdot b(J) \cdot \prod_{k<m+1} d\left(F_{i(k)}\right) \cdot z_{(\mu, k)}+x_{0} \in \psi_{m}(i(m), 0) .
$$

Para cada $(\xi, n) \in \operatorname{supp} f_{i(m), 1}\left(b_{m}\right)$, seja $\psi_{m+1}^{*}(\xi, n)$ o arco aberto de $\mathbb{T}$ centrado em $z_{(\xi, n)}$ com diâmetro

$$
\delta\left(\psi_{m+1}^{*}(\xi, n)\right)=\frac{r_{m+1}}{b(J) \cdot \prod_{k<m+1} d\left(F_{i(k)}\right)}
$$

e façamos

$$
\psi_{m+1}(\xi, n)=b(J) \cdot \prod_{k<m+1} d\left(F_{i(k)}\right) \cdot \psi_{m+1}^{*}(\xi, n) .
$$

As condições (1), (2), (3) e (4) estão satisfeitas.

Caso 3: $F_{i(m)}$ é do tipo C.

Se $(\xi, n) \in \cup_{2 \leq j<n\left(F_{i(m)}\right)} \operatorname{supp} f_{i(m), j}\left(b_{m}\right)$, procedemos como no caso 1 para definir $\psi_{m+1}^{*}(\xi, n)$ e $\psi_{m+1}(\xi, n)$. Fixemos $(\mu, k) \in \operatorname{supp} f_{i(m), 0}\left(b_{m}\right)$ tal que

$$
\frac{\left|a\left(f_{i(m), 1}\left(b_{m}\right),(\mu, k)\right)\right|}{4} \cdot r_{m}>l_{\zeta_{i(m)}}\left(\frac{r_{m}}{8}\right)
$$

$\mathrm{e}$

$$
\left|\frac{a\left(f_{i(m), 0}\left(b_{m}\right),(\mu, k)\right)}{a\left(f_{i(m), 1}\left(b_{m}\right),(\mu, k)\right)} \cdot l_{\zeta_{i(m)}}\left(\frac{r_{m}}{8}\right)-\zeta_{i(m)} \cdot l_{\zeta_{i(m)}}\left(\frac{r_{m}}{8}\right)\right|<\frac{r_{m}}{8} .
$$

Para cada $(\xi, n) \in \operatorname{supp} f_{i(m), 0}\left(b_{m}\right) \backslash\{(\mu, k)\}$, definimos $z_{(\xi, n)}$ como no caso 1. Sejam, também, $x_{0}$ e $x_{1}$ como no caso 1 . Se $(\mu, k) \in E_{m} \times e_{m}$, seja $\tilde{z}_{(\mu, k)}$ uma raiz $d\left(F_{i(m)}\right)$-ésima do ponto médio de $\psi_{m}^{*}(\mu, k)$. Se $(\mu, k) \notin E_{m} \times e_{m}$, seja $\tilde{z}_{(\mu, k)}$ um elemento arbitrário de $\mathbb{T}$. Consideremos $A$ o arco aberto de $\mathbb{T}$ centrado em $\tilde{z}_{(\mu, k)}$ com diâmetro

$$
\delta(A)=\frac{r_{m}}{4 \cdot b(J) \cdot \prod_{k<m+1} d\left(F_{i(k)}\right)} .
$$


A fim de provar que existe $z_{(\mu, k)} \in A$ tal que

$$
a\left(f_{i(m), 0}\left(b_{m}\right),(\mu, k)\right) \cdot b(J) \cdot \prod_{k<m} d\left(F_{i(k)}\right) \cdot z_{(\mu, k)} \in \psi_{m}(i(m), 0)-x_{0}
$$

e

$$
a\left(f_{i(m), 1}\left(b_{m}\right),(\mu, k)\right) \cdot b(J) \cdot \prod_{k<m} d\left(F_{i(k)}\right) \cdot z_{(\mu, k)} \in \psi_{m}(i(m), 1)-x_{1}
$$

é suficiente mostrar que o conjunto

$$
X=\left\{\left(x+\mathbb{Z}, \frac{a\left(f_{i(m), 0}\left(b_{m}\right),(\mu, k)\right)}{a\left(f_{i(m), 1}\left(b_{m}\right),(\mu, k)\right)} \cdot x+\mathbb{Z}\right): x \in a\left(f_{i(m), 1}\left(b_{m}\right),(\mu, k)\right) \cdot b(J) \cdot \prod_{k<m} d\left(F_{i(k)}\right) \cdot \tilde{A}\right\}
$$

é $r_{m} / 4$-denso em $\mathbb{T}^{2}$, onde $\tilde{A}$ é um intervalo aberto de $\mathbb{R}$ tal que $\tilde{A}+\mathbb{Z}=A$. Temos que

$$
\left\{\left(x+\mathbb{Z}, \frac{a\left(f_{i(m), 0}\left(b_{m}\right),(\mu, k)\right)}{a\left(f_{i(m), 1}\left(b_{m}\right),(\mu, k)\right)} \cdot x+\mathbb{Z}\right): x \in\right] 0, l_{\zeta_{i(m)}}\left(r_{m} / 8\right)[\}
$$

é $r_{m} / 4$-denso em $\mathbb{T}^{2}$, uma vez que

$$
\left|\frac{a\left(f_{i(m), 0}\left(b_{m}\right),(\mu, k)\right)}{a\left(f_{i(m), 1}\left(b_{m}\right),(\mu, k)\right)} \cdot l_{\zeta_{i(m)}}\left(\frac{r_{m}}{8}\right)-\zeta_{i(m)} \cdot l_{\zeta_{i(m)}}\left(\frac{r_{m}}{8}\right)\right|<\frac{r_{m}}{8} .
$$

Logo,

$$
Y=\left\{\left(x+\mathbb{Z}, \frac{a\left(f_{i(m), 0}\left(b_{m}\right),(\mu, k)\right)}{a\left(f_{i(m), 1}\left(b_{m}\right),(\mu, k)\right)} \cdot x+\mathbb{Z}\right): x \in\right] 0,\left|a\left(f_{i(m), 1}\left(b_{m}\right),(\mu, k)\right)\right| \cdot r_{m} / 4[\}
$$

também é $r_{m} / 4$-denso em $\mathbb{T}^{2}$, já que

$$
\frac{\left|a\left(f_{i(m), 1}\left(b_{m}\right),(\mu, k)\right)\right|}{4} \cdot r_{m}>l_{\zeta_{i(m)}}\left(\frac{r_{m}}{8}\right)
$$

Observamos que $d\left(F_{i(m)}\right)=1$, pois $F_{i(m)}$ é do tipo C. Logo,

$$
] 0, \frac{\left|a\left(f_{i(m), 1}\left(b_{m}\right),(\mu, k)\right)\right| \cdot r_{m}}{4}\left[=a\left(f_{i(m), 1}\left(b_{m}\right),(\mu, k)\right) \cdot b(J) \cdot \prod_{k<m} d\left(F_{i(k)}\right) \cdot \tilde{A}+r\right.
$$


para algum $r \in \mathbb{R}$ e, portanto,

$$
Y=X+\left(r+\mathbb{Z}, \frac{a\left(f_{i(m), 0}\left(b_{m}\right),(\mu, k)\right)}{a\left(f_{i(m), 1}\left(b_{m}\right),(\mu, k)\right)} \cdot r+\mathbb{Z}\right)
$$

Como as translações são isometrias de $\mathbb{T}^{2}$, concluímos que $X$ é $r_{m} / 4$-denso em $\mathbb{T}^{2}$. Tomemos $z_{(\mu, k)} \in A$ tal que

$$
a\left(f_{i(m), 0}\left(b_{m}\right),(\mu, k)\right) \cdot b(J) \cdot \prod_{k<m} d\left(F_{i(k)}\right) \cdot z_{(\mu, k)} \in \psi_{m}(i(m), 0)-x_{0}
$$

$\mathrm{e}$

$$
a\left(f_{i(m), 1}\left(b_{m}\right),(\mu, k)\right) \cdot b(J) \cdot \prod_{k<m} d\left(F_{i(k)}\right) \cdot z_{(\mu, k)} \in \psi_{m}(i(m), 1)-x_{1} .
$$

Para cada $(\xi, n) \in \operatorname{supp} f_{i(m), 1}\left(b_{m}\right)$, seja $\psi_{m+1}^{*}(\xi, n)$ o arco aberto de $\mathbb{T}$ centrado em $z_{(\xi, n)}$ com diâmetro

$$
\delta\left(\psi_{m+1}^{*}(\xi, n)\right)=\frac{r_{m+1}}{b(J) \cdot \prod_{k<m+1} d\left(F_{i(k)}\right)}
$$

e façamos

$$
\psi_{m+1}(\xi, n)=b(J) \cdot \prod_{k<m+1} d\left(F_{i(k)}\right) \cdot \psi_{m+1}^{*}(\xi, n) .
$$

As condições (1), (2), (3) e (4) estão satisfeitas.

Por indução, obtemos aplicações $\psi_{m}: E \times \omega \rightarrow \mathcal{B}$ e $\psi_{m}^{*}: E_{m} \times e_{m} \rightarrow \mathcal{B}$ para cada $m \in \omega$, satisfazendo as seguintes condições:

- $\overline{\psi_{m+1}(\xi, n)} \subset \psi_{m}(\xi, n)$, para todo $(\xi, n) \in E \times \omega$;

- $\delta\left(\psi_{m}(\xi, n)\right)=r_{m}$, se $(\xi, n) \in E_{m} \times e_{m}$ e $\psi_{m}(\xi, n)=\mathbb{T}$, se $(\xi, n) \in(E \times \omega) \backslash\left(E_{m} \times e_{m}\right)$;

- $\psi_{m}(\xi, n)=b(J) \cdot \prod_{k<m} d\left(F_{i(k)}\right) \cdot \psi_{m}^{*}(\xi, n)$, se $(\xi, n) \in E_{m} \times e_{m}$

Como $\mathbb{T}$ é um espaço métrico completo e $\left(r_{m}\right)_{m \in \omega}$ é uma seqüência de números reais positivos que converge para 0 , concluímos que se $(\xi, n) \in E \times \omega$, então $\cap_{m \in \omega} \psi_{m}(\xi, n)=\cap_{m \in \omega} \overline{\psi_{m}(\xi, n)}$ é um conjunto unitário. Denotaremos por $\phi\left(\chi_{(\xi, n)}\right)$ o único elemento de $\cap_{m \in \omega} \psi_{m}(\xi, n)$.

Para cada $(\xi, n) \in E \times \omega, \operatorname{sejam} N_{(\xi, n)}=\min \left\{m \in \omega:(\xi, n) \in E_{m} \times e_{m}\right\}$ e $m \geq N_{\xi}$. Temos que $\psi_{m}(\xi, n) \neq \mathbb{T}$ e, portanto, existe um único elemento de $\psi_{m}^{*}(\xi, n)$ que multiplicado por 
$b(J) \cdot \prod_{k<m} d\left(F_{i(k)}\right)$ é igual a $\phi\left(\chi_{(\xi, n)}\right)$. Denotaremos tal elemento por

$$
\phi\left(\frac{1}{b(J) \cdot \prod_{k<m} d\left(F_{i(k)}\right)} \cdot \chi_{(\xi, n)}\right) .
$$

Consideremos

$$
G_{(\xi, n)}=\left\{\frac{1}{b(J) \cdot \prod_{k<m} d\left(F_{i(k)}\right)} \cdot \chi_{(\xi, n)} \in \mathbb{Q}^{(E \times \omega)}: m \geq N_{(\xi, n)}\right\}
$$

e denotemos por $G$ o grupo gerado por $\cup_{(\xi, n) \in E \times \omega} G_{(\xi, n)}$. Uma vez que se $(\xi, n) \in E \times \omega$ e $m>n$, então

$$
\phi\left(\frac{1}{b(J) \cdot \prod_{k<N_{(\xi, n)}} d\left(F_{i(k)}\right)} \cdot \chi_{(\xi, n)}\right)=\prod_{N_{(\xi, n)} \leq k<m} d\left(F_{i(k)}\right) \cdot \phi\left(\frac{1}{b(J) \cdot \prod_{k<m} d\left(F_{i(k)}\right)} \cdot \chi_{(\xi, n)}\right)
$$

podemos estender $\phi$ a um homomorfismo de grupos de $G$ em $\mathbb{T}$. Usando o fato de $\mathbb{T}$ ser divisível, estendemos $\phi$ a um homomorfismo de grupos $\phi_{J} \uparrow_{\mathbb{Q}^{(E \times \omega)}}: \mathbb{Q}^{(E)} \rightarrow \mathbb{T}$.

Temos que

$$
\phi_{J} \uparrow_{\mathbb{Q}^{(E \times \omega)}}(J) \in \sum_{(\xi, n) \in \operatorname{supp} J} a(J,(\xi, n)) \cdot \psi_{0}^{*}(\xi, n) .
$$

Como

$$
0+\mathbb{Z} \notin \sum_{(\xi, n) \in \operatorname{supp} J} a(J,(\xi, n)) \cdot \psi_{0}^{*}(\xi, n)
$$

concluímos que $\phi_{J} \uparrow_{\mathbb{Q}^{(E \times \omega)}}(J) \neq 0+\mathbb{Z}$. Logo, (i) está verificada.

Fixemos $\xi \in E \backslash\{0\}$ e $j<n\left(F_{\xi}\right)$. Para cada $m \in i^{-1}(\{\xi\})$ temos que

$\phi_{J} \uparrow_{\mathbb{Q}^{(E \times \omega)}}\left(f_{i(m), j}\left(b_{m}\right)\right) \in \sum_{(\xi, n) \in \operatorname{supp} f_{i(m), j}\left(b_{m}\right)} a\left(f_{i(m), j}\left(b_{m}\right),(\xi, n)\right) \cdot b(J) \cdot \prod_{k<m} d\left(F_{i(k)}\right) \cdot \psi_{m+1}^{*}(\xi, n)$.

Além disso,

$$
\phi_{J} \Upsilon_{\mathbb{Q}^{(E \times \omega)}}\left(\chi_{(i(m), j)}\right) \in \psi_{m}(i(m), j) .
$$

De (4) segue que

$$
\begin{aligned}
\delta\left(\phi _ { J } \left\lceil_{\mathbb{Q}^{(E \times \omega)}}\left(f_{i(m), j}\left(b_{m}\right)\right), \phi_{J}\left\lceil_{\mathbb{Q}^{(E \times \omega)}}\left(\chi_{(i(m), j)}\right)\right)\right.\right. & \leq d_{1}+d_{2} \\
& <2 r_{m}
\end{aligned}
$$


onde

$$
d_{1}=\delta\left(\sum_{(\xi, n) \in \operatorname{supp} f_{i(m), j}\left(b_{m}\right)} a\left(f_{i(m), j}\left(b_{m}\right),(\xi, n)\right) \cdot b(J) \cdot \prod_{k<m} d\left(F_{i(k)}\right) \cdot \psi_{m+1}^{*}(\xi, n)\right)
$$

$\mathrm{e}$

$$
d_{2}=\delta\left(\psi_{m}(i(m), j)\right)
$$

Como $r_{m} \rightarrow 0$, concluímos que a seqüência $\left\{\phi_{J} \Upsilon_{\mathbb{Q}^{(E \times \omega)}}\left(f_{i(m), j}\left(b_{m}\right)\right): m \in i^{-1}(\{\xi\})\right\}$ converge para $\phi_{J} \uparrow_{\mathbb{Q}^{(E \times \omega)}}\left(\chi_{(i(m), j)}\right)$. Da propriedade (vi) do lema 7.2.2 segue que

$$
\phi_{J} \uparrow_{\mathbb{Q}^{(E \times \omega)}}\left(\chi_{(i(m), j)}\right)=p_{\xi}-\lim \left\{\phi_{J} \Upsilon_{\mathbb{Q}^{(E \times \omega)}}\left(f_{i(m), j}(n)\right): n \in \omega\right\} .
$$

Logo, (ii) está verificada.

Estamos prontos para estender $\phi_{J} \Upsilon_{\mathbb{Q}^{(E \times \omega)}}$ a um homomorfismo de grupos $\phi_{J}: \mathbb{Q}^{(\mathfrak{c} \times \omega)} \rightarrow \mathbb{T}$. Nossa única preocupação será garantir que se $\xi \in \mathfrak{c} \backslash E$, então $\phi_{J}\left(\chi_{(\xi, j)}\right)=p_{\xi}-\lim \left\{\phi_{J}\left(f_{\xi, j}(n)\right)\right.$ : $n \in \omega\}$, para cada $j<n\left(F_{\xi}\right)$.

Lema 7.2.4. Para cada $J \in \mathbb{Q}^{(\mathfrak{c} \times \omega)} \backslash\{0\}$, existe $\phi_{J}: \mathbb{Q}^{(\mathfrak{c} \times \omega)} \rightarrow \mathbb{T}$ um homomorfismo de grupos tal que:

(i) $\phi_{J}(J) \neq 0+\mathbb{Z}$;

(ii) Se $\xi \in] 0, \mathfrak{c}\left[\right.$, então $\phi_{J}\left(\chi_{(\xi, j)}\right)=p_{\xi}-\lim \left\{\phi_{J}\left(f_{\xi, j}(n)\right): n \in \omega\right\}$, para cada $j<n\left(F_{\xi}\right)$.

Demonstração. Faremos a construção de $\phi_{J}$ por indução. De acordo com a proposição 7.2.1, existe $E \in[\mathfrak{c}]^{\omega}$ de modo que supp $J \subset E \times \omega$ e tal que se $\xi \in E \backslash\{0\}$, então $\cup_{n \in \omega} \operatorname{supp} f_{\xi, i}(n) \subset$ $E \times \omega$, para todo $j<n\left(F_{\xi}\right)$. Segundo o lema 7.2.3, é possível construir $\phi_{J} \uparrow_{\mathbb{Q}^{(E \times \omega)}}: \mathbb{Q}^{(E \times \omega)} \rightarrow \mathbb{T}$ um homomorfismo de grupos com as seguintes propriedades:

(1) $\phi_{J} \uparrow_{\mathbb{Q}^{(E \times \omega)}}(J) \neq 0+\mathbb{Z}$

(2) Se $\xi \in E \backslash\{0\}$, então $\phi_{J} \Upsilon_{\mathbb{Q}^{(E \times \omega)}}\left(\chi_{(\xi, j)}\right)=p_{\xi}-\lim \left\{\phi_{J} \Upsilon_{\mathbb{Q}^{(E \times \omega)}}\left(f_{\xi, j}(n)\right): n \in \omega\right\}$, para cada $j<n\left(F_{\xi}\right)$.

Consideremos $\left\{\alpha_{\xi}: \xi<\mathfrak{c}\right\}$ uma indexação estritamente crescente de $\mathfrak{c} \backslash E$. Para cada $j<n\left(F_{\alpha_{0}}\right)$, definamos

$$
\tilde{\phi}_{J}\left(\chi_{\left(\alpha_{0}, j\right)}\right)=p_{\alpha_{0}}-\lim \left\{\phi_{J} \Upsilon_{\mathbb{Q}^{(E \times \omega)}}\left(f_{\alpha_{0}, j}(n)\right): n \in \omega\right\} .
$$


Se $j \geq n\left(F_{\alpha_{0}}\right)$, definamos $\tilde{\phi}_{J}\left(\chi_{\left(\alpha_{0}, j\right)}\right)$ arbitrariamente. Por fim, façamos

$$
\tilde{\phi}_{J}(\tilde{J})=\phi_{J} \uparrow_{\mathbb{Q}^{(E \times \omega)}}(\tilde{J})
$$

para todo $\tilde{J} \in \mathbb{Q}^{(E \times \omega)} .^{5}$

Seja $H_{\alpha_{0}}$ o subgrupo de $\mathbb{Q}^{(\mathfrak{c} \times \omega)}$ gerado por $\mathbb{Q}^{(E \times \omega)} \cup\left\{\chi_{\left(\alpha_{0}, n\right)}: n \in \omega\right\}$. Podemos estender $\tilde{\phi}_{J}$ a um homomorfismo de grupos de $H_{\alpha_{0}}$ em $\mathbb{T}$. Uma vez que $\mathbb{T}$ é divisível, estendemos $\tilde{\phi}_{J}$ a um

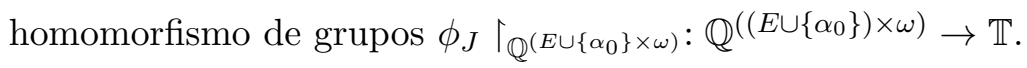

Repetindo indutivamente esta construção, obteremos $\phi_{J}: \mathbb{Q}^{(\mathfrak{c} \times \omega)} \rightarrow \mathbb{T}$ um homomorfismo de grupos satisfazendo (i) e (ii).

\subsection{Explicitando a topologia}

A próxima proposição mostra que o produto diagonal da família $\left\{\phi_{J}: J \in \mathbb{Q}^{(\mathfrak{c} \times \omega)} \backslash\{0\}\right\}$ é um monomorfismo de grupos.

Proposição 7.3.1. Suponhamos que para cada $J \in \mathbb{Q}^{(\mathfrak{c} \times \omega)} \backslash\{0\}$ exista um homomorfismo de grupos $\phi_{J}: \mathbb{Q}^{(\mathfrak{c} \times \omega)} \rightarrow \mathbb{T}$ tal que $\phi_{J}(J) \neq 0+\mathbb{Z}$. A aplicação

$$
\begin{aligned}
\Phi: \mathbb{Q}^{(\mathfrak{c} \times \omega)} & \rightarrow \mathbb{T}^{\mathbb{Q}^{(\mathfrak{c} \times \omega)} \backslash\{0\}} \\
\tilde{J} & \mapsto \quad \Phi(\tilde{J})
\end{aligned}
$$

dada por

$$
\Phi(\tilde{J})(J)=\phi_{J}(\tilde{J}), \text { para cada } J \in \mathbb{Q}^{(\mathfrak{c} \times \omega)} \backslash\{0\}
$$

é um monomorfismo de grupos.

Demonstração. Análoga à da proposição 2.3.1.

Teorema 7.3.2. Existe uma topologia de grupo no grupo abeliano livre de cardinalidade $\mathfrak{c}$ que torna seu quadrado enumeravelmente compacto.

Demonstração. O grupo abeliano livre $\Phi\left[\mathbb{Z}^{(\mathfrak{c} \times \omega)}\right]$ munido da topologia de subespaço induzida por $\mathbb{T}^{\mathbb{Q}^{(c \times \omega)} \backslash\{0\}}$ é um grupo topológico.

\footnotetext{
${ }^{5}$ Estamos considerando $\mathbb{Q}^{(E \times \omega)} \subset \mathbb{Q}^{\left(E \cup\left\{\alpha_{0}\right\} \times \omega\right)} \subset \mathbb{Q}^{(\mathfrak{c} \times \omega)}$.
} 
Sejam $g, h: \omega \rightarrow \Phi\left[\mathbb{Z}^{(\mathfrak{c} \times \omega)}\right]$. De acordo com a proposição 7.1.4, existem $\left.\xi \in\right] 0, \mathfrak{c}[, j: \omega \rightarrow \omega$ estritamente crescente e $\tilde{g}, \tilde{h} \in \mathbb{Z}^{(\mathfrak{c} \times \omega)}$ tais que

$$
\left(\Phi^{-1} \circ g \circ j\right)(n)=\tilde{g}+\sum_{i<n\left(F_{\xi}\right)} a_{\xi, i} \cdot f_{\xi, i}(n)
$$

e

$$
\left(\Phi^{-1} \circ h \circ j\right)(n)=\tilde{h}+\sum_{i<n\left(F_{\xi}\right)} b_{\xi, i} \cdot f_{\xi, i}(n)
$$

para todo $n \in \omega$, onde $a_{\xi, i}, b_{\xi, i} \in \mathbb{Z}$, para todo $i<n\left(F_{\xi}\right)$.

Do lema 7.2.4 segue que

$$
\phi_{J}\left(\chi_{(\xi, i)}\right)=p_{\xi}-\lim \left\{\phi_{J}\left(f_{\xi, i}(n)\right): n \in \omega\right\}
$$

para todo $i<n\left(F_{\xi}\right)$ e todo $J \in \mathbb{Q}^{(\mathfrak{c} \times \omega)} \backslash\{0\}$. Portanto,

$$
\Phi\left(\chi_{(\xi, i)}\right)=p_{\xi}-\lim \left\{\Phi\left(f_{\xi, i}(n)\right): n \in \omega\right\}
$$

para todo $i<n\left(F_{\xi}\right)$. Isto implica que

$$
\Phi\left(\tilde{g}+\sum_{i<n\left(F_{\xi}\right)} a_{\xi, i} \cdot \chi_{(\xi, i)}\right)=p_{\xi}-\lim \left\{\Phi\left(\tilde{g}+\sum_{i<n\left(F_{\xi}\right)} a_{\xi, i} \cdot f_{\xi, i}(n)\right): n \in \omega\right\}
$$

e

$$
\Phi\left(\tilde{h}+\sum_{i<n\left(F_{\xi}\right)} b_{\xi, i} \cdot \chi_{(\xi, i)}\right)=p_{\xi}-\lim \left\{\Phi\left(\tilde{h}+\sum_{i<n\left(F_{\xi}\right)} b_{\xi, i} \cdot f_{\xi, i}(n)\right): n \in \omega\right\}
$$

Logo,

$$
\left(\Phi\left(\tilde{g}+\sum_{i<n\left(F_{\xi}\right)} a_{\xi, i} \cdot \chi_{(\xi, i)}\right), \Phi\left(\tilde{h}+\sum_{i<n\left(F_{\xi}\right)} b_{\xi, i} \cdot \chi_{(\xi, i)}\right)\right)
$$

é um ponto de acumulação de $\{(g(n), h(n)): n \in \omega\}$. Portanto, $\Phi\left[\mathbb{Z}^{(\mathfrak{c} \times \omega)}\right] \times \Phi\left[\mathbb{Z}^{(\mathfrak{c} \times \omega)}\right]$ é enumeravelmente compacto.

\subsection{Sobre o problema de Wallace}

Recordamos que um semigrupo é um conjunto não vazio munido de uma operação binária associativa. Um semigrupo de Wallace é um semigrupo topológico cancelativo (à direita e à 
esquerda) enumeravelmente compacto que não é um grupo topológico. ${ }^{6}$

Teorema 7.4.1. Existe um semigrupo de Wallace cujo quadrado é enumeravelmente compacto.

Demonstração. Temos que $S=\left\{\Phi(J) \in \mathbb{T}^{\mathbb{Q}^{(\mathfrak{c} \times \omega)} \backslash\{0\}}: J \in \mathbb{N}^{(\mathfrak{c} \times \omega)}\right\}$ é um semigrupo topológico cancelativo (à direita e à esquerda) que não é um grupo. Seja $\{(g(n), h(n)): n \in \omega\}$ um subconjunto infinito de $S \times S$, onde $g, h: \omega \rightarrow \Phi\left[\mathbb{N}^{(\mathfrak{c} \times \omega)}\right]$. De acordo com a demonstração da proposição 7.1.4, existem $\xi \in] 0, \mathfrak{c}\left[, j: \omega \rightarrow \omega\right.$ estritamente crecente e $\tilde{g}, \tilde{h} \in \mathbb{N}^{(\mathfrak{c} \times \omega)}$ tais que

$$
\left(\Phi^{-1} \circ g \circ j\right)(n)=\tilde{g}+\sum_{i<n\left(F_{\xi}\right)} a_{\xi, i} \cdot f_{\xi, i}(n)
$$

e

$$
\left(\Phi^{-1} \circ h \circ j\right)(n)=\tilde{h}+\sum_{i<n\left(F_{\xi}\right)} b_{\xi, i} \cdot f_{\xi, i}(n)
$$

para todo $n \in \omega$, onde $a_{\xi, i}, b_{\xi, i} \in \mathbb{N}$ para todo $i<n\left(F_{\xi}\right)$.

Mostramos no teorema 7.3 .2 que $\left(\Phi\left(\tilde{g}+\sum_{i<n\left(F_{\xi}\right)} a_{\xi, i} \cdot \chi_{(\xi, i)}\right), \Phi\left(\tilde{h}+\sum_{i<n\left(F_{\xi}\right)} b_{\xi, i} \cdot \chi_{(\xi, i)}\right)\right)$ é um ponto de acumulação de $\{(g(n), h(n)): n \in \omega\}$. Como $\left(\Phi\left(\tilde{g}+\sum_{i<n\left(F_{\xi}\right)} a_{\xi, i} \cdot \chi_{(\xi, i)}\right), \Phi(\tilde{h}+\right.$ $\left.\left.\sum_{i<n\left(F_{\xi}\right)} b_{\xi, i} \cdot \chi_{(\xi, i)}\right)\right) \in \Phi\left[\mathbb{N}^{(\mathfrak{e} \times \omega)}\right]$, concluímos que $S \times S$ é enumeravelmente compacto.

\footnotetext{
${ }^{6} \mathrm{Um}$ semigrupo $(S, \cdot)$ é dito cancelativo (à direita e à esquerda) se $z \cdot x=z \cdot y$ implica $x=y$ e $x \cdot z=y \cdot z$ implica $x=y$, sempre que $x, y, z \in S$.
} 

CAPÍTULO 8

Considerações finais

\subsection{Resultados obtidos}

Nesta tese, utilizamos técnicas de teoria dos conjuntos para construir topologias enumeravelmente compactas em grupos abelianos de não torção. Assumindo a existência de $\mathfrak{c}$ ultrafiltros seletivos dois a dois incomparáveis segundo a ordem de Rudin-Keisler, obtivemos os seguintes resultados:

(1) O grupo abeliano livre de cardinalidade $\mathfrak{c}$ admite uma topologia enumeravelmente compacta com uma seqüência não trivial convergente que o torna um grupo topológico.

Conforme mencionamos, García-Ferreira e Tomita obtiveram o mesmo resultado assumindo

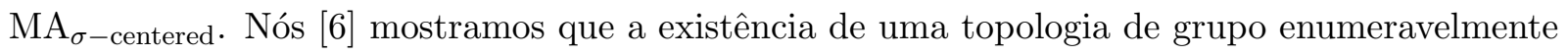
compacta com uma seqüência não trivial convergente no grupo abeliano livre de cardinalidade $\mathfrak{c}$ é compatível com a falha total de MA.

(2) Se $(G,+, \tau)$ é um grupo topológico abeliano quase livre de torção tal que $|G|=|\tau|=\mathfrak{c}$, então existe $\sigma$ uma topologia em $G$ independente de $\tau$ (isto é, tal que $\tau \cap \sigma$ é a topologia cofinita em $G$ ) que torna $(G,+)$ um grupo topológico. 
Tkachenko e Yaschenko [37] obtiveram o mesmo resultado assumindo MA. Todavia, eles afirmaram não saber se era possível obtê-lo assumindo apenas $\mathrm{MA}_{\text {countable. Nós [8] mostramos }}$

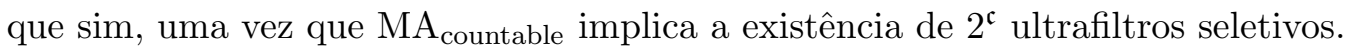

(3) Se $G$ é um grupo abeliano de não torção tal que $|G|=\mathfrak{c}$, então $G$ admite uma topologia de grupo enumeravelmente compacta se, e somente se, $|G / T(G)|=\mathfrak{c}$ e, para quaisquer $n, d \in \mathbb{N}$ tais que $d \mid n$, o conjunto $d G[n]$ é finito ou tem cardinalidade $\mathfrak{c}$.

A caracterização algébrica dos grupos abelianos de não torção e cardinalidade c que admitem uma topologia de grupo enumeravelmente compacta apresentada em (3) havia sido obtida por Dikranjan e Tkachenko [17] sob MA. Nós [7] mostramos que não é necessária qualquer forma do axioma de Martin para obtê-la.

(4) Existe uma topologia de grupo no grupo abeliano livre de cardinalidade $\mathfrak{c}$ que torna seu quadrado enumeravelmente compacto.

Tomita [38] provou que o grupo abeliano livre de cardinalidade $\mathfrak{c}$ não admite uma topologia de grupo que torna sua $\omega$-ésima potência enumeravelmente compacta, mas nada se sabia a respeito de potências finitas. Com (4), nós [9] respondemos uma questão proposta por Madariaga-Garcia e Tomita em [30] e por Tkachenko em [36].

(5) Existe um semigrupo topológico cancelativo que não é um grupo topológico e cujo quadrado é enumeravelmente compacto.

Em outras palavras, nós [9] asseguramos a existência de um semigrupo de Wallace cujo quadrado é enumeravelmente compacto, o que responde uma questão proposta por Grant em [21].

Assumindo a existência de $2^{\mathfrak{c}}$ ultrafiltros seletivos, obtivemos o seguinte resultado:

(6) Se um grupo abeliano de não torção e cardinalidade $\mathfrak{c}$ admite uma topologia de grupo enumeravelmente compacta, então o mesmo admite $2^{\mathfrak{c}}$ topologias enumeravelmente compactas (duas a duas não homeomorfas) que o tornam um grupo topológico.

Assumindo $\mathrm{MA}_{\sigma-\text { centered}}$, Tomita [39] mostrou que o grupo abeliano livre de cardinalidade $\mathfrak{c}$ admite $\mathfrak{c}^{+}$topologias enumeravelmente compactas (duas a duas não homeomorfas) que o tornam um grupo topológico. Nós [7] melhoramos o resultado de [39] em duas direções: em primeiro 
lugar, estamos assumindo uma hipótese mais fraca que o axioma de Martin para ordens parciais $\sigma$-centradas; além disso, ampliamos a classe dos grupos para os quais temos o resultado.

Os resultados enunciados em (2), (3) e (6) referem-se a classes bastante gerais de grupos. Para obtê-los, tivemos de lidar com o fato de os elementos de $\mathbb{T}$ possuírem mais de uma raiz n-ésima, para cada $n>1$ número natural; isto nos levou a considerar uma aplicação auxiliar adicional na construção dos homomorfismos por aproximação. Observamos, também, que a imersão de um grupo abeliano em um grupo divisível não é necessariamente sobrejetora e, portanto, foi preciso tomar um cuidado maior na seleção dos candidatos a pontos de acumulação. Além disso, houve necessidade de considerar seqüências com termos de ordem finita, as quais receberam um tratamento especial. Os resultados enunciados em (1) e (4), por sua vez, dizem respeito ao grupo abeliano livre de cardinalidade $\mathfrak{c}$. A dificuldade em obtê-los reside no fato de fazermos exigências extras sobre a topologia a ser construída: em (1), impomos que a mesma deve ter uma seqüência não trivial convergente e em (4), pedimos que seu quadrado seja enumeravelmente compacto.

\subsection{Problemas abertos}

Problema 8.2.1. (ZFC) O grupo abeliano livre de cardinalidade $\mathfrak{c}$ admite uma topologia de grupo enumeravelmente compacta (sem seqüências não triviais convergentes)?

O problema 8.2.1 aparece, por exemplo, em [12] e em [13] e é considerado uma das grandes questões da área. Madariaga-Garcia e Tomita [30] mostraram que a existência de $\mathfrak{c}$ ultrafiltros seletivos dois a dois incomparáveis (segundo a ordem de Rudin-Keisler) permite muni-lo de uma tal topologia e este é o melhor resultado obtido até então. Eles perguntaram se podemos concluir o mesmo assumindo a existência de apenas um ultrafiltro seletivo.

Problema 8.2.2. Assumindo a existência de um ultrafiltro seletivo, é possível munir o grupo abeliano livre de cardinalidade $\mathfrak{c}$ de uma topologia de grupo enumeravelmente compacta sem seqüências não triviais convergentes?

O próximo problema, proposto por Dikranjan e Shakhmatov em [16] e [15], também relaciona a compacidade enumerável de um grupo topológico com a existência de seqüências não triviais convergentes. Neste trabalho, demos uma resposta afirmativa ao mesmo no caso de um grupo abeliano livre, assumindo a existência de $\mathfrak{c}$ ultrafiltros seletivos dois a dois incomparáveis. 
Problema 8.2.3. Se um grupo abeliano de cardinalidade $\mathfrak{c}$ admite uma topologia de grupo enumeravelmente compacta, então o mesmo admite uma topologia de grupo enumeravelmente compacta com uma seqüência não trivial convergente?

Um grupo abeliano $G$ é dito algebricamente compacto se existe um grupo abeliano $H$ tal que $G \times H$ admite uma topologia de grupo compacta. Os grupos algebricamente compactos formam uma subclasse "estreita" da classe dos grupos abelianos (por exemplo, $\mathbb{Z}$ não é um grupo algebricamente compacto). Por outro lado, todo grupo abeliano $G$ é algebricamente pseudocompacto, ou seja, é possível encontrar um grupo abeliano $H$ tal que $G \times H$ admite uma topologia de grupo pseudocompacta. Em [16], os autores perguntam se todo grupo abeliano $G$ é algebricamente enumeravelmente compacto, isto é, se é possível encontrar um grupo abeliano $H$ tal que $G \times H$ admite uma topologia de grupo enumeravelmente compacta.

Madariaga-Garcia e Tomita [30] e Tkachenko [36] propuseram uma questão semelhante. Eles perguntaram se o grupo abeliano livre de cardinalidade $\mathfrak{c}$ admite uma topologia de grupo que torna seu quadrado enumeravelmente compacto. Neste trabalho, demos uma resposta afirmativa a tal indagação, assumindo a existência de $\mathfrak{c}$ ultrafiltros seletivos dois a dois incomparáveis e utilizando ferramentas até então inusitadas, tais como o teorema de Kronecker para curvas no toro $\mathbb{T}^{2}$ e a caracterização das soluções gerais de equações diofantinas lineares em duas variáveis.

Há interesse em saber que outros grupos abelianos admitem uma tal topologia (e, em particular, são algebricamente enumeravelmente compactos).

Problema 8.2.4. Caracterizar algebricamente os grupos abelianos de não torção que têm cardinalidade $\mathfrak{c}$ e que admitem uma topologia de grupo que torna seu quadrado enumeravelmente compacto.

Em [15], os autores mostraram (via forcing) que a existência de uma topologia de grupo enumeravelmente compacta no grupo abeliano livre de cardinalidade $2^{\mathfrak{c}}$ é consistente (com ZFC). Todavia, nada se sabe a respeito de grupos maiores.

Problema 8.2.5. É possível munir o grupo abeliano livre de cardinalidade $2^{2^{\mathfrak{c}}}$ de uma topologia que o torna um grupo topológico enumeravelmente compacto?

Encerraremos esta seção discorrendo sobre o problema de Scarborough-Stone, o qual relaciona as noções de compacidade seqüencial em produtos e compacidade enumerável no espaço resultante. Recordamos que um espaço topológico $X$ é seqüencialmente compacto se toda seqüência em $X$ possui uma subseqüência convergente. 
Em 1966, Scarborough e Stone [34] perguntaram se qualquer produto de espaços topológicos seqüencialmente compactos é enumeravelmente compacto. Assumindo o axioma diamante $\diamond$, Vaughan [42] construiu uma família de espaços $T_{6}$ seqüencialmente compactos cujo produto não é enumeravelmente compacto. Isto mostra a consistência (com ZFC) de uma resposta negativa para o problema de Scarborough-Stone.

Uma conjunção de resultados de diversos matemáticos — incluindo Vaughan, Nyikos, Soukup, Velicovic e Weiss - soluciona completamente o problema em questão para espaços topológicos satisfazendo determinados axiomas de separação. Eles mostraram que tanto "sim" quanto "não" são respostas consistentes (com ZFC) para o problema de Scarborough-Stone restrito à classe dos espaços $T_{5}$ e $T_{6}$.

A restrição do problema de Scarborough-Stone à classe dos grupos topológicos — citada em [44] e [43], por exemplo — permanece, contudo, aberta.

Problema 8.2.6. (Problema de Scarborough-Stone para grupos topológicos) O produto de grupos topológicos seqüencialmente compactos é enumeravelmente compacto? 



\section{APÊNDICE A}

\section{Ultrafiltros seletivos}

Denotaremos por $\beta \omega$ a compactificação de Stone-Čech do espaço discreto $\omega$, a qual será identificada com o conjunto de todos os ultrafiltros sobre $\omega$ munido da topologia que tem $\{V(A): A \subset \omega\}$ como base de abertos, onde $V(A)=\{u: u$ é um ultrafiltro sobre $\omega$ e $A \in u\}$. Nesta identificação, o espaço discreto $\omega$ corresponde à família dos ultrafiltros principais sobre $\omega$. Representaremos por $\omega^{*}$ a família dos ultrafiltros livres sobre $\omega$ (isto é, $\omega^{*}=\beta \omega \backslash \omega$ ).

Dada $f: \omega \rightarrow \omega$, a função $\beta f: \beta \omega \rightarrow \beta \omega$ definida por

$$
\beta f(p)=\left\{A \subset \omega: f^{-1}(A) \in p\right\}
$$

é denominada a extensão de Stone- $\check{C} e c h$ de $f$.

Definição A.1. Dados $p, q \in \omega^{*}$, dizemos que $p \leq_{R K} q$ se existe uma função $f: \omega \rightarrow \omega$ tal que $\beta f(q)=p$, onde $\beta f$ denota a extensão de Stone- $\check{C}$ ech de $f$. A pré-ordem $\leq_{R K}$ em $\omega^{*}$ é chamada de ordem de Rudin-Keisler. ${ }^{1}$

Definição A.2. Dizemos que $p, q \in \omega^{*}$ são equivalentes (e escrevemos $p \equiv_{R K} q$ ) se existe uma

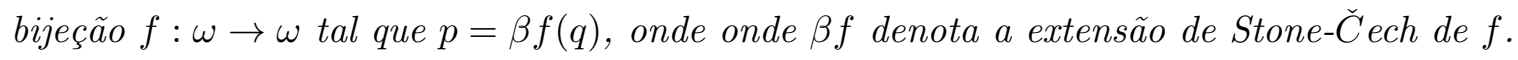

A demonstração do próximo lema foi extraída de [4].

\footnotetext{
${ }^{1}$ Por "pré-ordem", entendemos uma relação reflexiva e transitiva.
} 
Lema A.3. Se $f: \omega \rightarrow \omega$ e $p \in \omega^{*}$ são tais que $\beta f(p)=p$, então $\{n \in \omega: f(n)=n\} \in p$.

Demonstração. Se $x, y \in \omega$, escreveremos $x \sim y$ se, e somente se, existem $m, n \in \omega$ tais que $f^{n}(x)=f^{m}(y){ }^{2}$ Temos que $\sim$ é uma relação de equivalência em $\omega$ e, evidentemente, $f(x) \sim x$. Diremos que $x \in \omega$ é periódico se existir $k \in \mathbb{N} \backslash\{0\}$ tal que $f^{k}(x)=x$. Tomemos $A \subset \omega$ de modo que:

- $|A \cap E|=1$, para todo $E \in \omega / \sim$;

- Se $E$ possui um elemento periódico, então o elemento de $A \cap E$ também é periódico, qualquer que seja $E \in \omega / \sim$.

Fixemos $E \in \omega / \sim$ e denotemos por $a$ o elemento de $A \cap E$. Para cada $x \in E$, sejam

$$
\begin{gathered}
m(x)=\min \left\{m \in \omega: \exists n \in \omega \text { tal que } f^{n}(x)=f^{m}(a)\right\}, \\
n(x)=\min \left\{n \in \omega: f^{n}(x)=f^{m(x)}(a)\right\}
\end{gathered}
$$

$\mathrm{e}$

$$
d(x)=m(x)-n(x) .
$$

Afirmamos que $d(f(x))=d(x)+1$ ou $x=a$. Com efeito, seja $y=f(x)$. Temos que

$$
f^{n(y)+1}(x)=f^{n(y)}(y)=f^{m(y)}(a)
$$

e, portanto, $m(x) \leq m(y)$. Há dois casos a serem considerados:

Caso 1: $m(x)<m(y)$.

Se $n(x) \geq 1$, então $f^{n(x)-1}(y)=f^{n(x)}(x)=f^{m(x)}(a)$ o contradiz a definição de $m(y)$, uma vez que $m(x)<m(y)$. Logo, $n(x)=0$, o que implica $x=f^{m(x)}(a)$ e, portanto, $y=f(x)=f^{m(x)+1}(a)$. Assim, $m(y) \leq m(x)+1$. Como $m(x)<m(y)$, temos que $m(y)=m(x)+1$. Uma vez que $y=f^{m(x)+1}(a)$, concluímos que $n(y)=0$. Dado o exposto, $d(y)=m(y)-n(y)=m(y)=m(x)+1=m(x)-n(x)+1=d(x)+1$.

Caso 2: $m(x)=m(y)$.

Sejam $m=m(x)=m(y)$ e $b=f^{m}(a)$. Como $m(x)=m(y)$ e $f^{n(y)+1}(x)=f^{n(y)}(y)=$ $f^{m(y)}(a)$, temos que $n(x) \leq n(y)+1$. Se $n(x)=n(y)+1$, então $d(y)=d(x)+1$. Suponhamos ${ }^{2} f^{0}=\operatorname{id}_{\omega}$ e $f^{n+1}=f \circ f^{n}$, para todo $n \in \omega$. 
que $n(x)<n(y)+1$. Se $n(x) \geq 1$, então $f^{n(x)-1}(y)=f^{n(x)}(x)=f^{m}(a)$, o que contradiz o fato de $n(x)-1<n(y)$. Logo, $n(x)=0$, o que implica $x=f^{m}(a)=b$. Como $f^{n(y)+1}(b)=f^{n(y)}(y)=b$, temos que $b$ é periódico. Da definição de $A$ segue que $a$ também é periódico e, portanto, $f^{k}(a)=a$, para algum $k \in \mathbb{N} \backslash\{0\}$. Tomemos $p \in \mathbb{N}$ tal que $p k \geq m$ e observamos que $f^{p k-m}(x)=f^{p k-m}\left(f^{m}(a)\right)=f^{p k}(a)=a$. Logo, $m=m(x)=0$ e, portanto, $x=b=a$.

Como $E$ foi tomada arbitrária, podemos considerar $d(x)$, para todo $x \in \omega$. Mostramos acima que $d(f(x))=d(x)+1$, para todo $x \in \omega \backslash A$.

Sejam

$$
X_{0}=\{x \in \omega: d(x) \text { é par }\}
$$

e

$$
X_{1}=\{x \in \omega: d(x) \text { é ímpar }\} \text {. }
$$

Temos que $\omega=X_{0} \cup X_{1}$ e $X_{0} \cap X_{1}=\emptyset$. Como $p \in \omega^{*}$, existe $i \in\{0,1\}$ tal que $X_{i} \in p$. Observamos que $X_{i} \cap f^{-1}\left(X_{i}\right) \subset A$. Uma vez que $\beta f(p)=p$, concluímos que $f^{-1}\left(X_{i}\right) \in p$ e, portanto, $A \in p$. O mesmo argumento mostra que $f^{-1}(A) \in p$, o que implica $A \cap f^{-1}(A) \in p$. Contudo, se $x \in A \cap f^{-1}(A)$, então $x, f(x) \in A$. Da definição de $A$ e do fato de $x \sim f(x)$ decorre que $f(x)=x$. Assim, $A \cap f^{-1}(A) \subset\{x \in \omega: f(x)=x\}$ e daí segue que $\{x \in \omega: f(x)=x\} \in p$.

Proposição A.4. Se $p, q \in \omega^{*}$, então $p \equiv_{R K} q$ se, e somente se, $p \leq_{R K} q$ e $q \leq_{R K} p$.

Demonstração. Se $p \equiv_{R K} q$, então existe $f: \omega \rightarrow \omega$ uma bijeção tal que $\beta f(q)=p$ (e, conseqüentemente, $\left.\beta\left(f^{-1}\right)(p)=q\right)$. Logo, $p \leq_{R K} q$ e $q \leq_{R K} p$. Reciprocamente, suponhamos que $p \leq_{R K} q$ e $q \leq_{R K} p$. Sejam $g, h: \omega \rightarrow \omega$ tais que $\beta g(q)=p$ e $\beta h(p)=q$. Temos que $\beta(g \circ h)(p)=p$ e, de acordo com o lema A.3, o conjunto $A=\{n \in \omega:(g \circ h)(n)=n\}$ é um elemento de $p$. Observamos que $(g \circ h) \uparrow_{A}$ é injetora, o que implica que $h \uparrow_{A}$ também o é. Seja $B \subset A$ tal que $B \in p$ e $|\omega \backslash B|=|\omega \backslash h[B]|=\omega$. Tomemos $f: \omega \rightarrow \omega$ uma bijeção tal que $f \uparrow_{B}=h \uparrow_{B}$. Temos que $\beta f(p)=\beta h(p)=q$. Logo, $p \equiv_{R K} q$.

Dizemos que $p \in \omega^{*}$ é minimal com respeito à ordem de Rudin-Keisler se não existe $q \in \omega^{*}$ tal que $q \leq_{R K} p$ e $p \not \equiv_{R K} q$. Portanto, se $p, q \in \omega^{*}$ são minimais com respeito à ordem de Rudin-Keisler, então $p \equiv_{R K} q$ ou $p$ e $q$ são incomparáveis.

Definição A.5. Um ultrafiltro livre $p$ sobre $\omega$ é dito seletivo se para cada partição $\left\{P_{n}: n \in \omega\right\}$ 
de $\omega$, existe $m \in \omega$ tal que $P_{m} \in p$ ou existe $B \in p$ tal que $\left|B \cap P_{n}\right| \leq 1$, qualquer que seja $n \in \omega .^{3}$

Lema A.6. Seja $p \in \omega^{*}$. As seguintes condições são equivalentes:

(i) p é Rudin-Keisler minimal;

(ii) Para cada $f: \omega \rightarrow \omega$, existe $A \in p$ tal que $f \Gamma_{A}$ é injetora ou constante;

(iii) Se $\left\{P_{n}: n \in \omega\right\}$ é uma partição de $\omega$, então existe $m \in \omega$ tal que $P_{m} \in p$, ou existe $B \in p$ tal que $\left|B \cap P_{n}\right| \leq 1$, para cada $n \in \omega$;

(iv) Para cada coloração $g:[\omega]^{2} \rightarrow\{0,1\}$, existe $C \in p$ tal que $C$ é g-homogêneo (isto é, existe $j \in\{0,1\}$ tal que $g(\{a, b\})=j$, para cada par $\left.\{a, b\} \in[C]^{2}\right)$.

(v) Para cada partição $\left\{P_{0}, P_{1}\right\}$ de $[\omega]^{2}$, existem $D \in p$ e $j \in\{0,1\}$ tais que $[D]^{2} \subset P_{j}$.

Demonstração. Mostraremos que $(i) \Rightarrow(i i) \Rightarrow(i i i) \Rightarrow(i v) \Rightarrow(v) \Rightarrow(i v) \Rightarrow(i i i) \Rightarrow(i)$.

$(i) \Rightarrow(i i)$ : Seja $f: \omega \rightarrow \omega$ uma função. Se $\beta f(p)$ é um ultrafiltro principal sobre $\omega$, então existe $a \in \omega$ tal que $\{a\} \in \beta f(p)$ e, portanto, $f^{-1}(\{a\}) \in p$. Neste caso, temos que $f \uparrow_{A}$ é constante, onde $A=f^{-1}(\{a\})$. Suponhamos que $\beta f(p)$ seja um ultrafiltro livre sobre $\omega$. Temos que $\beta f(p) \leq_{R K} p$ e, por hipótese, $p$ é minimal. Logo, $p \equiv_{R K} \beta f(p)$. Portanto, existe uma permutação $\pi$ de $\omega$ que satisfaz $p=\beta \pi(\beta f(p))=(\beta \pi \circ \beta f)(p)=\beta(\pi \circ f)(p)$. Seja $A=\{n \in \omega:(\pi \circ f)(n)=n\}$. Do lema A.3 segue que $A \in p$. Além disso, $f \uparrow_{A}$ é injetora.

$($ ii $) \Rightarrow($ iii $)$ : Seja $\left\{P_{n}: n \in \omega\right\}$ uma partição de $\omega$ e consideremos $f: \omega \rightarrow \omega$ dada por $f(x)=n$ se, e somente se, $x \in P_{n}$. Por hipótese, existe $A \in p$ tal que $f \uparrow_{A}$ é injetora ou constante. No primeiro caso, temos que $\left|A \cap P_{n}\right| \leq 1$, para todo $n \in \omega$. No segundo, temos que existe $n \in \omega$ tal que $P_{n} \in p$.

$$
\begin{array}{r}
(i i i) \Rightarrow(\text { iv }) \text { : Seja } g:[\omega]^{2} \rightarrow\{0,1\} \text { uma coloração. Definamos } \\
\qquad X_{0}^{0}=\{n \in \omega \backslash\{0\}: g(\{n, 0\})=0\}
\end{array}
$$

$\mathrm{e}$

$$
X_{1}^{0}=\{n \in \omega \backslash\{0\}: g(\{n, 0\})=1\} .
$$

Temos que $\omega \backslash\{0\}=X_{0}^{0} \cup X_{1}^{0}$ e $X_{0}^{0} \cap X_{1}^{0}=\emptyset$. Como $\omega \backslash\{0\} \in p$, existe $i \in\{0,1\}$ tal que $X_{i}^{0} \in p$. Façamos $A_{0}=X_{i}^{0}$ e $l_{0}=i$. Seja $m \in \omega$ e suponhamos definidos $A_{m} \in p$ e $l_{m} \in\{0,1\}$.

\footnotetext{
${ }^{3}$ Dizer que $p \in \omega^{*}$ é seletivo é o mesmo que dizer que, para cada partição $\left\{P_{n}: n \in \omega\right\}$ de $\omega$, existe $m \in \omega$ tal que $P_{m} \in p$ ou existe $B \in p$ tal que $\left|B \cap P_{n}\right|=1$, qualquer que seja $n \in \omega$.
} 


\section{Consideremos}

$$
X_{0}^{m+1}=\left\{n \in A_{m} \backslash(m+1): g(\{n, m+1\})=0\right\}
$$

e

$$
X_{1}^{m+1}=\left\{n \in A_{m} \backslash(m+1): g(\{n, m+1\})=1\right\} .
$$

Novamente, existe $i \in\{0,1\}$ tal que $X_{i}^{m+1} \in p$. Façamos $A_{m+1}=X_{i}^{m+1}$ e $l_{m+1}=i$.

Observamos que $A_{0} \supset A_{1} \supset \ldots \supset A_{n} \supset \ldots$ Sejam $P_{0}=\omega \backslash A_{0}$ e $P_{n+1}=A_{n} \backslash A_{n+1}$, para cada $n \in \omega$. Temos que $\left\{P_{n}: n \in \omega\right\}$ é uma partição de $\omega$ tal que $P_{n} \notin p$, qualquer que seja $n \in \omega$. De (iii) decorre que existe $A \in p$ tal que $\left|A \cap P_{n}\right| \leq 1$, para todo $n \in \omega$. Fixemos um tal $A$ e consideremos $f: \omega \rightarrow \omega$ dada por $f(0)=0$ e

$$
f(n+1)=\max \left\{f(n), \max A \backslash A_{f(n)}\right\}+1
$$

para cada $n \in \omega{ }^{4}$ Uma vez que $f$ é estritamente crescente e $f(0)=0$, temos que $\{[f(n), f(n+1)[: n \in \omega\}$ é uma partição de $\omega$. Como cada elemento desta partição é um subconjunto finito de $\omega$, nenhum deles pertence a $p$. De (iii), novamente, segue que existe $B \in p$ tal que $\mid B \cap[f(n), f(n+1)[\mid \leq 1$, para todo $n \in \omega$. Temos que $A \cap B \in p$.

Sejam $\left\{x_{n}: n \in \omega\right\}$ uma enumeração estritamente crescente de $A \cap B$,

$$
Y_{0}=\left\{x_{2 n}: n \in \omega\right\}
$$

$\mathrm{e}$

$$
Y_{1}=\left\{x_{2 n+1}: n \in \omega\right\}
$$

Tomemos $i \in\{0,1\}$ tal que $Y_{i} \in p$ e façamos $D=Y_{i}$.

Afirmamos que se $m, n \in D$ e $m<n$, então $n \in A_{m}$. Com efeito, da definição de $D$ segue que existe $k \in \omega$ tal que

$$
m<f(k)<f(k+1) \leq n
$$

e, portanto, $n>\max A \backslash A_{f(k)}$. Como $n \in A$, temos que $n \in A_{f(k)}$. Contudo, $A_{m} \supset A_{f(k)}$, uma vez que $m<f(k)$. Logo, $n \in A_{m}$.

Sejam

$$
D_{0}=\left\{n \in D: l_{n}=0\right\} \text {, }
$$

\footnotetext{
${ }^{4}$ Observamos que $A \backslash A_{f(n)}=A \cap\left(P_{0} \cup P_{1} \cup \ldots \cup P_{f(n)}\right)$ e, portanto, este é um conjunto finito.
} 


$$
D_{1}=\left\{n \in D: l_{n}=1\right\}
$$

e $i \in\{0,1\}$ tal que $D_{i} \in p$. Temos que $C=D_{i}$ é $g$-homogêneo. De fato, se $\{m, n\} \in[C]^{2}$ e $m<n$, então $g(\{m, n\})=l_{m}=i$, uma vez que $n \in A_{m}$.

$(i v) \Rightarrow(v)$ : Seja $\left\{P_{0}, P_{1}\right\}$ uma partição de $[\omega]^{2}$. Consideremos a coloração $g:[\omega]^{2} \rightarrow\{0,1\}$ dada por $g(\{x, y\})=0$, se $\{x, y\} \in P_{0}$ e $g(\{x, y\})=1$, se $\{x, y\} \in P_{1}$. Por hipótese, existe $C \in p$ tal que $C$ é $g$-homogêneo e, portanto, existe $j \in 2$ tal que $[C]^{2} \subset P_{j}$.

$(v) \Rightarrow(i v)$ : Seja $g:[\omega]^{2} \rightarrow\{0,1\}$ uma coloração. Consideremos $\left\{P_{0}, P_{1}\right\}$ a seguinte partição de $[\omega]^{2}:\{x, y\} \in[\omega]^{2}$ pertence a $P_{0}$ se $g(\{x, y\})=0$. Caso contrário, $\{x, y\} \in P_{1}$. Por hipótese, existem $D \in p$ e $j \in 2$ tais que $[D]^{2} \subset P_{j}$. Logo, $D$ é $g$-homogêneo.

$(i v) \Rightarrow($ iii $)$ : Seja $\left\{P_{n}: n \in \omega\right\}$ uma partição de $\omega$. Suponhamos que $P_{n} \notin p$, para todo $n \in \omega$. Definamos a coloração $g:[\omega]^{2} \rightarrow\{0,1\}$ da seguinte maneira: $g(\{x, y\})=0$ se $\{x, y\} \subset P_{n}$, para algum $n \in \omega$ e $g(\{x, y\})=1$, caso contrário. Por hipótese, existe $A \in p$ tal que $g$ é constante em $[A]^{2}$. Como estamos supondo que $P_{n} \notin p$, para todo $n \in \omega$, temos que $\left|A \cap P_{n}\right| \leq 1$, para cada $n \in \omega$.

$($ iii $) \Rightarrow(i)$ : Seja $p$ um ultrafiltro livre sobre $\omega$ satisfazendo (3). Vamos mostrar que para cada função $f: \omega \rightarrow \omega$ tal que $\beta f(p) \in \omega^{*}$, temos que $\beta f(p) \equiv_{R K} p$. Seja, portanto, $f$ como acima. Para cada $n \in \omega$, consideremos $P_{n}=f^{-1}(\{n\})$. Temos que $\left\{P_{n}: n \in \omega\right\}$ é uma partição de $\omega$ tal que $P_{n} \notin p$, para todo $n \in \omega$. Por hipótese, existe $A \in p$ tal que $\left|A \cap P_{n}\right| \leq 1$, para cada $n \in \omega$. Daí, segue que $f \uparrow_{A}$ é injetora e, portanto, $\beta f(p) \equiv_{R K} p$.

Lema A.7. [41] Sejam $p$ um ultrafiltro seletivo $e\left\{a_{k}: k \in \omega\right\} \in p$ uma seqüência estritamente crescente de números naturais tal que $k<a_{k}$, para todo $k \in \omega$. Existe $I \subset \omega$ tal que:

(i) $\left\{a_{k}: k \in I\right\} \in p$;

(ii) $\left\{\left[k, a_{k}\right]: k \in I\right\}$ é uma familia de intervalos de $\omega$ dois a dois disjuntos.

Demonstração. Consideremos $A=\left\{a_{k}: k \in \omega\right\} \in p$ uma seqüência estritamente crescente de números naturais tal que $k<a_{k}$, para cada $k \in \omega$. Definamos uma partição $\left\{P_{0}, P_{1}\right\}$ de $[\omega]^{2}$ da seguinte maneira: para cada $\{x, y\} \in[\omega]^{2},\{x, y\}$ é um elemento de $P_{0}$ se, e somente se, existem $i, k \in \omega$, com $i<k$, tais que $\{x, y\}=\left\{a_{i}, a_{k}\right\}$ e $a_{i}<k$. Como $p$ é um ultrafiltro seletivo, do lema A.6 decorre que existe $B \in p$ tal que $B \subset A$ e $[B]^{2} \subset P_{0}$ ou $[B]^{2} \subset P_{1}$.

Afirmamos que $[B]^{2} \not \subset P_{1}$. Com efeito, seja $\left\{t_{n}: n \in \omega\right\}$ uma seqüência estritamente crescente de números naturais tal que $B=\left\{a_{t_{n}}: n \in \omega\right\}$. Se $\left\{a_{t_{0}}, a_{t_{n}}\right\} \in P_{1}$, então $a_{t_{0}} \geq t_{n}$ para todo $n>0$, o que é uma contradição. Portanto, $[B]^{2} \subset P_{0}$. Assim, se $\left\{a_{i}, a_{k}\right\} \in[B]^{2} \operatorname{com} a_{i}<a_{k}$, 
então $i<a_{i}<k<a_{k}$. Seja $I \subset \omega$ tal que $B=\left\{a_{k}: k \in I\right\}$. As condições (i) e (ii) são satisfeitas.

Lema A.8. [41] Sejam $p_{0}$ e $p_{1}$ ultrafiltros seletivos incomparáveis (segundo a ordem de RudinKeisler). Para cada $j \in 2$, seja $\left\{a_{k}^{j}: k \in \omega\right\} \in p_{j}$ uma seqüência estritamente crescente de números naturais tal que $k<a_{k}^{j}$, qualquer que seja $k \in \omega$. Existem $I_{0}$ e $I_{1}$ subconjuntos disjuntos de $\omega$ tais que:

(i) $\left\{a_{k}^{j}: k \in I_{j}\right\} \in p_{j}$, para cada $j \in 2$;

(ii) $\left\{\left[k, a_{k}^{j}\right]: j \in 2, k \in I_{j}\right\}$ é uma família de intervalos de $\omega$ dois a dois disjuntos.

Demonstração. De acordo com o lema A.7, existem $J_{0}, J_{1} \subset \omega$ tais que

(1) $B_{j}=\left\{a_{k}^{j}: k \in J_{j}\right\} \in p_{j}$, para cada $j \in 2$;

(2) $\left\{\left[k, a_{k}^{j}\right]: k \in J_{j}\right\}$ é uma família de intervalos de $\omega$ dois a dois disjuntos, para cada $j<2$.

Uma vez que $p_{0}$ e $p_{1}$ são ultrafiltros distintos, podemos assumir que

(3) $B_{0} \cap B_{1}=\emptyset .^{5}$

Nosso objetivo é encontrar $I_{0} \subset J_{0}$ e $I_{1} \subset J_{1}$ subconjuntos disjuntos de $\omega$ de modo a satisfazer os ítens (i) e (ii). De acordo com (2), o item (ii) estará satisfeito se valer

(4) $\left[i, a_{i}^{0}\right] \cap\left[k, a_{k}^{1}\right]=\emptyset$, quaisquer que sejam $i \in I_{0}$ e $k \in I_{1} \cdot{ }^{6}$

Da seletividade de $p_{1}$ e de (3) decorre que existe $C_{1} \in p_{1}$ tal que $C_{1} \subset B_{1}$ e tal que se $a$ e $b$ são elementos consecutivos de $B_{0}$, então $\left|[a, b] \cap C_{1}\right| \leq 1$. Seja

$$
T=\bigcup\left\{\left[i, a_{i}^{0}\right]: a_{i}^{0} \in B_{0} \text { e }\left[i, a_{i}^{0}\right] \cap C_{1} \neq \emptyset\right\} .
$$

Se $n \in T$, existe um único $t \in \omega$ tal que $n \in\left[t, a_{t}^{0}\right]$ e existe um único $m \in \omega$ tal que $\{m\}=\left[t, a_{t}^{0}\right] \cap C_{1}$. Façamos $f_{0}(n)=m$ para cada $n \in T$ e definamos $f_{0}$ em $\omega \backslash T$ arbitrariamente.

Uma vez que $\beta f_{0}\left(p_{0}\right) \neq p_{1}$ (pois $p_{0}$ e $p_{1}$ são incomparáveis), existe $D_{1} \in p_{1}$ tal que $D_{1} \subset C_{1}$ e $f_{0}^{-1}\left(D_{1}\right) \notin p_{0}$. Seja $C_{0}=B_{0} \backslash f_{0}^{-1}\left(D_{1}\right) \in p_{0}$.

\footnotetext{
${ }^{5}$ De fato, se tomarmos $C \in p_{0} \backslash p_{1}$, podemos trabalhar com os conjuntos $B_{0} \cap C \in p_{0}$ e $B_{1} \cap(\omega \backslash C) \in p_{1}$.

${ }^{6}$ Se valer (4), então $I_{0}$ e $I_{1}$ serão disjuntos.
} 
Afirmamos que se $a_{i}^{0} \in C_{0}$, então $\left[i, a_{i}^{0}\right] \cap D_{1}=\emptyset$. De fato, se $a_{t}^{1} \in\left[i, a_{i}^{0}\right] \cap D_{1}$, então $\left\{a_{t}^{1}\right\}=\left[i, a_{i}^{0}\right] \cap D_{1}=\left[i, a_{i}^{0}\right] \cap C_{1}$ e, portanto, $f_{0}\left(a_{i}^{0}\right)=a_{t}^{1} \in D_{1}$. Assim, $a_{i}^{0} \in f_{0}^{-1}\left(D_{1}\right)$, o que contradiz a definição de $C_{0}$.

Usando a seletividade de $p_{0}$, podemos encontrar $D_{0} \in p_{0}$ tal que $D_{0} \subseteq C_{0}$ e tal que se $a$ e $b$ são elementos consecutivos de $D_{1}$, então $\left|[a, b] \cap D_{0}\right| \leq 1$.

Por um argumento análogo, concluímos que existem $E_{0} \in p_{0}, E_{1} \in p_{1}$ tais que:

(5) $E_{j} \subset D_{j}$, para cada $j \in 2$;

(6) Se $a_{t}^{1} \in E_{1}$, então $\left[t, a_{t}^{1}\right] \cap E_{0}=\emptyset$.

(7) Se $a_{i}^{0} \in E_{0}$, então $\left[i, a_{i}^{0}\right] \cap E_{1}=\emptyset$.

Seja $I_{j} \subset \omega$ tal que $E_{j}=\left\{a_{k}^{j}: k \in I_{j}\right\}$, para cada $j \in 2$. Do item (5), segue que $I_{0}$ e $I_{1}$ satisfazem (i). Conforme observado, para mostrar que vale (ii), é suficiente mostrar que vale (4). Portanto, suponhamos por absurdo que existam $i \in I_{0}$ e $t \in I_{1}$ tais que $\left[i, a_{i}^{0}\right] \cap\left[t, a_{t}^{1}\right] \neq \emptyset$. Então, $a_{i}^{0} \in\left[t, a_{t}^{i}\right]$ ou $a_{t}^{1} \in\left[i, a_{i}^{0}\right]$, o que contradiz (6) e (7). Logo, (ii) está satisfeita.

Lema A.9. [40] Seja $\left\{p_{j}: j \in \omega\right\}$ uma familia de ultrafiltros seletivos dois a dois incomparáveis (segundo a ordem de Rudin-Keisler). Para cada $j \in \omega$, seja $\left\{a_{k}^{j}: k \in \omega\right\} \in p_{j}$ uma seqüência estritamente crescente de números naturais tal que $k<a_{k}^{j}$, qualquer que seja $k \in \omega$. Existe $\left\{I_{j}: j \in \omega\right\}$ uma familia de subconjuntos de $\omega$ dois a dois disjuntos tal que:

(i) $\left\{a_{k}^{j}: k \in I_{j}\right\} \in p_{j}$, para cada $j \in \omega$;

(ii) $\left\{\left[k, a_{k}^{j}\right]: j \in \omega, k \in I_{j}\right\}$ é uma familia de intervalos de $\omega$ dois a dois disjuntos.

Demonstração. Por indução, vamos construir para quaisquer $j, t \in \omega$, um subconjunto $I_{j, t}$ de $\omega$ tal que:

(1) $I_{j, t+1} \subset I_{j, t}$, para cada $t \in \omega$;

(2) $\left\{\left[k, a_{k}^{j}\right]: j \leq t, k \in I_{j, t}\right\}$ é uma família de intervalos de $\omega$ dois a dois disjuntos, para todo $t \in \omega ;$

(3) $\left\{a_{k}^{j}: k \in I_{j, t}\right\} \in p_{j}$, quaisquer que sejam $j, t \in \omega$. 
Do lema A.7 segue que existe $I_{0,0} \subset \omega$ tal que $\left\{a_{k}^{0}: k \in I_{0,0}\right\} \in p_{0}$ e $\left\{\left[k, a_{k}^{j}\right]: k \in I_{0,0}\right\}$ é uma família de intervalos de $\omega$ dois a dois disjuntos.

Suponha que $I_{j, t}$ tenha sido definido para $j, t \leq s$ de modo a satisfazer as condições (1), (2) e (3). Seja $I_{s+1,0}=\omega$. Resta definir $I_{s+1, t+1}$ e $I_{t, s+1}$, para $0 \leq t \leq s$. Aplicamos o lema A.8 para $\left\{a_{k}^{s+1}: k \in I_{s+1,0}\right\}$ e $\left\{a_{k}^{0}: k \in I_{0, s}\right\}$, obtendo $I_{s+1,1}$ e $I_{0, s+1}$. Em seguida, aplicamos novamente o lema A.8 para $\left\{a_{k}^{s+1}: k \in I_{s+1,1}\right\}$ e $\left\{a_{k}^{1}: k \in I_{1, s}\right\}$, obtendo $I_{s+1,2}$ e $I_{1, s+1}$. Continuamos com este processo até aplicarmos o lema A.8 para $\left\{a_{k}^{s+1}: k \in I_{s+1, s}\right\}$ e $\left\{a_{k}^{s}: k \in I_{s, s}\right\}$, obtendo assim $I_{s+1, s+1}$ e $I_{s, s+1}$. Observamos que:

- $I_{t, s+1} \subset I_{t, s}$ e $I_{s+1, t+1} \subset I_{s+1, t}$, para todo $t \leq s$;

- $\left\{\left[k, a_{k}^{t}\right]: k \in I_{t, s+1}\right\} \cup\left\{\left[k, a_{k}^{s+1}\right]: k \in I_{s+1, t+1}\right\}$ é uma família de intervalos de $\omega$ dois a dois disjuntos;

- $\left\{a_{k}^{t}: k \in I_{t, s+1}\right\} \in p_{t}$ e $\left\{a_{k}^{s+1}: k \in I_{s+1, t+1}\right\} \in p_{s+1}$, para todo $t \leq s$.

Temos que (1), (2) e (3) são satisfeitas para quaisquer $j, t \leq s+1$. Portanto, podemos definir $I_{j, t}$ satisfazendo (1), (2) e (3) para quaisquer $j, t \in \omega$.

Para cada $j \in \omega, p_{j}$ é seletivo. De acordo com (3), existe $K_{j} \subset I_{j, j}$ tal que:

(4) $K_{j} \backslash I_{j, t}$ é finito, para cada $t \in \omega$;

(5) $\left\{a_{k}^{j}: k \in K_{j}\right\} \in p_{j}$.

De (4) decorre que existe $M_{j} \in \omega$ tal que:

(6) $K_{l} \subset I_{l, j} \cup M_{j}$, para todo $l \leq j$;

Seja

(7) $I_{j}=K_{j} \backslash \max \left\{a_{k}^{l}+1: l<j, k<M_{j}\right\}$.

De acordo com (5), $I_{j}$ satisfaz (i), para cada $j \in \omega$.

Sejam $j, j^{\prime}, k, k^{\prime} \in \omega$ com $k \in I_{j}$ e $k^{\prime} \in I_{j^{\prime}}$. Se $j=j^{\prime}$, dos ítens (7) e (4) decorre que $k$, $k^{\prime} \in I_{j} \subset I_{j, j}$. De (2) segue que $\left[k, a_{k}^{j}\right] \cap\left[k^{\prime}, a_{k^{\prime}}^{j}\right]=\emptyset$. Podemos assumir, portanto, que $j^{\prime}<j$. Temos que $k \in I_{j, j}$. Se $k^{\prime} \in I_{j^{\prime}, j}$, de $(2)$ segue que $\left[k, a_{k}^{j}\right] \cap\left[k^{\prime}, a_{k^{\prime}}^{j^{\prime}}\right]=\emptyset$. Se $k^{\prime} \notin I_{j^{\prime}, j}$, então $k^{\prime} \in M_{j}$, pois $k^{\prime} \in I_{j^{\prime}} \subset K_{j^{\prime}} \subset I_{j^{\prime}, j} \cup M_{j}$. De (7) segue que $k>a_{k^{\prime}}^{j^{\prime}}$. Logo, $\left[k, a_{k}^{j}\right] \cap\left[k^{\prime}, a_{k^{\prime}}^{j^{\prime}}\right]=\emptyset$. 



\section{APÊNDICE B}

\section{Ultrafiltros seletivos e o axioma de Martin}

Seja $(\mathbb{P}, \leq)$ um conjunto parcialmente ordenado. Dizemos que $p, q \in \mathbb{P}$ são incompatíveis se não existe $r \in \mathbb{P}$ tal que $r \leq p$ e $r \leq q$. Um subconjunto $A$ de $\mathbb{P}$ é uma anticadeia se quaisquer dois elementos distintos de $A$ forem incompatíveis. Dizemos que $D \subset \mathbb{P}$ é denso se, para cada $p \in \mathbb{P}$, existe $q \in D$ tal que $q \leq p$. Por fim, $G \subset \mathbb{P}$ é dito um filtro genérico para uma família $\mathcal{D}$ de subconjuntos densos de $\mathbb{P}$ se:

(1) $\forall p, q \in G \exists r \in G(r \leq p$ e $r \leq q)$;

(2) $\forall p \in G \forall q \in \mathbb{P}(p \leq q \rightarrow q \in G)$;

(3) $D \in \mathcal{D} \rightarrow G \cap D \neq \emptyset$.

O axioma de Martin (MA) afirma que se $\mathbb{P}$ é um conjunto parcialmente ordenado de modo que todas as suas anticadeias sejam enumeráveis, então para cada família $\mathcal{D}$ de subconjuntos densos de $\mathbb{P}$ tal que $|\mathcal{D}|<\mathfrak{c}$, existe um filtro genérico.

O axioma de Martin para ordens parciais enumeráveis ( $\mathrm{MA}_{\text {countable }}$ ) afirma, por sua vez, que se $\mathbb{P}$ é um conjunto parcialmente ordenado enumerável, então para cada família $\mathcal{D}$ de subconjuntos densos de $\mathbb{P}$ tal que $|\mathcal{D}|<\mathfrak{c}$, existe um filtro genérico.

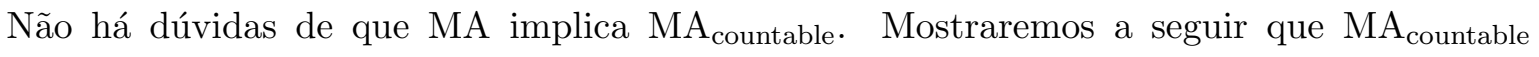
implica a existência de $2^{\mathfrak{c}}$ ultrafiltros seletivos (dois a dois incomparáveis segundo a ordem de 
Rudin-Keisler em $\omega^{*}$ ). A demonstração que apresentaremos aqui deve-se a A. Blass e encontra-se em $[5] .^{1}$

Lema B.1. ( $\left.\mathrm{MA}_{\text {countable }}\right)$ Se $\mathfrak{B}$ é base para um ultrafiltro livre sobre $\omega$, então $|\mathfrak{B}|=\mathfrak{c}$.

Demonstração. Por absurdo, suponhamos que exista $\mathfrak{B}$ uma base para um ultrafiltro livre sobre $\omega$ tal que $|\mathfrak{B}|<\mathfrak{c}$. Afirmamos que existem $S_{0}, S_{1} \subset \omega$ infinitos e disjuntos tais que $S_{0} \cap B \neq \emptyset$ e $S_{1} \cap B \neq \emptyset$, qualquer que seja $B \in \mathfrak{B}$. Com efeito, consideremos $\mathbb{P}=\operatorname{Fn}(\omega, 2)$ ordenado pela inclusão reversa (isto é, se $p, q \in \mathbb{P}$, então $p \leq q$ se, e somente, se $p \supset q$ ). Para cada $n \in \omega$, seja

$$
D_{n}=\{p \in \mathbb{P}: n \in \operatorname{dom} p\}
$$

e, dados $B \in \mathfrak{B}$ e $m \in \omega$, consideremos

$$
D_{B, 0}^{m}=\{p \in \mathbb{P}: \exists n \in B \backslash m \text { tal que } p(n)=0\}
$$

$\mathrm{e}$

$$
D_{B, 1}^{m}=\{p \in \mathbb{P}: \exists n \in B \backslash m \text { tal que } p(n)=1\} .
$$

Como $\mathfrak{B}$ é base para um ultrafiltro livre sobre $\omega$, os elementos de $\mathfrak{B}$ são subconjuntos infinitos de $\omega$ e, portanto,

$$
\mathcal{D}=\left\{D_{n}: n \in \omega\right\} \cup\left\{D_{B, 0}^{m}: B \in \mathfrak{B}, m \in \omega\right\} \cup\left\{D_{B, 1}^{m}: B \in \mathfrak{B}, m \in \omega\right\}
$$

é uma família de subconjuntos densos de $\mathbb{P}$ tal que $|\mathcal{D}| \leq \max \{\omega,|\mathfrak{B}|\}<\mathfrak{c}$. Como $|\mathbb{P}|=\omega$,

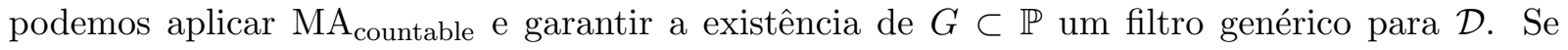
$f=\bigcup G$, então que $f \in \omega^{\omega}$. Definimos $S_{1}=f^{-1}(\{0\})$ e $S_{2}=f^{-1}(\{1\})$. Temos que $S_{0}$ e $S_{1}$ são subconjuntos infinitos de $\omega$ tais que $S_{0} \cap B \neq \emptyset$ e $S_{1} \cap B \neq \emptyset$, qualquer que seja $B \in \mathfrak{B}$.

Como $\mathfrak{B}$ gera um ultrafiltro sobre $\omega$ e tanto $S_{0}$ quanto $S_{1}$ intersecta todo elemento de $\mathfrak{B}$, então $S_{0}$ e $S_{1}$ pertencem ao ultrafiltro em questão, o que é absurdo, uma vez que $S_{0} \cap S_{1}=\emptyset$.

Teorema B.2. (MA countable) Existem $2^{\mathfrak{c}}$ ultrafiltros seletivos.

\footnotetext{
${ }^{1}$ Em [5], o autor mostra que MA implica a existência de $2^{\mathfrak{c}}$ ultrafiltros seletivos (dois a dois incomparáveis segundo a ordem de Rudin-Keisler em $\omega^{*}$ ). Como estamos interessados em obter o mesmo resultado partindo de

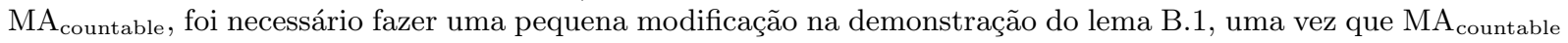
não garante que $\mathfrak{p}=\mathfrak{c}$. Achamos, também, mais adequado reescrever a demonstração do teorema B.2 usando a notação de árvore binária.
} 
Demonstração. Seja $\left\{f_{\alpha}: \alpha<\mathfrak{c}\right\}$ uma indexação do conjunto de todas as funções $f: \omega \rightarrow \omega$ de modo que se $\alpha<\mathfrak{c}$ é um ordinal limite, então $f_{\alpha}$ é uma função limitada. Para cada $\alpha<\mathfrak{c}$ e cada $\varphi \in{ }^{\mathfrak{c}} 2$, vamos definir $\mathcal{Y}_{\alpha}^{\varphi}$ como sendo uma base para algum filtro sobre $\omega$ tal que:

(1) $\mathcal{Y}_{\alpha}^{\varphi}$ contém todos os subconjuntos cofinitos de $\omega$;

(2) Se $\beta<\alpha$, então $\mathcal{Y}_{\beta}^{\varphi} \subset \mathcal{Y}_{\alpha}^{\varphi}$;

(3) $\left|\mathcal{Y}_{\alpha}^{\varphi}\right| \leq \max \{\omega,|\alpha|\}$

(4) $f_{\alpha}$ é limitada ou injetora em algum elemento de $\mathcal{Y}_{\alpha}^{\varphi}$;

(5) Se $\alpha$ é o primeiro ordinal em que $\varphi: \mathfrak{c} \rightarrow 2$ e $\phi: \mathfrak{c} \rightarrow 2$ diferem, $\mathcal{Y}_{\alpha}^{\varphi}=\mathcal{Y}_{\alpha}^{\psi}$, mas $\mathcal{Y}_{\alpha+1}^{\varphi}$ possui um elemento disjunto de algum elemento de $\mathcal{Y}_{\alpha+1}^{\psi}$.

Se $\varphi \in{ }^{0} 2$, definimos $\mathcal{Y}_{0}^{\varphi}$ como sendo a família de todos os subconjuntos cofinitos de $\omega$. Fixemos $\alpha<\mathfrak{c}$ um ordinal e suponhamos construído $\mathcal{Y}_{\beta}^{\varphi}$ para cada $\beta<\alpha$ e cada $\varphi \in{ }^{\beta} 2$. Vamos definir $\mathcal{Y}_{\alpha}^{\varphi}$ para cada $\varphi \in{ }^{\alpha}$ 2. Se $\alpha<\mathfrak{c}$ é um ordinal limite e $\varphi \in{ }^{\alpha} 2$, façamos $\mathcal{Y}_{\alpha}^{\varphi}=\cup_{\beta<\alpha} \mathcal{Y}_{\beta}^{\left.\varphi\right|_{\beta}}$. Suponhamos que $\alpha<\mathfrak{c}$ seja um ordinal sucessor - digamos, $\alpha=\beta+1-$ e fixemos $\varphi \in{ }^{\alpha} 2$. Vamos construir $\mathfrak{B}$ uma base para um filtro sobre $\omega$ com as seguintes propriedades:

- $\mathfrak{B} \supset \mathcal{Y}_{\beta}^{\varphi \Gamma_{\beta}}$;

- $|\mathfrak{B}| \leq \max \{\omega,|\alpha|\}<\mathfrak{c}$

- $f_{\alpha}$ é limitada ou injetora em algum elemento de $\mathfrak{B} .^{2}$

Se $f_{\alpha}$ é limitada em algum elemento de $\mathcal{Y}_{\beta}^{\varphi \Gamma_{\beta}}$, façamos $\mathfrak{B}=\mathcal{Y}_{\beta}^{\varphi \Gamma_{\beta}}$. Suponhamos, portanto, que $f_{\alpha}$ seja ilimitada em todo elemento de $\mathcal{Y}_{\beta}^{\varphi_{\beta}}$. Seja $\mathbb{P}$ a família de todos os subconjuntos finitos de $\omega$ nos quais $f_{\alpha}$ é injetora, ordenada pela inclusão reversa. Temos que $\mathbb{P}$ é enumerável. Para cada $Y \in \mathcal{Y}_{\beta}^{\varphi \Gamma_{\beta}}$, seja $D_{Y}=\{p \in \mathbb{P}: p \cap Y \neq \emptyset\}$. Temos que $D_{Y}$ é denso em $\mathbb{P}$, uma vez que $f_{\alpha}$ é

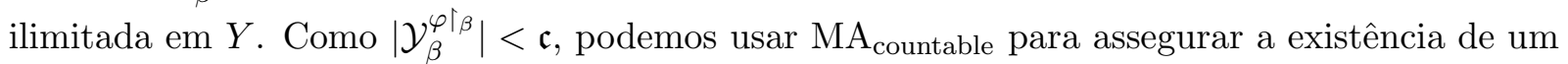
filtro $G$ em $\mathbb{P}$ tal que $G \cap D_{Y} \neq \emptyset$, para todo $Y \in \mathcal{Y}_{\beta}^{\left.\varphi\right|_{\beta}}$. Seja $T=\bigcup G$. Como $G$ é filtro, temos que $f_{\alpha}$ é injetora em $T$. Como $G \cap D_{Y} \neq \emptyset$ para todo $Y \in \mathcal{Y}_{\beta}^{\left.\varphi\right|_{\beta}}$, temos que $T$ intersecta todo elemento de $\mathcal{Y}_{\beta}^{\left.\varphi\right|_{\beta}}$. Seja $\mathfrak{B}$ a base para um filtro sobre $\omega$ obtida adicionando $T$ a $\mathcal{Y}_{\beta}^{\varphi \uparrow_{\beta}}$ e fechando a família resultante por intersecções finitas.

\footnotetext{
${ }^{2} \mathrm{O}$ conjunto $\mathfrak{B}$ funcionará quase como $\mathcal{Y}_{\alpha}^{\varphi}$, já que a condição $(5)$ pode falhar.
} 
Como $\mathfrak{B}$ contém todos os subconjuntos cofinitos de $\omega$ e $|\mathfrak{B}|<\mathfrak{c}$, do lema B.1 decorre que o filtro gerado por $\mathfrak{B}$ não é um ultrafiltro sobre $\omega$. Portanto, existe $S \subset \omega$ tal que $S$ e $\omega \backslash S$ intersectam todo elemento de $\mathfrak{B}$. Fixemos um tal $S$. Se $\varphi(\beta)=0$, adicionamos a $\mathfrak{B}$ o conjunto $S$ e fechamos esta família por intersecções finitas, a fim de obter $\mathcal{Y}_{\alpha}^{\varphi}$. Se $\varphi(\beta)=1$, fazemos o mesmo para o conjunto $\omega \backslash S$.

Para cada $\alpha<\mathfrak{c}$ e cada $\varphi \in{ }^{\mathfrak{c}} 2$, definimos $\mathcal{Y}_{\alpha}^{\varphi}=\mathcal{Y}_{\alpha}^{\varphi}{ }^{\mid \alpha}$. As condições (1), (2), (3), (4) e (5) estão satisfeitas. Se $\varphi \in{ }^{\mathfrak{c}} 2$, tomemos $D^{\varphi}$ um ultrafiltro contendo $\cup_{\alpha<\mathfrak{c}} \mathcal{Y}_{\alpha}^{\varphi}$. De (1) segue que $D^{\varphi}$ é livre. De (4) segue que $D^{\varphi}$ é seletivo. ${ }^{3}$ De (5) vem que $D^{\varphi} \neq D^{\psi}$, se $\varphi \neq \psi$.

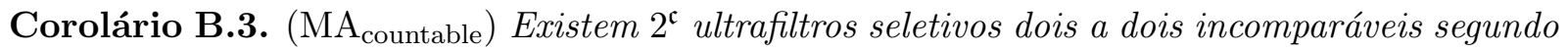
a ordem de Rudin-Keisler em $\omega^{*}$.

Demonstração. Do teorema B.2 segue que existem $2^{\mathfrak{c}}$ ultrafiltros seletivos. Sabemos que quaisquer dois deles são incomparáveis ou equivalentes. Como existem apenas c bijeções de $\omega$ em $\omega$, temos que cada classe de equivalência possui no máximo $\mathfrak{c}$ elementos e, portanto, devemos ter $2^{\mathfrak{c}}$ dessas classes, cada uma tendo um ultrafiltro seletivo como representante. Logo, existem $2^{\mathfrak{c}}$ ultrafiltros seletivos dois a dois incomparáveis segundo a ordem de Rudin-Keisler em $\omega^{*}$.

\footnotetext{
${ }^{3}$ Observamos que se $\mathfrak{U}$ é um ultrafiltro sobre $\omega, A \in \mathfrak{U}$ e $f: \omega \rightarrow \omega$ é limitada em $A$, então existe $B \in \mathfrak{U}$ tal que $f \uparrow_{B}$ é constante. Com efeito, $F=\left\{n \in \omega: f^{-1}(\{n\}) \cap A \neq \emptyset\right\}$ é um conjunto finito e $A \subset \cup_{n \in F} f^{-1}(\{n\})$. Se $f^{-1}(\{n\}) \notin \mathfrak{U}$ para cada $n \in \omega$, então $\omega \backslash A=\cap_{n \in D}\left(\omega \backslash f^{-1}(\{n\})\right) \in \mathfrak{U}$, o que é absurdo. Logo, existe $n \in F$ tal que $B=f^{-1}(\{n\}) \in \mathfrak{U}$. Claramente, $f \uparrow_{B}$ é constante.
} 


\section{Referências Bibliográficas}

[1] A. Arhangel'skii and M. Tkachenko, Topological groups and related structures, Atlantis Press, 2008.

[2] J. E. Baumgartner, Sacks forcing and the total failure of Martin's axiom, Topology Appl. 19 (1985), 211-225.

[3] A. R. Bernstein, A new kind of compactness for topological spaces, Fund. Math. 66 (1970), $185-193$.

[4] A. R. Blass, Orderings of ultrafilters, Ph.D. thesis, Harvard University, 1970.

[5] _ , The Rudin-Keisler ordering of p-points, Trans. Am. Math. Soc. 179 (1973), 145166.

[6] A. C. Boero, S. Garcia-Ferreira, and A. H. Tomita, A countably compact free Abelian group of size continuum that admits a non-trivial convergent sequence, submitted.

[7] A. C. Boero and A. H. Tomita, Algebraic structure of countably compact Abelian groups of size continuum from selective ultrafilters, submitted.

[8] _ A countably compact group topology on Abelian almost torsion-free groups from selective ultrafilters, to appear in Houston J. Math.

[9] _ A group topology on the free Abelian group of cardinality $\mathfrak{c}$ that makes its square countably compact, Fund. Math. 212 (2011), 235-260. 
[10] T. Bröcker and T. tom Dieck, Representations of compact Lie groups, Springer, 1985.

[11] I. Castro-Pereira and A. H. Tomita, Abelian torsion groups with a countably compact group topology, Topology Appl. 157 (2010), 44-52.

[12] W. W. Comfort, Problems on topological groups and other homogeneous spaces, Open problems in topology (J. van Mill and G. M. Reed, eds.), North-Holland, 1990, pp. 313-347.

[13] W. W. Comfort, K. H. Hofmann, and D. Remus, Topological groups and semigroups, Recent progress in general topology (M. Husek and J. van Mill, eds.), North-Holland, 1992, pp. 57144.

[14] D. Dikranjan and D. Shakhmatov, Algebraic structure of pseudocompact groups, Memoirs Amer. Math. Soc. 133 (1998).

[15] _ Forcing hereditarily separable compact-like group topologies on Abelian groups, Topology Appl. 151 (2005), 2-54.

[16] Selected topics from the structure theory of topological groups, Open problems in topology II (E. Pearl, ed.), Elsevier, 2007, pp. 389-406.

[17] D. Dikranjan and M. G. Tkachenko, Algebraic structure of small countably compact Abelian groups, Forum Math. 15 (2003), 811-837.

[18] E. K. van Douwen, The product of two countably compact topological groups, Trans. Amer. Math. Soc. 262 (1980), 417-427.

[19] R. Engelking, General topology, Heldermann, 1989.

[20] S. Garcia-Ferreira, A. H. Tomita, and S. Watson, Countably compact groups from a selective ultrafilter, Proc. Amer. Math. Soc. 133 (2005), 937-943.

[21] D. L. Grant, Sequentially compact cancellative topological semigroups: some progress on the Wallace problem, Ann. N. Y. Acad. Sci. 704 (1993), 150-154.

[22] A. Hajnal and L. Juhász, A separable normal topological group need not be Lindelöf, Gen. Topology Appl. 6 (1976), 199-205.

[23] P. R. Halmos, Comment on the real line, Bull. Amer. Math. Soc. 50 (1944), 877-878. 
[24] D. K. Harrison, Infinite Abelian groups and homological methods, Ann. Math. 69 (1959), 366-391.

[25] A. Hulanicki, Algebraic structure of compact Abelian groups, Bull. Acad. Polon. Sci. Sér. Sci. Math. Astr. Phys. 6 (1958), 71-73.

[26] T. Jech, Set theory, Springer, 2003.

[27] I. Kaplansky, Infinite Abelian groups, University of Michigan Press, 1954.

[28] K. Kunen, Some points in $\beta \mathbb{N}$, Proc. Cambridge Philos. Soc. 80 (1976), 385-398.

[29] _ Set theory: an introduction to independence proofs, North Holland, 1983.

[30] R. E. Madariaga-Garcia and A. H. Tomita, Countably compact topological group topologies on free Abelian groups from selective ultrafilters, Topology Appl. 154 (2007), 1470-1480.

[31] K. Numakura, On bicompact semigroups, Math. J. Okayama Univ. 1 (1952), 99-108.

[32] D. Robbie and S. Svetlichny, An answer to A. D. Wallace's question about countably compact cancellative semigroups, Proc. Amer. Math. Soc. 124 (1996), 325-330.

[33] D. J. S. Robinson, A course in the theory of groups, Springer, 1995.

[34] C. T. Scarborough and A. H. Stone, Products of nearly compact spaces, Trans. Amer. Math. Soc. 124 (1966), 131-147.

[35] M. G. Tkachenko, Countably compact and pseudocompact topologies on free Abelian groups, Soviet Math. (Izv. VUZ) 34 (1990), 79-86.

[36] _ Topological groups: between compactness and $\aleph_{0}$-boundedness, Recent progress in general topology II (J. van Mill M. Husek, ed.), Elsevier, 2002, pp. 515-543.

[37] M. G. Tkachenko and I. Yaschenko, Independent group topologies on Abelian groups, Topology Appl. 122 (2002), 425-451.

[38] A. H. Tomita, The existence of initially $\omega_{1}$-compact group topologies on free Abelian groups is independent of ZFC, Comment. Math. Univ. Carolinae 39 (1998), 401-413.

[39] - On the number of countably compact group topologies on a free Abelian group, Topology Appl. 98 (1999), 345-353. 
[40] _ A solution to Comfort's question on the countable compactness of powers of a topological group, Fund. Math. 186 (2005), 1-24.

[41] A. H. Tomita and S. Watson, Ultraproducts, p-limits and antichains on the Comfort group order, Topology Appl. 143 (2004), 147-157.

[42] J. E. Vaughan, Products of perfectly normal sequentially compact spaces, J. London Math. Soc. 14 (1976), 517-520.

[43] _ Small uncountable cardinals and topology, Open problems in topology (J. van Mill and G. M. Reed, eds.), North-Holland, 1990, pp. 195-218.

[44] _ The Scarborough-Stone problem, Open problems in topology II (E. Pearl, ed.), Elsevier, 2007, pp. 249-256.

[45] A. D. Wallace, The structure of topological semigroups, Bull. Amer. Math. Soc. 61 (1955), 95-112. 
Índice Remissivo

$\Delta$-sistema, 11

$\epsilon$-denso, 9

anticadeia, 165

aplicação

cofinal, 11

axioma de Martin, 165

para ordens parciais enumeráveis, 165

base

para um filtro, 12

caráter

de um ponto, 9

cardinal

regular, 11

singular, 11

cofinalidade, 11

denso, 165

espaço

enumeravelmente compacto, 8

pseudocompacto, 8

filtro, 12 genérico, 165

gerado, 12

grupo

de não torção, 6

de torção, 6

de torção limitada, 6

divisível, 7

quase livre de torção, 6

quase-cíclico, 7

topológico, 10

grupos topológicos

isomorfos, 10

incompatíveis, 165

isomorfismo

de grupos topológicos, 10

periódico, 156

peso, 9

ponto

de acumulação, 8

produto diagonal, 28

raiz 
n-ésima, 7

n-ésima da unidade, 7

do $\Delta$-sistema, 11

semigrupo, 10

topológico, 10

subconjunto

independente, 6

subgrupo

de torção, 6

suporte, 6

ultrafiltro, 12

livre, 12

principal, 12 